\title{
The Nature and Structure of Temperament in Early and Middle Childhood: A Multi-Method Approach
}

Yuliya Kotelnikova, The University of Western Ontario

Supervisor: Dr. Elizabeth P. Hayden, The University of Western Ontario

Joint Supervisor: Dr. Tony Vernon, The University of Western Ontario

A thesis submitted in partial fulfillment of the requirements for the Doctor of Philosophy degree in Psychology

(C) Yuliya Kotelnikova 2016

Follow this and additional works at: https://ir.lib.uwo.ca/etd

Part of the Child Psychology Commons, Clinical Psychology Commons, Developmental Psychology Commons, Personality and Social Contexts Commons, and the Quantitative Psychology Commons

\section{Recommended Citation}

Kotelnikova, Yuliya, "The Nature and Structure of Temperament in Early and Middle Childhood: A MultiMethod Approach" (2016). Electronic Thesis and Dissertation Repository. 3819.

https://ir.lib.uwo.ca/etd/3819

This Dissertation/Thesis is brought to you for free and open access by Scholarship@Western. It has been accepted for inclusion in Electronic Thesis and Dissertation Repository by an authorized administrator of Scholarship@Western. For more information, please contact wlswadmin@uwo.ca. 


\begin{abstract}
Structural work on the nature of child temperament has lagged far behind parallel research with adults. Further, the structure of extant parent-report questionnaires has been examined only at the higher-order level, using small samples of children. This dissertation addresses this important gap in three studies, examining the lower- and higher-order structure of two widely used parent-reports of child temperament (Studies 1 and 2) and an observational battery of temperament (Study 3). The first study examined the lower- and higher-order structure of the Children's Behavior Questionnaire (CBQ; Rothbart, Ahadi, Hershey, \& Fisher, 2001) in a large community sample of children at ages $3(N=944)$ and 5/6 $(N=853)$. The second study extended this work by examining the structure of the Temperament in Middle Childhood Questionnaire (TMCQ; Simonds \& Rothbart, 2004) in a large $(N=654)$ sample of 9-year-olds. Fewer than half of the lower-order structures resembled original CBQ and TMCQ scales. Higher-order EFAs indicated that a four-factor structure consisting of Sensation Seeking, Disinhibition/Anger, Low Negative Affect (NA)/Soothability, and Smiling/Approach was the best fit for preschoolers, and that a three-factor structure consisting of Impulsivity/Anger, NA, and Social Dominance best fit the TMCQ data. The higher-order models obtained were only modestly similar to the models proposed by their developers. In the third study, we extended the scarce extant literature on the structure of observed temperament. Using exploratory structural equation modeling, we derived a four-factor model (Positive Affect/Activity, Impulsivity/Anger, Surgency, and Dysphoria) of preschool-aged temperament. These factors showed meaningful concurrent associations with children's internalizing and externalizing symptoms, providing further support for the use of observational measures. Findings from these studies contribute to the development of a unified taxonomy of child temperament/personality.
\end{abstract}


Keywords: child temperament, early and middle childhood, parent reports, observational methods, exploratory and confirmatory factor analysis, measurement invariance, exploratory structural equation modeling, internalizing and externalizing symptoms 


\section{Co-authorship Statement}

Several co-authors are listed on the three manuscripts that comprise this doctoral thesis. In particular, Dr. Daniel N. Klein (Department of Psychology, Stony Brook University, Long Island, NY) is listed as a co-author on all three manuscripts. Both Dr. Klein, who is a principal investigator on all of the projects that yielded the data collected in Long Island, NY, and my research supervisor Dr. Elizabeth P. Hayden have provided me with invaluable help in formulating my hypotheses, interpreting results, and linking the findings to extant literature on child temperament. Dr. Thomas M. Olino (Department of Psychology, Temple University) is listed as a co-author on all three papers; he has provided me with continual support with data analyses for all three projects. Dr. Katie R. Kryski is listed as a co-author on the first and third manuscripts and Sarah V.M. Mackrell is listed as a co-author on the second paper. They have been actively involved in data collection that took place at Western University, London, ON. My own involvement in all three projects is comprised of the following: data collection, video coding, preparation of data for analyses, literature review and conceptualization of hypotheses, acquisition of the necessary statistical skills, data analyses, interpretation of the findings within the context of extant literature on child temperament and psychopathology, and working on numerous drafts of all papers with support from Dr. Hayden, Dr. Klein, and Dr. Olino. 


\section{Acknowledgements}

There are many people to thank for their support throughout this six-year journey. First and foremost, I would like to express my utmost gratitude to my supervisors, Dr. Elizabeth P. Hayden and Dr. Tony Vernon, for their unwavering support and outstanding mentorship throughout this process. It was a privilege to work with both of these outstanding researchers.

With assistance from Dr. Hayden, I also had an amazing opportunity to work with Dr. Daniel N. Klein and Dr. Thomas M. Olino, who have been terrific mentors to me over the past six years.

To my Ph.D. supervisory and examination committee members, Dr. Jim Neufeld, Dr. Paul Tremblay, and Dr. Len Simms, your statistical expertise has been invaluable for my thesis. In particular, Dr. Tremblay was available for ongoing consultation. I would also like to thank Dr. Adam Cohen and Dr. Alan Leschied for their time, insight, and expertise in conceptualizing my work.

I would also like to thank my fellow graduate students and research coordinators who helped with collecting all these amazing data over several years making this thesis possible: Katie R. Kryski, Heather J. Smith, Rachel Nott, Jasmine Desjardins, and Emily Johnson. Thank you all very much for the outstanding work you have done!

I would also like to express my gratitude to the Social Sciences and Humanities Research Council, Ontario Graduate Scholarship Program, Children's Health Research Institute (London, ON), and Western University for funding my masters and doctoral research over the past six years.

To my parents, my aunt, and my grandfather, thank you for inspiring to become a clinical scientist! To my close friends, Sarah Mackrell, Francois Botha, Karen Zhang, and Felicia Pavlovic, I could not have done this without you! 


\section{Table of Contents}

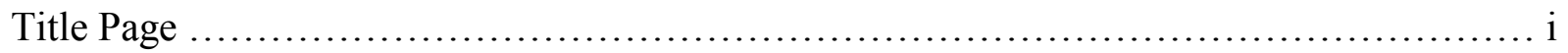

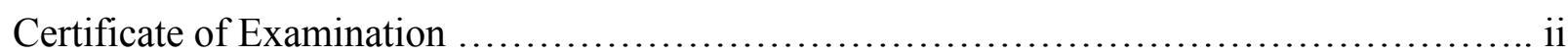

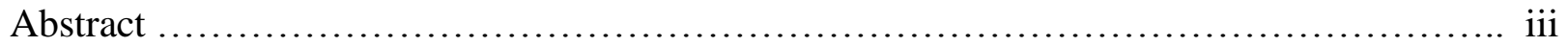

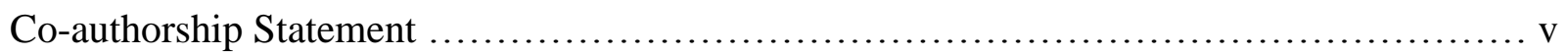

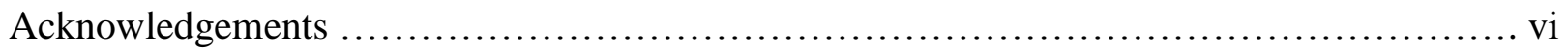

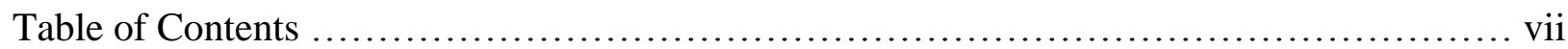

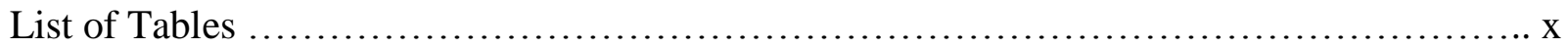

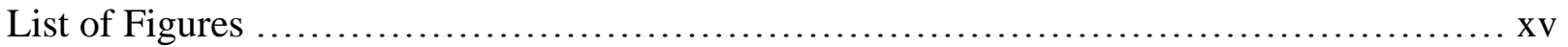

List of Appendices ..........................................................

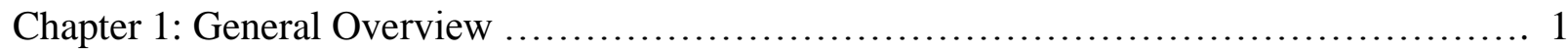

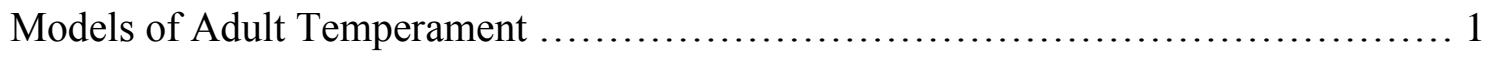

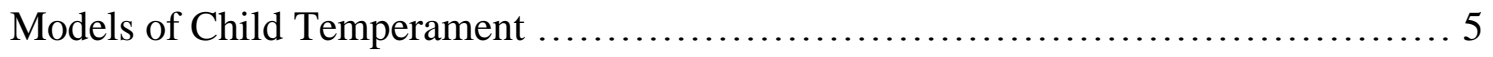

Methods Used to Assess Child Temperament ............................... 9

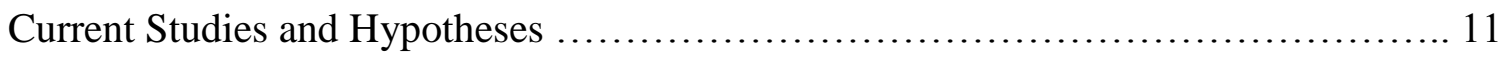

References ................................................................ 14

Chapter 2: Higher- and Lower-Order Structure of Children's Behavior Questionnaire in Early

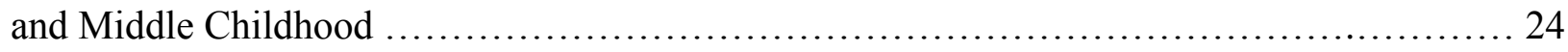

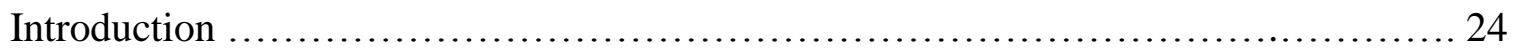

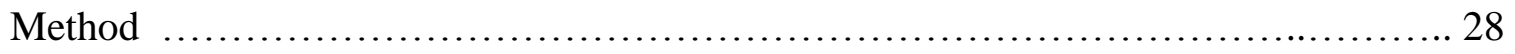

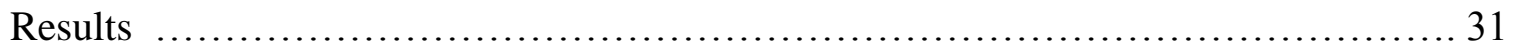

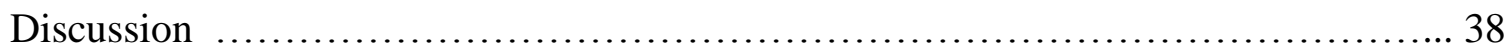

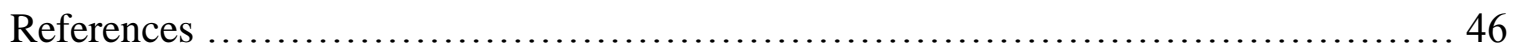


Tables

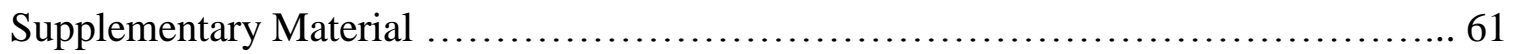

Chapter 3. Higher- and Lower-Oder Structure of Temperament in Middle Childhood

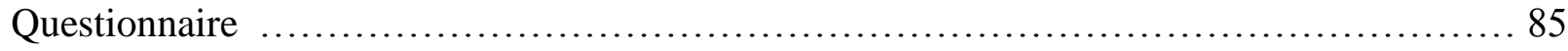

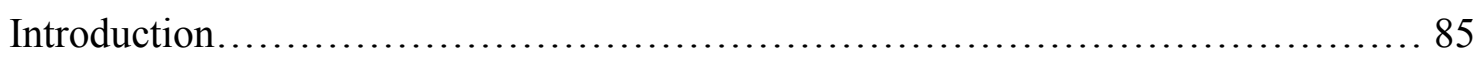

Method.................................................................. 89

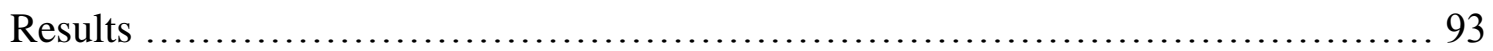

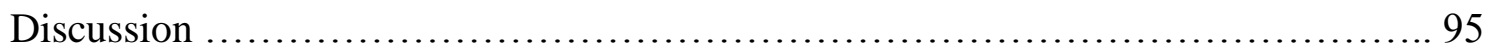

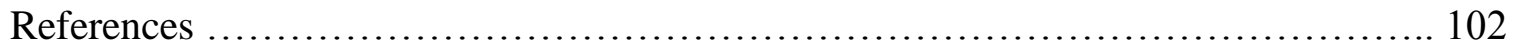

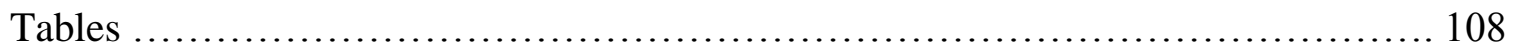

Chapter 4. The Structure of Observed Temperament in Preschoolers....................... 115

Introduction........................................................... 115

Method.................................................................. 123

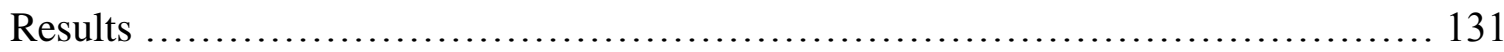

Discussion ......................................................... 133

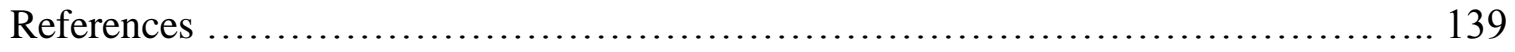

Tables and Figures................................................ 153

Supplementary Material ............................................... 158

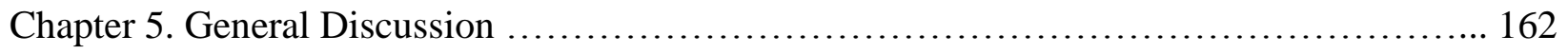

General Strengths and Weaknesses of the Three Studies and Future Directions .............. 169

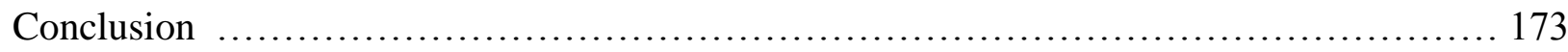

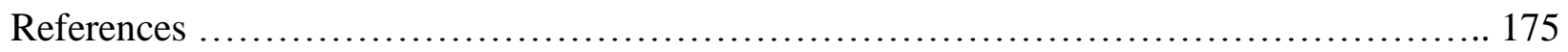

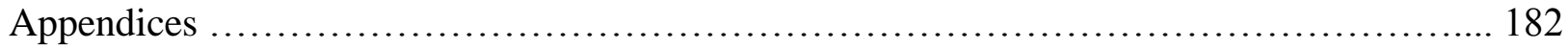


Curriculum Vitae . 


\section{List of Tables}

General Overview Table 1: Common Dimensions of Child Temperament $\ldots \ldots \ldots \ldots \ldots \ldots \ldots 5$

Higher- and Lower-Order Structure of Children's Behavior Questionnaire in Early and Middle Childhood Table 1: Sample Descriptive Statistics .................................... 53

Higher- and Lower-Order Structure of Children's Behavior Questionnaire in Early and Middle

Childhood Table 2: Descriptive Statistics for the 15 Original CBQ Scales

Higher- and Lower-Order Structure of Children's Behavior Questionnaire in Early and Middle Childhood Table 3: Exploratory Factor Analysis of the CBQ Items at Age $3 \ldots \ldots \ldots \ldots \ldots \ldots 5$

Higher- and Lower-Order Structure of Children's Behavior Questionnaire in Early and Middle Childhood Table 4: Exploratory Factor Analyses of the CBQ Items at Age 5/6

Higher- and Lower-Order Structure of Children's Behavior Questionnaire in Early and Middle Childhood Table 5: Higher-Order Exploratory Factor Analyses of the CBQ Lower-Order Scales

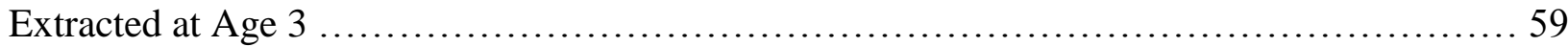

Higher- and Lower-Order Structure of Children's Behavior Questionnaire in Early and Middle Childhood Table 6: Higher-Order Exploratory Factor Analysis of the CBQ Lower-Order Scales

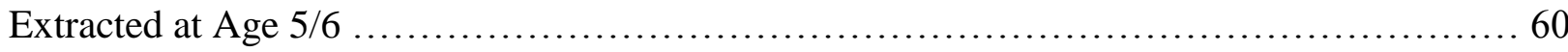


Higher- and Lower-Order Structure of Children's Behavior Questionnaire in Early and Middle Childhood Table A: Lower-Order CFA Results for Original CBQ Scales at Age 3 61

Higher- and Lower-Order Structure of Children's Behavior Questionnaire in Early and Middle Childhood Table B: Lower-Order CFA Results for the Original CBQ Scales at Age 5/6 ..... 67

Higher- and Lower-Order Structure of Children's Behavior Questionnaire in Early and Middle Childhood Table C: CFA solution for the Three Higher-Order CBQ Factors reported by Rothbart

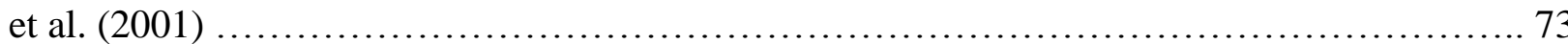

Higher- and Lower-Order Structure of Children's Behavior Questionnaire in Early and Middle Childhood Table D: Items Excluded from Higher-Order EFA Analyses at Age 3 Due to Low

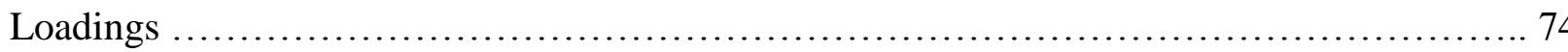

Higher- and Lower-Order Structure of Children's Behavior Questionnaire in Early and Middle Childhood Table E: Items Excluded from Higher-Order EFA Analyses at Age 5/6 Due to Low Loadings

Higher- and Lower-Order Structure of Children's Behavior Questionnaire in Early and Middle Childhood Table F: Higher-Order Exploratory Factor Analysis of the CBQ Lower-Order Scales Extracted at Age 3: Three-Factor Solution 80

Higher- and Lower-Order Structure of Children's Behavior Questionnaire in Early and Middle 
Childhood Table G: Tests of Structural Invariance across the Two Samples (ON and NY) ... 81

Higher- and Lower-Order Structure of Children's Behavior Questionnaire in Early and Middle Childhood Table H: Higher-Order Exploratory Factor Analysis of the CBQ Lower-Order Scales Extracted at Age 5/6: Three-Factor Solution ..................................... 82

Higher- and Lower-Order Structure of Children's Behavior Questionnaire in Early and Middle Childhood Table I: Higher-Order Exploratory Factor Analysis of the CBQ Lower-Order Scales Extracted at Age 5/6: Three-Factor Solutions in NY and ON Samples 83

Higher- and Lower-Order Structure of Children's Behavior Questionnaire in Early and Middle Childhood Table J: Higher-Order Exploratory Factor Analysis of the CBQ Lower-Order Scales Extracted at Age 5/6: Four-Factor Solutions in NY and ON Samples 84

Higher- and Lower-Oder Structure of Temperament in Middle Childhood Questionnaire Table 1:

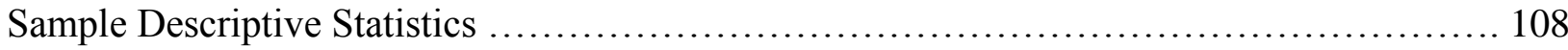

Higher- and Lower-Oder Structure of Temperament in Middle Childhood Questionnaire Table 2: Descriptive Statistics for the 17 Original TCMQ Scales 109

Higher- and Lower-Oder Structure of Temperament in Middle Childhood Questionnaire Table 3: Exploratory Factor Analysis for the TMCQ Items 110 
Higher- and Lower-Oder Structure of Temperament in Middle Childhood Questionnaire Table 4: Bivariate Associations between the 17 Original TMCQ scales and the 13 New Lower-Order

Factors 112

Higher- and Lower-Oder Structure of Temperament in Middle Childhood Questionnaire Table 5: Higher-Order Exploratory Factor Analyses of the TMCQ Lower-Order Scales 113

Higher- and Lower-Oder Structure of Temperament in Middle Childhood Questionnaire Table 6: Tests of Structural Invariance across the Two Samples (ON and NY): Three-Factor Model.... 114

The Structure of Observed Temperament in Preschoolers Table 1: Bivariate Associations Between Major Study Variables 153

The Structure of Observed Temperament in Preschoolers Table 2: Structure of Temperament in Preschoolers: ESEM Results 154

The Structure of Observed Temperament in Preschoolers Table 3: Bivariate Associations Between ESEM Factors 155

The Structure of Observed Temperament in Preschoolers Table 4: Bivariate Associations Between Temperament Factors and Concurrent Parent-Reported Symptoms of Psychopathology 
General Discussion Table 1: Higher-order structure of child temperament based on child age and method of assessment from Kotelnikova, Olino, Klein, Kryski, \& Hayden (2016), Kotelnikova, Olino, Klein, Mackrell, \& Hayden (2016), and Kotelnikova, Kryski, Olino, Klein, \& Hayden (2016) 163 


\section{List of Figures}

General Overview Figure 1: Hierarchical Structure of Adult Temperament and Personality ... 4

The Structure of Observed Temperament in Preschoolers Figure 1: Structure of Temperament in

Preschoolers................................................................ 157

The Structure of Observed Temperament in Preschoolers Figure A: CFA in NY Sample..... 161 


\section{List of Appendices}

Appendix A: Ethics Approval for the Data Used in "Higher- and Lower-Order Factor Analyses of the Children's Behavior Questionnaire in Early and Middle Childhood" and "The Structure of Observed Temperament in Preschoolers" ON Sample.................................... 182

Appendix B: Ethics Approval for the Data Used in "Higher- and Lower-Order Factor Analyses of the Temperament in Middle Childhood Questionnaire" ON sample...................... 183

Appendix C: Ethics Approval for the Data Used in "Higher- and Lower-Order Factor Analyses of the Children's Behavior Questionnaire in Early and Middle Childhood", "Higher- and LowerOrder Factor Analyses of the Temperament in Middle Childhood Questionnaire", and "The Structure of Observed Temperament in Preschoolers" NY Sample................................. 184 


\section{General Overview}

Temperament is an ancient concept dating back to at least the $5^{\text {th }}$ century B.C., at which point interest in linking temperament to physical and mental health was in its nascent stages (Clark \& Watson, 2008; Digman, 1994). Despite the passage of time, aspects of ancient formulations of temperament are evident in modern conceptualizations, in that biological factors are thought to underlie observable manifestations of temperament, and that individual differences in emotion expression are seen as key features (Clark \& Watson, 2008). Biological influences and emotion are also highly relevant to personality constructs (Allport, 1937), suggesting that there is a significant conceptual overlap between the domains of temperament and personality (Digman, 1994; Watson \& Clark, 1993). Indeed, both show similar heritability estimates (e.g., Jang, McCrae, Angleitner, Riemann, \& Livesley, 1998; Saudino, 2005) and stability across time (De Pauw \& Mervielde, 2010). In light of this overlap, there is a general consensus in the field that temperament and personality are not as distinct as once thought, and that the two capture the same essential phenomena (Clark \& Watson, 2008; Shiner \& DeYoung, 2013). As a result, this doctoral thesis builds on a united conceptualization of personality and temperament, integrating the two literatures as necessary.

\section{Models of Adult Temperament/Personality}

Significant research has accumulated on both the nature and structure of adult personality and temperament, showing that contemporary models that differ in terms of the number of core traits can be arranged hierarchically (DeYoung, 2006; Digman, 1997; Markon, Krueger, \& Watson, 2005; McCrae, Yamagata, Jang, Riemann, Ando, Ono et al., 2008; Zuckerman, Kuhlman, \& Camac, 1988; see Figure 1). At the highest level of the hierarchy, adult temperament has been reduced to two superfactors: alpha is comprised of negative emotionality 
(NE) and disinhibition (versus constraint), and beta is comprised of positive emotionality (PE; Markon et al., 2005). At the next level, the model is broadly consistent with three-factor temperament models (e.g., Eysenck, 1967; Tellegen, 1985; Watson \& Clark, 1993) that identify dimensions capturing individual differences in NE, PE, and disinhibition (i.e., impulsivity). At the subsequent levels of the hierarchy, disinhibition is parsed into the relatively narrow traits of disagreeableness and low conscientiousness, and PE separates into two traits of extraversion and openness to experience, thus yielding the full Five Factor Model (FFM; Digman, 1990; McCrae \& Costa, 1997). Some researchers (e.g., Livesley, Jang, \& Vernon, 1998; Nitschke, Heller, Imig, McDonald, \& Miller, 2001; Watson, Wu, \& Cutshall, 2004) have proposed even more finely differentiated traits, although the utility of such a level of differentiation remains unclear (Clark, 2005).

Strong evidence supporting the stability of higher-order and midlevel temperament/personality traits has accumulated during the past 20 years, supporting the construct validity of the traits described above (Caspi, 2000; Eisenberg, Guthrie, Murphy, Shepard, Cumberland, \& Carlo, 1999; Eisenberg,Valiente, Spinrad, Cumberland, Liew, Reiser et al., 2009; Laursen, Pulkkinen, \& Adams, 2002; Pesonen, Raikkonen, Keskivaara, \& KeltikangasJarvinen, 2003; Shiner, Masten, \& Tellegen, 2002). For example, the large-scale Australian "Dunedin" study showed that children identified as undercontrolled at age 3 were impulsive, unreliable, and antisocial as adults, whereas those classified as inhibited were more likely to experience depressive symptoms and have scarce social supports in adulthood (Caspi, 2000). Additionally, Shiner et al. (2002) found evidence for developmental continuity between affective and regulatory traits assessed at age 8 and the same traits in young adulthood. Similar findings were obtained for compliance, self-control, and aggression (Laursen et al., 2002). Finally, 
Roberts and DelVecchio (2000) also provided some evidence that stability of temperament and personality traits increases with age by conducting a meta-analysis of 152 longitudinal studies. Thus, findings indicate that temperament/personality traits show a fairly high level of stability across the lifespan, and that trait rank-order consistency may increase over time, reaching its peak during the ages 50 to 59 .

The stability of temperament traits raises the question of their predictive validity for outcomes of interest. As such, a key goal of temperament/personality research is to predict important developmental outcomes; indeed, models that lack the capacity to do so are limited in value. Given the conceptual overlap between traits and symptoms of psychopathology (i.e., temperament traits have been conceptualized by some as forms frustes suggesting that aspects of temperament may represent an incomplete form of disorder themselves; Klein, Durbin, \& Shankman, 2009), the predictive validity of personality for mental disorders has been examined extensively. In particular, the highly influential tripartite model of anxiety and depression (Clark \& Watson, 1999) posits that higher levels of NE are etiologically relevant to both anxiety and depression, whereas lower levels of PE are related to depression only. Later, this framework was expanded to include other disorders due to evidence that aspects of low PE (e.g., anhedonia) are also correlated with social phobia and schizophrenia/schizotypy (Clark, 2005; Watson, Gamez, \& Simms, 2005; Watson, Kotov, \& Gamez, 2006). Similarly, the role of NE was expanded in light of findings showing strongest and most consistent associations observed between NE and disorders characterized by subjective, chronic distress and dysphoria (e.g., depression and GAD) than with types of dysfunction characterized by more acute forms of distress (e.g., panic disorder, specific phobias; Watson et al., 2005).

Although extensive research on the structure of adult temperament/personality and the 
validity of the three- and five-factor models has accumulated over the past 20 years, structural models of youth temperament have a much shorter history, and there is no consensus on the number and nature of primary dimensions of temperament in childhood (De Pauw \& Mervielde, 2010). Similarly, the evidence for the rank-order consistency of temperament traits across infancy and childhood ranges from relatively low to moderate (e.g., from 0 to .65; Kochanska, Murray, \& Coy, 1997; Matheny, 1989; McDevitt, 1986), suggesting that childhood represents a developmental period characterized by the transformation of these traits into adult personality through acquisition of important developmental skills (e.g., perspective taking, development of self-concept; Roberts \& DelVecchio, 2000). According to Shiner (1998), as children age, temperament traits become more differentiated and hierarchically integrated, suggesting important changes in their overarching structure. However, the exact nature of the process of transformation of child temperament traits into adult personality remains unclear (Roberts \& DelVecchio, 2000; Shiner \& DeYoung, 2013).

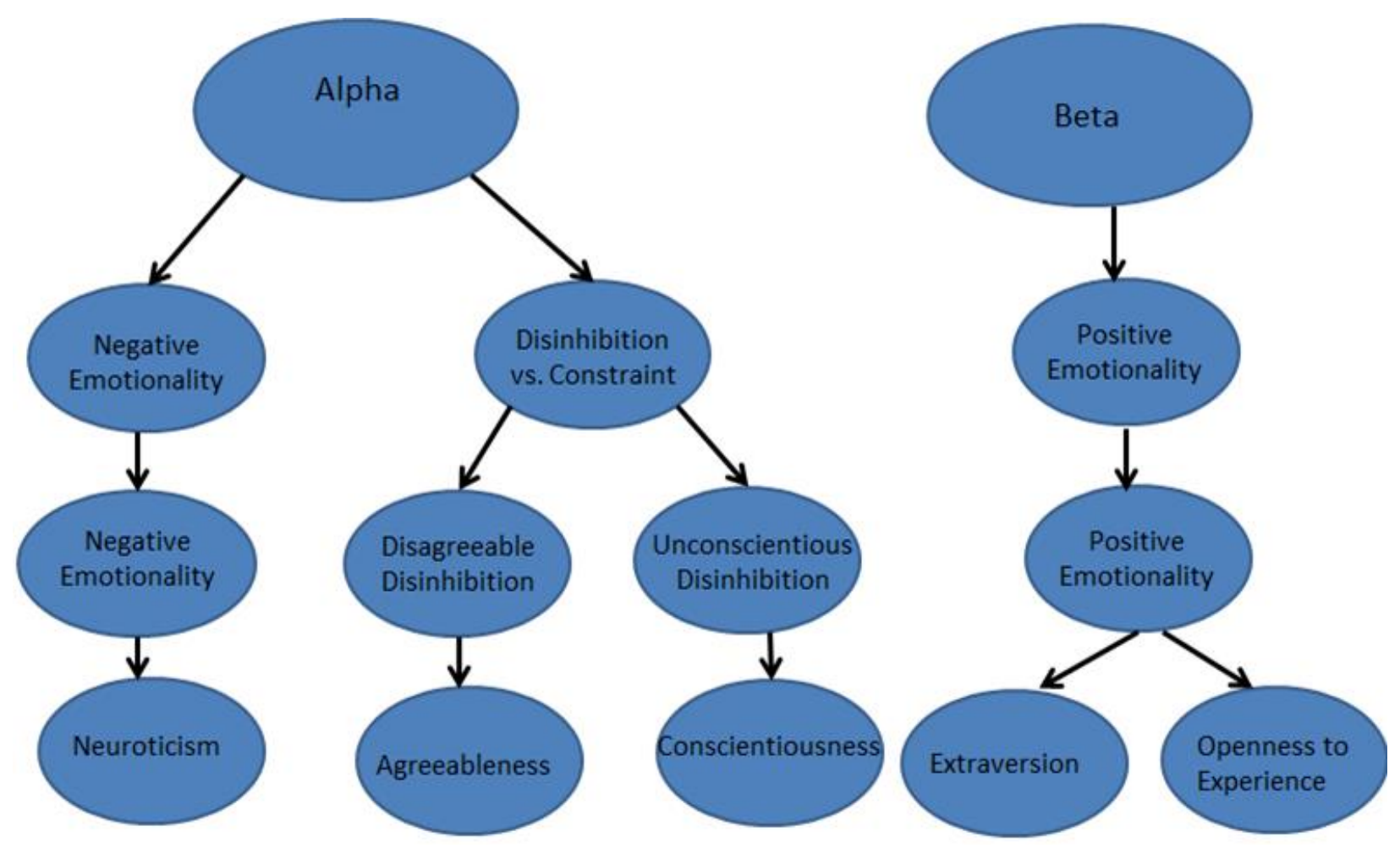


Figure 1. Hierarchical structure of adult temperament and personality adapted from Markon,

Krueger, and Watson (2005).

\section{Models of Child Temperament}

Although generally lagging behind the work on adult temperament/personality structure, several influential models of child temperament have been articulated by developmental psychologists; the most popular of these are summarized in Table 1 with an emphasis on their common dimensions (Buss \& Plomin, 1984; Dyson, Olino, Durbin, Goldsmith, \& Klein, 2012; Rothbart, 1981; Shiner \& Caspi, 2012; Thomas \& Chess, 1977). While these models have made important contributions to our understanding of child temperament, none except for the fivefactor model were developed using what would be considered optimal methodologies defined by contemporary standards. More specifically, older models have relied on relatively small samples and lacked sophistication in their statistical analyses such as the absence of use of structural equation modeling which is common for studies of adult personality (McCrae, Costa, Del Pilar, Rolland, \& Parker, 1998).

\begin{tabular}{l|l|l|l|l|l|l}
\hline $\begin{array}{l}\text { Common } \\
\text { Dimensions }\end{array}$ & $\begin{array}{l}\text { Thomas \& } \\
\text { Chess }\end{array}$ & $\begin{array}{l}\text { Buss \& } \\
\text { Plomin }\end{array}$ & $\begin{array}{l}\text { Rothbart et } \\
\text { al. }\end{array}$ & DePauw et al. & Caspi \& Shiner & Dyson et al. \\
\hline Extraversion & $\begin{array}{l}\text { Social } \\
\text { Inhibition }\end{array}$ & Sociability & $\begin{array}{l}\text { Extraversion } \\
\text { /Surgency }\end{array}$ & Sociability & $\begin{array}{l}\text { Extraversion/ } \\
\text { Positive } \\
\text { Emotionality }\end{array}$ & $\begin{array}{l}\text { PA/Interest; } \\
\text { Sociability }\end{array}$ \\
\hline Activity & Activity Level & Activity & & Activity & & \\
\hline Neuroticism & $\begin{array}{l}\text { Negative } \\
\text { Emotionality }\end{array}$ & Emotionality & $\begin{array}{l}\text { Negative } \\
\text { Affectivity }\end{array}$ & Emotionality & $\begin{array}{l}\text { Neuroticism/ } \\
\text { Negative } \\
\text { Emotionality }\end{array}$ & $\begin{array}{l}\text { Dysphoria; } \\
\text { Fear/BI }\end{array}$ \\
\hline $\begin{array}{l}\text { Conscientious } \\
\text { ness }\end{array}$ & $\begin{array}{l}\text { Task } \\
\text { Persistence }\end{array}$ & & $\begin{array}{l}\text { Effortful } \\
\text { Control }\end{array}$ & Persistence & $\begin{array}{l}\text { Conscientiousness } \\
\text { /Constraint }\end{array}$ & $\begin{array}{l}\text { Constraint } \\
\text { vs. } \\
\text { Impulsivity }\end{array}$ \\
\hline $\begin{array}{l}\text { Agreeableness } \\
\text { /Adaptability }\end{array}$ & $\begin{array}{l}\text { Agreeabless } \\
\text { Expenness to }\end{array}$ & & $\begin{array}{l}\text { Sociability/ } \\
\text { Affiliation }\end{array}$ & Disagreeableness & Agreeableness & \\
\hline
\end{tabular}

Table 1. Common dimensions of child temperament: this table was adapted from De Pauw, S. S. W., Mervielde, I., \& Van Leeuwen, K. G. (2009) and Dyson et al., 2012. 
Some of the earliest research on the structure of child temperament was conducted by Thomas and Chess (1977) who defined temperament as the "stylistic component" or the "how" of behavior. They hypothesized that, despite similar motivations, children are likely to differ with regards to how they perform a certain behavior based on differences in their temperament traits such as emotional expression, persistence, distractibility, etc. (Goldsmith, Buss, Plomin, Rothbart, Thomas, Chess et al., 1987). It was noted that temperament traits are biologically determined yet expressed primarily as a response to opportunities, expectations, or demands in the environment (Goldsmith et al., 1987). Based on their analysis of parent reports of infant behavior, Thomas and Chess (1977) proposed that nine bipolar dimensions capture the bulk of meaningful variance in child temperament: activity, approach/withdrawal, adaptability, mood, threshold of responsiveness/sensitivity, intensity of reaction, distractibility, rhythmicity/regularity, and attention span/task persistence. However, subsequent factor analytic studies (Martin, Wisenbaker, \& Huttunen, 1994; Presley \& Martin, 1994) did not support the existence of all nine dimensions; instead, the following five have emerged: negative emotionality, social inhibition, agreeableness/adaptability, task persistence, and activity level (independence of this last factor remains questionable; Presley \& Martin, 1994).

In contrast to the fairly complex, multifaceted model proposed by Thomas and Chess, Buss and Plomin (1984) posited that child temperament could be captured by a more parsimonious model consisting of emotionality, activity, and sociability (EAS model). The EAS model was based on the evidence that its core traits are present in non-human animals, as well as infants, children, and adults, and that emotionality, activity, and sociability were also deemed to be among the most heritable traits (Buss \& Plomin, 1984). The first two factors of the EAS model (emotionality and activity) show some overlap with the dimensions proposed by Thomas 
and Chess (1977; see Table 1) instantiated in the presence of such traits as activity, mood, and intensity of reaction. Thus, although the two models differ in terms of the number of core traits, there is some overlap in their content.

Rothbart (1981) further refined conceptualizations of child temperament that existed at the time by including two levels of analysis (i.e., lower-order traits are subsumed under higherorder factors). She conceptualized child temperament as reflecting individual differences in emotional reactivity (i.e., excitability in response to changes in internal and external environment) and self-regulation (i.e., the ability to modulate reactive processes; Rothbart, 1981). This conceptualization has been instantiated in several caregiver-report measures that span children's development, including the Children's Behavior Questionnaire (CBQ; Rothbart, Ahadi, Hershey, \& Fisher, 2001; ages 3-6), and the Temperament in Middle Childhood Questionnaire (TMCQ; Simonds, 2006; Simonds \& Rothbart, 2004; ages 7-10). Each of these measures has lower-order scales that were developed via a top-down, theory-based approach, relying heavily on the measures developed earlier by Thomas and Chess (1977). Thus, both the CBQ and the TMCQ include numerous lower-order scales that bear significant resemblance to the nine bipolar dimensions developed by Thomas and Chess (1977; e.g., activity, approach/withdrawal, threshold of responsiveness/sensitivity, intensity of reaction, and attention span/task persistence). Higher-order factor-analytic studies conducted on the CBQ scales have yielded three broad dimensions that generally support Rothbart's model of temperament in young children (see Table 1): Extraversion/Surgency, Negative Affectivity (NA; both of which are related to the emotionality factor from the EAS model by Buss \& Plomin, 1984) and Effortful Control (EC; Ahadi, Rothbart, \& Ye, 1993; Rothbart et al., 2001). Further, Simonds and Rothbart (2004) reported additional, albeit inconsistent, evidence for a possible fourth 
higher-order factor that emerges in older children, Sociability/Affiliation (this factor also bears similarity to the sociability factor from the EAS model by Buss \& Plomin, 1984). Overall, research conducted by Rothbart and her colleagues (Rothbart, 1981; Rothbart et al., 2001; Simonds \& Rothbart, 2004) indicates that the structure of child temperament resembles several prominent three-factor models of adult personality that include reactive (i.e., Extraversion/Surgency and NA) and regulatory (i.e., EC) components (e.g., Eysenck, 1967; Tellegen, 1985; Watson \& Clark, 1993). However, extant literature on competing models of child temperament that were developed via a purely top-down, theory-based approach has been inconclusive and, as a result, more empirical studies using larger samples and more sophisticated statistical approaches are required to test these models.

Finally, given the extensive support for the FFM as a comprehensive empirically-based higher-order taxonomy of personality traits in adults across genders, cultures, and methods (e.g., Digman 1990; Goldberg, 1993; McCrae \& Costa, 1997), developmental psychologists have begun to examine the validity this model for understanding personality in children and adolescents (Caspi \& Shiner, 2006; Shiner \& Caspi, 2012; see Table 1). Several researchers have provided evidence for the five-factor structure from parent-report measures in older children and adolescents (e.g., Barbaranelli, Caprara, Rabasca, \& Pastorelli, 2003; John, Caspi, Robins, Moffit, \& Stouthamer-Loeber, 1994), teacher-reports (e.g., Barbaranelli et al., 2003; Digman \& Shmelyov, 1996; Goldberg, 2001; Graziano \& Ward, 1992; Mervielde, Buyst, \& De Fruyt, 1995), and self-reports (e.g., Barbaranelli et al., 2003; De Fruyt, Mervielde, Hoekstra, \& Roland, 2000; Markey, Markey, Tinsley, \& Erikson, 2002). However, despite several attempts at recovering the same structure in younger samples (e.g., Abe, 2005; Abe \& Izard, 1999; Halverson, Havill, Deal, Baker,Victor, Pavlopoulous et al., 2003; Tackett, Slobodskaya, Mar, 
Deal, Halverson, Baker et al., 2012), the evidence for a five-factor model of personality in childhood remains unclear (e.g., De Pauw \& Mervielde, 2010; De Pauw, Mervielde, Van Leeuwen, 2009; Measelle, John, Ablow, Cowan, \& Cowan, 2005; Mervielde et al., 1995). In particular, Shiner and DeYoung (2013) have argued that more than five traits are necessary to capture the meaningful variance in child temperament/personality.

In conclusion, extant literature indicates that, while there is support for conceptual overlap between adult personality models and traits and those found in children and adolescents, the extent of this support varies across child age. These inconsistencies in findings may reflect differences in assessing and conceptualizing temperament/personality traits across studies (Caspi \& Shiner, 2006) as well as reliance on small samples and outdated statistical methodologies, suggesting that further research with children is necessary in order to clarify differences in the developmental manifestations of the structure of temperament (Caspi \& Shiner, 2006; De Pauw et al., 2009). Furthermore, investigations of the structure of child temperament traits so far have relied almost exclusively on parent report measures of these constructs. Given the low level of convergence of parent reports of child temperament with other measures such as teacher reports and observational paradigms (e.g., Durbin, Hayden, Klein, \& Olino, 2007; Goldsmith \& Campos, 1990; Rothbart, Derryberry, \& Hershey, 2000), it is important to consider the advantages of parent-report and observational methods in order to understand how these may impact the structures of child temperament they yield.

\section{Methods Used to Assess Child Temperament}

With self-report measures dominating the field of research on adult personality structure, there is little means to address questions regarding structural reliability across methods. In contrast, the question of whether assessment method influences our understanding of child 
temperament structure is open to investigation. While the majority of research on the structure of child temperament has historically relied on parent report methods, observational measures are far more widely used in these studies (Durbin, 2010), meaning that investigators can explore whether structural aspects of child temperament are robust across methods (e.g., Dyson et al., 2012; Kotelnikova, Olino, Mackrell, Jordan, \& Hayden, 2013).

While the relevant literature is small thus far, related work suggests that structural findings on the nature of child temperament are likely to vary as a function of source. More specifically, parent reports typically show low convergent validity with teacher reports and observational and laboratory measures (Durbin et al., 2007). Several authors have shown that parent reports of child behavior are influenced by mood state and availability biases (Durbin et al., 2007; Durbin \& Wilson, 2012; Hayden, Durbin, Klein, \& Olino, 2010; Rothbart \& Bates, 2006), parents'symptoms of psychopathology (e.g., maternal symptoms of anxiety and depression; Durbin \& Wilson, 2012), and personality traits (e.g., lower levels of positive emotionality; Durbin \& Wilson, 2012; Youngstrom, Izard, \& Ackerman, 1999). As a result, it is likely that parent reports of child temperament reflect both objective and subjective information resulting in a structure that is highly influenced by parents' perception of their children.

In light of the concerns surrounding parent reports, a body of research on child temperament assessed using observational methods has emerged. Despite being expensive and time-consuming to administer, laboratory measures of child temperament (e.g., Gagne, Van Hulle, Aksan, Essex, \& Goldsmith, 2011; Goldsmith, Reilly, Lemery, Longley, \& Prescott, 1995) have several advantages, such as reducing rater bias through standardized coding procedures and the use of standardized stimuli. This approach also facilitates observation of child behaviors that may be present at lower frequency rates in naturalistic settings (Durbin et al., 
2007). Therefore, observational measures may confer important advantages over parent reports of child temperament, justifying their cost and time commitment.

Research on the structure of child temperament using observational measures is comprised of two other studies aside from the work presented in the third study of this doctoral thesis. Dyson and colleagues (2012) investigated the structure of observed child temperament in a large sample of preschoolers using a two-step factor analytic approach. The final five-factor model showed only a marginal overlap with the three-factor model of temperament in young children proposed by Rothbart and colleagues (2001), in that three factors of the model derived by Dyson et al. (2012) exhibited some similarity to the Extraversion/Surgency, NA, and EC factors from Rothbart's model. The presence of two additional factors led Dyson et al. (2012) to conclude that some traits such as sociability and positive affect/interest, as well as dysphoria and fear/inhibition, do not coalesce into their respective higher-order factors (Extraversion and Neuroticism) until later in life. Similar findings were obtained by Kotelnikova and colleagues (2013) in a study of observed temperament in middle childhood; here, we recovered a four-factor structure with only a marginal resemblance to the structure proposed by Simonds and Rothbart (2004) for this developmental period. Similar to the study by Dyson et al, 2012, we found that separate factors represented the facets of NA (fear, anger, and sadness). As a result, more work is required to understand the nature of the structure of observed child temperament to help reconcile the differences between the models obtained using caregiver reports and those obtained using observational measures.

\section{Current Studies and Hypotheses}

The overall goal of the three studies that comprise this doctoral thesis is to gain a better understanding of the structure of temperament in early and middle childhood using both parent 
reports and observational measures. The analyses of child temperament structure based on parent report measures are discussed in the first two studies, which examined Rothbart's measures of temperament in early and middle childhood: the CBQ (Rothbart et al., 2001) and the TMCQ (Simonds, 2006; Simonds \& Rothbart, 2004). Both measures are widely used in research on child temperament (e.g., the paper on validation of the CBQ by Rothbart and colleagues published in 2001 has been cited in the extant literature over 900 times). Although some studies have investigated the higher-order structure of the CBQ by performing factor analyses on the lower-order scales derived using a theory-driven, top-down approach (e.g., Rothbart et al., 2001), there is no research addressing the structure of the CBQ at the item level. Similarly, there is very little information on the validation of the TMCQ at either the higher- or lower-order levels. Therefore, the first two studies fill this gap by examining the lower- and higher-order structures of the CBQ and the TMCQ. We start with an exploratory factor analysis of the items in two large samples of preschoolers for the CBQ $\left(N_{3 \text {-year-olds }}=944 ; N_{5 / 6 \text {-year-olds }}=853\right)$ and a large middle childhood sample for the TMCQ $(N$ 9-year-olds $=654)$ in an attempt to recreate the initial scales proposed by Rothbart and colleagues for these two measures (Rothbart et al., 2001; Rothbart \& Simonds, 2004). We then proceed with an exploratory factor analysis of the lower-order factors in order to derive a higher-order structure that can be compared to those initially proposed by Rothbart and colleagues (Rothbart et al., 2001; Rothbart \& Simonds, 2004).

Similarly, we aimed to extend the extremely limited extant literature on the nature and structure of observed child temperament. As noted, other than Dyson et al. (2012) and Kotelnikova et al. (2013), we know of no other published research using standardized observations to characterize the structure of child temperament. Findings from both of these studies suggest both similarities and differences in the structure of temperament in children 
relative to adult personality and relative to the models proposed by Rothbart et al. (Rothbart et al., 2001; Simonds \& Rothbart, 2004). We extended this work by examining observed child temperament in a large sample of preschoolers $(N=409)$, thus providing further clues about possible developmental transitions in the structure of temperament. In addition, since there is no consensus on the nature and number of primary temperament traits in childhood, we wanted to make a contribution to identifying these traits based on a method (i.e., observational measures) that may confer some advantages over informant reports. To attain this goal, we used a combination of exploratory and confirmatory factor analytic techniques by using two independent samples of preschoolers $(N=409$ and $N=559)$. We ran exploratory structural equation modeling (ESEM) analyses in one sample and then attempted to replicate this structure via a confirmatory factor analysis in the second sample. While a clear three-factor structure consisting of Extraversion/Surgency, NA, and EC has been found in several samples of preschoolers based on parent reports (Putnam \& Rothbart, 2006; Rothbart et al., 2001), the paucity of research using observed methods makes formulating a priori hypotheses difficult. Similar to the first two studies, anywhere from three to five factors that bear strong resemblance to existing models of child and/or adult personality and temperament were expected (McCrae \& Costa, 1997; Rothbart et al., 2001; Rothbart \& Simonds, 2004; Tellegen, 1985; Watson \& Clark, 1993). 


\section{References}

Abe, J. A. A. (2005). The predictive validity of the five-factor model of personality with preschool age children: A nine year follow-up study. Journal of Research in Personality, 39, 423-442. doi: 10.1016/j.jrp.2004.05.002

Abe, J. A. A., \& Izard, C. E. (1999). A longitudinal study of emotion expression and personality relations in early development. Journal of Personality and Social Psychology, 77, 566577. doi: $10.1037 / 0022-3514.77 .3 .566$

Ahadi, S. A., Rothbart, M. K., \& Ye, R. (1993). Children's temperament in the US and China: Similarities and differences. European Journal of Personality, 7, 359-377. doi:10.1002/per.2410070506

Allport, G. W. (1937). Personality: A psychological interpretation. New York: Holt, Rinehart, and Winston.

Barbaranelli, C., Caprara, G. V., Rabasca, A., \& Pastorelli, C. (2003). A questionnaire for measuring the big five in late childhood. Personality and Individual Differences, 34, 645664. doi:10.1016/S0191-8869(02)00051-X

Buss, A. H. \& Plomin, R. (1984). Temperament: Early developing personality traits. Hillside, NJ: Erlbaum.

Caspi, A. (2000). The child is the father of the man: Personality continuities from childhood to adulthood. Journal of Personality and Social Psychology, 78, 158-172.

Caspi, A., \& Shiner, R. L. (2006). Personality development. Hoboken, NJ, US: John Wiley \& Sons Inc, Hoboken, NJ.

Clark, L. A. (2005). Temperament as a unifying basis for personality and psychopathology. Journal of Abnormal Psychology, 114, 505-521. doi:10.1037/0021- 
843X.114.4.505

Clark, L. A., \& Watson, D. (1999). In Pervin L. A., John O. P. (Eds.), Temperament: A new paradigm for trait psychology. New York, NY, US: Guilford Press, New York, NY.

Clark, L. A., \& Watson, D. (2008). Temperament: An organizing paradigm for trait psychology Guilford Press, New York, NY.

De Fruyt, F., Mervielde, I., Hoekstra, H. A., \& Rolland, J. (2000). Assessing adolescents' personality with the NEO PI-R. .Assessment, 7, 329-345.

De Pauw, S. S. W., \& Mervielde, I. (2010). Temperament, personality and developmental psychopathology: A review based on the conceptual dimensions underlying childhood traits. Child Psychiatry and Human Development, 41, 313-329. doi:10.1007/s10578-0090171-8

De Pauw, S. S. W., Mervielde, I., \& Van Leeuwen, K. G. (2009). How are traits related to problem behavior in preschoolers? similarities and contrasts between temperament and personality. Journal of Abnormal Child Psychology,37, 309-325. doi: 10.1007/s10802008-9290-0

DeYoung, C. G. (2006). Higher-order factors of the Big Five in a multi-informant sample. Journal of Personality and Social Psychology, 91, 1138-1151.

Digman, J. M. (1997). Higher-order factors of the big five. Journal of Personality and Social Psychology, 73, 1246-1256. doi: 10.1037/0022-3514.73.6.1246

Digman, J. M. (1994). In Costa P. T., Widiger T. A. (Eds.), Historical antecedents of the five-factor model. Washington, DC, US: American Psychological Association, Washington, DC. doi:10.1037/10140-001

Digman, J. M. (1990). Personality structure: Emergence of the five-factor model. Annual Review 
of Psychology, 41, 417-440. doi: 10.1146/annurev.ps.41.020190.002221

Digman, J. M., \& Shmelyov, A. G. (1996). The structure of temperament and personality in Russian children. Journal of Personality and Social Psychology, 71, 341-351. doi:10.1037/0022-3514.71.2.341

Durbin, C. E. (2010). Modeling temperamental risk for depression using developmentally sensitive laboratory paradigms. Child Development Perspectives, 4, 168-173. doi:10.1111/j.1750-8606.2010.00137.x

Durbin, C. E., Hayden, E. P., Klein, D. N., \& Olino, T. M. (2007). Stability of laboratoryassessed temperamental emotionality traits from ages 3 to 7. Emotion, 7, 388-399. doi:10.1037/1528-3542.7.2.388

Durbin, C. E., \& Wilson, S. (2012). Convergent validity of and bias in maternal reports of child emotion. Psychological Assessment, 24, 647-660. doi:10.1037/a0026607

Dyson, M.W., Olino, T.M., Durbin, C.E., Goldsmith, H.H., \& Klein, D. N. (2012). The structure of temperament in preschoolers: A two-stage factor analytic approach. Emotion, 12, 4457.

Eisenberg, N., Guthrie, I. K., Murphy, B. C., Shepard, S. A., Cumberland, A., \& Carlo, G. (1999). Consistency and development of prosocial dispositions: A longitudinal study. Child Development, 70, 1360-1372.

Eisenberg, N., Valiente, C., Spinrad, T. L., Cumberland, A., Liew, J., Reiser, M., . . Losoya, S. H. (2009). Longitudinal relations of children's effortful control, impulsivity, and negative emotionality to their externalizing, internalizing, and co-occurring behavior problems. Developmental Psychology, 45, 988-1008. doi: 10.1037/a0016213

Eysenck, H. J. (1967). The biological basis of personality. Thomas: Springfield, Ill. 
Gagne, J. R., Van Hulle, C. A., Aksan, N., Essex, M. J., \& Goldsmith, H. H. (2011). Deriving childhood temperament measures from emotion-eliciting behavioral episodes: Scale construction and initial validation. Psychological Assessment, 23, 337-353. doi:10.1037/a0021746

Goldberg, L. R. (1993). The structure of phenotypic personality traits. American Psychologists, $48,26-34$.

Goldberg, L. R. (2001). Analyses of Digman's child-personality data: Derivation of big-five factor scores from each of six samples. Journal of Personality, 69, 709-743. doi: $10.1111 / 1467-6494.695161$

Goldsmith, H. H., Buss, A. H., Plomin, R., Rothbart, M. K., Thomas, A., Chess, S., . . McCall, R. B. (1987). What is temperament? Four approaches. Child Development, 58, 505-529.

Goldsmith, H. H., \& Campos, J. J. (1990). The structure of infant temperamental dispositions to experience fear and pleasure: A psychometric perspective. Child Development, 61, 1944-1964.

Goldsmith, H. H., Reilly, J., Lemery, K. S., Longley, S., \& Prescott, A. (1995). Laboratory Temperament Assessment Battery: Preschool version. Unpublished manuscript.

Graziano, W. G., \& Ward, D. (1992). Probing the big five in adolescence: Personality and adjustment during a developmental transition. Journal of Personality, 60, 425-439.

Halverson, C. F., Havill, V. L., Deal, J., Baker, S. R., Victor, J. B., Pavlopoulous, V., .. . Wen, L. (2003). Personality structure as derived from parental ratings of free descriptions of children: The inventory of child individual differences. Journal of Personality, 71, 9951026. doi: $10.1111 / 1467-6494.7106005$ 
Hayden, E. P., Durbin, C. E., Klein, D. N., \& Olino, T. M. (2010). Maternal personality influences the relationship between maternal reports and laboratory measures of child temperament. Journal of Personality Assessment, 92, 586-593. doi:10.1080/ 00223891.2010 .513308

Jang, K. L., McCrae, R. R., Angleitner, A., Riemann, R., \& Livesley, W. J. (1998). Heritability of facet-level traits in a cross-cultural twin sample: Support for a hierarchical model of personality. Journal of Personality and Social Psychology, 74, 1556-1565.

John, O. P., Caspi, A., Robins, R. W., Moffitt, T. E., \& Stouthamer-Loeber, M. (1994). The "little five": Exploring the nomological network of the five-factor model of personality in adolescent boys. Child Development, 65, 160-178.

Klein, D. N., Durbin, C. E., \& Shankman, S. A. (2009). In Gotlib I. H., Hammen C. L. (Eds.), Personality and mood disorders. New York, NY, US: Guilford Press, New York, NY.

Kochanska, G., Murray, K., \& Coy, K. C. (1997). Inhibitory control as a contributor to conscience in childhood: From toddler to early school age. Child Development, 6, 263277.

Kotelnikova, Y., Olino, T. M., Mackrell, S. V. M., Jordan, P. L., \& Hayden, E. P. (2013). Structure of observed temperament in middle childhood. Journal of Research in Personality, 47, 524-532. doi:10.1016/j.jrp.2013.04.013

Laursen, B., Pulkkinen, L., \& Adams, R. (2002). The antecedents and correlates of agreeableness in adulthood. Developmental Psychology, 38, 591-603.

Livesley, W. J., Jang, K., \& Vernon, P. A. (1998). Phenotypic and genetic structure of traits delineating personality disorder. Archives of General Psychiatry, 55, 941-948. 
Markey, P. M., Markey, C. N., Tinsley, B. J., \& Ericksen, A. J. (2002). A preliminary validation of preadolescents' self-reports using the five-factor model of personality. Journal of Research in Personality, 36, 173-181. doi: 10.1006/jrpe.2001.2341

Markon, K. E., Krueger, R. F., \& Watson, D. (2005). Delineating the structure of normal and abnormal personality: An integrative hierarchical approach. Journal of Personality and Social Psychology, 88, 139-157. doi:10.1037/0022-3514.88.1.139

Martin, R. P., Wisenbaker, J., \& Huttunen, M. (1994). Review of factor analytic studies of temperament measure based on the Thomas-Chess structural model: Implications for Big Five. In C. F. Halverson, G. A. Kohnstamm, \& R. P. Martin (Eds.), The developing structure of temperament and personality from infancy adulthood (pp. 157-172). Hillside, NJ: Erlbaum.

Matheny, A. P., Jr. (1989). Children's behavioral inhibition over age and across situations: Genetic similarity for a trait during change. Journal of Personality, 57, 215-235.

McCrae, R. R., \& Costa, P. T. (1997). Personality trait structure as a human universal. American Psychologist, 52, 509-516. doi:10.1037/0003-066X.52.5.509

McCrae, R. R., Costa, P. T., Jr., Del Pilar, G. H., Rolland, J., \& Parker, W. D. (1998). Crosscultural assessment of the five-factor model: The revised NEO personality inventory. Journal of Cross-Cultural Psychology, 29, 171-188.

McCrae, R. R., Yamagata, S., Jang, K. L., Riemann, R., Ando, J., Ono, Y., et al. (2008). Substance and artifact in the higher-order factors of the Big Five. Journal of Personality and Social Psychology, 95, 442-455.

McDevitt, S. C. (1986). Continuity and discontinuity of temperament in infancy and early childhood: A psychometric perspective. In R. Plomin \& J. Dunn (Eds.), The study of 
temperament: Changes, continuities and challenges (pp. 27-38). Hillsdale, NJ: Erlbaum.

Measelle, J. R., John, O. P., Ablow, J. C., Cowan, P. A., \& Cowan, C. P. (2005). Can children provide coherent, stable, and valid self-reports on the big five dimensions? A longitudinal study from ages 5 to 7. Journal of Personality and Social Psychology, 89, 90-106. doi: $10.1037 / 0022-3514.89 .1 .90$

Mervielde, I., Buyst, V., \& De Fruyt, F. (1995). The validity of the big-five as a model for teachers" ratings of individual differences among children aged 4-12 years. Personality and Individual Differences, 18, 525-534.

Nitschke, J. B., Heller, W., Imig, J. C., McDonald, R. P., \& Miller, G. A. (2001). Distinguishing dimensions of anxiety and depression. Cognitive Therapy and Research, 25, 1-22.

Pesonen, A.-K., Raikkonen, K., Keskivaara, P., \& Keltikangas-Jarvinen, L. (2003). Difficult temperament in childhood and adulthood: Continuity from maternal perceptions to selfratings over 17 years. Personality and Individual Differences, 34, 19-31.

Presley, R., \& Martin, R. P. (1994). Toward a structure of preschool temperament: Factor structure of temperament assessment battery for children. Journal of Personality, 62, 415-448.

Putnam, S. P., \& Rothbart, M. K. (2006). Development of short and very short forms of the children's behavior questionnaire. Journal of Personality Assessment, 87, 102-112. doi:10.1207/s15327752jpa8701_09

Roberts, B. W., \& DelVecchio, W. F. (2000). The rank-order consistency of personality traits from childhood to old age: A quantitative review of longitudinal studies. Psychological Bulletin, 126, 3-25. doi:10.1037/0033-2909.126.1.3

Rothbart, M. K. (1981). Measurement of temperament in infancy. Child Development, 52, 
569-578.

Rothbart, M. K., Ahadi, S. A., Hershey, K. L., \& Fisher, P. (2001). Investigations of temperament at three to seven years: The children's behavior questionnaire. Child Development, 72, 1394-1408. doi:10.1111/1467-8624.00355

Rothbart, M.K. \& Bates, J.E. (2006). In Handbook of child psychology: Vol. 3 Social, emotional, and personality development (6th ed.), by Rothbart, Mary K., Bates, John E., 99-166. Hoboken, NJ, US: John Wiley \& Sons Inc.

Rothbart, M. K., Derryberry, D., \& Hershey, K. (2000). Stability of temperament in childhood: Laboratory infant assessment to parent report at seven years. In V. J. Molfese \& D. L. Molfese (Eds.), Temperament and personality development across the life span (pp. 85119). Hillsdale, NJ: Erlbaum.

Saudino, K. J. (2005). Special article: Behavioral genetics and child temperament. Journal of Developmental and Behavioral Pediatrics, 26, 214-223. doi:10.1097/00004703200506000-00010

Shiner R. L. (1998). How shall we speak of children's personality in middle childhood? A preliminary taxonomy. Psychological Bulletin, 124, 308-332.

Shiner, R. L., \& Caspi, A. (2012). Temperament and the development of personality traits, adaptations, and narratives. New York, NY, US: Guilford Press, New York, NY.

Shiner, R. L., \& DeYoung, C. G. (2013). The structure of temperament and personality traits: A developmental perspective. The oxford handbook of developmental psychology, vol. 2: Self and other. (pp. 113-141) Oxford University Press, New York, NY.

Shiner, R. L., Masten, A. S., \& Tellegen, A. (2002). A developmental perspective on personality in emerging adulthood: Childhood antecedents and concurrent adaptation. Journal of 
Personality and Social Psychology, 83, 1165-1177.

Simonds, J. (2006). The role of reward sensitivity and response: Execution in childhood extraversion. Unpublished doctoral dissertation, University of Oregon.

Simonds, J. \& Rothbart, M. K. (2004). The Temperament in Middle Childhood Questionnaire (TMCQ): A computerized self-report measure of temperament for ages 710. Poster session presented at the Occasional Temperament Conference, Athens, GA.

Tackett, J. L., Slobodskaya, H. R., Mar, R. A., Deal, J., Halverson, C. F., Baker, S. R., . . . Besevegis, E. (2012). The hierarchical structure of childhood personality in five countries: Continuity from early childhood to early adolescence. Journal of Personality, 80, 847-879. doi:10.1111/j.1467-6494.2011.00748.x

Tellegen, A. (1985). Structure of mood and personality and their relevance to assessing anxiety, with an emphasis on self-report. In A. H. Tuma \& J. D. Maser (Eds.), Anxiety and the anxiety disorders (pp. 681-706). Hillsdale, NJ: Erlbaum.

Thomas, A., \& Chess, S. (1977). Temperament and development. Brunner/Mazel, Oxford.

Watson, D., \& Clark, L. A. (1993). In Wegner D. M., Pennebaker J. W. (Eds.), Behavioral disinhibition versus constraint: A dispositional perspective. Englewood Cliffs, NJ, US: Prentice-Hall, Inc, Englewood Cliffs, NJ.

Watson, D., Gamez, W., \& Simms, L. J. (2005). Basic dimensions of temperament and their relation to anxiety and depression: A symptom-based perspective. Journal of Research in Personality, 39, 46-66. doi:10.1016/j.jrp.2004.09.006

Watson, D., Kotov, R., \& Gamez, W. (2006). In Krueger R. F., Tackett J. L. (Eds.), Basic dimensions of temperament in relation to personality and psychopathology. New York, NY, US: Guilford Press, New York, NY. 
Watson, D., Wu, K. D., \& Cutshall, C. (2004). Symptom subtypes of obsessive- compulsive Disorder and their relation to dissociation. Journal of Anxiety Disorders, 18, 435-458.

Youngstrom, E., Izard, C., \& Ackerman, B. (1999). Dysphoria-related bias in maternal ratings of children. Journal of Consulting and Clinical Psychology, 67, 905-916.

Zuckerman, M., Kuhlman, D. M., \& Camac, C. (1988). What lies beyond E and N? Factor analyses of scales believed to measure basic dimensions of personality. Journal of Personality and Social Psychology, 54, 96-107. 


\section{Higher- and Lower-Order Factor Analyses of the Children's Behavior Questionnaire in Early and Middle Childhood}

Despite the longstanding interest in temperament (Hergenhahn \& Henley, 2009) and its associations with important outcomes (Clark \& Watson, 2008; Digman, 1994), fundamental questions regarding its structure, developmental progression, and methods of assessment are still debated (DePauw \& Mervielde, 2010; Durbin \& Wilson, 2012; Dyson, Olino, Durbin, Goldsmith, \& Klein, 2012). Much contemporary research has been informed by a model developed by Rothbart (2007) that conceptualizes child temperament in terms of individual differences in emotional reactivity and self-regulation (i.e., the ability to modulate reactive processes). This model is instantiated in the Children's Behavior Questionnaire (CBQ; Rothbart, Ahadi, Hershey, \& Fisher, 2001), a caregiver-report measure developed for assessing temperament in children 3 to 7 years of age. Over the past decade, the CBQ has become one of the most widely used measures of child temperament in the field; for example, Rothbart and colleagues' paper (2001) describing the CBQ's development and validation has been cited over 900 times.

The CBQ was developed using a rational approach to items and scales (Capaldi \& Rothbart, 1992; Derryberry \& Rothbart, 1988; Rothbart et al., 2001). More specifically, the CBQ items were taken from existing temperament questionnaires covering other developmental stages, including the Infant Behavior Questionnaire (Rothbart, 1981) and the Physiological Reactions Questionnaire (a measure of adult temperament; Derryberry \& Rothbart, 1988). Items from these measures were revised to be developmentally appropriate for preschoolers. Next, parents (12 mothers and 3 fathers) were asked to provide feedback on the items' face validity. The authors used these items to form 15 a priori temperament trait scales based on those used in 
the New York Longitudinal Study (Thomas \& Chess, 1977). The measure was then administered to the parents of 262 3-7-year-old children and reduced to 195 items by eliminating items that did not show item-total correlations of at least .20 with the scale on which they were posited to load (Rothbart, Ahadi, \& Hershey, 1994). The structure of the trait scales was examined via principal axis factor analysis, which indicated that three superordinate factors (Surgency, Negative Affectivity or NA, and Effortful Control or EC) accounted for much of the variance tapped by the CBQ (Rothbart et al., 2001). The higher-order Surgency factor consisted of the following scales: Activity Level, High Intensity Pleasure, Impulsivity, and Shyness (reversed). The NA factor consisted of Anger/Frustration, Discomfort, Fear, Sadness, and Soothability/Falling Reactivity (reversed). The EC factor consisted of Attentional Focusing, Inhibitory Control, Low Intensity Pleasure, and Perceptual Sensitivity. The CBQ also includes scales measuring Approach/Positive Anticipation and Smiling/Laughter; however, Smiling/Laughter showed high loadings on both Surgency and EC and Approach/Positive Anticipation loaded on all three factors (Rothbart et al., 2001). As a result, no primary factors were identified for these two scales. Similarly, the Attentional Shifting scale was not assigned to a specific factor due its inconsistent pattern of loadings (Rothbart et al., 1994; 2001).

The CBQ was subsequently administered in several U.S. samples comprised of 149 3year-olds, 516 4-5-year-olds, and 341 6-7-year-olds, with a similar structure at the higher-order level emerging in each sample (Rothbart et al., 2001). Further, the higher-order three-factor structure reported by Rothbart and colleagues (2001) was evident in other samples (Ahadi, Rothbart, \& Ye, 1993; Kochanska, De Vet, Goldman, Murray, \& Putnam, 1994; Richard, Davis, \& Bums, 2008). However, to our knowledge, no study has examined the structure of the full CBQ at the item level. Thus, whether the CBQ items cluster together to form the 15 lower-order 
scales they are postulated to form is unclear. It is also important to note that the full version of the CBQ (Rothbart et al., 2001) is quite lengthy (195 items). While short versions of the CBQ have been developed, these versions were not created based on item-level factor analyses. For example, Putnam and Rothbart (2006) developed short (94 items; 15 scales) and very short (35 items; three broad factors) versions of the CBQ using item-total correlations and within-scale factor analytic procedures for scale item selection. The very short version contains three scales reflecting Surgency, NA, and EC represented by two or three items for each scale (Putnam \& Rothbart, 2006). However, the authors' use of item-total correlations to derive factors yielded factors that are strongly affected by item properties. As a result, these factor structures may be less accurate and difficult to replicate (Goodwin \& Leech, 2006). Indeed, evidence for the validity of these shorter versions has been mixed; for example, the three-factor item-level structure of the very short form of the CBQ showed only a marginal fit to the data (Putnam \& Rothbart, 2006). An item-level analysis of the full version would be a more stringent and empirical means of shortening the CBQ by identifying items that could be dropped without undue loss of information.

There are other gaps in the literature on the CBQ. In particular, there are very few studies testing whether a similar higher-order structure is found in different ages of children. This is noteworthy given that the CBQ was designed to assess child temperament across a fairly broad window of development during early and middle childhood, a period in which rapid developmental changes are known to occur (Blankson, O'Brien, Leerkes, Marcovitch, Calkins, \& Weaver, 2013; Creel, 2012; Welch-Ross, 1995). At the higher-order level, Rothbart et al. (2001) argued that the CBQ's structure did not change from age 3 to age 7 based on an examination of the similarities of factor patterns in separate samples of younger (3-year-olds) and older (6-7- 
year-olds) children, concluding that the CBQ higher-order structure was comparable across time. However, a more stringent approach would be to use item-level exploratory factor analysis (EFA) followed by a scale-level EFA in samples of children that span the age range covered by the CBQ. While research on the structure of child temperament is generally sparse, especially in comparison to the analogous literature on adult temperament/personality (McCrae \& Costa, 2008; Zuckerman, 2011), extant studies suggest that there may be differences in the number and nature of broad dimensions of temperament across this period of development (Dyson et al., 2012; Kotelnikova, Olino, Mackrell, Jordan, \& Hayden, 2013). Thus, investigating whether the CBQ evinces the same structure in samples of children that vary in age is another important step in research on this instrument.

To summarize, an item-level EFA of the CBQ would provide empirically grounded information on the nature of its lower-order scales, as well as identifying any poorly functioning items, and a developmentally informative design would speak to the nature of the CBQ's structure over time. Furthermore, the EFA approach at the higher-order level replicates methods used by Rothbart et al. (2001). We therefore investigated the higher- and lower-order structure of the CBQ in a large community sample of primary caregivers and their children assessed at age 3 $(N=944)$ and followed up three years later $(N=853)$. Conducting parallel analyses at both waves of data (i.e., child ages 3 and 5/6), we first performed an EFA of the CBQ items at the item level, dropping poorly functioning items (i.e., those with loadings $<.40$ ). We then performed a higherorder EFA on the factors obtained from the item-level analyses to examine the broader temperament structure of the $\mathrm{CBQ}$, and whether it was consistent with the three-factor solution obtained by Rothbart et al. (2001). 


\section{Method}

\section{Participants}

Data in this study were collected at two different sites: London, ON, Canada (hereafter referred to as the ON sample) and Long Island, New York, USA (referred to as the NY sample). The data sets described in this project were a part of larger longitudinal studies conducted at each of these sites. At the two waves of data collection, 9443 -year-olds and 853 5/6-year-olds and their mothers participated (see Table 1 for sample descriptive statistics). The two samples were generally similar on participant demographics, suggesting that combining the two datasets for analyses was reasonable; we also compared mean CBQ scale scores for the two samples (see next section). To further verify the appropriateness of combining the two samples, we conducted specific tests of structural invariance, as described later in the paper.

\section{Assessment of Temperament}

Primary caregivers completed the CBQ as a measure of their children's temperament at ages 3 and ages 5/6 at both sites. The standard form of the CBQ consists of 195 items rated on a 7-point Likert scale ranging from 1 (extremely untrue) to 7 (extremely true). Scale means and internal consistency statistics are presented in Table 2, and are comparable to those reported in the extant literature (Carranza, Gonzales-Salinas, \& Ato, 2013; Komsi, Räikkönen, Heinonen, Pesonen, Keskivaara, Järvenpää, et al., 2008; Putnam \& Rothbart, 2006; Rothbart et al., 2001).

\section{Between-sample differences.}

Independent-sample t-tests were conducted to examine mean-level differences in scale scores between the two samples. Ten CBQ scales differed significantly $(p<.05)$ between the samples. The pattern of results showed that primary caregivers in the NY sample tended to rate their children at age 3 as consistently higher on traits than primary caregivers in the ON sample. 
At age 5/6, there were four significant mean differences between the two samples (see Table 2); primary caregivers in the NY sample continued to rate their children as higher on temperament traits than primary caregivers in the ON sample, with the exception of Shyness for which the pattern was reversed. In general, effect sizes for between-sample mean differences on the CBQ scales were quite small except for Fear $(d=.43)$ and Perceptual Sensitivity $(d=.38)$, both at age 3 . Further, mean differences on scale scores do not influence structural analyses (Goodwin \& Leech, 2006). Scale distributions were generally good; the mean skewness value for the ON age 3 sample was -.28 (range -.99-.30) and the mean kurtosis value was .18 (range -.42 -1.95). The mean skewness value for the NY age 3 sample was -.29 (range -.77 - .30) and the mean kurtosis value was .12 (range -.34 - 1.10). The mean skewness value for the ON age 5/6 sample was -.23 (range -.58 - .22) and the mean kurtosis value was .02 (range -.37-.68). The mean skewness for the NY age 5/6 sample was -.20 (range -.59-.35) and the mean kurtosis value was -.02 (range .49-.79).

\section{Statistical Approach}

As a first step, items ${ }^{1}$ were subjected to $\mathrm{EFAs}^{2}$ using Mplus 7 statistical software (Muthen \& Muthen, 1998-2012). For parameter estimation procedures, we used the maximum likelihood robust (MLR) estimator (Muthen \& Muthen, 1998- 2012) and the geomin oblique rotation

\footnotetext{
${ }^{1}$ Item 104 "Tends to say the first thing that comes to mind, without stopping to think about it" was accidently omitted from the questionnaire booklets used during the assessment in NY sample when children were 3 years old.

${ }^{2}$ We also used item-level confirmatory factor analyses (CFAs) in an attempt to validate the original scales created by Rothbart and colleagues (2001). The fit for these lower-order models in both the 3-year-old and 5/6-year-old samples was poor based on the $\mathrm{CFI}\left(\mathrm{CFI} 3\right.$ y.o. sample $=.58$ and $\mathrm{CFI}_{5 / 6}$ y.o. sample $=.31$ ), although RMSEA values were acceptable for both age groups $\left(\right.$ RMSEA $_{3 \text { y.o. sample }}=.04$ and RMSEA 5/6y.o. sample $=.05)$. There were also model estimation issues associated with the latent variable covariance matrix (PSI), which was not positive definite in the 5/6-year-olds. Further details of these analyses are available upon request. These results are presented in the Supplemental tables A and B.
} 
method recommended by Browne (2001). This rotation was used for both higher- and lowerorder factor analyses. The Kaiser-Guttman criterion for factor retention in an EFA indicates that factors with eigenvalues over 1 should be retained. Aside from following this criterion for evaluation of our EFA solutions, we also performed a parallel analysis (O’Connor, 2000) in which we ran a gross simulation with 1000 replications to determine what the eigenvalues would be if there were the same number of cases and variables, but the data were random. If the eigenvalues from our real data were lower than expected due to chance (i.e., those produced from the parallel analysis), then that factor would not be interpreted as capturing any latent traits present in the data.

The obtained lower-order factors were then computed as averages of their corresponding items with loadings of $\geq .40^{3}$. In case of cross-loadings (three at age 3 and four at age 5/6), we assigned items to factors with higher (primary) loadings. Next, to examine the higher-order structure of the CBQ, the obtained lower-order factors were subjected to a series of EFAs extracting three to five factors ${ }^{4}$. The decision to focus on three to five factor models was based on the extant literature on personality and temperament structure (Caspi \& Shiner, 2006; De Pauw \& Mervielde, 2010; McCrae \& Costa, 2008; Rothbart et al., 2001; Simonds \& Rothbart, 2004; Watson \& Clark, 1993), which suggests that most of the variance in both child and adult

\footnotetext{
${ }^{3}$ Although a cut-off of .30 is sometimes used to designate an acceptable loading in EFAs, use of a more stringent cut-off of .40 is also common (Briggs \& MacCallum, 2003; Comrey, 1973; Hogarty, Kromrey, Ferron, \& Hines, 2004). In the current study, the cut-off of .30 produced a greater number of items with high cross-loadings (see Tables 3 and 4), resulting in more poorly differentiated lower-order factors.

${ }^{4} \mathrm{We}$ also used CFAs at the higher-order level in an attempt to validate the original three-factor structure of the original lower-order scales proposed by Rothbart and colleagues (2001). The fit of such models was poor based on all fit statistics in both 3 - and 5/6-year olds $\left(\mathrm{RMSEA}_{3 \text { y.o. sample }}=.14 ; \mathrm{CFI}_{3 \text { y.o. sample }}=\right.$

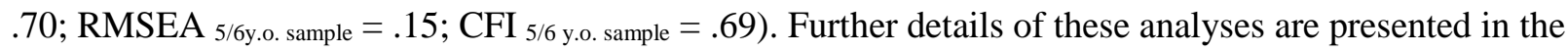
Supplemental Table C.
} 
temperament/personality is accounted for by three to five broad factors (Markon, Kruger, \& Watson, 2005). We used comparative fit index (CFI) values of above .90 and .95 as indices of acceptable and excellent fit (Hu \& Bentler, 1999). Additionally, we treated root-mean-square of approximation (RMSEA) values that were lower than .05 as indicating a close fit, with values up to 0.08 indicating acceptable fit (Marsh, Hau, \& Wen, 2004). Models with varying numbers of factors were compared using the Satorra-Bentler chi-square difference test (Asparouhov \& Muthen, 2009). Due to our large sample size, we could adopt a more stringent test of $p<.01$ for comparisons between models for deciding between different models.

As a final step, we followed a step-wise procedure outlined by Little (2013) to ascertain structural invariance of the higher order solution across the two samples. Thus, models approximating three-, four-, and five-factor solutions obtained at both time points were fitted in a confirmatory factor analysis framework. We subsequently tested for weak, strong, and strict invariance across the two samples (ON and NY). Tests of weak factorial invariance involve setting each corresponding loading in the two samples to be equal; however, variances, intercepts, and residuals are allowed to vary. Testing strong invariance involves imposing equality constraints on each observed intercept across samples, and tests of strict invariance impose equality constraints on residuals across samples (Little, 2013). Higher levels of factorial invariance are acceptable if the change in model fit from a lower level of invariance to a higher level of invariance is negligible, i.e., if the change in RMSEA and CFI does not exceed .015 (Chen, 2007).

\section{Results}

\section{Item-Level Exploratory Factor Analysis at Age 3}

Results of an item-level EFA of the age 3 data in the combined sample are shown in Table 
3. Initially, this analysis identified 54 factors with eigenvalues over 1; however, only 17 factors with larger eigenvalues than the simulated data sets were extracted based on the results of the parallel analysis (O'Connor, 2000). Model fit of the 17-factor EFA solution was deemed good based on the RMSEA (.02); however, the CFI (.85) was weak. Of the 195 items analyzed, 107 items had primary loadings $<.40$, and were excluded from subsequent analyses. Items that were excluded from further analyses largely came from the following original CBQ scales: Low Intensity Pleasure (11 items), Sadness (10 items), Inhibitory Control (10 items), Impulsivity (nine items), Approach/Positive Anticipation (nine items), Discomfort (eight items), Perceptual Sensitivity (eight items), Fear (eight items), and Soothability/Falling Reactivity (five items). Most of the original scales included by Rothbart and colleagues (1994; 2001) consist of 12 to 13 items; thus, it is noteworthy that excluding more than half of the items from these scales suggests that these constructs will not be adequately represented by the resulting measure. Finally, 88 items had primary loadings $\geq .40$. Of the 17 factors extracted, two factors were excluded from further analyses as they consisted of a single item ("Likes sounds of words and nursery rhymes" and "Goes after what he/she wants"). Thus, 15 factors remained for subsequent analyses (Table 3; see also Supplemental Table D for a list of the items excluded from further analyses).

Of these 15 lower-order factors, seven had content that resembled an original CBQ scale 5 (Rothbart et al., 1994; 2001). These included factors containing items measuring (Low) Shyness (16 items; $\alpha=.94$ ), Smiling/Laughter (eight items; $\alpha=.77$ ), (Low) Attentional Focusing (seven items; $\alpha=.75$ ), Soothability/Falling Reactivity (six items; $\alpha=.77$ ), High Intensity Pleasure (five items; $\alpha=.77$ ), (Low) Activity Level (five items; $\alpha=.73$ ), and Approach/Positive Anticipation (four items; $\alpha=.65$ ). Three additional factors contained a mix of items from at least two original

\footnotetext{
${ }^{5}$ We retained the original CBQ scale names for these lower-order factors in order to facilitate comparisons with the scales developed by Rothbart et al. $(1994 ; 2001)$.
} 
CBQ scales; more specifically, our EFA resulted in factors capturing Impulsivity/High Intensity Pleasure (seven items; $\alpha=.76$ ), Anger/Sadness (five items; $\alpha=.76$ ), and Inhibitory Control/Attentional Shifting (five items; $\alpha=.72$ ). Finally, five factors did not tap constructs that mapped clearly onto contemporary developmental theories of child temperament based on the CBQ or other models. One tapped low distress due to physical pain and bruises (four items; $\alpha=.80$ ), anger about going to bed (three items; $\alpha=.75$ ), fear of darkness (two items; $\alpha=.79$ ), fear of loud noises (two items; $\alpha=.20$ ), and noticing changes in clothing and appearances ( 2 items; $\alpha$ $=.83)$.

\section{Item-Level Exploratory Factor Analysis at Age 5/6}

The same data reduction approach was used for the combined sample assessed at age 5/6 (see Table 4). A 17-factor extraction yielded a good fit based on the RMSEA (.03); however, the CFI (.85) was below the minimum recommended value of .90. Of the 195 items analyzed, 108 items had loadings that were $<.40$; a large majority of these items $(82 \%$; $n=88)$ had similarly low loadings in the age 3 EFA. Similar to the results obtained at age 3, many items were excluded from Low Intensity Pleasure (12 items), Sadness (10 items), Inhibitory Control (nine items), Approach/Positive Anticipation (eight items), Perceptual Sensitivity (eight items), Fear (eight items), Soothability/Falling Reactivity (eight items), Discomfort (seven items), and Impulsivity (seven items). 87 items had loadings $\geq .40$; the majority of these $(n=68 ; 78 \%)$ also had loadings $\geq .40$ in the EFA of the age 3 data. Two of the 17 lower-order factors extracted did not have any item loadings $\geq .40$ and were therefore excluded from further analyses (see Supplemental Table E for a list of all items excluded from further analyses).

The 15 remaining factors are presented in Table 4. Of these factors, four resembled original CBQ scales; these included factors with items tapping Smiling/Laughter (eight items; 
$\alpha=.78$ ), Anger/Frustration (six items; $\alpha=.80$ ), Soothability/Falling Reactivity (four items; $\alpha=.72$ ), and Approach/Positive Anticipation (four items; $\alpha=.64$ ). The original High Intensity Pleasure scale split into two factors consisting of items tapping quiet play (four items; $\alpha=.74$ ) and adventurousness (four items; $\alpha=.77$ ). Three factors consisted of a mix of items from several original CBQ scales, including a Sociability factor consisting of Shyness, Impulsivity, Smiling/Laughter, and High Intensity Pleasure items (18 items; $\alpha=.88$ ), a (low) EC factor comprised of Attentional Focusing, Inhibitory Control, Impulsivity, and Activity Level items (16 items; $\alpha=.88$ ), and a (low) Activity Level factor consisting of Activity Level, Attentional Focusing, and Low Intensity Pleasure items (six items; $\alpha=.77$ ). Finally, six factors did not resemble traditional temperament constructs. These included one containing items reflecting being irritated by mistakes (two items; $\alpha=.50$ ), a factor comprised of items tapping fear of darkness (two items; $\alpha=.81$ ), fear of loud noises (two items; $\alpha=.25$ ), low distress by physical pain or bruises (five items; $\alpha=.79$ ), not feeling upset by sad stories (two items; $\alpha=.68$ ), and the tendency to notice changes in clothing and appearances (four items; $\alpha=.82$ ).

Considering the age 3 and age $5 / 6$ results as a whole, many of the items $(n=68,78 \%)$ with acceptable loadings at age 3 also had acceptable loadings at age 5/6. Additionally, the same number of lower-order factors (15) was retained at both ages. Although this number is comparable to the 17 scales proposed by Rothbart et al. (2001), only seven [Smiling/Laughter, (Low) Shyness, (Low) Attentional Focusing, Soothability/Falling Reactivity, High Intensity Pleasure, (Low) Activity Level, and Approach/Positive Anticipation] resembled an original CBQ scale in the age 3 analyses, and only four (Smiling/Laughter, Anger/Frustration, Soothability/Falling Reactivity, and Approach/Positive Anticipation) at age 5/6. Overall, conceptually similar versions of only three original CBQ scales (Smiling/Laughter, 
Soothability/Falling Reactivity, and Approach/Positive Anticipation) were found at both time points in our sample. The remaining lower-order factors extracted at both ages consisted of a mix of items from different original CBQ scales or represented constructs that are not found in contemporary theories of temperament. More specifically, of the lower-order factors consisting of a mix of items from different original scales, only one emerged consistently at both ages. Of the remaining lower-order factors, four tapping constructs not central to temperament (i.e., fear of darkness, fear of loud noises, low distress by physical pain or bruises, and tendency to notice changes in clothing and appearances) were found at both ages.

\section{Higher-Order Exploratory Factor Analysis at Age 3}

The 15 factors identified using the item-level EFA were subjected to a higher-order EFA with a geomin rotation and using MLR estimator. Three to five factors were extracted from the 15 lower-order factors identified at age 3 (Table 3 and Supplemental Table F). A three-factor model yielded a marginally acceptable fit $(\mathrm{RMSEA}=.06 ; \mathrm{CFI}=.91)$. The first factor of this model appeared to tap different facets of EC; the second combined lower-order factors of Anger/Sadness, Soothability/Falling Reactivity, and Inhibitory Control/Attentional Shifting, and the third was consistent with the construct of extraversion/surgency (see Supplemental Table F). Of note, although fit was marginal, this three-factor structure and content resembles the threefactor model original proposed by Rothbart et al. (2001).

A four-factor model (presented in Table 5) yielded a significantly better fit based on the chi-square and CFI difference tests $\left(\Delta \chi^{2}(12)=89.87 ; p<.001 ; \mathrm{RMSEA}=.05 ; \mathrm{CFI}=.95 ; \Delta \mathrm{CFI}=\right.$ .05). The first factor of this model appeared to be consistent with the construct of sensationseeking, as it was comprised of High Intensity Pleasure and Impulsivity. The second factor, comprised largely of Attentional Focusing, Inhibitory Control/Attentional Shifting, Activity 
Level, and Anger/Sadness tapped low EC/disinhibition and some aspects of NA. The third factor primarily reflected low NA, consisting of (Low) Shyness, low Anger/Sadness, and Soothability, while the fourth represented mostly a combination of Smiling/Laughter and Approach/Positive Anticipation (Table 5).

A five-factor model presented in Table 5 yielded a significantly better fit than the fourfactor model based on the chi-square difference and the CFI difference tests $\left(\Delta \chi^{2}(11)=44.19\right.$; $p<.001 ;$ RMSEA $=.05 ; \mathrm{CFI}=.97 ; \Delta \mathrm{CFI}=.02)$. Similar to the four-factor model, the first factor of this model resembled mostly sensation-seeking and the second factor appeared to tap low EC/disinhibition. The third factor appeared to tap NA and aspects of EC, largely consisting of Anger/Sadness and Impulsivity. The fourth factor comprised of soothability and emotion regulation, and the fifth was mostly a combination of Smiling/Laughter and Approach/Positive Anticipation (Table 5).

We also tested for invariance of temperament structures across the two samples (i.e., ON and NY) to determine whether the three-, four-, and five-factor solutions derived in a joint sample are acceptable. Table G of the Supplementary Material outlines the results of structural invariance tests (i.e., weak, strong, and strict) that were applied sequentially to the three-, four-, and five-factor solutions. Results indicated that imposition of weak, strong, and strict invariance of the solutions did not significantly diminish model fit. Thus, the factorial structure of the instrument is equivalent across the two samples.

\section{Higher-Order Exploratory Factor Analysis at Age 5/6}

Three to five factors were extracted from the 15 lower-order factors identified at age 5/6 (Table 4 and Supplemental Table H) in the combined sample. A three-factor model yielded a marginally acceptable fit based on RMSEA of .08, but the CFI of .86 did not reach the cut-off of 
an acceptable fit. This model was similar to the three-factor model obtained at age 3, and thus bore some resemblance to the model of Rothbart and colleagues (2001). Thus, the first factor comprised Anger, (Low) EC, and Soothability/Falling Reactivity, the second appeared to tap lower EC and higher impulsivity, and the third resembled the construct of extraversion/surgency (see Supplemental Table H).

A four-factor model (Table 6) yielded a significantly better fit based on chi-square and CFI difference tests $\left(\Delta \chi^{2}(12)=135.65 ; p<.001 ; \mathrm{RMSEA}=.06 ; \mathrm{CFI}=.94 ; \Delta \mathrm{CFI}=.08\right)$. The first factor of this model could be interpreted as disinhibition/anger, as it consisted of (Low) EC, Anger/Frustration, and Activity Level. The second factor appeared to tap sensation-seeking, as it contained Adventurous and Quiet Play. The third factor was comprised of a combination of Smiling/Laughter and Approach/Positive Anticipation, and the fourth was largely defined by Soothability/Falling Reactivity (see Table 6). A five-factor solution was not admissible due to a negative residual variance for one of the variables and will not be considered further.

Although tests of invariance indicated that strict invariance of the three-factor solution could be assumed across the two samples, the unconstrained model for this solution was only marginally acceptable (Supplemental Table G). As a result, tests of invariance for the three-factor solution should be interpreted with caution. Table I of the Supplementary Materials outlines the differences between the three-factor solutions in the two samples. Tests of invariance also indicated that weak and strong invariance did not reduce the fit of the four-factor solution across the two samples, but strict invariance did reduce model fit (Supplemental Table G). We provide the four-factor solutions for each sample in Supplemental Table J. A five-factor model did not yield an admissible solution in the sample of 5/6-year-olds. 


\section{Discussion}

We used a bottom-up approach to examining the higher- and lower-order structures of a widely used measure of child temperament, the CBQ (Rothbart et al. 1994; 2001). To our knowledge, our item-level analysis of this popular measure is unique in the literature, likely due to the difficulty in acquiring a sufficient sample size for item-level analyses of a measure as lengthy as the CBQ. Given that we had two waves of data on children ages 3 and 5/6, we conducted item-level EFAs at two time points that roughly capture the beginning and end of the developmental time frame covered by the CBQ. Findings indicated that a large number of CBQ items $(55 \%)$ did not clearly differentiate between lower-order factors. Several lower- and higherorder temperament dimensions (e.g., fear and sadness) thought to be important components of temperament in most major models (Caspi \& Shiner, 2006; De Pauw, Mervielde, \& Van Leeuwen, 2009; Rothbart et al., 2001) were poorly represented in the structures derived in our sample, due to purportedly relevant items failing to load onto scales. Finally, the larger structure of child temperament was not well represented by a three-factor solution in our sample, in contrast to the overarching three-factor structure posited by Rothbart and colleagues (2001). We found that a four-factor higher-order structure showed a very good fit and structural invariance across samples at ages 3 and 5/6 in our sample; further, the empirically derived four-factor structures at ages 3 and 5/6 were quite similar; thus, we focus on this model throughout this discussion.

While the CBQ is an especially lengthy measure, our findings indicate that a large number of items do not contribute to lower-order scales. More specifically, EFAs conducted at the item-level indicated that less than half of the original 195 items loaded onto lower-order factors; notably, many of the items that did not load onto a lower-order scale at age $3(82 \%)$ also 
did not load onto a lower-order scale at age 5/6. Item-level analyses yielded only a handful of factors that resembled the original CBQ scales created by Rothbart and colleagues (1994; 2001). At age 3, these included High Intensity Pleasure, (Low) Shyness, (Low) Attentional Focusing, Soothability/Falling Reactivity, Approach/Positive Anticipation, Smiling/Laughter, and (Low) Activity Level; at age 5/6 only the Anger/Frustration, Smiling/Laughter, Approach/Positive Anticipation, and Soothability/Falling Reactivity scales were similar to Rothbart's. In other words, of the 15 lower-order scales in the original CBQ, only seven approximating these emerged from our item-level analyses at age 3, only four at age 5/6, and only three were consistently found at both ages. The remaining factors were comprised of items from multiple scales or did not represent constructs broad enough to be deemed temperament traits (e.g., one scale reflected fear of darkness). In other words, fewer than half of the scales developed by Rothbart and colleagues using a rational approach $(1994 ; 2001)$ were found using a more empirical approach. These findings suggest that the CBQ is longer than necessary and that many of its items are not effective indicators of the constructs they purport to tap.

Relatedly, Putnam and Rothbart (2006) developed two shorter versions of the CBQ by examining the pattern of item-total correlations, scale content, and conducting within-scale factor analyses, rather than through item-level factor analysis (e.g., Volpe, Gadow, Blom-Hoffman, \& Feinberg, 2009) or item-response theory (e.g., Sharp, Steinberg, Temple, \& Newlin, 2014). Several researchers who have examined the short CBQ measures (Allan, Lonigan, \& Wilson, 2013; de la Osa, Granero, Penelo, Domenech, \& Ezpeleta, 2013; Sleddens, Kremers, Candel, De Vries, \& Thijs, 2011) have failed to replicate the higher-order three-factor structure consisting of Surgency, NA, and EC proposed by Rothbart and colleagues (1994; 2001; 2007), raising concerns about the structure of these shortened versions. In particular, Allan et al. (2013) 
concluded that a large number of items of the parent and teacher versions of the very short form of the CBQ did not perform well by showing low convergent and discriminant validity with other widely used measures of child temperament. In the context of the current findings, this may be because the short versions of the CBQ use items that are not good indicators of the posited traits.

As a result of excluding approximately half of the items, several traits held to be important aspects of temperament in children (Caspi \& Shiner, 2006; De Pauw et al., 2009; Rothbart et al., 2001) were not accounted for by the lower-order factors in the structures derived in our sample. At age 3, we found evidence for a (Low) NA factor in the four-factor solution consisting of items tapping sadness and anger, but this factor also included items describing attentional, inhibitory, and perceptual sensitivity aspects of EC. At age 5/6, the NA factor in the four-factor solution similarly consisted of anger and low EC items. Difficulty in deriving clear lower-order fear and sadness factors at ages 3 and 5/6 may be related to the lack of wellfunctioning items that tap these constructs in the current version of the CBQ. At age 3, 10 items $(83 \%)$ from the original Sadness scale and 8 items $(67 \%)$ from the original Fear scale were excluded after the item-level exploratory analyses due to minimal loadings (i.e., <.40) on all lower-order factors. At age 5/6, many of the same fear and sadness items that were dropped from the age 3 analyses were dropped yet again due to low loadings (i.e., 100\% of the fear items and $80 \%$ of the sadness items dropped at age 3 were also dropped at age 5/6). This pattern indicates that the CBQ may benefit from additional work developing sadness and fear items.

Similarly, analyses of lower-order factors showed that current CBQ items also failed to consistently differentiate between the various EC facets identified by Rothbart and colleagues (1994; 2001), which include Attentional Focusing, Attentional Shifting, Inhibitory Control, Low 
Intensity Pleasure, and Perceptual Sensitivity. Although Perceptual Sensitivity was a clearly defined lower-order factor at both ages 3 and 5/6, Low Intensity Pleasure did not replicate as a lower-order factor at either of these ages. Attentional Focusing emerged as a separate lower-order factor and Inhibitory Control items coalesced with Attentional Shifting items in the item-level EFA in 3-year-olds. However, in 5/6-year-olds, items from these three scales coalesced into a single lower-order scale (Table 3 and 4). While literature supports the general notion that EC is a multifaceted construct (Murray \& Kochanska, 2002; Rothbart, Ellis, Rueda, \& Posner, 2003), the CBQ does not appear to consistently differentiate between these facets, suggesting that either EC does not parse into the components the CBQ proposes exist or that revision of the CBQ EC items is needed.

While the four-factor structure found at ages 3 and 5/6 showed some conceptual similarity to that reported by Rothbart and colleagues (2001) (e.g., both contained two affective higher-order factors - an NA-like factor and Smiling/Approach), there were significant differences as well. The structure derived by Rothbart et al (2001) in multiple samples of 3-and 6-year-olds showed a single Surgency factor. Such a factor did not emerge in our analyses, although two dimensions tapping related behaviors did; one of these was characterized by Smiling/Laughter and Approach/Positive Anticipation items, and the other captured behaviors related to sensation-seeking. These findings parallel the development of Big 3 models of personality (Eysenck \& Eysenck, 1985; Tellgen, 1993; Tellegen \& Waller, 2008). Originally, Eysenck's model proposed the existence of two factors, Extraversion and Neuroticism, with Extraversion including a large impulsivity component. He subsequently split the impulsivity/sensation-seeking items off to form a third factor labelled Psychoticism (Eysenck \& Eysenck, 1985). Our findings also parallel the distinction between Positive Emotionality and 
Constraint in the three-factor model and its corresponding measure (Multidimensional Personality Questionnaire) developed by Tellegen and colleagues (Tellegen, 1993; Tellegen \& Waller, 2008).

Similarly, in contrast to Rothbart's three-factor model, we did not recover clearly distinct NA and EC factors at either age 3 or 5/6; instead, we found that scales tapping aspects of EC and NA, particularly anger, clustered together to form higher-order factors (see Table 5, factor 2 for the age 3 solution, Table 6 , factor 1 for the age 5/6 solution. It is possible that differentiation of these factors could be related to developmental changes in the structure of temperament that occur between infancy and early childhood. In a large study of predictors of emerging EC, Gartstein, Slobodskaya, Putnam, and Kinsht (2009) found that infants' NA was a significant predictor of their EC in toddlerhood, suggesting that NA may influence the development of EC, and the possibility that common factors play an etiological role in both constructs. Such etiological overlap may result in less clearly differentiated NA and EC factors in 3-6 year olds. It is also possible that clear NA and EC factors did not emerge in our study due to a significant reduction in the number of items tapping NA and EC scales after our initial item-level EFAs. Given the level of interest on the part of developmental psychologists in these constructs, future work on the CBQ may need to focus on the development of items that successfully tap these constructs.

Also in higher-order EFAs, we recovered factors that resembled Soothability (see Table 5, Factor 3 for the relevant age 3 factor and Table 6 , Factor 4 , for the age 6 factor), which tends to be subsumed under the higher-order NA or Neuroticism factor in extant models of child temperament and personality (Caspi \& Shiner, 2006; De Pauw et al., 2009; Rothbart et al., 2001). In our study, this factor was characterized by items tapping children's ability to recover from 
negative emotions, and may be tapping parent perceptions of children's emotion regulation skills. It is possible that the ease with which a child can be soothed is very salient to parents, which may account for its heightened distinction in our models relative to theoretical accounts of this construct.

One question concerns how well the structural results we obtained relate to findings derived from other approaches to the measurement of child temperament, including observational measures. Two studies (Dyson et al., 2012; Kotelnikova et al., 2013) have addressed the structure of observed temperament in early- and middle-childhood, both of which indicated that more than three factors were needed to adequately capture variance in child temperament. More specifically, Dyson and colleagues (2012) found that a five-factor model consisting of Sociability, Dysphoria (anger, hostility, and sadness), Positive Affect/Interest, Fear/Inhibition, and Impulsivity was the best fit to preschoolers' temperament. The Dysphoria factor derived by Dyson et al. (2012) resembles the lower-order (low) NA factor derived at age 3 in the current study. Further, factors tapping sensation-seeking and positive affect that emerged at both ages 3 and 5/6 in the current study resembled the Impulsivity and Positive Affect/Interest factors, respectively, in Dyson et al.’s (2012) model. Kotelnikova and colleagues (2013) analyzed observed temperament in 7-year-olds, finding a four-factor structure consisting of Positive Emotionality, Disinhibition/Anger, Sadness, and Fear/Behavioral Inhibition factors. In this study, Positive Emotionality and Disinhibition emerged as two separate factors, similar to the current study, in which two dimensions tapping positive affect and sensation-seeking were found. Overall, the structures of temperament derived in our study via a purely empirical approach are somewhat more consistent with models of observed temperament (Dyson et al., 2012; Kotelnikova et al., 2013) than Rothbart and colleagues' (2001) original model. 
Our study is the first item-level analysis of a widely used parent-report measure of temperament in young children. Compared to the analytic methods used in the original scale development (Rothbart et al., 2001), the approach we used is less subject to influence by item properties (Goodwin \& Leech, 2006). The large sample size and multiple waves of CBQ data were significant strengths. In particular, comparing the structure of the CBQ in the same children over time eliminates the confound of age and sample found in cross-sectional studies of different participants who vary in age. However, our study had several limitations. First, the CFI values in our item-level EFA analyses at ages 3 and 5/6 did not reach the recommended value of .90 (Bentler, 1990). However, other fit statistics (i.e., RMSEA) indicated good model fit. Second, despite the acceptable fit coefficients of the higher-order models presented in Tables 5 and 6 , there were relatively few lower-order factors with high loadings. The main implication of the absence of high loading lower-order factors is that the interpretability of the broader factors is somewhat limited; we therefore tried to be agnostic in how we describe these factors throughout the manuscript. Overall, it cannot be said that the higher-order structures capture most of the scales. Although we ascertained structural invariance across the two samples, the four-factor structures derived in the two samples of 5/6-year-olds separately had minor differences (See Supplemental Table J). Also, some of our EFAs included factors with only two items; such factors may not be especially stable or replicate in future analyses. Finally, both samples were racially/ethnically homogenous, which limits the generalizability of our findings to ethnically diverse children.

Our study provides important new information on a widely used measure of child temperament, the CBQ (Rothbart et al., 1994; 2001). The results of our study suggest that revisions of the CBQ are needed, which could include the elimination of poorly functioning 
items, the development of new items to tap important temperamental constructs that may not be currently represented well, and reconsidering the number of factors required to fully represent the domain of temperament in early to middle childhood. Such revisions may greatly benefit researchers in the fields of child development, developmental psychopathology, and temperament assessment. 


\section{References}

Ahadi, S. A., Rothbart, M. K., \& Ye, R. (1993). Children's temperament in the US and China: Similarities and differences. European Journal of Personality, 7, 359-377.

Allan, N. P., Lonigan, C. J., \& Wilson, S. B. (2013). Psychometric evaluation of the Children's behavior questionnaire-very short form in preschool children using parent and teacher report. Early Childhood Research Quarterly, 28, 302-313. doi:10.1016/j.ecresq.2012. 07.009

Asparouhov, T., \& Muthén, B. (2009). Exploratory structural equation modeling. Structural Equation Modeling, 16, 397-438. doi:10.1080/10705510903008204

Bentler, P. M. (1990). Comparative fit indexes in structural models. Psychological Bulletin, 107, 238-246.

Blankson, A. N., O'Brien, M., Leerkes, E. M., Marcovitch, S., Calkins, S. D., \& Weaver, J. M. (2013). Developmental dynamics of emotion and cognition processes in preschoolers. Child Development, 84, 346-360. doi:10.1111/j.1467-8624.2012.01841.x

Briggs, N. E., \& MacCallum, R. C. (2003). Recovery of weak common factors by maximum likelihood and ordinary least squares estimation. Multivariate Behavioral Research, 38, 25-56. doi:10.1207/S15327906MBR3801_2

Browne, M. W. (2001). An overview of analytic rotation in exploratory factor analysis. Multivariate Behavioral Research, 36, 111-150.

Capaldi, D. M., \& Rothbart, M. K. (1992). Development and validation of an early adolescent temperament measure. The Journal of Early Adolescence, 12, 153-173.

Carranza, J. A., González-Salinas, C., \& Ato, E. (2013). A longitudinal study of temperament continuity through IBQ, TBAQ and CBQ. Infant Behavior \& Development, 36, 749-761. 
doi:10.1016/j.infbeh.2013.08.002

Caspi, A., \& Shiner, R. L. (2006). Personality development John Wiley \& Sons Inc, Hoboken, NJ.

Chen, F. F. (2007). Sensitivity of goodness of fit indexes to lack of measurement invariance. Structural Equation Modeling, 14, 464-504.

Clark, L. A., \& Watson, D. (2008). Temperament: An organizing paradigm for trait psychology Guilford Press, New York, NY.

Comrey, A.L. (1973). A first courses in factor analysis. New York: Academic Press.

Creel, S. C. (2012). Preschoolers' use of talker information in on-line comprehension. Child Development, 83, 2042-2056. doi:10.1111/j.1467-8624.2012.01816.x

De la Osa, N., Granero, R., Penelo, E., Domenech, J.M., \& Ezpeleta, L. (2013). The Short and Very Short Forms of the Children's Behavior Questionnaire in a Community Sample of Preschoolers. Assessment, 1, 1-14. doi: 10.1177/1073191113508809

De Pauw, Sarah S. W., \& Mervielde, I. (2010). Temperament, personality and developmental psychopathology: A review based on the conceptual dimensions underlying childhood traits. Child Psychiatry and Human Development, 41, 313-329. doi:10.1007/s10578-009$0171-8$

De Pauw, Sarah S. W., Mervielde, I., \& Van Leeuwen, K. G. (2009). How are traits related to problem behavior in preschoolers? Similarities and contrasts between temperament and personality. Journal of Abnormal Child Psychology,37, 309-325. doi:10.1007/s10802008-9290-0

Derryberry, D., \& Rothbart, M. K. (1988). Arousal, affect, and attention as components of temperament. Journal of Personality and Social Psychology, 55, 958-966. 
doi:10.1037/0022-3514.55.6.958

Digman, J. M. (1994). Historical antecedents of the five-factor model American Psychological Association, Washington, DC. doi:10.1037/10140-001

Durbin, C. E., \& Wilson, S. (2012). Convergent validity of and bias in maternal reports of child emotion. Psychological Assessment, 24, 647-660. doi:10.1037/a0026607

Dyson, M. W., Olino, T. M., Durbin, C. E., Goldsmith, H. H., \& Klein, D. N. (2012). The structure of temperament in preschoolers: A two-stage factor analytic approach. Emotion, 12, 44-57. doi:10.1037/a0025023

Eysenck, H. J., \& Eysenck, S. B. G. (1985). Personality and individual differences. New York: Plenum.

Gartstein, M. A., Slobodskaya, H. R., Putnam, S. P., \& Kinsht, I. A. (2009). A cross-cultural study of infant temperament: Predicting preschool effortful control in the United States of America and Russia. European Journal of Developmental Psychology, 6, 337-364. doi:10.1080/17405620701203846

Goodwin, L. D., \& Leech, N. L. (2006). Understanding correlation: Factors that affect the size of r. Journal of Experimental Education, 74, 251-266. doi:10.3200/JEXE.74.3.249-266

Hergenhahn, B.R. \& Henley, T. (2009). An Introduction to the History of Psychology (6 ${ }^{\text {th }}$ Edition), Cengage Learning.

Hogarty, K. Y., Kromrey, J. D., Ferron, J. M., \& Hines, C. V. (2004). Selection of variables in exploratory factor analysis: An empirical comparison of a stepwise and traditional approach. Psychometrika, 69, 593-611. doi:10.1007/BF02289857

Hu, L., \& Bentler, P. M. (1999). Cut-off criteria for fit indexes in covariance structure analysis: Conventional criteria versus new alternatives. Structural Equation Modeling, 6, 
1-55. doi:10.1080/10705519909540118

Kochanska, G., DeVet, K., Goldman, M., Murray, K., \& Putnam, S. P. (1994). Maternal reports of conscience development and temperament in young children. Child Development, 65, 852-868.

Komsi, N., Räikkönen, K., Heinonen, K., Pesonen, A., Keskivaara, P., Järvenpää, A., \& Strandberg, T. E. (2008). Continuity of father-rated temperament from infancy to middle childhood. Infant Behavior \& Development, 31, 239-254. doi:10.1016/j.infbeh. 2007.10.002

Kotelnikova, Y., Olino, T. M., Mackrell, S. V. M., Jordan, P. L., \& Hayden, E. P. (2013). Structure of observed temperament in middle childhood. Journal of Research in Personality, 47, 524-532. doi:10.1016/j.jrp.2013.04.013

Little, T. D. (2013). Longitudinal structural equation modeling Guilford Press, New York, NY.

Markon, K. E., Krueger, R. F., \& Watson, D. (2005). Delineating the structure of normal and abnormal personality: An integrative hierarchical approach. Journal of Personality and Social Psychology, 88, 139-157. doi:10.1037/0022-3514.88.1.139

Marsh, H. W., Hau, K. T., \& Wen, Z. (2004). In search of golden rules: Comment on hypothesis testing approaches to setting cut-off values for fit indexes and dangers in overgeneralizing Hu \& Bentler's (1999) findings. Structural Equation Modeling, 11, 320341.

McCrae, R. R., \& Costa, P. T., Jr. (2008). Empirical and theoretical status of the five-factor model of personality traits. Sage Publications, Inc, Thousand Oaks, CA.

Murray, K. T., \& Kochanska, G. (2002). Effortful control: Factor structure and relation to externalizing and internalizing behaviors. Journal of Abnormal Child Psychology, 30, 
503-514. doi:10.1023/A:1019821031523

Muthén, L.K. and Muthén, B.O. (1998-2012). Mplus User's Guide. Seventh Edition. Los Angeles, CA: Muthén \& Muthén.

O'Connor, B. P. (2000). SPSS and SAS programs for determining the number of components using parallel analysis and Velicer's MAP test. Behavior Research Methods, Instrumentation, and Computers, 32, 396-402.

Putnam, S. P., \& Rothbart, M. K. (2006). Development of short and very short forms of the children's behavior questionnaire. Journal of Personality Assessment, 87, 102-112. doi:10.1207/s15327752jpa8701_09

Richard, H., Davis, D. W., \& Bums, B. M. (2008). An evaluation of the children's behavior questionnaire for use with children from low-income families. Journal of Early Childhood and Infant Psychology, 4, 111-123.

Rothbart, M. K. (1981). Measurement of temperament in infancy. Child Development, 52, 569-578.

Rothbart, M. K. (2007). Temperament, development, and personality. Current Directions in Psychological Science, 16, 207-212. doi:10.1111/j.1467-8721.2007.00505.x

Rothbart, M. K., Ahadi, S. A., \& Hershey, K. L. (1994). Temperament and social behavior in childhood. Merrill-Palmer Quarterly, 40, 21-39.

Rothbart, M. K., Ahadi, S. A., Hershey, K. L., \& Fisher, P. (2001). Investigations of temperament at three to seven years: The children's behavior questionnaire. Child Development, 72, 1394-1408. doi:10.1111/1467-8624.00355

Rothbart, M. K., Ellis, L. K., Rueda, M. R., \& Posner, M. I. (2003). Developing mechanisms of temperamental effortful control. Journal of Personality, 71, 1113-1143. 
doi:10.1111/1467-6494.7106009

Sharp, C., Steinberg, L., Temple, J., \& Newlin, E. (2014). An 11-item measure to assess borderline traits in adolescents: Refinement of the BPFSC using IRT. Personality Disorders: Theory, Research, and Treatment, 5, 70-78. doi:10.1037/per0000057

Simonds, J. \& Rothbart, M. K. (2004). The Temperament in Middle Childhood Questionnaire (TMCQ): A computerized self-report measure of temperament for ages 710. Poster session presented at the Occasional Temperament Conference, Athens, GA.

Sleddens, E. F. C., Kremers, S. P. J., Candel, M. J. J. M., De Vries, Nanne N. K., \& Thijs, C. (2011). Validating the children's behavior questionnaire in Dutch children: Psychometric properties and a cross-cultural comparison of factor structures. Psychological Assessment, 23, 417-426. doi:10.1037/a0022111

Tellegen, A. (1993). Multidimensional Personality Questionnaire. Minneapolis: University of Minnesota Press.

Tellegen, A., \& Waller, N. G. (2008). Exploring personality through test construction: Development of the multidimensional personality questionnaire Sage Publications, Inc, Thousand Oaks, CA.

Thomas, A., \& Chess, S. (1977). Temperament and development. Brunner/Mazel, Oxford.

Volpe, R. J., Gadow, K. D., Blom-Hoffman, J., \& Feinberg, A. B. (2009). Factor-analytic and individualized approaches to constructing brief measures of ADHD behaviors. Journal of Emotional and Behavioral Disorders, 17, 118-128. doi:10.1177/1063426608323370

Watson, D., \& Clark, L. A. (1993). In Wegner D. M., Pennebaker J. W. (Eds.), Behavioral disinhibition versus constraint: A dispositional perspective. Englewood Cliffs, NJ, US: Prentice-Hall, Inc, Englewood Cliffs, NJ. 
Welch-Ross, M. (1995). Developmental changes in preschoolers' ability to distinguish memories of performed, pretended, and imagined actions. Cognitive Development, 10, 421-441.

Zuckerman, M. (2011). Personality science: Three approaches and their applications to the causes and treatment of depression. American Psychological Association, Washington, DC. doi:10.1037/12309-000 
Table 1.

Sample Descriptive Statistics

\begin{tabular}{|c|c|c|c|c|}
\hline & \multicolumn{2}{|c|}{$\mathrm{ON}$} & \multicolumn{2}{|c|}{ NY } \\
\hline Sample: & Baseline & Follow-up & Baseline & Follow-up \\
\hline$N$ & 406 & 380 & 538 & 473 \\
\hline$M$ child age $(S D)$ & $3.02(.16)$ & $5.44(.50)$ & $3.55(.26)$ & $6.01(1.78)$ \\
\hline$\%$ boys & $49 \%$ & $49 \%$ & $53 \%$ & $54 \%$ \\
\hline$M \operatorname{PPVT}(S D)$ & $112(14)$ & $113(12)$ & $103(14)$ & $108(11)$ \\
\hline $\begin{array}{l}\% \text { of caregivers who } \\
\text { were mothers }\end{array}$ & $94 \%$ & $90 \%$ & $96 \%$ & $91 \%$ \\
\hline$M$ caregiver age $(S D)$ & $33.25(4.62)$ & -- & $35.98(4.35)$ & -- \\
\hline Ethnicity: & & & & \\
\hline Caucasian & $93 \%$ & $93.50 \%$ & $87 \%$ & $79 \%$ \\
\hline African & $.50 \%$ & $.50 \%$ & $1 \%$ & $5 \%$ \\
\hline Asian & $2 \%$ & $2 \%$ & $1 \%$ & $2 \%$ \\
\hline Hispanic/Latino & $2 \%$ & $2 \%$ & $3 \%$ & $5 \%$ \\
\hline Other & $2.5 \%$ & $2 \%$ & $8 \%$ & $9 \%$ \\
\hline Family income: & & & & \\
\hline$<20,000$ & $4 \%$ & $3 \%$ & $1 \%$ & $2 \%$ \\
\hline $20,001-40,000$ & $11 \%$ & $12 \%$ & $4 \%$ & $8 \%$ \\
\hline $40,001-70,000$ & $24 \%$ & $20 \%$ & $21 \%$ & $23 \%$ \\
\hline $70,001-100,000$ & $30 \%$ & $25 \%$ & $35 \%$ & $32 \%$ \\
\hline$>100,000$ & $31 \%$ & $40 \%$ & $39 \%$ & $35 \%$ \\
\hline
\end{tabular}

Note. ON - sample collected in London, ON; NY - sample collected in Long Island, New York, USA. 
Table 2.

Descriptive Statistics for the 15 Original CBQ Scales

\begin{tabular}{|c|c|c|c|c|c|c|c|c|c|c|c|c|c|c|}
\hline \multirow[t]{2}{*}{ Scale } & \multicolumn{2}{|c|}{ ON: age 3} & \multicolumn{4}{|c|}{ NY: age 3} & \multirow[t]{2}{*}{$d$ age 3} & \multicolumn{2}{|c|}{ ON: age $5 / 6$} & \multicolumn{4}{|c|}{ NY: age $5 / 6$} & \multirow[t]{2}{*}{$d$ age $5 / 6$} \\
\hline & $M$ & $S D$ & $\alpha$ & $M$ & $S D$ & $\alpha$ & & $M$ & $S D$ & $\alpha$ & $M$ & $S D$ & $\alpha$ & \\
\hline Activity & 5 & .72 & .77 & 5.02 & .77 & .76 & & 4.74 & .77 & .77 & 4.82 & .73 & .74 & \\
\hline Anger & $4.44 *$ & .77 & .79 & $4.56^{*}$ & .82 & .79 & .15 & 4.25 & .91 & .85 & 4.23 & .88 & .82 & \\
\hline Approach & $5.17 * *$ & .61 & .74 & $5.32 * *$ & .64 & .71 & .24 & 5.20 & .63 & .75 & 5.21 & .63 & .71 & \\
\hline AttnFocus & 4.47 & .79 & .72 & 4.53 & .88 & .72 & & 4.74 & .83 & .76 & 4.73 & .82 & .71 & \\
\hline Discomfort & $4.05^{* * *}$ & .83 & .71 & $4.23^{* *}$ & .84 & .68 & .22 & 4.03 & .88 & .76 & 4.03 & .84 & .70 & \\
\hline Sooth & 5 & .72 & .77 & 5.06 & .75 & .74 & & $5.05 * *$ & .73 & .80 & $5.19 * *$ & .77 & .80 & .19 \\
\hline Fear & $3.62 * *$ & .83 & .71 & $3.98 * *$ & .94 & .74 & .43 & $3.64 * *$ & .93 & .74 & $3.83 * *$ & .95 & .75 & .20 \\
\hline HighPL & $4.95^{* *}$ & .82 & .80 & $5.12 * *$ & .88 & .79 & .20 & 4.93 & .90 & .85 & 4.99 & .81 & .81 & \\
\hline Impulsivity & $4.56^{*}$ & .73 & .79 & $4.67 *$ & .79 & .75 & .14 & 4.41 & .78 & .80 & 4.45 & .74 & .75 & \\
\hline InhibCn & 4.71 & .75 & .79 & 4.61 & .81 & .79 & & 5.10 & .80 & .82 & 5.01 & .84 & .82 & \\
\hline LowPL & $5.65^{*}$ & .56 & .72 & $5.74 *$ & .58 & .69 & .16 & 5.58 & .59 & .73 & 5.53 & .61 & .73 & \\
\hline PerSen & $4.85 * *$ & .75 & .73 & $5.14 * *$ & .77 & .74 & .38 & $4.95^{* *}$ & .75 & .76 & $5.10 * *$ & .71 & .74 & .21 \\
\hline Sadness & $3.79 * *$ & .70 & .68 & $3.95 * *$ & .71 & .63 & .23 & 3.94 & .71 & .66 & 3.86 & .72 & .66 & \\
\hline Shyness & 3.57 & 1.16 & .92 & 3.51 & 1.24 & .92 & & $3.40 *$ & 1.15 & .92 & $3.19 *$ & 1.26 & .93 & .17 \\
\hline Smiling & $5.87 * *$ & .59 & .79 & $6.02 * *$ & .54 & .73 & .27 & 5.90 & .55 & .78 & 5.97 & .54 & .75 & \\
\hline
\end{tabular}

Note. The table depicts between sample comparisons of the CBQ scale means within each age group (3-year-olds and 5/6-year-olds); $* * p<.01 ; * p<.05 ;$ Activity = Activity Level; Anger = Anger/Frustration; Approach = Approach/Positive Anticipation; AttnFocus = Attentional Focusing; Sooth = Soothability/Falling Reactivity; HighPL = High Intensity Pleasure; InhibCn = Inhibitory Control;

LowPL = Low Intensity Pleasure; PerSen = Perceptual Sensitivity; Smiling = Smiling/Laughter 
Table 3.

Exploratory Factor Analysis of the CBQ Items at Age 3

\begin{tabular}{|c|c|c|c|c|c|c|c|c|c|c|c|c|c|c|c|c|c|}
\hline Item\# & Scale & item description & HighPL & LowShy & Imp & NoticeAp & $\begin{array}{l}\text { Low } \\
\text { AttnFocus }\end{array}$ & Ang/Sad & $\begin{array}{c}\text { Ang } \\
\text { AbtBed }\end{array}$ & LowActiv & Smiling & $\begin{array}{l}\text { NotUpset } \\
\text { WPain }\end{array}$ & $\begin{array}{l}\text { InhibCn/ } \\
\text { AttnShift }\end{array}$ & Approach & FearDark & Sooth & $\begin{array}{c}\text { FearOf } \\
\text { LoudNoise }\end{array}$ \\
\hline 60 & HighPL & DoesNotLikeHighSlides & -0.83 & & & NOULCAP & & & & & & & & & & & \\
\hline 8 & HighPL & Adventurous & 0.82 & & & & & & & & & & & & & & \\
\hline 51 & HighPL & DoesNotLikeTakingChances & -0.50 & & & & & & & & & & & & & & \\
\hline 161 & Fear & NotAfraidOfHeights & 0.47 & & & & & & & & & & & & & & \\
\hline 139 & HighPL & LikesToGoHighFastWhenOnSwing & 0.43 & & & & & & & & & & & & & & \\
\hline 129 & Shy & TalksEasilyToNewPeople & & 0.87 & & & & & & & & & & & & & \\
\hline 106 & Shy & ActsShyAroundNewPeople & & -0.82 & & & & & & & & & & & & & \\
\hline 23 & Shy & AtEaseWithAnyone & & 0.79 & & & & & & & & & & & & & \\
\hline 183 & Impulsivity & SlowToWarmToOthers & & -0.77 & & & & & & & & & & & & & \\
\hline 143 & Shy & TurnsAwayShylyFromNewPeople & & -0.75 & & & & & & & & & & & & & \\
\hline 158 & Shy & AtEaseWithAnyGroup & & 0.75 & & & & & & & & & & & & & \\
\hline 57 & Shy & JoinsOthersQuicklyEven|fStranges & & 0.73 & & & & & & & & & & & & & \\
\hline 17 & Shy & ComfortableMeetOthers & & 0.66 & & & & & & & & & & & & & \\
\hline 89 & Shy & NervousWhenTalkingToAdultsJustMet & & -0.61 & & & & & & & & & & & & & \\
\hline 179 & Smiling & SmilesAtFriendlyStrangers & & 0.66 & & & & & & & & & & & & & \\
\hline 71 & Impulsivity & LongTimeInApproachNewSituations & & -0.59 & & & & & & & & & & & & & \\
\hline 45 & Shy & ActsFriendlyWithNewChildren & & 0.58 & & & & & & & & & & & & & \\
\hline 37 & Shy & GetsEmbarrassedWhenStrangersPayAttn & & -0.58 & & & & & & & & & & & & & \\
\hline 119 & Shy & IsComfortableAskingOtherChildrenToPlay & & 0.51 & & & & & & & & & & & & & \\
\hline 74 & Shy & ShyEvenWithPeopleWhoKnowsForLongTime & & -0.50 & & & & & & & & & & & & & \\
\hline 77 & HighPL & EnjoysCrowdsofPeople & & 0.42 & & & & & & & & & & & & & \\
\hline 159 & HighPL & DislikesRowdyGames & -0.32 & & -0.53 & & & & & & & & & & & & \\
\hline 30 & HighPL & DoesNotCareForRoughGames & -0.32 & & -0.52 & & & & & & & & & & & & \\
\hline 25 & Activity & RunsVsWalks & & & 0.52 & & & & & & & & & & & & \\
\hline 22 & HighPL & PlaysWildRecklessly & & & 0.50 & & & & & & & & & & & & \\
\hline 48 & Activity & ClimbsinHouse & & & 0.44 & & & & & & & & & & & & \\
\hline 172 & Activity & FullOfEnergylnTheEvening & & & 0.41 & & & & & & & & & & & & \\
\hline 26 & Impulsivity & InterruptsOthers & & & 0.40 & & & & & & & & & & & & \\
\hline 65 & PerSen & CommentsOnChangesInParentAppearance & & & & 0.85 & & & & & & & & & & & \\
\hline 28 & PerSen & DoesNotCommentOnChangesInAppearance & & & & -0.78 & & & & & & & & & & & \\
\hline 31 & PerSen & NoticesNewClothing & & & & 0.77 & & & & & & & & & & & \\
\hline 38 & AttnFocus & HasAHardTimeKeepingMindOnActiv & & & & & 0.58 & & & & & & & & & & \\
\hline 171 & AttnFocus & EasilyDistractedWhenListeningToStory & & & & & 0.57 & & & & & & & & & & \\
\hline 144 & AttnFocus & BecomesInvolvedWorksForLongPeriods & & & & & -0.56 & & & & & & & & & & \\
\hline 47 & AttnFocus & MovesFromOneTaskToAnotherWithouCompletin & & & & & 0.53 & & & & & & & & & & \\
\hline 126 & Activity & PlaysGamesSlowlyDeliberately & & & & & -0.45 & & & & & & & & & & \\
\hline 195 & AttnFocus & TroublConcentrating WhenDistracting Noises & & & & & 0.44 & & & & & & & & & & \\
\hline 125 & AttnFocus & GoodConcentrationWhenDrawing & & & & & -0.43 & & & & & & & & & & \\
\hline 32 & InhibCn & HardTimeFollowingInstructions & & & & & 0.40 & & & & & & -0.41 & & & & \\
\hline 173 & Anger & EasilylrritatedWhenTroubleWithTask & & & & & & 0.53 & & & & & & & & & \\
\hline 149 & Sadness & RarelyDiscouragedWhenTroubleDoingSmth & & & & & & -0.50 & & & & & & & & & \\
\hline 128 & Anger & EasilyFrustratedWhenTired & & & & & & 0.49 & & & & & & & & & \\
\hline 78 & Anger & AngryWhenCantFindSmthWants ToPlayWith & & & & & & 0.47 & & & & & & & & & \\
\hline 62 & Anger & FustratedWhenPreventedFromDoing Things & & & & & & 0.45 & & & & & & & & & \\
\hline 19 & Anger & RarelylirritatedWhenMakesMistake & & & & & & -0.44 & & & & & & & & & \\
\hline 55 & Sadness & DepressedWhenCannotCompleteTask & & & & & & 0.44 & & & & & & & & & \\
\hline 34 & Anger & HasTemperTantrums & & & & & & 0.42 & & & & & & & & & \\
\hline 193 & Anger & GetsMadWhenProvoked & & & & & & 0.40 & & & & & & & & & \\
\hline
\end{tabular}




$\begin{array}{lll}\text { Item\# } & \text { Scale } & \text { item description } \\ 120 & \text { Anger } & \text { RarelyGetsUpsetWhenToldToGoToBed } \\ 2 & \text { Anger } & \text { AngryWhenAskedtoGotoBed } \\ 103 & \text { Soothability } & \text { FallsAsleepIn10Min } \\ 41 & \text { Activity } & \text { SitsQuietlyOutside } \\ 88 & \text { Activity } & \text { SitsQuitelyForLongPeriods } \\ 36 & \text { LowPL } & \text { EnjoysSittingInSunshine } \\ 192 & \text { Activity } & \text { LikesToSitQuieltyandWatchPpIDoThings } \\ 102 & \text { Activity } & \text { PrefersQuietActivVsGames } \\ 163 & \text { Smiling } & \text { LaughsOutLoudlnPlayWithChildren } \\ 99 & \text { Smiling } & \text { SeldomLaughLoudDuringPlay } \\ 135 & \text { Smiling } & \text { DoesntGiggleOrActSilly } \\ 152 & \text { Smiling } & \text { SmilesAtPeopleWhoLikes } \\ 165 & \text { Smiling } & \text { RarelyLaughsAloudWhenWatchingComedies } \\ 11 & \text { Smiling } & \text { LaughsAtJokes } \\ 153 & \text { Activity } & \text { PlaysActivelyOutdoorsWithChildren } \\ 110 & \text { Smiling } & \text { SmilesGigglesPlayingBySelf } \\ 101 & \text { Discomfort } & \text { NotUpsetByCutsBruises } \\ 61 & \text { Discomfort } & \text { UpsetByALitteCutBruise } \\ 132 & \text { Discomfort } & \text { LikelyToCryWhenABitHurt } \\ 85 & \text { Soothability } & \text { ForgetsBumpsScapesAfterCoupleMin } \\ 168 & \text { InhibCn } & \text { CanEasilyStopWhenToldNo } \\ 29 & \text { AttnShift } & \text { ShiftsEasilyFromOneActivityToAnother } \\ 95 & \text { AttnShift } & \text { TroubleStoppingActivity } \\ 185 & \text { InhibCn } & \text { AbleToResistTemptation } \\ 117 & \text { Approach } & \text { ExcitedBeforeOuting } \\ 96 & \text { Approach } & \text { ExcitedWhenPlanningTrips } \\ 191 & \text { Approach } & \text { LooksFwdFamilyOutingsButNotExcited } \\ 131 & \text { Approach } & \text { CalmBeforeOuting } \\ 46 & \text { Impulsivity } & \text { GoesAfterWhatHe/SheWants } \\ 130 & \text { Fear } & \text { AfraidOfTheDark } \\ 70 & \text { Fear } & \text { NotAfraidOfDark } \\ 134 & \text { Soothability } & \text { EasyToSoothe } \\ 150 & \text { Soothability } & \text { DifficultToSootheWhenUpset } \\ 118 & \text { Soothability } & \text { CheersQuicklylfUpset } \\ 92 & \text { Soothability } & \text { ChangesFeelingsFast } \\ 177 & \text { Soothability } & \text { DoesntCryForMoreThanCoupleMinutes } \\ 68 & \text { Soothability } & \text { WhenAngryUpsetFor10MinOrLonger } \\ 178 & \text { Discomfort } & \text { BotheredByLoudSounds } \\ 50 & \text { Fear } & \text { AfraidOfloudNoises } \\ & & \\ & & \end{array}$

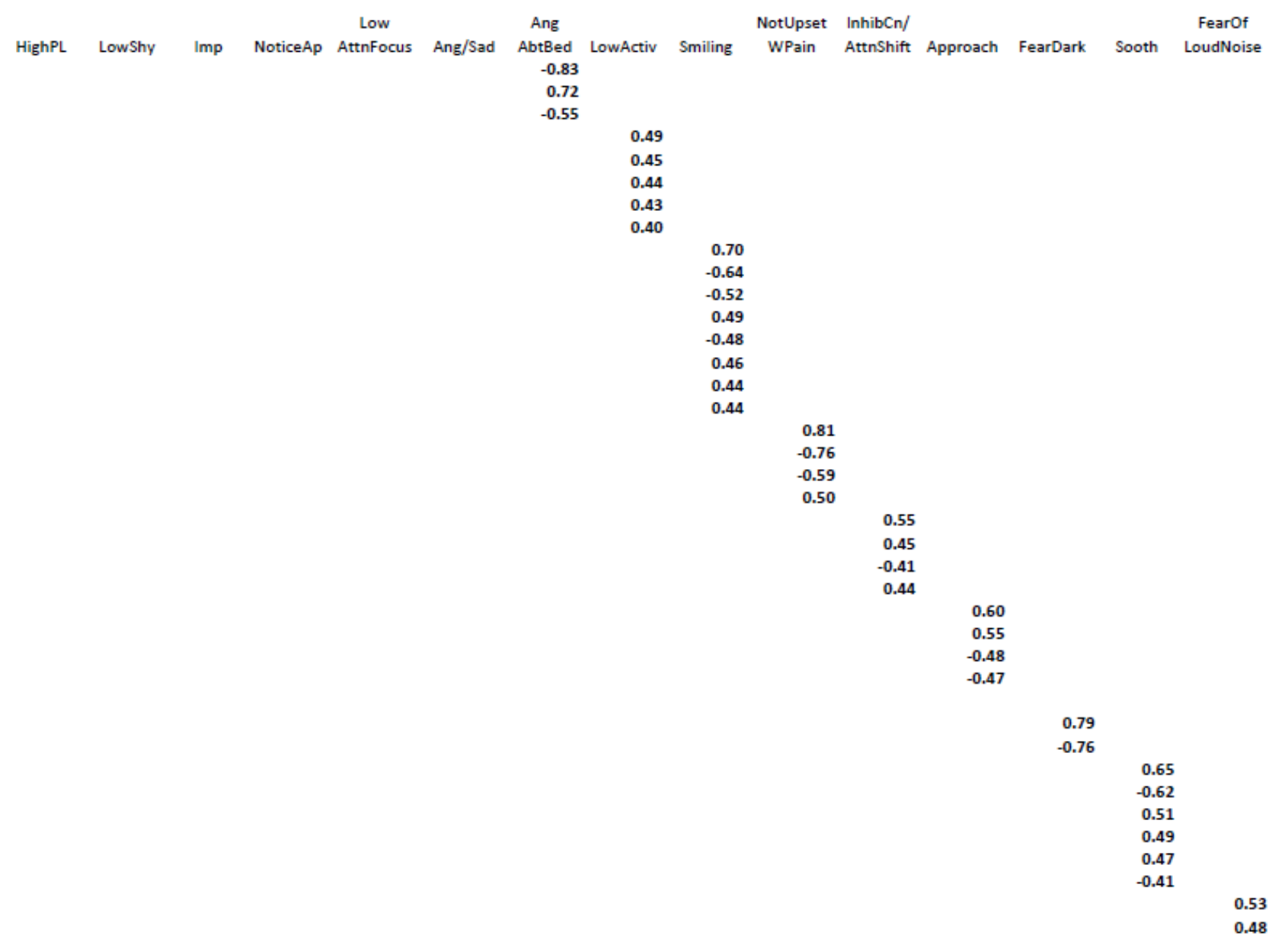

Note. Primary loadings of $\geq .40$ are bolded; secondary loadings of $\geq .30$ were bolded and italicized; two factors had a single item loading on them, i.e., "Like sounds of words and nursery rhymes" and "Goes after what he/she wants", the latter loading was <.40; these two scales were excluded from higher-order exploratory factor analysis; HighPL = High Intensity Pleasure, LowShy = low Shyness, Imp = Impulsivity; NoticeAp = Notices Appearances; LowAttnFoc = low Attentional Focusing; Ang/Sad = Anger and Sadness; AngAbtBed = Anger about going to bed; LowActiv = low Activity Level; Smiling = Smiling/Laughter; NotUpsetWithPain = Not upset by physical pain and bruises; InhibCn/AttnShift = Inhibitory Control/Attentional Shifting; Approach = Approach/Positive Anticipation; FearDark = Fear of darkness; Sooth=Soothability/Falling Reactivity; FearofLoudNoises $=$ Fear of loud noises 
Table 4.

Exploratory Factor Analyses of the CBQ Items at Age 5/6

\begin{tabular}{|c|c|c|c|c|c|c|c|c|c|c|c|c|c|c|c|c|}
\hline Scale & Item description & Anger & Low EC & Sociability & NoticeAp & Advent & QuietP & Smiling & $\begin{array}{l}\text { NotUpset } \\
\text { WPain }\end{array}$ & Approach & $\begin{array}{l}\text { (Low) } \\
\text { Activity }\end{array}$ & $\begin{array}{l}\text { Irritated } \\
\text { Mistakes }\end{array}$ & FearDark & Sooth & $\begin{array}{l}\text { FearOfLoud } \\
\text { Noise }\end{array}$ & $\begin{array}{l}\text { NotUpset } \\
\text { SadStories }\end{array}$ \\
\hline Item Anger & HasTemperTantrums & 0.58 & & & & Ruverio & . & & & approsen & & & Feardarn & Sootn & & \\
\hline 2 Anger & AngryWhenAskedtoGotoBed & 0.57 & & & & & & & & & & & & & & \\
\hline 62 Anger & FustratedWhenPreventedFromDoingThings & 0.53 & & & & & & & & & & & & & & \\
\hline 181 Anger & AngryWhenCalledinFromPlay & 0.45 & & & & & & & & & & & & & & \\
\hline 78 Anger & AngryWhenCantFindSmthWantsToPlayWith & 0.41 & & & & & & & & & & & & & & \\
\hline 95 AttnShift & TroubleStoppingActivity & 0.40 & 0.33 & & & & & & & & & & & & & \\
\hline 38 AttnFocus & HasAHardTimeKeepingMindOnActiv & & 0.75 & & & & & & & & & & & & & \\
\hline 32 InhibCn & HardTimeFollowingInstructions & & 0.75 & & & & & & & & & & & & & \\
\hline 136 Inhibcn & GoodAtFollowinglnstructions & & -0.74 & & & & & & & & & & & & & \\
\hline 47 AttnFocus & MovesFromOneTaskToAnotherWithouCompleting & & 0.74 & & & & & & & & & & & & & \\
\hline 171 AttnFocus & EasilyDistractedWhenListeningToStory & & 0.63 & & & & & & & & & & & & & \\
\hline 195 AttnFocus & TroubiConcentratingWhenDistractingNoises & & 0.54 & & & & & & & & & & & & & \\
\hline 13 Impulsivity & RushesIntoActivWithoutThinking & & 0.54 & & & & & & & & & & & & & \\
\hline 108 InhibCn & TroubleSittingStillWhenTold & & 0.51 & & & & & & & & & & & & & \\
\hline 79 Impulsivity & StopsThinksBeforeDoing & & -0.51 & & & & & & & & & & & & & \\
\hline 125 AttnFocus & GoodConcentration WhenDrawing & & -0.45 & & & & & & & & & & & & & \\
\hline 104 Impulsivity & SaysWhatComestoMind & & 0.45 & & & & & & & & & & & & & \\
\hline 16 AttnFocus & KeepsAtTaskUntilDone & & -0.44 & & & & & & & & & & & & & \\
\hline 184 AttnShift & DoesntSeemToHearWhenTalkedTo & & 0.43 & & & & & & & & & & & & & \\
\hline 144 AttnFocus & BecomesInvolvedWorksForLongPeriods & & -0.42 & & & & & & & & 0.31 & & & & & \\
\hline 126 Activity & PlaysGamesSlowlyDeliberately & & -0.40 & & & & & & & & 0.33 & & & & & \\
\hline 20 InhibCn & GoodAtSimonSays & & -0.40 & & & & & & & & & & & & & \\
\hline 129 Shy & TalksEasilyToNewPeople & & & 0.92 & & & & & & & & & & & & \\
\hline 106 Shy & ActsShyAroundNewPeople & & & -0.85 & & & & & & & & & & & & \\
\hline 183 Impulsivity & SlowToWarmToOthers & & & -0.83 & & & & & & & & & & & & \\
\hline 23 Shy & AtEaseWithAnyone & & & 0.81 & & & & & & & & & & & & \\
\hline 57 Shy & JoinsOthersQuicklyEvenlfStranges & & & 0.77 & & & & & & & & & & & & \\
\hline 158 Shy & AtEaseWithAnyGroup & & & 0.75 & & & & & & & & & & & & \\
\hline 143 Shy & TurnsAwayShylyFrom NewPeople & & & -0.73 & & & & & & & & & & & & \\
\hline 89 Shy & NervousWhenTalkingToAdultsJustMet & & & -0.71 & & & & & & & & & & & & \\
\hline 17 Shy & ComfortableMeetOthers & & & 0.70 & & & & & & & & & & & & \\
\hline 179 Smiling & SmilesAtFriendlyStrangers & & & 0.69 & & & & & & & & & & & & \\
\hline 37 Shy & GetsEmbarrassedWhenStrangersPayAttention & & & -0.62 & & & & & & & & & & & & \\
\hline 45 Shy & ActsFriendlyWithNewChildren & & & 0.62 & & & & & & & & & & & & \\
\hline 71 Impulsivity & LongTimelnApproachNewSituations & & & -0.61 & & & & & & & & & & & & \\
\hline 74 Shy & ShyEvenWithPeopleWhoKnowsForLongTime & & & -0.58 & & & & & & & & & & & & \\
\hline 59 Impulsivity & RushesIntoNewSituations & & & 0.55 & & & & & & & & & & & & \\
\hline 119 Shy & IsComfortableAskingOtherChildrenToPlay & & & 0.53 & & & & & & & & & & & & \\
\hline 77 HighPL & EnjoysCrowdsofPeople & & & 0.44 & & & & & & & & & & & & \\
\hline 7 Shy & PrefersToWatchVsJoinPlaying & & & -0.42 & & & & & & & & & & & & \\
\hline 65 PerSen & CommentsOnChangesInParentAppearance & & & & 0.87 & & & & & & & & & & & \\
\hline 31 PerSen & NoticesNewClothing & & & & 0.82 & & & & & & & & & & & \\
\hline 28 PerSen & DoesNotCommentOnChangesinAppearance & & & & -0.71 & & & & & & & & & & & \\
\hline 98 PerSen & QuicklyAwareOfNewitem InLivingRoom & & & & 0.46 & & & & & & & & & & & \\
\hline 8 HighPL & Adventurous & & & & & 0.70 & & & & & & & & & & \\
\hline 60 HighPL & DoesNotLikeHighSlides & & & & & -0.68 & & & & & & & & & & \\
\hline 139 HighPL & LikesToGoHighFastWhenOnSwing & & & & & 0.50 & & & & & & & & & & \\
\hline 161 Fear & NotAfraidOfHeights & & & & & 0.44 & & & & & & & & & & \\
\hline 51 HighPL & DoesNotLikeTakingChances & & & & & -0.35 & 0.42 & & & & & & & & & \\
\hline
\end{tabular}




\begin{tabular}{|c|c|}
\hline Item\# & Item description \\
\hline 30 HighPL & DoesNotCareForRoughGames \\
\hline 159 HighPL & DislikesRowdyGames \\
\hline 182 HighPL & EnjoysRidingBikeFastRecklessly \\
\hline 163 Smiling & LaughsOutLoudlnPlayWithChildren \\
\hline 99 Smiling & SeldomLaughLoudDuringPlay \\
\hline 152 Smiling & SmilesAtPeopleWholikes \\
\hline 165 Smiling & RarelyLaughsAloudWhenWatchingComedies \\
\hline 135 Smiling & DoesntGiggleOrActSilly \\
\hline 166 Approach & ExcitedWithOpensPresents \\
\hline 153 Activity & PlaysActivelyOutdoorsWithChildren \\
\hline 43 Smiling & EnjoysFunnyStoriesDoesntlaughAtThem \\
\hline 101 Discomfort & NotUpsetByCutsBruises \\
\hline 132 Discomfort & LikelyToCryWhenABitHurt \\
\hline 61 Discomfort & UpsetByALitteCutBruise \\
\hline 85 Soothability & ForgetsBumpsScapesAfterCoupleMin \\
\hline 5 Discomfort & NotVeryBotheredByPain \\
\hline 117 Approach & ExcitedBeforeOuting \\
\hline 96 Approach & ExcitedWhenPlanningTrips \\
\hline 131 Approach & CalmBeforeOuting \\
\hline 191 Approach & LooksFwdFamilyOutingsButNotExcited \\
\hline 192 Activity & LikesToSitQuieltyandWatchPplDoThings \\
\hline 186 AttnFocus & AbsorbedlnPictureBook \\
\hline 36 LowPL & EnjoysSittingInSunshine \\
\hline 88 Activity & SitsQuitelyForLongPeriods \\
\hline 102 Activity & PrefersQuietActivVsGames \\
\hline 41 Activity & SitsQuietlyOutside \\
\hline 19 Anger & RarelylrritatedWhenMakesMistake \\
\hline 173 Anger & EasilyIrritatedWhenTroubleWithTask \\
\hline 130 Fear & AfraidOfTheDark \\
\hline 70 Fear & NotAfraidOfDark \\
\hline 134 Soothability & EasyToSoothe \\
\hline 150 Soothability & DifficultToSootheWhenUpset \\
\hline 118 Soothability & CheersQuicklylfUpset \\
\hline 92 Soothability & ChangesFeelingsFast \\
\hline 178 Discomfort & BotheredByLoudSounds \\
\hline 50 Fear & AfraidOfLoudNoises \\
\hline 112 Sadness & RarelyUpsetWhenWatchingSadTV \\
\hline 109 Sadness & RarelyCriesWhenHearsSadStory \\
\hline
\end{tabular}

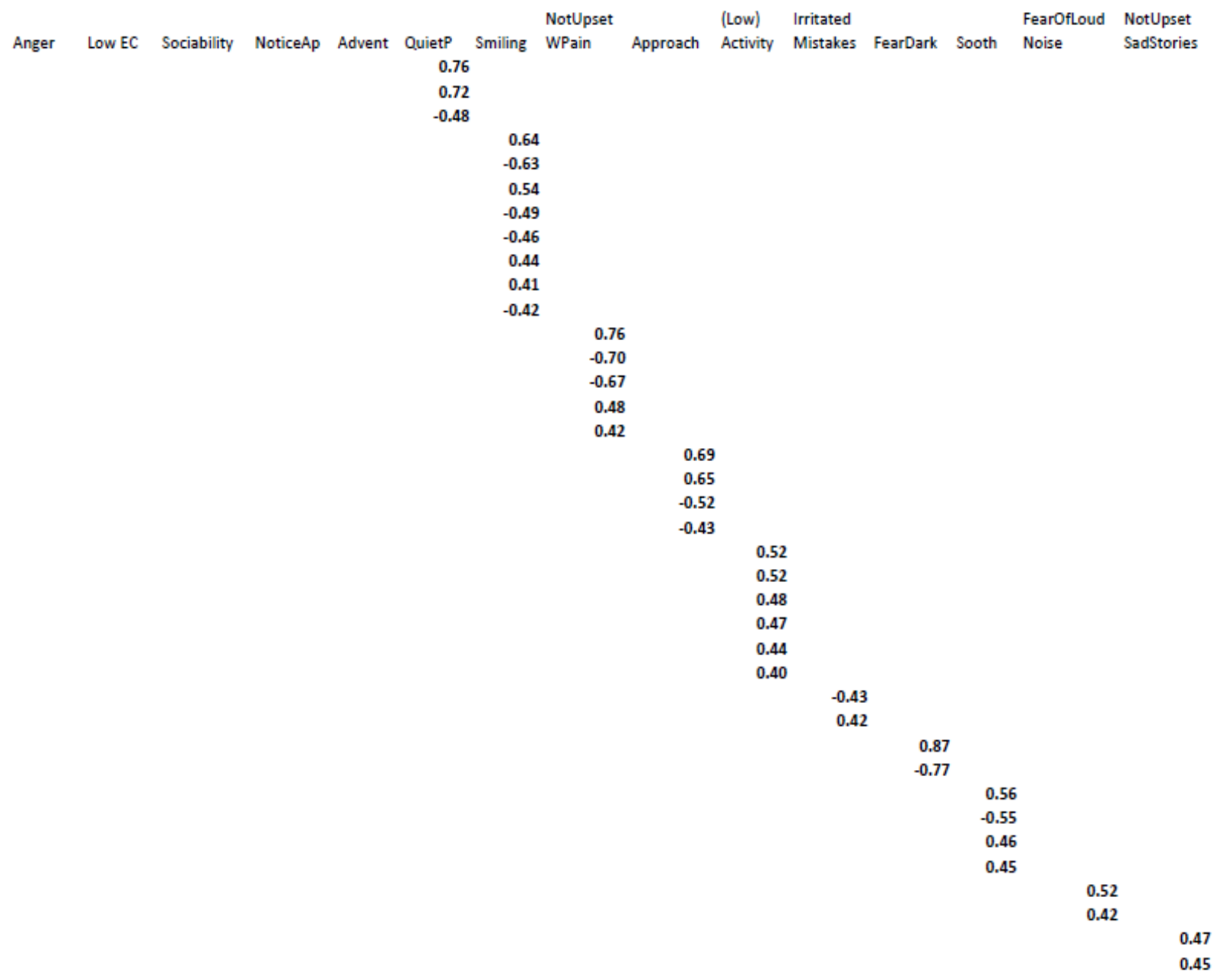

Note. Primary loadings of $\geq .40$ are bolded; secondary loadings of $\geq .30$ are bolded and italicized; two factors had no loadings of $\geq .40$ and were excluded from subsequent analyses; Anger = Anger/Frustration; LowEC = low effortful control; NoticeAp = Notices Appearances; Advent = Adventurous; QuietP $=$ Quiet Play; Smiling= Smiling/Laughter; NotUpsetWithPain = Not upset by physical pain and bruises; Approach = Approach/Positive Anticipation; $($ Low $)$ Activity = low Activity Level; IrritatedMistakes = Irritated by mistakes; FearDark = Fear of darkness; Soothability = Soothability/Falling Reactivity; FearofLoudNoises = Fear of loud noises; NotUpsetSadStories = Not upset by sad stories 
Table 5.

Higher-Order Exploratory Factor Analyses of the CBQ Lower-Order Scales Extracted at Age 3

Four-Factor Solution Five-Factor Solution

\begin{tabular}{|c|c|c|c|c|c|c|c|c|c|c|}
\hline Scales & Factor1 & Factor2 & Factor3 & Factor4 & Scales & Factor1 & Factor2 & Factor3 & Factor4 & Factor5 \\
\hline HighPL & 0.63 & -0.06 & 0.02 & -0.02 & HighPL & 0.58 & -0.09 & 0.00 & 0.03 & 0.01 \\
\hline (Low) Shy & 0.15 & 0.14 & 0.39 & 0.09 & (Low) Shy & 0.16 & 0.17 & -0.04 & 0.34 & 0.14 \\
\hline Impulsivity & 0.61 & 0.41 & -0.03 & 0.05 & Impulsivity & 0.75 & 0.02 & 0.38 & -0.02 & 0.01 \\
\hline NoticeAp & -0.02 & -0.28 & -0.05 & 0.37 & NoticeAp & -0.04 & -0.18 & -0.11 & -0.06 & 0.36 \\
\hline (Low)AttnFocus & -0.04 & 0.66 & 0.03 & -0.12 & (Low) AttnFoc & -0.08 & 0.77 & 0.02 & -0.03 & -0.03 \\
\hline Ang/Sad & -0.01 & 0.37 & -0.37 & 0.23 & Ang/Sad & 0.02 & 0.06 & 0.39 & -0.30 & 0.17 \\
\hline AngAbtBed & -0.06 & 0.38 & -0.17 & 0.05 & AngAbtBed & -0.08 & -0.01 & 0.59 & 0.02 & -0.01 \\
\hline (Low) Activity & -0.22 & -0.46 & -0.19 & -0.01 & (Low) Activity & -0.26 & -0.43 & -0.03 & -0.10 & -0.07 \\
\hline Smiling & 0.18 & -0.02 & 0.30 & 0.47 & Smiling & 0.21 & -0.03 & 0.01 & 0.25 & 0.46 \\
\hline NotUpsetWPain & 0.23 & 0.03 & 0.40 & -0.30 & NotUpsetWPain & 0.26 & 0.05 & -0.08 & 0.36 & -0.26 \\
\hline FearDark & -0.23 & 0.17 & -0.06 & 0.16 & FearDark & -0.23 & -0.01 & 0.30 & 0.04 & 0.11 \\
\hline InhibCn/AttnShift & -0.02 & -0.69 & 0.16 & 0.08 & InhibCn/AttnShift & -0.04 & -0.37 & -0.40 & 0.12 & 0.08 \\
\hline Approach & 0.05 & 0.15 & 0.05 & 0.51 & Approach & 0.04 & 0.18 & 0.00 & -0.01 & 0.57 \\
\hline Soothability & -0.11 & -0.05 & 0.84 & 0.05 & Soothability & -0.06 & 0.01 & -0.05 & 0.81 & 0.06 \\
\hline FearOfLoudNoise & 0.01 & 0.03 & -0.62 & 0.07 & FearOfLoudNoise & -0.02 & 0.09 & -0.09 & -0.68 & 0.08 \\
\hline
\end{tabular}

Note. Primary loadings of $\geq .40$ are bolded; loadings of $\geq .30$ are bolded and italicized; HighPL $=$ High Intensity Pleasure, LowShy $=$ low Shyness, Imp = Impulsivity; NoticeAp = Notices Appearances; LowAttnFoc = low Attentional Focusing; Ang/Sad = Anger and Sadness; AngAbtBed = Anger about going to bed; LowActiv = low Activity Level; Smiling = Smiling/Laughter; NotUpsetWithPain = Not upset by physical pain and bruises; InhibCn/AttnShift = Inhibitory Control/Attentional Shifting; Approach = Approach/Positive Anticipation; Soothability = Soothability/Falling Reactivity; FearDark = Fear of darkness; FearofLoudNoises = Fear of loud noises; in the four-factor solution, correlations between Factor 1 and Factors 2, 3, and 4 were .34, -.24, and .02 respectively, correlations between Factor 2 and Factors 3 and 4 were .05 and - .28 respectively, and the correlation between Factor 3 and 4 was -.26; in the five-factor solution, Factor 1 correlated with Factors 2, 3, 4, and 5 at .32, .02, .38 and .25 respectively; Factor 2 correlated with Factors 3, 4, and 5 at .21, .14, and .29 respectively; Factor 3 correlated with Factors 4 and 5 at -.31 and -.07 respectively, and Factor 4 correlated with Factor 5 at -.01. 
Table 6.

Higher-Order Exploratory Factor Analysis of the CBQ Lower-Order Scales Extracted at Age 5/6

\begin{tabular}{lrrrr}
\hline Scales & Factor1 & \multicolumn{1}{l}{ Factor2 } & Factor3 & Factor4 \\
\hline Anger & $\mathbf{0 . 6 8}$ & -0.04 & -0.01 & -0.17 \\
(Low) EC & $\mathbf{0 . 7 4}$ & 0.05 & $-\mathbf{0 . 3 3}$ & 0.02 \\
Sociability & 0.15 & 0.24 & 0.05 & $\mathbf{0 . 3 5}$ \\
NoticeAp & -0.09 & 0.00 & $\mathbf{0 . 5 6}$ & 0.04 \\
Advent & -0.03 & $\mathbf{0 . 6 7}$ & 0.11 & -0.01 \\
QuietP & -0.23 & $-\mathbf{0 . 6 9}$ & 0.01 & 0.03 \\
Smiling & 0.00 & 0.27 & $\mathbf{0 . 4 6}$ & 0.20 \\
NotUpsetWPain & -0.15 & 0.25 & -0.29 & 0.28 \\
Approach & 0.26 & 0.17 & $\mathbf{0 . 3 9}$ & 0.03 \\
(Low) Activity & $-\mathbf{0 . 3 1}$ & -0.27 & 0.09 & -0.04 \\
IrritatedMistakes & $\mathbf{0 . 4 6}$ & -0.06 & 0.14 & -0.17 \\
FearDark & 0.21 & -0.24 & 0.08 & -0.04 \\
Soothability & 0.02 & -0.22 & 0.05 & $\mathbf{0 . 9 3}$ \\
FearOfLoudNoise & 0.16 & -0.03 & 0.11 & $-\mathbf{0 . 6 4}$ \\
NotUpsetSadStories & 0.11 & 0.01 & $\mathbf{- 0 . 3 6}$ & 0.01 \\
\hline
\end{tabular}

Note. Primary loadings $\geq .40$ are bolded; loadings $\geq .30$ are bolded and italicized; Anger = Anger/Frustration; LowEC $=$ low effortful control; NoticeAp = Notices Appearances; Advent = Adventurous; QuietP = Quiet Play; Smiling= Smiling/Laughter; NotUpsetWithPain = Not upset by physical pain and bruises; Approach = Approach/Positive Anticipation; (Low) Activity = low Activity Level; IrritatedMistakes = Irritated by mistakes; FearDark = Fear of darkness; Soothability = Soothability/Falling Reactivity; FearofLoudNoises = Fear of loud noises;

NotUpsetSadStories = Not upset by sad stories; Factor 1 correlated with Factors 2, 3, and 4 at -.13, .26, and -.09 respectively; Factor 2 correlated with Factors 3 and 4 at .03 and -.22 respectively, and Factor 3 correlated with Factor 4 at .17. 


\section{Supplementary Material}

Table A

Lower-Order CFA Results for Original CBQ Scales at Age 3

\begin{tabular}{|c|c|c|c|}
\hline Scale & Item \# & Abbreviated Item Description & $\begin{array}{l}\text { Standardized } \\
\text { Loadings }\end{array}$ \\
\hline \multicolumn{4}{|l|}{ Activity } \\
\hline & 1 & Seems Always In A Hurry & -0.43 \\
\hline & 25 & Runs Vs Walks & -0.61 \\
\hline & 41 & Sits Quietly Outside & 0.42 \\
\hline & 48 & Climbs In House & -0.53 \\
\hline & 88 & Sits Quietly For Long Periods & 0.34 \\
\hline & 102 & Prefers Quiet Activities Vs Games & 0.65 \\
\hline & 123 & Rarely Runs Moves Quickly In House & 0.40 \\
\hline & 126 & Plays Games Slowly Deliberately & 0.46 \\
\hline & 145 & Sits Quietly In Bath & 0.34 \\
\hline & 153 & Plays Actively Outdoors With Children & -0.32 \\
\hline & 172 & Full Of Energy In The Evening & -0.50 \\
\hline & 187 & Difficulty Sitting Still At Dinner & -0.37 \\
\hline & 192 & Likes To Sit Quietly and Watch People Do Things & 0.57 \\
\hline \multicolumn{4}{|l|}{ Anger } \\
\hline & 2 & Angry When Asked to Go to Bed & 0.54 \\
\hline & 19 & Rarely Irritated When Makes Mistake & -0.29 \\
\hline & 34 & Has Temper Tantrums & 0.64 \\
\hline & 62 & Frustrated When Prevented From Doing Things & 0.64 \\
\hline & 73 & Gets Mad When Mildly Criticized & 0.42 \\
\hline & 78 & Angry When Can't Find Smth. Wants To Play With & 0.52 \\
\hline & 120 & Rarely Gets Upset When Told To Go To Bed & -0.46 \\
\hline & 128 & Easily Frustrated When Tired & 0.49 \\
\hline & 140 & Irritable When Asked To Eat Food Doesn't Like & 0.35 \\
\hline & 156 & Rarely Protests When Another Child Takes Toy & -0.27 \\
\hline & 173 & Easily Irritated When Trouble With Task & 0.54 \\
\hline & 181 & Angry When Called In From Play & 0.51 \\
\hline & 193 & Gets Mad When Provoked & 0.38 \\
\hline \multicolumn{4}{|l|}{ Approach } \\
\hline & 10 & Gets Worked Up \& Has Trouble Sitting Still & 0.41 \\
\hline & 24 & Excited About Getting Toy & 0.49 \\
\hline & 35 & Wants To Do Smth. Talks About Little Else & 0.39 \\
\hline & 69 & Strong Desires For Certain Foods & 0.28 \\
\hline & 82 & Looks Forward To Visits Of Loved Relatives & 0.39 \\
\hline
\end{tabular}




\begin{tabular}{|c|c|c|c|}
\hline & 96 & Excited When Planning Trips & 0.54 \\
\hline & 117 & Excited Before Outing & 0.71 \\
\hline & 131 & Calm Before Outing & -0.48 \\
\hline & 148 & Gets Enthusiastic About Things & 0.50 \\
\hline & 166 & Excited When Opens Presents & 0.46 \\
\hline & 175 & Is Not Excited About TV Programs & -0.30 \\
\hline & 188 & Remains Calm About Desserts & -0.34 \\
\hline & 191 & Looks Forward Family Outings But Not Excited & -0.52 \\
\hline \multicolumn{4}{|l|}{ AttnFocus } \\
\hline & 16 & Keeps At Task Until Done & -0.48 \\
\hline & 38 & Has A Hard Time Keeping Mind On Activity & 0.65 \\
\hline & 47 & Moves From One Task To Another Without Completing & 0.65 \\
\hline & 125 & Good Concentration When Drawing & -0.44 \\
\hline & 144 & When Becomes Involved Works For Long Periods & -0.46 \\
\hline & 160 & Difficulty Leaving Project & -0.14 \\
\hline & 171 & Easily Distracted When Listening To Story & 0.60 \\
\hline & 186 & Absorbed In Picture Book & -0.35 \\
\hline & 195 & Trouble Concentrating When Distracting Noises & 0.50 \\
\hline \multicolumn{4}{|l|}{ AttnShift } \\
\hline & 6 & Hard To Get Attention When Concentrating & 0.49 \\
\hline & 29 & Shifts Easily From One Activity To Another & -0.41 \\
\hline & 95 & Trouble Stopping Activity & 0.70 \\
\hline & 180 & Easy Time Leaving Play To Come To Dinner & -0.40 \\
\hline & 184 & Doesn't Seem To Hear When Talked To & 0.59 \\
\hline \multicolumn{4}{|l|}{ Discomfort } \\
\hline & 5 & Not Very Bothered By Pain & -0.44 \\
\hline & 21 & Uncomfortable Cold Wet & 0.27 \\
\hline & 61 & Upset By A Little Cut Bruise & 0.78 \\
\hline & 87 & Bothered By Light Or Bright Colors & 0.24 \\
\hline & 97 & Finds Rough Materials Uncomfortable & 0.22 \\
\hline & 101 & Not Upset By Cuts Bruises & -0.77 \\
\hline & 115 & Bothered By Bath Too Hot Cold & 0.22 \\
\hline & 132 & Likely To Cry When A Bit Hurt & 0.68 \\
\hline & 141 & Distressed When Hair Combed & 0.27 \\
\hline & 157 & Cries When Given Injection & 0.22 \\
\hline & 178 & Bothered By Loud Sounds & 0.25 \\
\hline & 190 & Seldom Complains When Sick & -0.32 \\
\hline \multicolumn{4}{|l|}{ Soothability } \\
\hline & 14 & Hard Time Settling For A Nap & -0.27 \\
\hline & 27 & Calms Quickly After Exciting Event & 0.44 \\
\hline
\end{tabular}




\begin{tabular}{|c|c|c|c|}
\hline & 42 & Can Be Cheered Up By Talking About Smth. Interesting & 0.25 \\
\hline & 53 & Hard Time Settling Down After Exciting Activity & -0.40 \\
\hline & 68 & When Angry Upset For 10 Min. Or Longer & -0.56 \\
\hline & 85 & Forgets Bumps Scrapes After Couple Min. & 0.31 \\
\hline & 92 & Changes Feelings Fast & 0.45 \\
\hline & 103 & Falls Asleep In 10 Min. & 0.29 \\
\hline & 118 & Cheers Quickly If Upset & 0.58 \\
\hline & 134 & Easy To Soothe & 0.74 \\
\hline & 150 & Difficult To Soothe When Upset & -0.69 \\
\hline & 167 & Hard Time Going Back To Sleep After Waking & -0.33 \\
\hline & 177 & Doesn't Cry For More Than Couple Minutes & 0.55 \\
\hline \multicolumn{4}{|l|}{ Fear } \\
\hline & 15 & Not Afraid Of Large Dogs & -0.14 \\
\hline & 40 & Afraid Of Burglars Boogie Man & 0.43 \\
\hline & 50 & Afraid Of Loud Noises & 0.32 \\
\hline & 58 & Doesn’t Worry About Injections & -0.23 \\
\hline & 70 & Not Afraid Of Dark & -0.71 \\
\hline & 80 & Afraid Of Fire & 0.32 \\
\hline & 91 & Frightened By Nightmares & 0.48 \\
\hline & 130 & Afraid Of The Dark & 0.77 \\
\hline & 138 & Rarely Frightened By Monsters On TV & -0.42 \\
\hline & 161 & Not Afraid Of Heights & -0.25 \\
\hline & 176 & Not Afraid To Sleep Alone & -0.40 \\
\hline & 189 & Nervous About Dentist & 0.23 \\
\hline \multicolumn{4}{|l|}{ HighPL } \\
\hline & 8 & Adventurous & 0.72 \\
\hline & 22 & Plays Wild Recklessly & 0.51 \\
\hline & 30 & Does Not Care For Rough Games & -0.59 \\
\hline & 51 & Does Not Like Taking Chances & -0.65 \\
\hline & 60 & Does Not Like High Slides & -0.69 \\
\hline & 67 & Enjoys Being Chased Spun & 0.41 \\
\hline & 77 & Enjoys Crowds of People & 0.34 \\
\hline & 100 & Enjoys Exciting Suspenseful TV & 0.32 \\
\hline & 107 & Enjoys Santa Other Strangers In Costumes & 0.18 \\
\hline & 124 & Enjoys Exploring New Places & 0.45 \\
\hline & 139 & Likes To Go High Fast When On Swing & 0.43 \\
\hline & 159 & Dislikes Rowdy Games & -0.62 \\
\hline & 182 & Enjoys Riding Bike Fast Recklessly & 0.46 \\
\hline \multicolumn{4}{|l|}{ Impulsivity } \\
\hline & 13 & Rushes Into Activity Without Thinking & 0.43 \\
\hline
\end{tabular}




\begin{tabular}{|c|c|c|c|}
\hline & 26 & Interrupts Others & 0.28 \\
\hline & 46 & Goes After What He/She Wants & 0.43 \\
\hline & 59 & Rushes Into New Situations & 0.69 \\
\hline & 71 & Long Time In Approaching New Situations & -0.65 \\
\hline & 79 & Stops Thinks Before Doing & -0.33 \\
\hline & 90 & Slow At Deciding What To Do & -0.32 \\
\hline & 104 & Says What Comes to Mind & 0.34 \\
\hline & 114 & When Eager To Go Outside/Rushes & 0.26 \\
\hline & 137 & Approaches Slowly Places Where Might Get Hurt & -0.43 \\
\hline & 155 & Eager To Have A Toy Game Right Then & 0.23 \\
\hline & 169 & Does Not Try New Activity & -0.63 \\
\hline & 183 & Slow To Warm To Others & -0.67 \\
\hline \multicolumn{4}{|l|}{ InhibCn } \\
\hline & 4 & Can Lower Voice When Asked & 0.33 \\
\hline & 20 & Good At Simon Says & 0.38 \\
\hline & 32 & Hard Time Following Instructions & -0.68 \\
\hline & 63 & Plans For Trips Outings & 0.28 \\
\hline & 75 & Can Wait When Asked To & 0.35 \\
\hline & 93 & Difficulty Waiting In Line & -0.60 \\
\hline & 108 & Trouble Sitting Still When Told & -0.57 \\
\hline & 116 & Able To Resist Inappropriate Laughing & 0.27 \\
\hline & 136 & Good At Following Instructions & 0.69 \\
\hline & 147 & Cautiously Approaches Dangerous Places & 0.40 \\
\hline & 162 & Not Careful In Crossing Streets & -0.35 \\
\hline & 168 & Can Easily Stop When Told "No" & 0.64 \\
\hline & 185 & Able To Resist Temptation & 0.57 \\
\hline \multicolumn{4}{|l|}{ LowPL } \\
\hline & 12 & Does Not Enjoy Being Talked To & -0.35 \\
\hline & 36 & Enjoys Sitting In Sunshine & 0.39 \\
\hline & 54 & Enjoys Baths & 0.20 \\
\hline & 66 & Doesn't Like Being Read To & -0.44 \\
\hline & 76 & Enjoys Snuggle To Parent Or Babysitter & 0.48 \\
\hline & 86 & Doesn't Like Quiet Games & -0.44 \\
\hline & 111 & Is Not Interested In Quiet TV & -0.30 \\
\hline & 113 & Enjoys Being Talked To & 0.52 \\
\hline & 133 & Enjoys Picture Books & 0.53 \\
\hline & 146 & Likes Being Sung To & 0.48 \\
\hline & 151 & Likes Sounds Of Words Nursery Rhymes & 0.59 \\
\hline & 164 & Enjoys Rhythmic Activities As Rocking & 0.34 \\
\hline & 174 & Enjoys Sitting On Parents Lap & 0.50 \\
\hline
\end{tabular}




\begin{tabular}{|c|c|c|c|}
\hline \multicolumn{4}{|l|}{ PerSen } \\
\hline & 9 & Notices Smoothness Roughness & 0.26 \\
\hline & 28 & Does Not Comment On Changes In Appearance & -0.73 \\
\hline & 31 & Notices New Clothing & 0.76 \\
\hline & 52 & Listens To Quiet Sounds & 0.32 \\
\hline & 65 & Comments On Changes In Parent Appearance & 0.84 \\
\hline & 84 & Doesn't Comment On Facial Features & -0.25 \\
\hline & 98 & Quickly Aware Of New Item In Living Room & 0.43 \\
\hline & 105 & Comments On Unusual Voice & 0.32 \\
\hline & 122 & Does Not Notice Parents' Facial Expressions & -0.38 \\
\hline & 142 & Doesn't React To Food Textures & -0.10 \\
\hline & 154 & Notices Specks of Dirt & 0.33 \\
\hline & 170 & Doesn't Notice Odors Perfume Smoke Cooking & -0.36 \\
\hline \multicolumn{4}{|l|}{ Sadness } \\
\hline & 18 & Cries Sadly When Toy Broken & 0.37 \\
\hline & 39 & Feels Down At The End Of An Exciting Day & 0.35 \\
\hline & 44 & Becomes Sad When Plans Don't Work Out & 0.50 \\
\hline & 55 & Depressed When Cannot Complete Task & 0.51 \\
\hline & 64 & Gets Upset When Relatives Leave & 0.36 \\
\hline & 72 & Doesn't Become Tearful When Tired & -0.33 \\
\hline & 81 & Feelings Easily Hurt By What Parents Say & 0.37 \\
\hline & 94 & Tearful When Told To Do Smth. Does Not Want To Do & 0.47 \\
\hline & 109 & Rarely Cries When Hears Sad Story & -0.20 \\
\hline & 112 & Rarely Upset When Watching Sad TV & -0.13 \\
\hline & 127 & Appears Downcast For No Reason & 0.36 \\
\hline & 149 & Rarely Discouraged When Trouble Doing Smth. & -0.34 \\
\hline \multicolumn{4}{|l|}{ Shyness } \\
\hline & 7 & Prefers To Watch Vs. Join Playing & -0.55 \\
\hline & 17 & Comfortable Meet Others & 0.68 \\
\hline & 23 & At Ease With Anyone & 0.77 \\
\hline & 37 & Gets Embarrassed When Strangers Pay Attention & -0.55 \\
\hline & 45 & Acts Friendly With New Children & 0.72 \\
\hline & 57 & Joins Others Quickly Even If Strangers & 0.79 \\
\hline & 74 & Shy Even With People Who Knows For Long Time & -0.52 \\
\hline & 89 & Nervous When Talking To Adults Just Met & -0.58 \\
\hline & 106 & Acts Shy Around New People & -0.75 \\
\hline & 119 & Is Comfortable Asking Other Children To Play & 0.65 \\
\hline & 129 & Talks Easily To New People & 0.85 \\
\hline & 143 & Turns Away Shyly From New People & -0.68 \\
\hline & 158 & At Ease With Any Group & 0.82 \\
\hline
\end{tabular}




\begin{tabular}{|r|r|l|r|}
\hline Smiling & & & \\
\hline & 11 & Laughs At Jokes & 0.55 \\
\hline & 43 & Enjoys Funny Stories Doesn't Laugh At Them & -0.48 \\
\hline & 56 & Laughs During Play With Parents & 0.33 \\
\hline & 83 & Serious Expression Even During Play & -0.31 \\
\hline & 99 & Seldom Laugh Loud During Play & -0.54 \\
\hline & 110 & Smiles Giggles Playing By Self & 0.48 \\
\hline & 121 & Rarely Smiles When Playing With Pets & -0.34 \\
\hline & 135 & Doesn't Giggle Or Act Silly & -0.58 \\
\hline & 152 & Smiles At People Who Likes & 0.61 \\
\hline & 163 & Laughs Out Loud In Play With Children & 0.65 \\
\hline & 165 & Rarely Laughs Aloud When Watching Comedies & -0.50 \\
\hline & 179 & Smiles At Friendly Strangers & 0.32 \\
\hline & 194 & Smiles When Looking At Picture Book & 0.51 \\
\hline
\end{tabular}

Note. The table shows a CFA solution of the 15 lower-order scales plus Attentional Shifting (experimental scale) originally developed by Rothbart and colleagues (2001); Activity = Activity Level; Anger = Anger/Frustration; Approach = Approach/Positive Anticipation; AttnFocus = Attentional Focusing; AttnShift = Attentional Shifting; Sooth = Soothability/Falling Reactivity; HighPL = High Intensity Pleasure; InhibCn = Inhibitory Control; LowPL = Low Intensity Pleasure; PerSen = Perceptual Sensitivity; Smiling = Smiling/Laughter 
Table B

Lower-Order CFA Results for the Original CBQ Scales at Age 5/6

\begin{tabular}{|c|c|c|c|}
\hline Scale & Item \# & Abbreviated Item Description & $\begin{array}{r}\text { Standardized } \\
\text { Loadings }\end{array}$ \\
\hline \multicolumn{4}{|c|}{ Activity } \\
\hline & 1 & Seems Always In A Hurry & 0.03 \\
\hline & 25 & Runs Vs Walks & 0.11 \\
\hline & 41 & Sits Quietly Outside & 0.12 \\
\hline & 48 & Climbs In House & 0.69 \\
\hline & 88 & Sits Quietly For Long Periods & 0.21 \\
\hline & 102 & Prefers Quiet Activities Vs Games & -0.12 \\
\hline & 123 & Rarely Runs Moves Quickly In House & 0.33 \\
\hline & 126 & Plays Games Slowly Deliberately & -0.45 \\
\hline & 145 & Sits Quietly In Bath & -0.40 \\
\hline & 153 & Plays Actively Outdoors With Children & -0.24 \\
\hline & 172 & Full Of Energy In The Evening & 0.65 \\
\hline & 187 & Difficulty Sitting Still At Dinner & -0.11 \\
\hline & 192 & Likes To Sit Quietly and Watch People Do Things & -0.09 \\
\hline \multicolumn{4}{|c|}{ 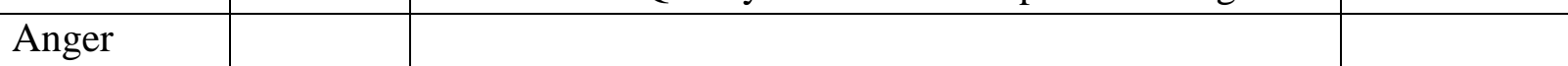 } \\
\hline & 2 & Angry When Asked to Go to Bed & 0.12 \\
\hline & 19 & Rarely Irritated When Makes Mistake & -0.09 \\
\hline & 34 & Has Temper Tantrums & -0.16 \\
\hline & 62 & Frustrated When Prevented From Doing Things & -0.22 \\
\hline & 73 & Gets Mad When Mildly Criticized & 0.19 \\
\hline & 78 & Angry When Can't Find Smth. Wants To Play With & 0.54 \\
\hline & 120 & Rarely Gets Upset When Told To Go To Bed & 0.55 \\
\hline & 128 & Easily Frustrated When Tired & -0.32 \\
\hline & 140 & Irritable When Asked To Eat Food Doesn't Like & 0.31 \\
\hline & 156 & Rarely Protests When Another Child Takes Toy & 0.04 \\
\hline & 173 & Easily Irritated When Trouble With Task & 0.25 \\
\hline & 181 & Angry When Called In From Play & 0.15 \\
\hline & 193 & Gets Mad When Provoked & -0.30 \\
\hline \multicolumn{4}{|c|}{ Approach } \\
\hline & 10 & Gets Worked Up \& Has Trouble Sitting Still & -0.01 \\
\hline & 24 & Excited About Getting Toy & -0.15 \\
\hline & 35 & Wants To Do Smth. Talks About Little Else & 0.56 \\
\hline & 69 & Strong Desires For Certain Foods & 0.50 \\
\hline & 82 & Looks Forward To Visits Of Loved Relatives & 0.19 \\
\hline & 96 & Excited When Planning Trips & 0.60 \\
\hline
\end{tabular}




\begin{tabular}{|c|c|c|c|}
\hline & 117 & Excited Before Outing & -0.37 \\
\hline & 131 & Calm Before Outing & 0.21 \\
\hline & 148 & Gets Enthusiastic About Things & -0.29 \\
\hline & 166 & Excited When Opens Presents & 0.17 \\
\hline & 175 & Is Not Excited About TV Programs & -0.22 \\
\hline & 188 & Remains Calm About Desserts & 0.47 \\
\hline & 191 & Looks Forward Family Outings But Not Excited & -0.14 \\
\hline \multicolumn{4}{|l|}{ AttnFocus } \\
\hline & 16 & Keeps At Task Until Done & -0.17 \\
\hline & 38 & Has A Hard Time Keeping Mind On Activity & 0.56 \\
\hline & 47 & $\begin{array}{l}\text { Moves From One Task To Another Without } \\
\text { Completing }\end{array}$ & -0.45 \\
\hline & 125 & Good Concentration When Drawing & -0.42 \\
\hline & 144 & When Becomes Involved Works For Long Periods & 0.68 \\
\hline & 160 & Difficulty Leaving Project & 0.26 \\
\hline & 171 & Easily Distracted When Listening To Story & 0.03 \\
\hline & 186 & Absorbed In Picture Book & 0.06 \\
\hline & 195 & Trouble Concentrating When Distracting Noises & -0.17 \\
\hline \multicolumn{4}{|l|}{ AttnShift } \\
\hline & 6 & Hard To Get Attention When Concentrating & -0.08 \\
\hline & 29 & Shifts Easily From One Activity To Another & 0.08 \\
\hline & 95 & Trouble Stopping Activity & 0.15 \\
\hline & 180 & Easy Time Leaving Play To Come To Dinner & -0.68 \\
\hline & 184 & Doesn't Seem To Hear When Talked To & 0.80 \\
\hline \multicolumn{4}{|l|}{ Discomfort } \\
\hline & 5 & Not Very Bothered By Pain & 0.39 \\
\hline & 21 & Uncomfortable Cold Wet & 0.32 \\
\hline & 61 & Upset By A Little Cut Bruise & 0.05 \\
\hline & 87 & Bothered By Light Or Bright Colors & -0.52 \\
\hline & 97 & Finds Rough Materials Uncomfortable & 0.06 \\
\hline & 101 & Not Upset By Cuts Bruises & -0.02 \\
\hline & 115 & Bothered By Bath Too Hot Cold & -0.39 \\
\hline & 132 & Likely To Cry When A Bit Hurt & 0.29 \\
\hline & 141 & Distressed When Hair Combed & -0.32 \\
\hline & 157 & Cries When Given Injection & 0.26 \\
\hline & 178 & Bothered By Loud Sounds & 0.32 \\
\hline & 190 & Seldom Complains When Sick & -0.16 \\
\hline \multicolumn{4}{|l|}{ Soothability } \\
\hline & 14 & Hard Time Settling For A Nap & 0.59 \\
\hline & 27 & Calms Quickly After Exciting Event & 0.43 \\
\hline & 42 & Can Be Cheered Up By Talking About Smth. & -0.24 \\
\hline
\end{tabular}




\begin{tabular}{|c|c|c|c|}
\hline & & Interesting & \\
\hline & 53 & Hard Time Settling Down After Exciting Activity & -0.19 \\
\hline & 68 & When Angry Upset For 10 Min. Or Longer & 0.19 \\
\hline & 85 & Forgets Bumps Scrapes After Couple Min. & -0.07 \\
\hline & 92 & Changes Feelings Fast & 0.03 \\
\hline & 103 & Falls Asleep In 10 Min. & -0.40 \\
\hline & 118 & Cheers Quickly If Upset & 0.14 \\
\hline & 134 & Easy To Soothe & -0.17 \\
\hline & 150 & Difficult To Soothe When Upset & -0.23 \\
\hline & 167 & Hard Time Going Back To Sleep After Waking & 0.12 \\
\hline & 177 & Doesn't Cry For More Than Couple Minutes & -0.04 \\
\hline \multicolumn{4}{|l|}{ Fear } \\
\hline & 15 & Not Afraid Of Large Dogs & -0.05 \\
\hline & 40 & Afraid Of Burglars Boogie Man & 0.12 \\
\hline & 50 & Afraid Of Loud Noises & 0.07 \\
\hline & 58 & Doesn't Worry About Injections & -0.77 \\
\hline & 70 & Not Afraid Of Dark & 0.07 \\
\hline & 80 & Afraid Of Fire & 0.20 \\
\hline & 91 & Frightened By Nightmares & 0.26 \\
\hline & 130 & Afraid Of The Dark & -0.86 \\
\hline & 138 & Rarely Frightened By Monsters On TV & 0.20 \\
\hline & 161 & Not Afraid Of Heights & 0.07 \\
\hline & 176 & Not Afraid To Sleep Alone & 0.08 \\
\hline & 189 & Nervous About Dentist & 0.09 \\
\hline \multicolumn{4}{|l|}{ HighPL } \\
\hline & 8 & Adventurous & 0.51 \\
\hline & 22 & Plays Wild Recklessly & 0.10 \\
\hline & 30 & Does Not Care For Rough Games & -0.12 \\
\hline & 51 & Does Not Like Taking Chances & 0.20 \\
\hline & 60 & Does Not Like High Slides & -0.61 \\
\hline & 67 & Enjoys Being Chased Spun & 0.05 \\
\hline & 77 & Enjoys Crowds of People & 0.02 \\
\hline & 100 & Enjoys Exciting Suspenseful TV & 0.20 \\
\hline & 107 & Enjoys Santa Other Strangers In Costumes & 0.79 \\
\hline & 124 & Enjoys Exploring New Places & 0.09 \\
\hline & 139 & Likes To Go High Fast When On Swing & -0.17 \\
\hline & 159 & Dislikes Rowdy Games & -0.75 \\
\hline & 182 & Enjoys Riding Bike Fast Recklessly & 0.03 \\
\hline \multicolumn{4}{|l|}{ Impulsivity } \\
\hline & 13 & Rushes Into Activity Without Thinking & -0.21 \\
\hline
\end{tabular}




\begin{tabular}{|c|c|c|c|}
\hline & 26 & Interrupts Others & -0.52 \\
\hline & 46 & Goes After What He/She Wants & -0.03 \\
\hline & 59 & Rushes Into New Situations & 0.15 \\
\hline & 71 & Long Time In Approaching New Situations & 0.16 \\
\hline & 79 & Stops Thinks Before Doing & -0.46 \\
\hline & 90 & Slow At Deciding What To Do & -0.04 \\
\hline & 104 & Says What Comes to Mind & 0.20 \\
\hline & 114 & When Eager To Go Outside/Rushes & 0.34 \\
\hline & 137 & Approaches Slowly Places Where Might Get Hurt & 0.59 \\
\hline & 155 & Eager To Have A Toy Game Right Then & -0.01 \\
\hline & 169 & Does Not Try New Activity & 0.60 \\
\hline & 183 & Slow To Warm To Others & -0.38 \\
\hline \multicolumn{4}{|l|}{ InhibCn } \\
\hline & 4 & Can Lower Voice When Asked & 0.04 \\
\hline & 20 & Good At Simon Says & -0.25 \\
\hline & 32 & Hard Time Following Instructions & -0.15 \\
\hline & 63 & Plans For Trips Outings & 0.51 \\
\hline & 75 & Can Wait When Asked To & 0.30 \\
\hline & 93 & Difficulty Waiting In Line & -0.23 \\
\hline & 108 & Trouble Sitting Still When Told & -0.15 \\
\hline & 116 & Able To Resist Inappropriate Laughing & 0.15 \\
\hline & 136 & Good At Following Instructions & 0.07 \\
\hline & 147 & Cautiously Approaches Dangerous Places & -0.21 \\
\hline & 162 & Not Careful In Crossing Streets & -0.11 \\
\hline & 168 & Can Easily Stop When Told "No" & 0.30 \\
\hline & 185 & Able To Resist Temptation & 0.38 \\
\hline \multicolumn{4}{|l|}{ LowPL } \\
\hline & 12 & Does Not Enjoy Being Talked To & 0.00 \\
\hline & 36 & Enjoys Sitting In Sunshine & 0.43 \\
\hline & 54 & Enjoys Baths & 0.56 \\
\hline & 66 & Doesn't Like Being Read To & -0.18 \\
\hline & 76 & Enjoys Snuggle To Parent Or Babysitter & -0.25 \\
\hline & 86 & Doesn't Like Quiet Games & -0.23 \\
\hline & 111 & Is Not Interested In Quiet TV & -0.14 \\
\hline & 113 & Enjoys Being Talked To & 0.12 \\
\hline & 133 & Enjoys Picture Books & 0.21 \\
\hline & 146 & Likes Being Sung To & -0.19 \\
\hline & 151 & Likes Sounds Of Words Nursery Rhymes & 0.48 \\
\hline & 164 & Enjoys Rhythmic Activities As Rocking & -0.22 \\
\hline & 174 & Enjoys Sitting On Parents Lap & 0.55 \\
\hline
\end{tabular}




\begin{tabular}{|c|c|c|c|}
\hline PerSen & & & \\
\hline & 9 & Notices Smoothness Roughness & -0.52 \\
\hline & 28 & Does Not Comment On Changes In Appearance & -0.04 \\
\hline & 31 & Notices New Clothing & 0.45 \\
\hline & 52 & Listens To Quiet Sounds & 0.62 \\
\hline & 65 & Comments On Changes In Parent Appearance & 0.00 \\
\hline & 84 & Doesn't Comment On Facial Features & 0.34 \\
\hline & 98 & Quickly Aware Of New Item In Living Room & 0.21 \\
\hline & 105 & Comments On Unusual Voice & -0.08 \\
\hline & 122 & Does Not Notice Parents' Facial Expressions & 0.23 \\
\hline & 142 & Doesn't React To Food Textures & 0.23 \\
\hline & 154 & Notices Specks of Dirt & -0.42 \\
\hline & 170 & Doesn't Notice Odors Perfume Smoke Cooking & 0.69 \\
\hline Sadness & & & \\
\hline & 18 & Cries Sadly When Toy Broken & -0.17 \\
\hline & 39 & Feels Down At The End Of An Exciting Day & 0.63 \\
\hline & 44 & Becomes Sad When Plans Don't Work Out & 0.20 \\
\hline & 55 & Depressed When Cannot Complete Task & -0.17 \\
\hline & 64 & Gets Upset When Relatives Leave & -0.34 \\
\hline & 72 & Doesn't Become Tearful When Tired & 0.14 \\
\hline & 81 & Feelings Easily Hurt By What Parents Say & 0.08 \\
\hline & 94 & $\begin{array}{l}\text { Tearful When Told To Do Smth. Does Not Want To } \\
\text { Do }\end{array}$ & 0.61 \\
\hline & 109 & Rarely Cries When Hears Sad Story & 0.65 \\
\hline & 112 & Rarely Upset When Watching Sad TV & 0.31 \\
\hline & 127 & Appears Downcast For No Reason & -0.41 \\
\hline & 149 & Rarely Discouraged When Trouble Doing Smth. & -0.18 \\
\hline Shyness & & & \\
\hline & 7 & Prefers To Watch Vs. Join Playing & 0.39 \\
\hline & 17 & Comfortable Meet Others & -0.41 \\
\hline & 23 & At Ease With Anyone & 0.50 \\
\hline & 37 & Gets Embarrassed When Strangers Pay Attention & -0.32 \\
\hline & 45 & Acts Friendly With New Children & 0.30 \\
\hline & 57 & Joins Others Quickly Even If Strangers & -0.16 \\
\hline & 74 & Shy Even With People Who Knows For Long Time & 0.42 \\
\hline & 89 & Nervous When Talking To Adults Just Met & -0.09 \\
\hline & 106 & Acts Shy Around New People & 0.08 \\
\hline & 119 & Is Comfortable Asking Other Children To Play & -0.32 \\
\hline & 129 & Talks Easily To New People & 0.38 \\
\hline & 143 & Turns Away Shyly From New People & -0.10 \\
\hline & 158 & At Ease With Any Group & 0.13 \\
\hline
\end{tabular}




\begin{tabular}{|r|r|l|r|}
\hline Smiling & & & \\
\hline & 11 & Laughs At Jokes & 0.48 \\
\hline & 43 & Enjoys Funny Stories Doesn't Laugh At Them & -0.24 \\
\hline & 56 & Laughs During Play With Parents & 0.40 \\
\hline & 83 & Serious Expression Even During Play & -0.14 \\
\hline & 99 & Seldom Laugh Loud During Play & -0.11 \\
\hline & 110 & Smiles Giggles Playing By Self & 0.10 \\
\hline & 121 & Rarely Smiles When Playing With Pets & -0.36 \\
\hline & 135 & Doesn't Giggle Or Act Silly & -0.51 \\
\hline & 152 & Smiles At People Who Likes & -0.21 \\
\hline & 163 & Laughs Out Loud In Play With Children & 0.28 \\
\hline & 165 & Rarely Laughs Aloud When Watching Comedies & -0.01 \\
\hline & 179 & Smiles At Friendly Strangers & 0.29 \\
\hline & 194 & Smiles When Looking At Picture Book & 0.32 \\
\hline
\end{tabular}

Note. The table shows a CFA solution of the 15 lower-order scales plus Attentional Shifting (experimental scale) originally developed by Rothbart and colleagues (2001); Activity = Activity Level; Anger = Anger/Frustration; Approach = Approach/Positive Anticipation; AttnFocus = Attentional Focusing; AttnShift = Attentional Shifting; Sooth = Soothability/Falling Reactivity; HighPL = High Intensity Pleasure; InhibCn = Inhibitory Control; LowPL = Low Intensity Pleasure; PerSen = Perceptual Sensitivity; Smiling = Smiling/Laughter 
Table C

CFA solution for the Three Higher-Order CBQ Factors reported by Rothbart et al. (2001)

\begin{tabular}{|l|l|l|l|}
\hline Factor & Scale & Standardized Loadings (3 y.o.) & Standardized Loadings (5/6 y.o.) \\
\hline Surgency & & & \\
\hline & Activity & 0.70 & 0.66 \\
\hline & HighPL & 0.63 & 0.64 \\
\hline & Impulsivity & 0.91 & 0.89 \\
\hline & Shyness & -0.58 & -0.59 \\
\hline & Smiling & 0.70 & 0.72 \\
\hline & Approach & 0.53 & 0.46 \\
\hline NA & & & \\
\hline & Anger & 0.76 & 0.78 \\
\hline & Discomfort & 0.56 & 0.59 \\
\hline & Fear & 0.46 & 0.50 \\
\hline & Sadness & 0.64 & 0.68 \\
\hline & Soothability & -0.66 & -0.70 \\
\hline EC & & & \\
\hline & AttnFocus & 0.64 & 0.68 \\
\hline & InhibCn & 0.79 & 0.86 \\
\hline & LowPL & 0.67 & 0.62 \\
\hline & PerSen & 0.40 & 0.26 \\
\hline & Smiling & 0.64 & 0.69 \\
\hline & Approach & 0.21 & 0.07 \\
\hline
\end{tabular}

Note. The table depicts a CFA solution of the three higher-order factors originally reported by Rothbart and colleagues (2001); NA = Negative Affectivity; EC = Effortful Control; Activity = Activity Level; Anger = Anger/Frustration; Approach = Approach/Positive Anticipation;

AttnFocus = Attentional Focusing; AttnShift = Attentional Shifting; Sooth = Soothability $/$ Falling Reactivity; HighPL = High Intensity Pleasure; InhibCn = Inhibitory Control; LowPL = Low Intensity Pleasure; PerSen = Perceptual Sensitivity; Smiling = Smiling/Laughter; Approach/Positive Anticipation and Smiling/Laughter were set to load on Surgency and EC as per the solution reported by Rothbart and colleagues (2001). 
Table D

Items Excluded from Higher-Order EFA Analyses at Age 3 Due to Low Loadings

\begin{tabular}{|c|c|c|}
\hline Item\# & Original CBQ Scale & Abbreviated Item Description \\
\hline 1 & Activity Level & Seems Always In A Hurry \\
\hline 123 & Activity Level & Rarely Runs Or Moves Quickly In House \\
\hline 145 & Activity Level & Sits Quietly In Bath \\
\hline 187 & Activity Level & Difficulty Sitting Still At Dinner \\
\hline 73 & Anger/Frustration & Gets Mad When Mildly Criticized \\
\hline 140 & Anger/Frustration & Irritable When Asked To Eat Food Doesn't Like \\
\hline 156 & Anger/Frustration & Rarely Protests When Another Child Takes Toy \\
\hline 181 & Anger/Frustration & Angry When Called In From Play \\
\hline 10 & Approach/Positive Anticipation & Gets Worked Up \& Has Trouble Sitting Still \\
\hline 24 & Approach/Positive Anticipation & Excited About Getting Toy \\
\hline 35 & Approach/Positive Anticipation & When Wants To Do Smth., Talks About Little Else \\
\hline 69 & Approach/Positive Anticipation & Strong Desires For Certain Foods \\
\hline 82 & Approach/Positive Anticipation & Looks Forward To Visits Of Loved Relatives \\
\hline 148 & Approach/Positive Anticipation & Gets Enthusiastic About Things \\
\hline 166 & Approach/Positive Anticipation & Excited When Opens Presents \\
\hline 175 & Approach/Positive Anticipation & Is Not Excited About TV Programs \\
\hline 188 & Approach/Positive Anticipation & Remains Calm About Desserts \\
\hline 16 & Attentional Focusing & Keeps At Task Until Done \\
\hline 160 & Attentional Focusing & Difficulty Leaving Project \\
\hline 186 & Attentional Focusing & Absorbed In Picture Books \\
\hline 6 & Attentional Shifting & Hard To Get Attention When Concentrating \\
\hline 180 & Attentional Shifting & Easy Time Leaving Play To Come To Dinner \\
\hline 184 & Attentional Shifting & Doesn't Seem To Hear When Talked To \\
\hline 5 & Discomfort & Not Very Bothered By Pain \\
\hline 21 & Discomfort & Uncomfortable When Cold/Wet \\
\hline 87 & Discomfort & Bothered By Light Or Bright Color \\
\hline 97 & Discomfort & Finds Rough Materials Uncomfortable \\
\hline 115 & Discomfort & Bothered By Bath/Too Hot, Cold \\
\hline 141 & Discomfort & Distressed When Hair Combed \\
\hline 157 & Discomfort & Cries When Given Injections \\
\hline 190 & Discomfort & Seldom Complains When Sick \\
\hline 15 & Fear & Not Afraid Of Large Dogs \\
\hline 40 & Fear & Afraid Of Burglars/Boogie Man \\
\hline 58 & Fear & Doesn't Worry About Injections \\
\hline 80 & Fear & Afraid Of Fire \\
\hline 91 & Fear & Frightened By Nightmares \\
\hline 138 & Fear & Rarely Frightened By Monsters On TV \\
\hline
\end{tabular}




\begin{tabular}{|c|c|c|}
\hline 176 & Fear & Not Afraid To Sleep Alone \\
\hline 189 & Fear & Nervous About Dentist \\
\hline 67 & High Intensity Pleasure & Enjoys Being Chased/Spun \\
\hline 100 & High Intensity Pleasure & Enjoys Exciting Suspenseful TV \\
\hline 107 & High Intensity Pleasure & Enjoys Santa/Other Strangers In Costumes \\
\hline 124 & High Intensity Pleasure & Enjoys Exploring New Places \\
\hline 182 & High Intensity Pleasure & Enjoys Riding Bike Fast/Recklessly \\
\hline 13 & Impulsivity & Rushes Into Activity Without Thinking \\
\hline 59 & Impulsivity & Rushes Into New Situations \\
\hline 79 & Impulsivity & Stops and Thinks Before Doing \\
\hline 90 & Impulsivity & Slow At Deciding What To Do \\
\hline 104 & Impulsivity & Says What Comes to Mind \\
\hline 114 & Impulsivity & When Eager To Go Outside Rushes \\
\hline 137 & Impulsivity & Approaches Slowly Places Where Might Get Hurt \\
\hline 155 & Impulsivity & Eager To Have A Toy Game Right Then \\
\hline 169 & Impulsivity & Does Not Try New Activity \\
\hline 4 & Inhibitory Control & Can Lower Voice When Asked \\
\hline 20 & Inhibitory Control & Good At Simon Says \\
\hline 63 & Inhibitory Control & Plans For Trips/Outings \\
\hline 75 & Inhibitory Control & Can Wait When Asked To \\
\hline 93 & Inhibitory Control & Difficulty Waiting In Line \\
\hline 108 & Inhibitory Control & Trouble Sitting Still When Told \\
\hline 116 & Inhibitory Control & Able To Resist Inappropriate Laughing \\
\hline 136 & Inhibitory Control & Good At Following Instructions \\
\hline 147 & Inhibitory Control & Cautiously Approaches Dangerous Places \\
\hline 162 & Inhibitory Control & Not Careful In Crossing Streets \\
\hline 12 & Low Intensity Pleasure & Does Not Enjoy Being Talked To \\
\hline 54 & Low Intensity Pleasure & Enjoys Baths \\
\hline 66 & Low Intensity Pleasure & Doesn't Like Being Read To \\
\hline 76 & Low Intensity Pleasure & Enjoys Snuggling To Parent Or Babysitter \\
\hline 86 & Low Intensity Pleasure & Doesn't Like Quiet Games \\
\hline 111 & Low Intensity Pleasure & Is Not Interested In Quiet TV \\
\hline 113 & Low Intensity Pleasure & Enjoys Being Talked To \\
\hline 133 & Low Intensity Pleasure & Enjoys Picture Books \\
\hline 146 & Low Intensity Pleasure & Likes Being Sung To \\
\hline 164 & Low Intensity Pleasure & Enjoys Rhythmic Activities As Rocking \\
\hline 174 & Low Intensity Pleasure & Enjoys Sitting On Parent's Lap \\
\hline 9 & Perceptual Sensitivity & Notices Smoothness/Roughness \\
\hline 52 & Perceptual Sensitivity & Listens To Quiet Sounds \\
\hline 84 & Perceptual Sensitivity & Doesn't Comment On Facial Features \\
\hline 98 & Perceptual Sensitivity & Quickly Aware Of New Item In Living Room \\
\hline 105 & Perceptual Sensitivity & Comments On Unusual Voice \\
\hline
\end{tabular}




\begin{tabular}{|c|c|c|}
\hline 122 & Perceptual Sensitivity & Does Not Notice Parent's Facial Expressions \\
\hline 142 & Perceptual Sensitivity & Doesn't React To Food Textures \\
\hline 154 & Perceptual Sensitivity & Notices Specks of Dirt \\
\hline 170 & Perceptual Sensitivity & Doesn't Notice Odors/Perfume/Smoke/Cooking \\
\hline 18 & Sadness & Cries Sadly When Toy Broken \\
\hline 39 & Sadness & Feels Down At The End Of An Exciting Day \\
\hline 44 & Sadness & Becomes Sad When Plans Don't Work Out \\
\hline 64 & Sadness & Gets Upset When Relatives Leave \\
\hline 72 & Sadness & Doesn't Become Tearful When Tired \\
\hline 81 & Sadness & Feelings Easily Hurt By What Parents Say \\
\hline 94 & Sadness & $\begin{array}{l}\text { Tearful When Told To Do Smth Does Not Want To } \\
\text { Do }\end{array}$ \\
\hline 109 & Sadness & Rarely Cries When Hears Sad Story \\
\hline 112 & Sadness & Rarely Upset When Watching Sad TV \\
\hline 127 & Sadness & Appears Downcast For No Reason \\
\hline 7 & Shyness & Prefers To Watch Vs. Join Playing \\
\hline 43 & Smiling/Laughter & Enjoys Funny Stories/Doesn't Laugh At Them \\
\hline 56 & Smiling/Laughter & Laughs During Play With Parents \\
\hline 83 & Smiling/Laughter & Serious Expression Even During Play \\
\hline 121 & Smiling/Laughter & Rarely Smiles When Playing With Pets \\
\hline 194 & Smiling/Laughter & Smiles When Looking At Picture Books \\
\hline 14 & Soothability/Falling Reactivity & Hard Time Settling For A Nap \\
\hline 27 & Soothability/Falling Reactivity & Calms Quickly After Exciting Event \\
\hline 42 & Soothability/Falling Reactivity & $\begin{array}{l}\text { Can Be Cheered Up By Talking About Smth } \\
\text { Interesting }\end{array}$ \\
\hline 53 & Soothability/Falling Reactivity & Hard Time Settling Down After Exciting Activity \\
\hline 167 & Soothability/Falling Reactivity & Hard Time Going Back To Sleep After Waking \\
\hline 3 & No scale assigned & Feelings Not Easily Hurt \\
\hline 33 & No scale assigned & Afraid Of Elevators \\
\hline 49 & No scale assigned & Dislikes Having Nails Cut \\
\hline
\end{tabular}


Table E

Items Excluded from Higher-Order EFA Analyses at Age 5/6 Due to Low Loadings

\begin{tabular}{|c|c|c|}
\hline Item\# & Original CBQ Scale & Abbreviated Item Description \\
\hline 1 & Activity Level & Seems Always In A Hurry \\
\hline 25 & Activity Level & Runs Vs. Walks \\
\hline 48 & Activity Level & Climbs In House \\
\hline 123 & Activity Level & Rarely Run/ Moves Quickly In House \\
\hline 145 & Activity Level & Sits Quietly In Bath \\
\hline 172 & Activity Level & Full Of Energy In The Evening \\
\hline 187 & Activity Level & Difficulty Sitting Still At Dinner \\
\hline 73 & Anger/Frustration & Gets Mad When Mildly Criticized \\
\hline 120 & Anger/Frustration & Rarely Gets Upset When Told To Go To Bed \\
\hline 128 & Anger/Frustration & Easily Frustrated When Tired \\
\hline 140 & Anger/Frustration & Irritable When Asked To Eat Food Doesn't Like \\
\hline 156 & Anger/Frustration & Rarely Protests When Another Child Takes Toy \\
\hline 193 & Anger/Frustration & Gets Mad When Provoked \\
\hline 10 & Approach/Positive Anticipation & Gets Worked Up \& Has Trouble Sitting Still \\
\hline 24 & Approach/Positive Anticipation & Excited About Getting Toy \\
\hline 35 & Approach/Positive Anticipation & When Wants To Do Smth Talks About Little Else \\
\hline 69 & Approach/Positive Anticipation & Strong Desires For Certain Foods \\
\hline 82 & Approach/Positive Anticipation & Looks Forward To Visits Of Loved Relatives \\
\hline 148 & Approach/Positive Anticipation & Gets Enthusiastic About Things \\
\hline 175 & Approach/Positive Anticipation & Is Not Excited About TV Programs \\
\hline 188 & Approach/Positive Anticipation & Remains Calm About Desserts \\
\hline 160 & Attentional Focusing & Difficulty Leaving Project \\
\hline 6 & Attentional Shifting & Hard To Get Attention When Concentrating \\
\hline 29 & Attentional Shifting & Shifts Easily From One Activity To Another \\
\hline 180 & Attentional Shifting & Easy Time Leaving Play To Come To Dinner \\
\hline 21 & Discomfort & Uncomfortable When Cold/Wet \\
\hline 87 & Discomfort & Bothered By Light Or Bright Color \\
\hline 97 & Discomfort & Finds Rough Materials Uncomfortable \\
\hline 115 & Discomfort & Bothered By Baths Too Hot Cold \\
\hline 141 & Discomfort & Distressed When Hair Combed \\
\hline 157 & Discomfort & Cries When Given Injections \\
\hline 190 & Discomfort & Seldom Complains When Sick \\
\hline 15 & Fear & Not Afraid Of Large Dogs \\
\hline 40 & Fear & Afraid Of Burglars/Boogie Man \\
\hline 58 & Fear & Doesn't Worry About Injections \\
\hline 80 & Fear & Afraid Of Fire \\
\hline 91 & Fear & Frightened By Nightmares \\
\hline 138 & Fear & Rarely Frightened By Monsters On TV \\
\hline
\end{tabular}




\begin{tabular}{|c|c|c|}
\hline 176 & Fear & Not Afraid To Sleep Alone \\
\hline 189 & Fear & Nervous About Dentist \\
\hline 22 & High Intensity Pleasure & Plays Wild/Recklessly \\
\hline 67 & High Intensity Pleasure & Enjoys Being Chased/Spun \\
\hline 100 & High Intensity Pleasure & Enjoys Exciting Suspenseful TV \\
\hline 107 & High Intensity Pleasure & Enjoys Santa/Other Strangers In Costumes \\
\hline 124 & High Intensity Pleasure & Enjoys Exploring New Places \\
\hline 26 & Impulsivity & Interrupts Others \\
\hline 46 & Impulsivity & Goes After What He/She Wants \\
\hline 90 & Impulsivity & Slow At Deciding What To Do \\
\hline 114 & Impulsivity & When Eager To Go Outside/Rushes \\
\hline 137 & Impulsivity & Approaches Slowly Places Where Might Get Hurt \\
\hline 155 & Impulsivity & Eager To Have A Toy/Game Right Then \\
\hline 169 & Impulsivity & Does Not Try New Activity \\
\hline 4 & Inhibitory Control & Can Lower Voice When Asked \\
\hline 63 & Inhibitory Control & Plans For Trips Outings \\
\hline 75 & Inhibitory Control & Can Wait When Asked To \\
\hline 93 & Inhibitory Control & Difficulty Waiting In Line \\
\hline 116 & Inhibitory Control & Able To Resist Inappropriate Laughing \\
\hline 147 & Inhibitory Control & Cautiously Approaches Dangerous Places \\
\hline 162 & Inhibitory Control & Not Careful In Crossing Streets \\
\hline 168 & Inhibitory Control & Can Easily Stop When Told No \\
\hline 185 & Inhibitory Control & Able To Resist Temptation \\
\hline 12 & Low Intensity Pleasure & Does Not Enjoy Being Talked To \\
\hline 54 & Low Intensity Pleasure & Enjoys Baths \\
\hline 66 & Low Intensity Pleasure & Doesn't Like Being Read To \\
\hline 76 & Low Intensity Pleasure & Enjoys Snuggling To Parent Or Babysitter \\
\hline 86 & Low Intensity Pleasure & Doesn't Like Quiet Games \\
\hline 111 & Low Intensity Pleasure & Is Not Interested In Quiet TV \\
\hline 113 & Low Intensity Pleasure & \begin{tabular}{|l} 
Enjoys Being Talked To \\
\end{tabular} \\
\hline 133 & Low Intensity Pleasure & Enjoys Picture Books \\
\hline 146 & Low Intensity Pleasure & Likes Being Sung To \\
\hline 151 & Low Intensity Pleasure & Likes Sounds Of Words/Nursery Rhymes \\
\hline 164 & Low Intensity Pleasure & Enjoys Rhythmic Activities As Rocking \\
\hline 174 & Low Intensity Pleasure & Enjoys Sitting On Parent's Lap \\
\hline 9 & Perceptual Sensitivity & Notices Smoothness/Roughness \\
\hline 52 & Perceptual Sensitivity & \begin{tabular}{|l} 
Listens To Quiet Sounds \\
\end{tabular} \\
\hline 84 & Perceptual Sensitivity & Doesn't Comment On Facial Features \\
\hline 105 & Perceptual Sensitivity & Comments On Unusual Voice \\
\hline 122 & Perceptual Sensitivity & Does Not Notice Parent's Facial Expressions \\
\hline 142 & Perceptual Sensitivity & Doesn't React To Food Textures \\
\hline 154 & Perceptual Sensitivity & Notices Specks Dirt \\
\hline 170 & Perceptual Sensitivity & Doesn't Notice Odors/Perfume/Smoke/Cooking \\
\hline
\end{tabular}




\begin{tabular}{|l|l|l|}
\hline 18 & Sadness & Cries Sadly When Toy Broken \\
\hline 39 & Sadness & Feels Down At The End Of An Exciting Day \\
\hline 44 & Sadness & Becomes Sad When Plans Don't Work Out \\
\hline 55 & Sadness & Depressed When Cannot Complete Task \\
\hline 64 & Sadness & Gets Upset When Relatives Leave \\
\hline 72 & Sadness & Doesn't Become Tearful When Tired \\
\hline 81 & Sadness & Feelings Easily Hurt By What Parents Say \\
\hline 94 & Sadness & Tearful When Told To Do Smth Does Not Want To \\
& & Do \\
\hline 127 & Sadness & Appears Downcast For No Reason \\
\hline 149 & Sadness & Rarely Discouraged When Trouble Doing Smth \\
\hline 11 & Smiling/Laughter & Laughs At Jokes \\
\hline 56 & Smiling/Laughter & Laughs During Play With Parents \\
\hline 83 & Smiling/Laughter & Serious Expression Even During Play \\
\hline 110 & Smiling/Laughter & Smiles Giggles Playing By Self \\
\hline 121 & Smiling/Laughter & Rarely Smiles When Playing With Pets \\
\hline 194 & Smiling/Laughter & Smiles When Looking At Picture Books \\
\hline 14 & Soothability/Falling Reactivity & Hard Time Settling For A Nap \\
\hline 27 & Soothability/Falling Reactivity & Calms Quickly After Exciting Event \\
\hline 42 & Soothability/Falling Reactivity & $\begin{array}{l}\text { Can Be Cheered Up By Talking About Smth } \\
\text { Interesting }\end{array}$ \\
\hline 53 & Soothability/Falling Reactivity & Hard Time Settling Down After Exciting Activity \\
\hline 68 & Soothability/Falling Reactivity & When Angry, Upset For 10 Min Or Longer \\
\hline 103 & Soothability/Falling Reactivity & Falls Asleep In 10 Min \\
\hline 167 & Soothability/Falling Reactivity & Hard Time Going Back To Sleep After Waking \\
\hline 177 & Soothability/Falling Reactivity & Doesn't Cry For More Than Couple Minutes \\
\hline 3 & No scale assigned & Feelings Not Easily Hurt \\
\hline 33 & No scale assigned & Afraid Of Elevators \\
\hline 49 & No scale assigned & Dislikes Having Nails Cut \\
\hline & & \\
\hline 14 & & \\
\hline & & \\
\hline
\end{tabular}


Table F

Higher-Order Exploratory Factor Analysis of the CBQ Lower-Order Scales Extracted at Age 3: Three-Factor Solution

\begin{tabular}{lrrr}
\hline Scales & Factor1 & Factor2 & Factor3 \\
\hline HighPL & 0.24 & 0.24 & $\mathbf{0 . 3 9}$ \\
(Low) Shy & 0.21 & $\mathbf{0 . 3 6}$ & 0.29 \\
Impulsivity & $\mathbf{0 . 5 7}$ & -0.02 & $\mathbf{0 . 4 9}$ \\
NoticeAp & $\mathbf{- 0 . 3 6}$ & -0.01 & 0.27 \\
(Low)AttnFocus & $\mathbf{0 . 5 6}$ & -0.23 & -0.03 \\
Ang/Sad & 0.17 & $-\mathbf{0 . 5 6}$ & 0.17 \\
AngAbtBed & 0.24 & $-\mathbf{0 . 3 5}$ & 0.02 \\
(Low) Activity & $-\mathbf{0 . 5 0}$ & -0.07 & -0.26 \\
Smiling & -0.04 & 0.27 & $\mathbf{0 . 6 0}$ \\
NotUpsetWPain & 0.28 & $\mathbf{0 . 5 2}$ & -0.03 \\
FearDark & -0.03 & -0.23 & -0.02 \\
InhibCn/AttnShift & $-\mathbf{0 . 5 7}$ & $\mathbf{0 . 4 2}$ & 0.00 \\
Approach & 0.01 & -0.09 & $\mathbf{0 . 5 1}$ \\
Soothability & 0.00 & $\mathbf{0 . 7 4}$ & 0.12 \\
FearOfLoudNoise & -0.08 & $\mathbf{- 0 . 6 3}$ & -0.05 \\
\hline
\end{tabular}

Note. Primary loadings of $\geq .40$ are bolded; loadings of $\geq .30$ are bolded and italicized; HighPL $=$ High Intensity Pleasure, LowShy $=$ low Shyness, Imp = Impulsivity; NoticeAp = Notices Appearances; LowAttnFoc = low Attentional Focusing; Ang/Sad = Anger and Sadness; AngAbtBed = Anger about going to bed; LowActiv = low Activity Level; Smiling = Smiling/Laughter; NotUpsetWithPain = Not upset by physical pain and bruises; FearDark = Fear of darkness; InhibCn/AttnShift = Inhibitory Control/Attentional Shifting; Approach = Approach/Positive Anticipation; Soothability = Soothability/Falling Reactivity; FearofLoudNoises = Fear of loud noises; In a three-factor solution, correlations between Factor 1 and 2 and 3 were -. 15 and .35 respectively; the correlation between Factor 2 and 3 was .20. 
Table G

Tests of Structural Invariance across the Two Samples (ON and NY)

\begin{tabular}{|c|c|c|c|c|c|c|}
\hline Model Tested & $d f$ & RMSEA & $\Delta$ RMSEA & CFI & $\Delta \mathrm{CFI}$ & PASS? \\
\hline \multicolumn{7}{|c|}{3 factors: 3-year-olds } \\
\hline Configural invariance & 130 & .065 & & .906 & & \\
\hline Weak invariance & 167 & .059 & .006 & .899 & .007 & YES \\
\hline Strong invariance & 182 & .061 & .002 & .882 & .017 & YES \\
\hline Strict invariance & 197 & .063 & .002 & .865 & .017 & YES \\
\hline \multicolumn{7}{|c|}{4 factors: 3-year-olds } \\
\hline Configural invariance & 142 & .056 & & .923 & & \\
\hline Weak invariance & 170 & .054 & .002 & .915 & .008 & YES \\
\hline Strong invariance & 185 & .057 & .003 & .898 & .017 & YES \\
\hline Strict invariance & 200 & .058 & .001 & .882 & .016 & YES \\
\hline \multicolumn{7}{|c|}{5 factors: 3-year olds } \\
\hline Configural invariance & 110 & .047 & & .957 & & \\
\hline Weak invariance & 150 & .045 & .002 & .948 & .009 & YES \\
\hline Strong invariance & 165 & .049 & .004 & .932 & .016 & YES \\
\hline Strict invariance & 180 & .051 & .002 & .919 & .013 & YES \\
\hline \multicolumn{7}{|c|}{3 factors: 6-year-olds } \\
\hline Configural invariance & 144 & .085 & & .812 & & \\
\hline Weak invariance & 174 & .077 & .008 & .814 & .002 & YES \\
\hline Strong invariance & 189 & .075 & .003 & .809 & .005 & YES \\
\hline Strict invariance & 204 & .073 & .002 & .806 & .003 & YES \\
\hline \multicolumn{7}{|c|}{4 factors: 6-year-olds 6} \\
\hline Configural invariance & 128 & .072 & & .880 & & \\
\hline Weak invariance & 164 & .064 & .008 & .879 & .001 & YES \\
\hline Strong invariance & 179 & .063 & .001 & .874 & .005 & YES \\
\hline Strict invariance & 192 & .050 & .013 & .914 & .040 & $\mathrm{NO}$ \\
\hline
\end{tabular}

${ }^{6}$ Residual variance parameter for Notices Appearances was restricted to 0 in both samples for model estimation purposes. 
Table H

Higher-Order Exploratory Factor Analysis of the CBQ Lower-Order Scales Extracted at Age 5/6: Three-Factor Solution

\begin{tabular}{lrrr}
\hline Scales & Factor1 & Factor2 & Factor3 \\
\hline Anger & $\mathbf{0 . 7 0}$ & 0.19 & 0.06 \\
(Low) EC & $\mathbf{0 . 5 0}$ & $\mathbf{0 . 4 7}$ & -0.02 \\
Sociability & -0.19 & 0.29 & $\mathbf{0 . 3 8}$ \\
NoticeAp & 0.02 & $\mathbf{- 0 . 3 6}$ & $\mathbf{0 . 4 2}$ \\
Advent & -0.16 & $\mathbf{0 . 4 2}$ & $\mathbf{0 . 3 5}$ \\
QuietP & -0.02 & $\mathbf{- 0 . 5 9}$ & $-\mathbf{0 . 3 3}$ \\
Smiling & -0.13 & -0.02 & $\mathbf{0 . 6 2}$ \\
NotUpsetWPain & $-\mathbf{0 . 4 6}$ & $\mathbf{0 . 3 8}$ & -0.02 \\
Approach & 0.23 & 0.00 & $\mathbf{0 . 4 7}$ \\
(Low) Activity & -0.13 & $-\mathbf{0 . 3 8}$ & -0.19 \\
IrritatedMistakes & $\mathbf{0 . 5 6}$ & 0.01 & 0.11 \\
FearDark & $\mathbf{0 . 3 0}$ & -0.17 & -0.03 \\
Soothability & $\mathbf{- 0 . 5 4}$ & -0.01 & $\mathbf{0 . 3 3}$ \\
FearOfLoudNoise & $\mathbf{0 . 6 2}$ & -0.14 & -0.21 \\
NotUpsetSadStories & $\mathbf{0 . 0 0}$ & 0.27 & -0.24 \\
\hline
\end{tabular}

Note. Primary loadings of $\geq .40$ are bolded; loadings of $\geq .30$ are bolded and italicized; Anger $=$ Anger/Frustration; LowEC $=$ low effortful control; NoticeAp = Notices Appearances; Advent = Adventurous; QuietP = Quiet Play; Smiling= Smiling/Laughter; NotUpsetWithPain = Not upset by physical pain and bruises; Approach = Approach/Positive Anticipation; (Low) Activity = low Activity Level; IrritatedMistakes = Irritated by mistakes; FearDark = Fear of darkness; Soothability = Soothability/Falling Reactivity; FearofLoudNoises = Fear of loud noises; NotUpsetSadStories = Not upset by sad stories; In a three-factor solution, correlations between Factor 1 and 2 and 3 were -.12 and .13 respectively; the correlation between Factor 2 and 3 was -.15. 
Table I

Higher-Order Exploratory Factor Analysis of the CBQ Lower-Order Scales Extracted at Age 5/6: Three-Factor Solutions in NY and ON Samples

\begin{tabular}{lrrrlrrr} 
NY Sample & \multicolumn{7}{c}{ ON Sample } \\
\hline Scales & Factor1 & Factor2 & Factor3 & Scale & Factor1 & Factor2 & Factor3 \\
\hline Anger & $\mathbf{0 . 6 3}$ & -0.25 & 0.02 & Anger & 0.25 & $\mathbf{0 . 7 1}$ & 0.00 \\
(Low) EC & $\mathbf{0 . 7 0}$ & -0.01 & -0.27 & (Low) EC & $\mathbf{0 . 4 9}$ & $\mathbf{0 . 5 2}$ & -0.01 \\
Sociability & 0.15 & $\mathbf{0 . 5 1}$ & 0.11 & Sociability & 0.29 & -0.02 & $\mathbf{0 . 3 9}$ \\
NoticeAp & -0.15 & -0.02 & $\mathbf{0 . 6 0}$ & NoticeAp & $\mathbf{- 0 . 3 6}$ & 0.02 & $\mathbf{0 . 3 6}$ \\
Advent & 0.29 & $\mathbf{0 . 5 3}$ & 0.03 & Advent & $\mathbf{0 . 4 7}$ & -0.06 & $\mathbf{0 . 3 0}$ \\
QuietP & $\mathbf{0 . 5 5}$ & $\mathbf{- 0 . 5 3}$ & 0.05 & QuietP & $\mathbf{- 0 . 6 8}$ & -0.11 & -0.23 \\
Smiling & 0.00 & $\mathbf{0 . 3 5}$ & $\mathbf{0 . 5 5}$ & Smiling & 0.03 & -0.01 & $\mathbf{0 . 5 7}$ \\
NotUpsetWPain & -0.02 & $\mathbf{0 . 4 8}$ & -0.27 & NotUpsetWPain & $\mathbf{0 . 3 8}$ & $\mathbf{- 0 . 4 5}$ & -0.02 \\
Approach & 0.25 & 0.14 & $\mathbf{0 . 4 3}$ & Approach & 0.01 & $\mathbf{0 . 3 6}$ & $\mathbf{0 . 4 4}$ \\
(Low) Activity & $-\mathbf{0 . 4 1}$ & -0.21 & -0.01 & (Low) Activity & $-\mathbf{0 . 4 8}$ & -0.14 & -0.10 \\
IrritatedMistakes & $\mathbf{0 . 4 5}$ & -0.21 & 0.11 & IrritatedMistakes & -0.03 & $\mathbf{0 . 5 9}$ & 0.09 \\
FearDark & $\mathbf{0 . 0 9}$ & -0.28 & 0.13 & FearDark & -0.17 & 0.22 & -0.08 \\
Soothability & $\mathbf{- 0 . 3 0}$ & $\mathbf{0 . 5 0}$ & 0.18 & Soothability & -0.12 & $\mathbf{- 0 . 4 0}$ & $\mathbf{0 . 4 5}$ \\
FearOfLoudNoise & 0.20 & $\mathbf{- 0 . 5 9}$ & 0.00 & FearOfLoudNoise & -0.03 & $\mathbf{0 . 5 8}$ & -0.25 \\
NotUpsetSadStories & 0.18 & 0.07 & $-\mathbf{0 . 3 6}$ & NotUpsetSadStories & 0.24 & -0.02 & -0.25 \\
\hline
\end{tabular}

Note. primary loadings of $\geq .40$ are bolded; loadings of $\geq .30$ are bolded and italicized; Anger $=$ Anger/Frustration; LowEC $=$ low effortful control; NoticeAp = Notices Appearances; Advent = Adventurous; QuietP = Quiet Play; Smiling= Smiling/Laughter; NotUpsetWithPain = Not upset by physical pain and bruises; Approach = Approach/Positive Anticipation; (Low) Activity = low Activity Level; IrritatedMistakes = Irritated by mistakes; FearDark = Fear of darkness; Soothability = Soothability/Falling Reactivity; FearofLoudNoises $=$ Fear of loud noises; NotUpsetSadStories $=$ Not upset by sad stories 
Table J

Higher-Order Exploratory Factor Analysis of the CBQ Lower-Order Scales Extracted at Age 5/6: Four-Factor Solutions in NY and ON Samples

\begin{tabular}{|c|c|c|c|c|c|c|c|c|c|}
\hline NY sample & & & & & ON sample & & & & \\
\hline Scales & Factor1 & Factor2 & Factor3 & Factor4 & Scale & Factor1 & Factor2 & Factor3 & Factor4 \\
\hline Anger & 0.58 & -0.01 & -0.04 & -0.30 & Anger & 0.34 & 0.683 & 0.01 & -0.047 \\
\hline (Low) EC & 0.78 & -0.39 & -0.01 & 0.00 & (Low) EC & 0.64 & 0.52 & 0.04 & 0.04 \\
\hline Sociability & 0.26 & 0.05 & 0.05 & 0.49 & Sociability & 0.09 & 0.04 & 0.39 & 0.25 \\
\hline NoticeAp & -0.08 & 0.60 & -0.09 & 0.02 & NoticeAp & -0.50 & 0.10 & 0.17 & 0.10 \\
\hline Advent & -0.01 & 0.14 & 0.67 & 0.00 & Advent & 0.01 & -0.16 & 0.68 & -0.02 \\
\hline QuietP & -0.29 & -0.01 & -0.60 & -0.02 & QuietP & -0.26 & 0.02 & -0.67 & 0.07 \\
\hline Smiling & 0.02 & 0.54 & 0.08 & 0.24 & Smiling & -0.23 & 0.04 & 0.43 & 0.22 \\
\hline NotUpsetWPain & -0.06 & -0.26 & 0.22 & 0.36 & NotUpsetWPain & 0.23 & -0.43 & 0.18 & 0.16 \\
\hline Approach & 0.21 & 0.43 & 0.11 & -0.03 & Approach & -0.08 & 0.39 & 0.26 & 0.14 \\
\hline (Low) Activity & -0.40 & 0.02 & -0.08 & -0.10 & (Low) Activity & -0.38 & -0.08 & -0.25 & 0.00 \\
\hline IrritatedMistakes & 0.36 & 0.12 & 0.01 & -0.30 & IrritatedMistakes & 0.06 & 0.58 & -0.03 & -0.05 \\
\hline FearDark & 0.20 & 0.09 & -0.23 & -0.12 & FearDark & 0.01 & 0.27 & -0.23 & 0.03 \\
\hline Soothability & 0.01 & 0.08 & -0.20 & 0.83 & Soothability & -0.04 & -0.03 & -0.05 & 0.95 \\
\hline FearOfLoudNoise & 0.03 & 0.07 & -0.02 & -0.71 & FearOfLoudNoise & -0.05 & 0.39 & -0.02 & -0.52 \\
\hline NotUpsetSadStories & 0.04 & -0.32 & 0.19 & -0.08 & NotUpsetSadStories & 0.40 & -0.01 & -0.19 & 0.03 \\
\hline
\end{tabular}

Note. Primary loadings of $\geq .40$ are bolded; loadings of $\geq .30$ are bolded and italicized; Anger $=$ Anger/Frustration; LowEC $=$ low effortful control; NoticeAp = Notices Appearances; Advent = Adventurous; QuietP = Quiet Play; Smiling= Smiling/Laughter; NotUpsetWithPain = Not upset by physical pain and bruises; Approach = Approach/Positive Anticipation; (Low) Activity = low Activity Level; IrritatedMistakes = Irritated by mistakes; FearDark = Fear of darkness; Soothability = Soothability/Falling Reactivity; FearofLoudNoises $=$ Fear of loud noises; NotUpsetSadStories $=$ Not upset by sad stories 


\section{Higher- and Lower-Order Factor Analyses of the Temperament in Middle Childhood Questionnaire}

Middle childhood is a crucial yet perhaps relatively underappreciated phase of human development. Indeed, it is characterized by often dramatic shifts in biological and cognitive development, as well as changes in motivation and social behavior (Campbell, 2011; Del Giudice, 2014), with profound and wide-ranging implications for the development of personality, gender identity, and emergent psychopathology (Brock \& Kochanska, 2015; Nigg, 2006). Evolutionary models of middle childhood suggest that it is a maturational stage that demands adaptive plasticity, or the ability of an organism to adjust its phenotype to match the local environment in a way that promotes survival (Del Giudice, 2014; West-Eberhard, 2003), and that children this age may therefore show heightened responsivity to environmental inputs (Del Giudice, 2014). Such sensitivity suggests that this may be a period of significant consolidation in child temperament, and that children's personality trait manifestations start to increasingly resemble those of adults through a process of increased differentiation and hierarchical integration as described by some developmental researchers (e.g., Shiner, 1998). For these reasons, the availability of valid and reliable measures of temperament during this period is crucial for gaining a better understanding of children's individual difference factors during this important developmental transition.

Based on seminal work by Thomas and Chess (1977) as well as Buss and Plomin (1984), Rothbart and colleagues (Rothbart, 1981; 2007; Rothbart, Ahadi, \& Hershey, 1994; Rothbart \& Bates, 2006) developed one of the most currently influential models of child temperament, conceptualizing it in terms of individual differences in emotional reactivity and self-regulation (i.e., the ability to modulate reactive processes). This conceptualization has been instantiated in the Temperament in Middle Childhood Questionnaire (TMCQ; Simonds, 2006; Rothbart \& Simonds; 
2004), a widely used parent-report measure of temperament spanning ages 7-10. Comprised of 157 items, 17 lower-order scales, and four higher-order factors, the TMCQ was developed via a topdown, theory-driven approach. More specifically, the TMCQ scales were adapted from temperament dimensions that had been studied in both adults and infants via the Children's Behavior Questionnaire (CBQ; Rothbart, Ahadi, Hershey, \& Fisher, 2001), the Hampton Individual Differences Questionnaire (Baker \& Victor, 2001), the Childhood Temperament and Personality Questionnaire (CTPQ; Victor, Rothbart \& Baker, 2006), and the Berkeley Puppet Interview selfreport version of the CBQ (Ablow \& Measelle, 1993). In support of its predictive validity, temperament assessed via the TMCQ has been related to important outcomes such as emerging symptoms of psychopathology, information processing biases, and emotion regulation (e.g., Herzhoff \& Tackett, 2012; Herzhoff, Tackett, \& Martel, 2013; Kotelnikova, Mackrell, Jordan, \& Hayden, 2015; Noguera, Alvarez, Carmona, \& Parra, 2015; Simonds, Kieras, Rueda, \& Rothbart, 2007). Further, the study of cultural differences in child temperament is facilitated by the fact that this measure has been translated into numerous languages (e.g., Dutch, Spanish, Italian, Portuguese, Polish, Romanian, and Taiwanese).

However, despite its popularity, the literature on the validation and psychometric properties of the TMCQ is extremely sparse. To our knowledge, such information is limited to a poster presentation by Simonds and Rothbart (2004) and an unpublished dissertation by Simonds (2006). In the poster presentation, Simonds and Rothbart (2004) reported results of a higher-order exploratory factor analysis (EFA) performed on a computerized child self-report version of the TMCQ in 30 7-year-olds, 30 8-year-olds, 44 9-year-olds, and 89 10-year-olds (total $N=193$ ); structural results were described as inconclusive by the authors. However, a four-factor solution consisting of Extraversion/Surgency, Negative Affectivity (NA), Effortful Control (EC), and 
Sociability/Affiliation obtained in this sample was subsequently described by Simonds (2006) in her unpublished dissertation. Simonds (2006) examined the psychometric properties of this earlier version of the TMCQ (both self-report and parent-report) in 147 children of unknown age, noting that TMCQ scales with $\alpha$ levels below .60 were revised, and these improved scales included in the current version of the TMCQ. This research appears to constitute the sole psychometric work on the TMCQ.

Aside from the lack of more extensive work documenting the descriptive and internal consistency statistics for the scales of the most recent version of the measure, there are other outstanding questions regarding the TMCQ. First, it is a rather lengthy measure consisting of 157 items, taking approximately 30 minutes to complete (Simonds, 2006). In noting its length, it bears mentioning that the TMCQ shows a substantial overlap with its predecessor, the CBQ (Rothbart et al., 2001), a widely used parent-report measure of temperament in preschoolers. Indeed, approximately a third of the TMCQ items were taken verbatim from the CBQ, and 13 of the 17 TMCQ scales show conceptual overlap with the CBQ. While this overlap facilitates the examination of continuity of temperament from early to middle childhood, the quality of the CBQ items appears mixed. Specifically, it was recently reported that more than half of the CBQ items did not load strongly onto a lower-order scale, and more than half of the lower-order scales did not replicate using item-level factor analysis in a very large sample of preschoolers (Kotelnikova, Olino, Klein, Kryski, \& Hayden, 2016). These results indicate that more than half of the CBQ items do not tap the constructs that they purport to tap; given that many of the CBQ items are also in the TMCQ, it is important to address their functioning in this measure.

It is also unclear how well the higher-order structure of the TMCQ maps onto the four-factor model proposed by Simonds and Rothbart (2004), given the small sample of participants used for 
the previously reported EFA (Simonds \& Rothbart, 2004) and the inconclusive results noted by the authors. The TMCQ higher-order Extraversion/Surgency factor consists of the following lowerorder scales: Activity Level, High Intensity Pleasure, Impulsivity, Shyness (reversed), and Assertiveness/Dominance; the higher-order NA factor consists of Anger/Frustration, Sadness, Fear, Discomfort, and Falling Reactivity/Soothability (reversed), and the higher-order EC factor consists of Attentional Focusing, Inhibitory Control, Low Intensity Pleasure, Perceptual Sensitivity, and Activation Control. The fourth factor, labeled Sociability/Affiliation, combines Agreeableness and Openness to Experience. In the study of the CBQ previously mentioned (Kotelnikova et al., 2016), higher-order factor analyses yielded a model that showed only minimal resemblance to that proposed by Rothbart and colleagues (2001); in particular, while NA-like and Extraversion/Surgency-like factors were found, no clear EC factor was recovered (Kotelnikova et al., 2016). Given the item overlap between the TMCQ and CBQ noted earlier, and the lack of extensive factor-analytic work on the TMCQ, further analyses of its higher-order structure are clearly needed.

With these gaps in knowledge in mind, the current study examined the lower- and higherorder structure of the TMCQ in a large sample of 9-year-olds. Our goals were two-fold: first, we aimed to identify any items that might be functioning poorly, and we also planned to compare the structures we obtained using more of a bottom-up approach to those obtained by Simonds and Rothbart (2004). Thus, we first conducted an item-level EFA to derive lower-order factors, dropping poorly functioning items (i.e., those with loadings <.40). An item-level EFA of the TMCQ provides empirically grounded information on the nature of its lower-order scales, and aids in the identification of poorly functioning items. Similarly, it allows a comparison between the 17 lower-order scales derived based on theory versus the lower-order structure developed via a 
bottom-up approach. We then conducted a higher-order EFA on these lower-order scales in order to examine the higher-order structure of the TMCQ, posited to comprise four factors in the small extant body of work on the TMCQ.

\section{Method}

\section{Participants}

Data from this study were collected as part of larger longitudinal studies conducted at two sites: London, ON, Canada (hereafter referred to as the ON sample; $N=167$ ) and Long Island, New York, USA (referred to as the NY sample; $N=487$ ). The TMCQ was designed to assess temperament traits in middle childhood, spanning ages 7 through 10. Our participants ranged in age from 8.33 to 10.92 in the NY sample (160 8-year-olds, 304 9-year-olds, and 23 10-year-olds) and from 8.89 to 10.90 in the ON sample (1 8-year-old, 143 9-year-olds, and 23 10-year-olds). Overall, although the age range of participants was determined by the availability of data (i.e., both sites had TMCQ data), the ages of children in our study represent the age range for which this measure was designed, with the exception of 7-year-olds.

The ON sample was recruited for a study of children's emotional and cognitive development through a psychology department database of research volunteers, and advertisements placed in local newspapers and online. The NY sample was recruited through commercial mailing lists for a study of child temperament. In both samples, children with major psychological and medical concerns, as determined by trained study personnel during recruitment, were ineligible. Children in both samples performed within the normal range on the Peabody Picture Vocabulary Test, Fourth Edition (PPVT-IV; Dunn \& Dunn, 2007). Overall, participant demographic characteristics were similar across the two samples, suggesting that combining the two datasets for analyses was reasonable (Table 1). We also compared mean TMCQ scale scores between the two 
samples (see next section; Table 2). To further verify the appropriateness of combining the two samples, we conducted specific tests of structural invariance, as described later in the paper.

Finally, we also conducted tests of structural invariance across gender.

\section{Assessment of Temperament}

Primary caregivers completed the TMCQ as a measure of their child's temperament at ages 9 at both sites. The current form of the TMCQ ( $3^{\text {rd }}$ version) consists of 157 items rated on a 5-point Likert scale ranging from 1 (almost always untrue) to 5 (almost always true). Scale means and internal consistency statistics are presented in Table 2, and are comparable to those reported in the extant literature (Simonds, 2006; Simonds et al., 2007; Simonds \& Rothbart, 2004). Scale distributions were generally good (see Table 2).

\section{Between-sample differences.}

Independent-sample t-tests were conducted to examine mean-level differences in scale scores between the two sites, with six TMCQ scales differing significantly (Table 2). Primary caregivers in the NY sample tended to rate their children higher on all of the scales with significant differences, except for Sadness. However, effect sizes for between-sample mean differences on the TMCQ scales were quite small (Table 2), and mean differences on scale scores do not influence structural analyses (Goodwin \& Leech, 2006). Also, similar Ms and SDs to those in our samples have been reported by the TMCQ developers in other samples (Simonds, 2006; Simonds et al., 2007).

\section{Proposed Analyses}

As a first step, items were subjected to EFAs using Mplus 7 statistical software (Muthen \& Muthen, 1998-2012); to our knowledge, this important step in scale development has never before been conducted with the TMCQ, and permits the examination of the extent to which the original 
scales, constructed based on expert consensus, map onto an empirical approach to scale development. Further, the alternative, a confirmatory factor analytic approach is too restrictive for a complex measure like the TMCQ. More specifically, the CFA approach of fixing many or all crossloadings of observed indicators to zero may force a researcher to specify a model that is more parsimonious than appropriate for the data (Asparouhov \& Muthen, 2009; Hopwood \& Donnellan, 2010). Such models often do not fit the data well, requiring extensive model modifications to improve fit. These extensive post-hoc model modifications result in a CFA that is more exploratory than confirmatory in nature (Asparouhov \& Muthen, 2009). For our EFA parameter estimation procedures, we used the maximum likelihood robust (MLR) estimator (Muthen \& Muthen, 19982012) and the geomin oblique rotation method recommended by Browne (2001). This rotation was used for both higher- and lower-order factor analyses. The Kaiser-Guttman criterion for factor retention in an EFA indicates that factors with eigenvalues over 1 should be retained. We also performed a parallel analysis (O’Connor, 2000) in which we ran a simulation with 1000 replications to determine what the eigenvalues would be if there were the same number of cases and variables, but the data were random. If the eigenvalue for a factor from our real data was lower than expected due to chance (i.e., those produced from the parallel analysis), then that factor would not be interpreted as capturing any latent traits present in the data.

The obtained lower-order factors were then computed as averages of their corresponding items with loadings of $\geq .40^{7}$. Next, to examine the higher-order structure of the TMCQ, the obtained lower-order factors were subjected to a series of EFAs extracting three to five factors. The decision to focus on three- to five- factor models was based on the extant literature on personality

\footnotetext{
${ }^{7}$ Although a cut-off of .30 is sometimes used to designate an acceptable loading in EFAs, use of a more stringent cutoff of .40 is also common (Briggs \& MacCallum, 2003; Comrey, 1973; Hogarty, Kromrey, Ferron, \& Hines, 2004). Notably, similar structures were recovered in our sample using less stringent loading cut-offs, i.e., .35 and .32 .
} 
and temperament structure (Caspi \& Shiner, 2006; Costa \& McCrae, 2008; De Pauw \& Mervielde, 2010; Rothbart et al., 2001; Watson \& Clark, 1993), which suggests that most of the variance in both child and adult temperament/personality is accounted for by three-to-five broad factors (Markon, Kruger, \& Watson, 2005), as well as the purported four-factor structure of the TMCQ (Simonds \& Rothbart, 2004). We relied on indices of model fit conventionally available in confirmatory factor analysis to compare higher-order EFA models to one another. We used comparative fit index (CFI) values of above .90 and .95 as indices of acceptable and excellent fit (Hu \& Bentler, 1999). Additionally, we treated root-mean-square of approximation (RMSEA) values that were lower than .05 as indicating a close fit, with values up to 0.08 indicating acceptable fit (Marsh, Hau, \& Wen, 2004). Models with varying numbers of factors were compared using the Satorra-Bentler chi-square difference test (Asparouhov \& Muthen, 2009). Due to our large sample size, we adopted a more stringent test of $p<.01$ for comparisons between models for deciding between different models.

As a final step, we followed a step-wise procedure outlined by Little (2013) to ascertain structural invariance of the higher order solution across the two samples. We tested for weak, strong, and strict invariance across the two samples (ON and NY). Tests of weak factorial invariance involve setting each corresponding loading in the two samples to be equal; however, variances, intercepts, and residuals are allowed to vary. Testing strong invariance involves imposing equality constraints on each observed intercept across samples, and tests of strict invariance impose equality constraints on residuals across samples (Little, 2013). Higher levels of factorial invariance are acceptable if the change in model fit from a lower to higher level of invariance is negligible, i.e., if the change in RMSEA and CFI does not exceed .015 (Chen, 2007). 


\section{Results}

\section{Item-Level Exploratory Factor Analysis}

Results of an item-level $\mathrm{EFA}^{8}$ in the combined sample are shown in Table 3. Initially, this analysis identified 37 factors with eigenvalues over 1; however, only 14 factors with larger eigenvalues than the simulated data sets were extracted based on the results of the parallel analysis (O’Connor, 2000). Model fit of the 14-factor EFA solution was deemed good based on the RMSEA (.03); however, the CFI (.84) was weak. Of the 157 items analyzed, 59 items had primary loadings $<.40$, and were excluded from subsequent analyses. Items that were excluded from further analyses came from the following original TMCQ scales: Activation Control (nine items), High Intensity Pleasure (eight items), Discomfort (seven items), Fantasy/Openness (five items), Low Intensity Pleasure (five items), Soothability/Falling Reactivity (five items), Fear (four items), Affiliation (four items), Inhibitory Control (four items), Assertiveness/Dominance (three items), Perceptual Sensitivity (three items), Impulsivity (one item), and Sadness (one item). Given that most of the original scales consist of ten or fewer items, excluding more than half of the items from these scales suggests that these constructs may not be adequately represented (e.g., Discomfort and High Intensity Pleasure). Six additional items were excluded as they had high secondary loadings (i.e., $\geq .30$ ), and may therefore not differentiate clearly between the lower-order factors on which they load.

After these steps, 92 items remained with primary loadings $\geq .40$ and no secondary loadings z.30. Of the 14 factors extracted, one factor was excluded from further analyses as it consisted of a single item ("Gets angry when s/he makes a mistake"). Thus, 13 factors remained for subsequent

\footnotetext{
${ }^{8} \mathrm{We}$ also conducted a CFA the original 17 TMCQ scales as well as the original higher-order four-factor structure (Surgency, Negative Affectivity, Effortful Control, and Sociability/Affiliation; Simonds, 2006). Our results were not supportive of these lower- and higher-order structures. In particular, a CFA model of the original 17 TMCQ scales did not converge, and a CFA model of the original four higher-order factors had a very poor fit (RMSEA $=.18 ; \mathrm{CFI}=.58$ ).
} 
higher-order EFA analyses (Table 3). Nine of these lower-order factors resembled the original TMCQ scales in their content (i.e., Impulsivity, Activity Level, Attentional Focusing, Shyness, Sadness, Perceptual Sensitivity, Assertiveness/Dominance, Affiliation, and Fantasy/Openness). However, Assertiveness/Dominance, Sadness, Affiliation, and Fantasy/Openness consisted of only about half of the original items. Bivariate associations between the newly derived 13 factors calculated based on the average of their respective items and the original 17 TMCQ scales are presented in Table 4. Correlations between the nine lower-order factors that were similar to the TMCQ scales and these original scales ranged from .77 to .97 . The rest of the 13 lower order factors consisted of combinations of items from different original TMCQ scales (e.g., Anger and Sadness) or were too narrow to be considered temperament traits (e.g., Fear of Needles and Fear of Darkness/Burglars).

\section{Higher-Order Exploratory Factor Analysis}

The 13 factors identified using the item-level EFA were subjected to a higher-order EFA with a geomin rotation, using MLR estimator; as previously noted, based on theory (Caspi \& Shiner, 2006; De Pauw \& Mervielde, 2010; Costa \& McCrae, 2008; Rothbart et al., 2001; Simonds \& Rothbart, 2004; Watson \& Clark, 1993), three to five factors were extracted. A three-factor model yielded the only acceptable solution (four- and five-factor models did not converge) and had an acceptable fit $(\mathrm{RMSEA}=.06 ; \mathrm{CFI}=.93$; see Table 5$)$. The first factor of this model combined lower-order factors tapping Impulsivity and Anger/Sadness, the second factor of this model was consistent with Negative Affectivity, and the third factor from this model combined lower-order factors of Affiliation, Fantasy/Openness, and Assertiveness/Dominance, representing a combination of Extraversion/Surgency, Agreeableness, and Openness to Experience traits from the five-factor model of adult personality (McCrae \& Costa, 1997). 
We also tested for invariance of temperament structures across the two samples (i.e., ON and NY) to determine whether the three-factor solution derived in the joint sample was acceptable ${ }^{9}$. We started by fitting a three-factor model that was equivalent in its configuration in both samples; this baseline model had an acceptable fit $(\mathrm{RMSEA}=.07 ; \mathrm{CFI}=.93)$. Table 6 outlines the results of structural invariance tests (i.e., weak, strong, and strict) that were applied sequentially to the threefactor baseline model. Higher levels of factorial invariance are acceptable if the change in model fit from a lower to higher level of invariance is negligible, i.e., if the change in RMSEA and CFI does not exceed .015 (Chen, 2007). Setting each corresponding loading in the two samples to be equal, while allowing variances, intercepts, and residuals to vary (test of weak invariance) did not result in a significant change in the fit indices $(\triangle \mathrm{RMSEA}=.009$ and $\triangle \mathrm{CFI}=.002)$. Imposing equality constraints on each observed intercept across samples (test of strong invariance) also did not yield a significant change in both of the fit indices $(\triangle \mathrm{RMSEA}=.010$ and $\triangle \mathrm{CFI}=.037)$. Finally, imposing equality constraints on residuals across samples (test of strict invariance) also did not result in a significant change in the fit indices $(\triangle \mathrm{RMSEA}=.001$ and $\triangle \mathrm{CFI}=.009)$. Overall, these results indicated that imposition of weak, strong, and strict invariance of the solutions did not significantly diminish model fit. Thus, the factorial structure of the instrument is equivalent across the two samples.

\section{Discussion}

We used a bottom-up approach to examining higher- and lower-order structures of a widely

\footnotetext{
${ }^{9}$ Aside from testing the newly derived three-factor model for invariance of temperament structures across the two samples, we also tested this model for invariance across child sex. The three-factor model passed the weak (loadings) invariance test. This model also passed the strong (intercepts) partial invariance test. Based on the modification indices, intercept equality constraints had to be relaxed for the three lower-order factors: Fantasy/Openness, Affiliation, and Low Attentional Focusing. Following these modifications, the model also passed the strict (residuals) invariance test. These analyses are available upon request from the first author.
} 
used measure of temperament in middle childhood, the TMCQ (Simonds, 2006; Simonds \& Rothbart, 2004). To our knowledge, our item-level and higher-order factor analyses of empirically derived lower-order factors of this measure are unique in the literature; this is likely due to the difficulty in acquiring a sufficient sample size for item-level analyses of a measure as lengthy as the TMCQ. Our findings indicated that a large number of TMCQ items (65 items or $42 \%$ of the items) did not clearly load onto a lower-order scale. Critically, several lower- and higher-order temperament dimensions (e.g., fear, anger, sadness) that are prominent in most major temperament models (Caspi \& Shiner, 2006; De Pauw, Mervielde, \& Van Leeuwen, 2009; Rothbart et al., 2001) were poorly represented in the structures derived in our sample, as items putatively tapping these constructs failed to load onto scales. Also, the higher-order structure of temperament in middle childhood did not bear resemblance to the four-factor structure posited by Simonds and Rothbart (2004), nor did it resemble other prominent models of child temperament and personality (e.g., Capsi \& Shiner, 2006; De Pauw et al., 2009; Rothbart et al., 2001).

While the TMCQ is a lengthy measure, our findings indicate that a large number of items (65 items out of original 157) did not contribute to lower-order scales, suggesting that the TMCQ is longer than necessary and that many of its items are not effective indicators of the constructs they purport to tap. These item-level results are not surprising. A previous study from our group (Kotelnikova et al., 2016) showed that less than half of the original 195 CBQ items loaded onto lower-order scales. The TMCQ was developed via a top-down approach as an adaptation of the CBQ for older children, and approximately a third of the TMCQ items were taken verbatim from the CBQ. Thirty-one percent of these common items also failed to load onto TMCQ lower-order factors.

Item-level EFAs indicated that approximately half of the factors resembled the original 
TMCQ scales (i.e., contained similar items) created by Simonds and Rothbart (2004); these were TMCQ Impulsivity, Activity Level, Attentional Focusing, Shyness, Affiliation, Perceptual Sensitivity, Fantasy/Openness, Sadness, and Assertiveness/Dominance. Thus, item-level analyses yielded only nine scales resembling those generated by the developers of the TMCQ based on expert opinion. The remaining lower-order scales derived from EFA were comprised of items from multiple original TMCQ scales (e.g., the Anger/Sadness lower-order factor we found was a mix of items from the original Anger and Sadness scales), or did not represent constructs broad enough to be deemed temperament traits (e.g., fear of dark and burglars, fear of needles, liking stories).

Putatively distinct facets of NA were poorly differentiated in our analyses. In particular, we recovered a lower-order factor that was comprised of items from both the original Anger and Sadness scales. Difficulty in deriving clear lower-order Anger and Sadness factors may be related to the overlapping language used in the items that tap these constructs in the current version of the TMCQ. Specifically, most of these items describe children's affective responses (either anger or sadness) to similar events (e.g., item 94 "gets angry when s/he has trouble with a task" and item 107 "seems to feel down when unable to accomplish a task"). Of the 15 items comprising the empirically derived Anger/Sadness lower-order factor in our analyses, only six items refer specifically to anger (items 53,61,87, 94, 110, and 146), and of these six items, only two (items 110 and 146) do not share similar language with sadness items. Should revisions of the TMCQ be pursued, it may be useful to create additional anger-specific items that do not overlap in language with items tapping sadness. However, it is also possible that children's anger and sadness are highly co-occurring, which would make it difficult for parents to make fine-grained distinctions between the two emotions. Consistent with this possibility, recent behavior genetic studies (e.g., Clifford, Lemery-Chalfant, \& Goldsmith, 2015) have shown that anger and sadness share greater variance in 
terms of genetic and shared environmental influences than anger does with other facets of negative affect. Finally, studies of observed temperament in preschoolers (Dyson, Olino, Durbin, Goldsmith, \& Klein, 2012; Kotelnikova, Kryski, \& Hayden, 2015) have also provided evidence that anger and sadness cluster together. If so, striving to create scales that tap these as distinct constructs may prove challenging.

Another key aspect of NA, child fear, may also be poorly tapped by the TMCQ. Specifically, the two fear-like scales that we recovered were too narrow in their scope to be considered temperament traits; specifically, the two scales are Fear of Dark and Burglars and Fear of Needles/Shyness. Indeed, half of items belonging to the original TMCQ scale tapping fear were excluded due to low loadings, suggesting that a revision of the TMCQ should include additional fear items that better tap this important aspect of child temperament.

Analyses of lower-order factors also showed that TMCQ EC items failed to comprise the various EC facets posited by Simonds and Rothbart (2004; Attentional Focusing, Inhibitory Control, Activation Control, Low Intensity Pleasure, and Perceptual Sensitivity). We were able to recover only two scales resembling these, Low Attentional Focusing and Perceptual Sensitivity. Further, many items tapping EC facets were excluded due to low loadings; specifically, ten of the original 15 Activation Control items, four of the eight Inhibitory Control items, six of the eight Low Intensity Pleasure items, and three of the ten Perceptual Sensitivity items were excluded due to low loadings. The remaining Inhibitory Control items loaded on the Impulsivity factor, while Activation Control items loaded primarily with Attentional Focusing items, possibly because these Activation Control items (20,89, and 93) refer specifically to homework completion. The remaining Low Intensity Pleasure items comprised a three-item factor too narrow to be considered a temperament trait (i.e., Likes Stories). Revision of the TMCQ EC items may be needed in order to tap the various 
lower-order facets proposed by Simonds and Rothbart (2004). Another possibility is that the lowerorder EC dimensions of Simonds and Rothbart's model do not reflect the multifaceted nature of EC. Given that extant literature supports the notion that EC is a multidimensional construct, there may be alternative ways of parsing EC that more accurately represent the facets of this higher-order construct (e.g., Murray \& Kochanska, 2002).

At the higher-order level, we recovered a three-factor structure consisting of Impulsivity/NA, NA, and the third factor combining facets of Fantasy/Openness, Assertiveness/Dominance, and Affiliation. This structure did not bear close resemblance to the original four-factor model proposed by Simonds and Rothbart (2004), comprised of Extraversion/ Surgency, NA, EC, and Sociability/Affiliation. Conceptually, major models of personality and temperament view NA and EC as distinct concepts (e.g., Buss \& Plomin, 1984; Caspi \& Shiner, 2006; De Pauw et al., 2009; McCrae \& Costa, 1997; Rothbart et al., 2001; Tellegen, 1985; Watson \& Clark, 1993). However, in our additional exploratory structural equation modeling analyses not reported in full here, constraining loadings of NA-related lower-order factors on higher-order Factor 2 and preventing them from loading on Factor 1 to generate a cleaner structure resulted in an unacceptably poor fit. The third factor in our model was also a mixture of constructs representing lower-order scales tapping Extraversion/Surgency as well as "likes stories" (an unusual lower-order scale), Fantasy/Openness, and Perceptual Sensitivity. Other information published on the structure of the TMCQ was drawn from computerized child self-report (Simonds \& Rothbart, 2004) rather than parent report, which could contribute to structural differences. Having said that, given that many TMCQ items were dropped due to low scale loadings and that our lower-order scales were substantially different from those in the original model, it is not surprising that the higher-order structure would differ. 
The TMCQ may need new items, as numerous theoretically important, distinct constructs did not emerge as separable lower-order factors, including all of the NA facets, such as Anger/Frustration, Sadness, Fear, Discomfort, and Soothability/Falling Reactivity and some of the EC facets, such as Activation Control, Inhibitory Control, and Low Intensity Pleasure. Indeed, itemlevel EFA analyses showed that more than half of the items from each of these scales did not differentiate between the concepts they purported to tap. Supplementing the better-functioning existing TMCQ items with newer items that tap underrepresented constructs could prove useful in revising the TMCQ. An extended item pool could then be validated in large samples of children at the item- and higher-order levels using exploratory factor analysis and measurement invariance testing to ascertain validity of the scales and high-order factors and ensure comparable item functioning for boys and girls. Such structural analyses could be followed up by IRT for a more detailed examination of item functioning and further refining of the measure.

Our study is the first item-level analysis of a widely used parent-report measure of temperament in middle childhood. Compared to the analytic methods used in the original scale development (Simonds \& Rothbart, 2004), the approach we used is less subject to influence by item properties (Goodwin \& Leech, 2006). The large sample size is also a significant strength. However, our study had several limitations. First, the CFI values in our item-level EFA analysis did not reach the recommended value of .90 (Bentler, 1990). However, other fit statistic (i.e., RMSEA) indicated good model fit. Second, despite the acceptable fit coefficients of the higher-order model presented in Table 4, there were relatively few lower-order factors with high loadings. The main implication of the absence of high loading lower-order factors is that the interpretability of the broader factors is somewhat limited; we therefore tried to be agnostic in how we describe these factors throughout the manuscript. Overall, it cannot be said that the higher-order structures capture most of the scales. 
Also, some of our EFAs included factors with only two items; such factors may not be especially stable or replicate in future analyses. Finally, both samples were racially/ethnically homogenous and largely middle- and upper-class, which may limit the generalizability of our findings to ethnically diverse children.

Rothbart's family of temperament measures have been extensively used in studies of child temperament for decades. Rothbart's and colleagues' paper on validating the Children's Behavior Questionnaire has been cited over 900 times (Rothbart et al., 2001). In order to facilitate longitudinal research on child temperament, it is extremely important to validate measures that represent an extension of the CBQ to older age groups (i.e., the TMCQ). These measures have also been translated into numerous other languages, facilitating research on child temperament in other countries (e.g., the TMCQ has been translated into eight different languages). The TMCQ is presently the least validated of all Rothbart's measures of child temperament and, therefore, it desperately requires more research on its psychometric properties and structure. Our study provides important new information on a widely used measure of temperament in middle childhood. The results of our study suggest that revisions of the TMCQ are needed, which could include eliminating poorly functioning items and developing new items to tap important temperamental constructs that may not be currently represented well (e.g., NA facets), as well as reconsidering the number of higher-order factors required to fully represent the domain of temperament in middle childhood. These revisions may be of great benefit to researchers in the fields of child development, developmental psychopathology, and child temperament. 


\section{References}

Ablow, J. C., \& Measelle, J. R. (1993). The Berkeley Puppet Interview: Administration and Scoring Manuals. Berkeley: University of California.

Asparouhov, T., \& Muthén, B. (2009). Exploratory structural equation modeling. Structural Equation Modeling, 16, 397-438. doi:10.1080/10705510903008204

Baker, S. R., \& Victor, J. B. (2001, August). African American children's personality: A confirmatory factor analysis. Presentation at the Xth European Conference of Development Psychology. Uppsala, Sweden.

Bentler, P. M. (1990). Comparative fit indexes in structural models. Psychological Bulletin, 107, 238-246.

Briggs, N. E., \& MacCallum, R. C. (2003). Recovery of weak common factors by maximum likelihood and ordinary least squares estimation. Multivariate Behavioral Research, 38, 2556. doi:10.1207/S15327906MBR3801_2

Brock, R. L., \& Kochanska, G. (2015). Decline in the quality of family relationships predicts escalation in children's internalizing symptoms from middle to late childhood. Journal of Abnormal Child Psychology, doi:10.1007/s10802-015-0008-9

Browne, M. W. (2001). An overview of analytic rotation in exploratory factor analysis. Multivariate Behavioral Research, 36, 111-150.

Buss, A. H. \& Plomin, R. (1984). Temperament: Early developing personality traits. Hillside, NJ: Erlbaum.

Campbell, B. C. (2011). An introduction to the special issue on middle childhood. Human Nature, 22, 247-248. Doi:10.1007/s12110-011-9118-4

Caspi, A., \& Shiner, R. L. (2006). Personality development. Hoboken, NJ, US: John Wiley \& 
Sons Inc, Hoboken, NJ.

Chen, F. F. (2007). Sensitivity of goodness of fit indexes to lack of measurement invariance. Structural Equation Modeling, 14, 464-504.

Clifford, S., Lemery-Chalfant, K., \& Goldsmith, H. H. (2015). The unique and shared genetic and environmental contributions to fear, anger, and sadness in childhood. Child Development, doi:10.1111/cdev.12394

Cohen, J. (1988). Statistical power analysis for the behavioral sciences (2 ${ }^{\text {nd }}$ ed.). Hillsdale, NJ: Lawrence Earlbaum Associates.

Comrey, A.L. (1973). A first course in factor analysis. New York: Academic Press.

Costa, P., \& McCrae, R. (2008). The revised NEO personality inventory (NEO-PI-R). In G. Boyle, G. Matthews, \& D. Saklofske (Eds.), The SAGE handbook of personality theory and assessment: Volume 2 - Personality measurement and testing. (pp. 179-199). London: SAGE Publications Ltd. doi: 10.4135/9781849200479.n9

Del Giudice, M. (2014). Middle childhood: An evolutionary-developmental synthesis. Child Development Perspectives, 8, 193-200. doi:10.1111/cdep.12084

De Pauw, S. S. W., \& Mervielde, I. (2010). Temperament, personality and developmental psychopathology: A review based on the conceptual dimensions underlying childhood traits. Child Psychiatry and Human Development, 41, 313-329. doi:10.1007/s 10578-009$0171-8$

De Pauw, S. S. W., Mervielde, I., \& Van Leeuwen, K. G. (2009). How are traits related to problem behavior in preschoolers? similarities and contrasts between temperament and personality. Journal of Abnormal Child Psychology,37, 309-325. doi: 10.1007/s10802-0089290-0 
Dunn, L. M., \& Dunn, L. M. (2007). Peabody Picture Vocabulary Test (4 $^{\text {th }}$ ed.). Circle Pines, MN: American Guidance Service.

Dyson, M.W., Olino, T.M., Durbin, C.E., Goldsmith, H.H., \& Klein, D. N. (2012). The structure of temperament in preschoolers: A two-stage factor analytic approach. Emotion, 12, 44-57.

Goodwin, L. D., \& Leech, N. L. (2006). Understanding correlation: Factors that affect the size of r. Journal of Experimental Education, 74, 251-266. doi:10.3200/JEXE.74.3.249-266

Herzhoff, K., \& Tackett, J. L. (2012). Establishing construct validity for openness-to-experience in middle childhood: Contributions from personality and temperament. Journal of Research in Personality, 46, 286-294. doi:10.1016/j.jrp.2012.02.007

Herzhoff, K., Tackett, J. L., \& Martel, M. M. (2013). A dispositional trait framework elucidates differences between interview and questionnaire measurement of childhood attention problems. Psychological Assessment, 25, 1079-1090. doi:10.1037/a0033008

Hogarty, K. Y., Kromrey, J. D., Ferron, J. M., \& Hines, C. V. (2004). Selection of variables in exploratory factor analysis: An empirical comparison of a stepwise and traditional approach. Psychometrika, 69, 593-611. doi:10.1007/BF02289857

Hopwood, C. J., \& Donnellan, M. B. (2010). How should the internal structure of personality inventories be evaluated? Personality and Social Psychology Review, 14, 332-346. doi:10.1177/1088868310361240

Hu, L., \& Bentler, P. M. (1999). Cutoff criteria for fit indexes in covariance structure analysis: Conventional criteria versus new alternatives. Structural Equation Modeling, 6, 155. doi:10.1080/10705519909540118

Kotelnikova, Y., Kryski, K.R., \& Hayden, E.P. (2015). Structure of Observed Temperament in 
Preschoolers and Associations with Internalizing and Externalizing Symptoms. Poster presented at the Society for Research in Psychopathology Annual Meeting, New Orleans, Louisiana, USA.

Kotelnikova, Y., Mackrell, S. V. M., Jordan, P. L., \& Hayden, E. P. (2015). Longitudinal associations between reactive and regulatory temperament traits and depressive symptoms in middle childhood. Journal of Clinical Child and Adolescent Psychology, 44, 775-786. doi:10.1080/15374416.2014.893517

Kotelnikova, Y., Olino, T.M., Klein, D.N., Kryski, K.R., \& Hayden, E.P. (2016). Higherand lower-order factor analyses of the Children's Behavior Questionnaire in early and middle childhood. Psychological Assessment, 28. 92-108. doi: 10.1037/pas0000153

Little, T. D. (2013). Longitudinal structural equation modeling Guilford Press, New York, NY.

Markon, K. E., Krueger, R. F., \& Watson, D. (2005). Delineating the structure of normal and abnormal personality: An integrative hierarchical approach. Journal of Personality and Social Psychology, 88, 139-157. doi:10.1037/0022-3514.88.1.139

Marsh, H. W., Hau, K. T., \& Wen, Z. (2004). In search of golden rules: Comment on hypothesis testing approaches to setting cutoff values for fit indexes and dangers in over-generalizing Hu \& Bentler's (1999) findings. Structural Equation Modeling, 11, 320-341.

McCrae, R. R., \& Costa, P. T. (1997). Personality trait structure as a human universal. American Psychologist, 52, 509-516. doi:10.1037/0003-066X.52.5.509

Murray, K. T., \& Kochanska, G. (2002). Effortful control: Factor structure and relation to externalizing and internalizing behaviors. Journal of Abnormal Child Psychology, 30, 503514. doi:10.1023/A:1019821031523

Muthén, L.K. and Muthén, B.O. (1998-2012). Mplus User's Guide. Seventh Edition. 
Los Angeles, CA: Muthén \& Muthén.

Nigg, J. T. (2006). Temperament and developmental psychopathology. Journal of Child Psychology and Psychiatry, 47, 395-422. doi:10.1111/j.1469-7610.2006.01612.x

Noguera, C., Álvarez, D., Carmona, E., \& Parra, L. (2015). Temperament and negative semantic priming in children 7 to 12 years old. Child Neuropsychology, 21, 302-313. doi:10.1080/09297049.2014.913558

O'Connor, B. P. (2000). SPSS and SAS programs for determining the number of components using parallel analysis and Velicer's MAP test. Behavior Research Methods, Instrumentation, and Computers, 32, 396-402.

Rothbart, M. K. (1981). Measurement of temperament in infancy. Child Development, 52(2), 569-578.

Rothbart, M. K. (2007). Temperament, development, and personality. Current Directions in Psychological Science, 16, 207-212. doi:10.1111/j.1467-8721.2007.00505.x

Rothbart, M. K., Ahadi, S. A., \& Hershey, K. L. (1994). Temperament and social behavior in childhood. Merrill-Palmer Quarterly, 40, 21-39.

Rothbart, M. K., Ahadi, S. A., Hershey, K. L., \& Fisher, P. (2001). Investigations of temperament at three to seven years: The children's behavior questionnaire. Child Development, 72, 1394-1408. doi:10.1111/1467-8624.00355

Rothbart, M.K. \& Bates, J.E. (2006). In Handbook of child psychology: Vol. 3 Social, emotional, and personality development (6th ed.), by Rothbart, Mary K., Bates, John E., 99166. Hoboken, NJ, US: John Wiley \& Sons Inc.

Shiner, R. L. (1998). How shall we speak of children's personality traits in middle childhood? A preliminary taxonomy. Psychological Bulletin, 124, 308-332. 
Simonds, J. (2006). The role of reward sensitivity and response: Execution in childhood extraversion. Unpublished doctoral dissertation, University of Oregon.

Simonds, J. \& Rothbart, M. K. (2004). The Temperament in Middle Childhood Questionnaire (TMCQ): A computerized self-report measure of temperament for ages 7- 10. Poster session presented at the Occasional Temperament Conference, Athens, GA.

Simonds, J., Kieras, J., Rueda, M., \& Rothbart, M. (2007). Effortful control, executive attention, and emotional regulation in 7-10-year-old children. Cognitive Development, 22, 474-488. doi:10.1016/j.cogdev.2007.08.009

Tellegen, A. (1985). Structure of mood and personality and their relevance to assessing anxiety, with an emphasis on self-report. In A. H. Tuma \& J. D. Maser (Eds.), Anxiety and the anxiety disorders (pp. 681-706). Hillsdale, NJ: Erlbaum.

Thomas, A., \& Chess, S. (1977). Temperament and development. Brunner/Mazel, Oxford.

Victor, J. B., Rothbart, M. K., \& Baker, S. R. (2006). Identifying temperamental components of developing personality through combining temperament and personality scales. Unpublished Manuscritpt.

Watson, D., \& Clark, L. A. (1993). In Wegner D. M., Pennebaker J. W. (Eds.), Behavioral disinhibition versus constraint: A dispositional perspective. Englewood Cliffs, NJ, US: Prentice-Hall, Inc, Englewood Cliffs, NJ.

West-Eberhard, M. J. (2003). Developmental plasticity and evolution. New York, NY: Oxford University Press. 
Table 1.

Sample Descriptive Statistics

\begin{tabular}{l|c|c}
\hline Sample: & ON & NY \\
\hline$N$ & 167 & 487 \\
$M$ child age (SD) & $9.62(.38)$ & $9.18(.40)$ \\
$\%$ boys & $43 \%$ & $54 \%$ \\
$M$ PPVT (SD) & $112(12)$ & $108(11)$ \\
$\%$ of caregivers who were mothers & $87 \%$ & $93 \%$ \\
$M$ caregiver age $(S D)$ & $33.87(12.88)$ & $41.22(5.25)$ \\
Ethnicity: & & \\
Caucasian & $89 \%$ & $80 \%$ \\
African & -- & $5 \%$ \\
Asian & $3 \%$ & $3 \%$ \\
Hispanic/Latino & -- & $12 \%$ \\
Other & $7 \%$ & -- \\
Missing data & $1 \%$ & -- \\
Family income: & & \\
$\quad<20,000$ & $6 \%$ & $2 \%$ \\
$20,001-40,000$ & $7 \%$ & $5 \%$ \\
$40,001-70,000$ & $26 \%$ & $22 \%$ \\
$70,001-100,000$ & $26 \%$ & $16 \%$ \\
$>100,000$ & $27 \%$ & $55 \%$ \\
Missing data & $8 \%$ & \\
\hline
\end{tabular}

Note. ON - sample collected in London, ON; NY - sample collected in Long Island, New York, USA. 
Table 2.

Descriptive Statistics for the 17 Original TMCQ Scales

\begin{tabular}{lllllllllllll}
\hline \multicolumn{1}{c}{ TMCQ Scale: } & \multicolumn{1}{c}{ ON } & \multicolumn{1}{c}{ NY } & \multicolumn{1}{c}{ NY } \\
\hline & & $M$ & $S D$ & $\alpha$ & Skew. & Kurt. & $M$ & SD & $\alpha$ & Skew. & Kurt. & \\
\hline 1. ActivCn & $3.42^{* *}$ & .49 & .80 & -.49 & 1.00 & $3.57^{* *}$ & .49 & .77 & .03 & -.10 & .27 \\
2. Activity & 3.86 & .67 & .89 & -.05 & -1.10 & 3.95 & .68 & .89 & -.60 & -.08 & .13 \\
3. Affiliation & 4.20 & .45 & .77 & -.59 & .08 & 4.19 & .49 & .78 & -.57 & -.09 & .01 \\
4. Anger & 2.79 & .63 & .79 & .26 & .84 & 2.76 & .76 & .85 & .15 & -.33 & .04 \\
5. Assertiv. & 3.53 & .48 & .74 & .45 & .29 & 3.59 & .52 & .72 & .13 & -.06 & .12 \\
6. AttnFocus & $3.41^{*}$ & .83 & .92 & -.35 & .05 & $3.58^{*}$ & .91 & .92 & -.56 & -.20 & .17 \\
7. Discomfort & 2.30 & .60 & .76 & .44 & -.30 & 2.38 & .61 & .71 & .31 & .02 & .01 \\
8. Fantasy & 4.07 & .53 & .80 & -.61 & -.10 & 4.08 & .53 & .75 & -.53 & .05 & .01 \\
9. Fear & $2.32^{*}$ & .67 & .75 & .51 & -.26 & $2.47^{*}$ & .67 & .75 & .43 & .08 & .19 \\
10. HighPL & 3.41 & .60 & .82 & -.06 & -.50 & 3.40 & .58 & .77 & -.09 & -.40 & .02 \\
11. Impulsivity & 2.67 & .67 & .90 & .18 & -.01 & 2.61 & .71 & .90 & .55 & .29 & .07 \\
12. InhibCn & 3.44 & .56 & .73 & -.30 & -.12 & 3.45 & .61 & .73 & -.32 & .08 & .01 \\
13. LowPL & 3.66 & .50 & .67 & -.34 & .15 & 3.64 & .57 & .68 & -.25 & -.09 & .04 \\
14. PerSen & 3.31 & .61 & .82 & -.57 & .58 & 3.36 & .58 & .78 & -.08 & .06 & .08 \\
15. Sadness & $2.50^{* *}$ & .53 & .77 & .41 & -.15 & $2.32^{* *}$ & .55 & .81 & .58 & .67 & .30 \\
16. Shyness & $2.57^{* *}$ & .77 & .83 & .14 & -.60 & $2.29^{* *}$ & .83 & .84 & .56 & .03 & .30 \\
17. Sooth. & $3.66^{* *}$ & .59 & .76 & -.45 & -.32 & $3.89^{* *}$ & .65 & .83 & -.65 & .39 & .32 \\
\hline
\end{tabular}

Note. The table depicts between sample comparisons of the TMCQ scale means; ${ }^{* *} p<.01 ; * p<.05$; $d=.30$ is a small, and $d=.50$ is a medium effect (Cohen, 1988); ActivCn = Activation Control; Activity = Activity Level; Anger = Anger/Frustration; Assertiv. = Assertiveness/Dominance; AttnFocus = Attentional Focusing; Fantasy = Fantasy/Openness; HighPL = High Intensity Pleasure; InhibCn = Inhibitory Control; LowPL = Low Intensity Pleasure; PerSen = Perceptual Sensitivity; Sooth $=$ Soothability/Falling Reactivity 
Table 3.

Exploratory Factor Analysis of the TMCQ Items

\begin{tabular}{|c|c|c|c|c|c|c|}
\hline Item\# & Scale & Item Description & Impulsivity & Activity & LowAttnFocus & Ang/Sad \\
\hline 16 & Impulsivity & SaysFirstThing & 0.76 & & & \\
\hline 25 & Impulsivity & SaysFirstThingtoMind & $\underline{0.75}$ & & & \\
\hline 130 & Impulsivity & RushesIntoActivity & $\overline{0.74}$ & & & \\
\hline 14 & Impulsivity & TouchWithoutPermission & $\overline{0.61}$ & & & \\
\hline 83 & AttnFocus & RushesNewThings & $\overline{0.52}$ & & & \\
\hline 108 & Impulsivity & TroubleBecauseNoThinking & $\underline{0.51}$ & & & \\
\hline 22 & Impulsivity & Interrupts & 0.49 & & & \\
\hline 124 & Impulsivity & Grabs & $\overline{0.46}$ & & & \\
\hline 143 & InhibCn & HardSlowingDownToWalk & $\underline{0.46}$ & & & \\
\hline 128 & Impulsivity & DecidesQuicklyPursues & $\overline{0.45}$ & & & \\
\hline 74 & Impulsivity & MakesUpMindSuddenly & $\overline{0.44}$ & & & \\
\hline 79 & InhibCn & HardTimeWaitingTalkWhenExcited & $\underline{0.43}$ & & & \\
\hline 72 & Impulsivity & CallsOutAnswersEarly & $\underline{0.43}$ & & & \\
\hline 96 & HighPL & LikesRecklessPlay & $\overline{0.32}$ & & & \\
\hline 42 & Impulsivity & StopsAndThinks & $\underline{-0.70}$ & & & \\
\hline 40 & InhibCn & StopFromTooQuick & $\overline{-0.46}$ & & & \\
\hline 135 & InhibCn & PlanCarefully & -0.43 & & & \\
\hline 37 & Activity & LikesPhysicalActivity & & $\underline{0.77}$ & & \\
\hline 2 & Activity & PhysicallyActive & & $\underline{0.76}$ & & \\
\hline 102 & Activity & LikesActiveGames & & $\overline{0.75}$ & & \\
\hline 23 & Activity & SportOverTV & & $\overline{0.73}$ & & \\
\hline 43 & Activity & LikesRunning & & 0.72 & & \\
\hline 127 & Activity & LikesRunOutside & & $\overline{0.72}$ & & \\
\hline 21 & Activity & PrefersOutdoorPlay & & $\overline{0.65}$ & & \\
\hline 66 & Activity & Energetic & & $\overline{0.63}$ & & \\
\hline 46 & ActivCn & MakeSelfRunFastWhenTired & & $\overline{0.54}$ & & \\
\hline 115 & HighPL & EnjoysChase & & $\overline{0.50}$ & & \\
\hline 3 & HighPL & HighSlides & & $\underline{0.46}$ & & \\
\hline 149 & AttnFocus & DistractedInClass & & & $\underline{0.80}$ & \\
\hline 80 & AttnFocus & HardTimePayingAttention & & & $\underline{0.79}$ & \\
\hline 120 & AttnFocus & ToldToPayAttention & & & $\underline{0.76}$ & \\
\hline 82 & Impulsivity & TeachersTellsPayAttention & & & $\underline{0.76}$ & \\
\hline 78 & AttnFocus & HardTimeConcentratingActivity & & & 0.75 & \\
\hline 17 & AttnFocus & LooksAroundHomework & & & $\overline{0.66}$ & \\
\hline 7 & AttnFocus & EasilyDistractedStory & & & 0.59 & \\
\hline 89 & ActivCn & DifficultBoringAssignment & & & $\overline{0.58}$ & \\
\hline 93 & ActivCn & FunActivityInsteadOfHomework & & & $\underline{0.50}$ & \\
\hline 20 & ActivCn & MakesSelfDoHomework & & & $\overline{-0.43}$ & \\
\hline 94 & Anger & AngryWhenTaskDifficult & & & & $\underline{0.66}$ \\
\hline 87 & Anger & AngryWhenCannotFindSmth & & & & 0.64 \\
\hline 110 & Anger & TemperTantrums & & & & 0.62 \\
\hline 105 & Sooth & UpsetWhenAngryForFiveMins & & & & 0.59 \\
\hline 53 & Anger & AngryWhenToyTaken & & & & 0.54 \\
\hline 24 & Sadness & SadBrokenPlans & & & & $\underline{0.49}$ \\
\hline 100 & Sadness & FeelingsEasilyHurt & & & & 0.48 \\
\hline 154 & Discomfort & CriesWhenLittleHurt & & & & 0.48 \\
\hline 61 & Anger & AngryWhenAskedToStopPlayEarly & & & & 0.47 \\
\hline 31 & Sadness & SadWhenToldToDoSomething & & & & 0.46 \\
\hline 107 & Sadness & FeelDownWhenNotAccompishTask & & & & $\underline{0.46}$ \\
\hline 64 & Sooth & CriesMoreThanCoupleMins & & & & $\underline{0.46}$ \\
\hline 19 & Sooth & DifficultToSoothe & & & & 0.45 \\
\hline 146 & Anger & MadWhenProvoked & & & & 0.43 \\
\hline 35 & Sadness & CriesToyBroken & & & & 0.41 \\
\hline
\end{tabular}




\begin{tabular}{|c|c|c|c|c|c|c|c|c|c|c|c|}
\hline Item\# & Scale & Item Description & FearNeedles/Shy & Shyness & Affiliation & LikesStories & PerSen & Sadness & Fantasy & Fear & Assertiv \\
\hline 30 & Discomfort & CriesInjection & $\underline{0.93}$ & & & & & & & & \\
\hline 63 & Fear & ScaredInjectionsDr & $\overline{0.82}$ & & & & & & & & \\
\hline 55 & Shyness & ShyNewPeople & & 0.81 & & & & & & & \\
\hline 118 & Shyness & Shy & & 0.75 & & & & & & & \\
\hline 47 & Shyness & SelfConsciousAroundPeople & & $\overline{0.54}$ & & & & & & & \\
\hline 28 & ActivCn & HardSpeakingWhenScared & & $\overline{0.42}$ & & & & & & & \\
\hline 84 & Assertiv & FirstSpeakUp & & $-\overline{-0.53}$ & & & & & & & \\
\hline 59 & Shyness & SaySomethingStrangers & & -0.63 & & & & & & & \\
\hline 33 & Affiliation & GoodFriendEveryDay & & & 0.56 & & & & & & \\
\hline 148 & Affiliation & LikesFeelingClose & & & 0.55 & & & & & & \\
\hline 156 & Affiliation & LikesBeingWithOthers & & & $\underline{0.55}$ & & & & & & \\
\hline 106 & Affiliation & FriendsVeryImportant & & & $\overline{0.52}$ & & & & & & \\
\hline 18 & Affiliation & FriendsWithMany & & & 0.46 & & & & & & \\
\hline 129 & Affiliation & Confides & & & $\overline{0.44}$ & & & & & & \\
\hline 73 & LowPL & EnjoysLookingBooks & & & & $\underline{0.79}$ & & & & & \\
\hline 86 & LowPL & LikesQuietReading & & & & 0.78 & & & & & \\
\hline 54 & Fantasy & LikesMakeBelieveStories & & & & $\underline{0.63}$ & & & & & \\
\hline 109 & PerSen & NoticesSmallEnvironmentChanges & & & & & 0.61 & & & & \\
\hline 123 & PerSen & FeelSmoothRough & & & & & $\underline{0.58}$ & & & & \\
\hline 111 & PerSen & NoticesThingsOthersDoNot & & & & & $\underline{0.53}$ & & & & \\
\hline 77 & PerSen & TouchesSoftMaterial & & & & & 0.51 & & & & \\
\hline 114 & PerSen & NoticesSmallSpecksDirt & & & & & $\underline{0.47}$ & & & & \\
\hline 150 & PerSen & NoticesNewClothing & & & & & 0.43 & & & & \\
\hline 44 & PerSen & NoticesBirdSounds & & & & & $\overline{0.41}$ & & & & \\
\hline 69 & Discomfort & BotheredLoudScratchySounds & & & & & $\overline{0.40}$ & & & & \\
\hline 133 & Sadness & FrequentlySad & & & & & & $\underline{0.64}$ & & & \\
\hline 144 & Sadness & SadWhenOthersHappy & & & & & & $\overline{0.61}$ & & & \\
\hline 97 & Sadness & ToldByOthers ThatSad & & & & & & 0.58 & & & \\
\hline 27 & Sadness & DowncastNoReason & & & & & & $\overline{0.51}$ & & & \\
\hline 71 & Fantasy & EnjoysDrawingPictures & & & & & & & $\underline{0.57}$ & & \\
\hline 151 & Fantasy & LikesMakingThings & & & & & & & $\overline{0.55}$ & & \\
\hline 104 & Fantasy & BigImagination & & & & & & & 0.45 & & \\
\hline 75 & Fear & AfraidBurglars & & & & & & & & 0.58 & \\
\hline 140 & Fear & AfraidDark & & & & & & & & $\underline{0.58}$ & \\
\hline 153 & Fear & FrightenedNightmares & & & & & & & & $\overline{0.55}$ & \\
\hline 155 & Assertiv & EnjoysWinning Argument & & & & & & & & & 0.60 \\
\hline 122 & Assertiv & UsuallyWins Argument & & & & & & & & & 0.58 \\
\hline 131 & Assertiv & LikesBeingInCharge & & & & & & & & & 0.53 \\
\hline 98 & Assertiv & ChoosesGamesWithOthers & & & & & & & & & 0.51 \\
\hline
\end{tabular}

Note. Loadings less than $|.40|$ are not included; one lower-order factor had a single item loading on it, i.e., "Gets angry when s/he makes a mistake" which also loaded significantly on another factor; as a result, this scale was excluded from higher-order exploratory factor analysis results in a total of 13 lower-order factors extracted; ActivCn = Activation Control; Activity = Activity Level; Anger = Anger/Frustration; Assertiv. = Assertiveness/Dominance; AttnFocus = Attentional Focusing; Fantasy = Fantasy/Openness; HighPL = High Intensity Pleasure; InhibCn = Inhibitory Control; LowPL = Low Intensity Pleasure; PerSen = Perceptual Sensitivity; Sooth = Soothability/Falling Reactivity; Ang/Sad = Anger/Sadness; FearNeedles/Shy = Fear of Needles/Shyness 
Table 4.

Bivariate Associations between the 17 Original TMCQ scales and the 13 New Lower-Order Factors

\begin{tabular}{|c|c|c|c|c|c|c|c|c|c|c|c|c|c|c|c|}
\hline 17 & \multicolumn{15}{|c|}{13 New Lower-Order Factors } \\
\hline $\begin{array}{l}\text { TMCQ } \\
\text { scales }\end{array}$ & Imp & Activ & $\begin{array}{l}\text { Low } \\
\text { Attn } \\
\text { Foc }\end{array}$ & $\begin{array}{l}\text { Ang/ } \\
\text { Sad }\end{array}$ & $\begin{array}{l}\text { Fear } \\
\text { Needles/ } \\
\text { Shy }\end{array}$ & Shy & Affil & $\begin{array}{l}\text { Likes } \\
\text { Stories }\end{array}$ & PerSen & Sad & Fant & Fear & Assert & $M$ & $S D$ \\
\hline ActivCn & $-.49 * *$ & $.37 * *$ & $-.65 * *$ & $-.53 * *$ & $-.16^{*}$ & $-.39 * *$ & $.25 * *$ & $.24 * *$ & .04 & $-.42 * *$ & $.19 * *$ & $-.24 * *$ & .03 & 3.53 & .50 \\
\hline Activ & $.12 * *$ & $.96 * *$ & -.02 & $-.14 * *$ & $-.12 * *$ & $-.18 * *$ & $.31 * *$ & -.04 & $.11 * *$ & $-.21 * *$ & .07 & -.08 & $.10^{*}$ & 3.93 & .68 \\
\hline Affil & -.05 & $.31 * *$ & $-.15 * *$ & $-.14 * *$ & -.01 & $-.32 * *$ & $.92 * *$ & $.23 * *$ & $.21 * *$ & $-.24 * *$ & $.33 * *$ & -.02 & .20 & 4.19 & .48 \\
\hline Anger & $.52 * *$ & $-.11 * *$ & $.42 * *$ & $.89 * *$ & $.14 * *$ & $.22 * *$ & -.02 & $-.15^{* *}$ & $.14^{* *}$ & $.46^{* *} *$ & -.05 & $.26 * *$ & $.27 * *$ & 2.77 & .73 \\
\hline Assert & $.22 * *$ & $.24 * *$ & -.06 & .06 & -.02 & $-.40 * *$ & $.39 * *$ & $.17 * *$ & $.15 * *$ & -.06 & $.18 * *$ & -.05 & $.88 * *$ & 3.58 & .51 \\
\hline AttnFoc & $-.75 * *$ & .07 & $-.97 * *$ & $.41 * *$ & -.05 & -.05 & .03 & $.35^{* *}$ & -.01 & $-.35 * *$ & $.11 * *$ & $-.20 * *$ & $-.08 *$ & 3.54 & .89 \\
\hline Discomf & $.27 * *$ & $-.26 * *$ & $.27 * *$ & $.62 * *$ & $.51 * *$ & $.24 * *$ & -.01 & -.04 & $.34 * *$ & $.46^{* *}$ & .05 & $.45 * *$ & $.10 * *$ & 2.38 & .60 \\
\hline Fant & -.07 & $.13 * *$ & $-.18 * *$ & -.01 & $.09 *$ & -.06 & $.34 * *$ & $.52 * *$ & $.30 * *$ & $-.10 * *$ & $.83 * *$ & -.01 & $.21 * *$ & 4.08 & .53 \\
\hline Fear & $.17 * *$ & $-.21 * *$ & $.20 * *$ & $.41 * *$ & $.52 * *$ & $.28 * *$ & -.07 & -.05 & $.23 * *$ & $.33 * *$ & .04 & $.82 * *$ & .01 & 2.43 & .68 \\
\hline HighPL & $.34 * *$ & $.61 * *$ & $.14 * *$ & .02 & $-.13 * *$ & $-.27 * *$ & $.26 * *$ & -.01 & $.12 * *$ & -.06 & .09 & $-.18 * *$ & $.31 * *$ & 3.40 & .58 \\
\hline $\operatorname{Imp}$ & $.98 * *$ & .02 & $.71 * *$ & $.50 * *$ & .04 & -.04 & .06 & $-.21 * *$ & $.10 * *$ & $.32 * *$ & -.06 & $.20 * *$ & $.28 * *$ & 2.62 & .69 \\
\hline InhibCn & $-.82 * *$ & $.10 *$ & $-.66 * *$ & $-.38 * *$ & -.01 & .01 & .06 & $.29 * *$ & .04 & $-.28 * *$ & $.18 * *$ & $-.16^{* *}$ & $-.14 * *$ & 3.44 & .60 \\
\hline LowPL & $-.14 * *$ & $.13 * *$ & $-.29 * *$ & -.05 & .07 & -.05 & $.28 * *$ & $.73 * *$ & $.39 * *$ & $-.09 *$ & $.38 * *$ & -.06 & $.16^{* * *}$ & 3.64 & .55 \\
\hline PerSen & -.01 & $.22 * *$ & $-.15 * *$ & $.09 *$ & .03 & .02 & $.25 * *$ & $.18 * *$ & $.92 * *$ & .05 & $.30 * *$ & .07 & .11 & 3.35 & .59 \\
\hline Sad & $.36 * *$ & $-.20 * *$ & $.36 * *$ & $.85 * *$ & $.23 * *$ & $.40 * *$ & .03 & $-.10 *$ & $.27 * *$ & $.77 * *$ & -.01 & $.36 * *$ & $.11 *$ & 2.36 & .55 \\
\hline Shy & -.01 & $-.17 * *$ & $.08 *$ & $.35 * *$ & $.13 * *$ & $.95 * *$ & $-.23 * *$ & -.03 & $.13 * *$ & $.39 * *$ & -.01 & $.22 * *$ & $-.11 * *$ & 2.36 & .82 \\
\hline Sooth & $-.45 * *$ & $.21 * *$ & $-.45 * *$ & $.79 * *$ & $-.19 * *$ & $-.35 * *$ & $.10 *$ & $.15 * *$ & $-.18 * *$ & $-.62 * *$ & $.10 * *$ & $-.38 * *$ & $-.08 *$ & 3.83 & .64 \\
\hline$M$ & 2.56 & 3.98 & 2.51 & 2.71 & 2.90 & 2.50 & 3.98 & 4.06 & 3.05 & 1.57 & 4.28 & 2.38 & 3.35 & & \\
\hline$S D$ & .68 & .66 & .86 & .64 & 1.24 & .79 & .60 & .83 & .64 & .61 & .69 & .95 & .64 & & \\
\hline
\end{tabular}

Note. ${ }^{* *} p<.01 ; * p<.05 ;$ ActivCn = Activation Control; Activ = Activity Level; Anger = Anger/Frustration; Assert =

Assertiveness/Dominance; AttnFoc $=$ Attentional Focusing; Fant = Fantasy/Openness; HighPL = High Intensity Pleasure; InhibCn = Inhibitory Control; LowPL = Low Intensity Pleasure; PerSen = Perceptual Sensitivity; Sooth = Soothability/Falling Reactivity;

Ang/Sad = Anger/Sadness; FearNeedles/Shy = Fear of Needles/Shyness; Shy=Shyness; Affil=Affiliation; Sad=Sadness;

Imp=Impulsivity; Discomf=Discomfort 
Table 5.

Higher-Order Exploratory Factor Analyses of the TMCQ Lower-Order Scales

\begin{tabular}{lrrr}
\hline Lower-Order Factors & Factor 1 & Factor 2 & Factor 3 \\
\hline Impulsivity & $\mathbf{0 . 9 4}$ & -0.08 & 0.00 \\
Activity & -0.01 & $\mathbf{- 0 . 3 2}$ & $\mathbf{0 . 3 1}$ \\
Low Attentional Focus & $\mathbf{0 . 8 0}$ & 0.02 & -0.28 \\
Anger/Sadness & $\mathbf{0 . 4 9}$ & $\mathbf{0 . 5 8}$ & 0.03 \\
Fear of Needles/Shyness & 0.01 & $\mathbf{0 . 3 2}$ & 0.08 \\
Shyness & -0.04 & $\mathbf{0 . 5 6}$ & -0.24 \\
Affiliation & 0.00 & -0.13 & $\mathbf{0 . 5 7}$ \\
Likes Stories & $\mathbf{- 0 . 3 4}$ & 0.04 & $\mathbf{0 . 4 3}$ \\
Perceptual Sensitivity & 0.01 & 0.27 & $\mathbf{0 . 3 9}$ \\
Sadness & $\mathbf{0 . 3 5}$ & $\mathbf{0 . 5 6}$ & -0.12 \\
Fantasy/Openness & -0.17 & 0.05 & $\mathbf{0 . 4 8}$ \\
Fear & 0.19 & $\mathbf{0 . 3 9}$ & 0.00 \\
Assertiveness/Dominance & 0.23 & -0.04 & $\mathbf{0 . 4 2}$ \\
\hline
\end{tabular}

Note. Primary loadings $\geq .40$ are bolded; loadings $\geq .30$ are bolded and italicized; Factor 1

correlated with Factors 2, 3, at .13 and .17 respectively; Factor 2 correlated with Factor 3 at .01. 
Table 6.

Tests of Structural Invariance across the Two Samples (ON and NY): Three-Factor Model

\begin{tabular}{|c|c|c|c|c|c|c|}
\hline Model Tested & $d f$ & RMSEA & $\triangle \mathrm{RMSEA}$ & CFI & $\Delta \mathrm{CFI}$ & PASS? \\
\hline Configural invariance & 84 & .068 & & .931 & & YES \\
\hline Weak invariance & 114 & .059 & .009 & .929 & .002 & YES \\
\hline Strong invariance & 127 & .069 & .010 & .892 & .037 & YES \\
\hline Strict Invariance & 140 & .068 & .001 & .883 & .009 & YES \\
\hline
\end{tabular}




\section{The Structure of Observed Temperament in Preschoolers}

Individual differences in reactivity and self-regulation are conceptualized as temperament (Rothbart, 1981). Decades of research provide evidence that temperament is stable across time (Caspi, 2000; Durbin, Hayden, Klein, \& Olino, 2007; Eisenberg, Guthrie, Murphy, Shepard, Cumberland, \& Carlo, 1999; Eisenberg, Valiente, Spinrad, Cumberland, Liew, Reiser et al., 2009; Kochanska, Murray, \& Coy, 1997; Laursen, Pulkkinen, \& Adams, 2002; Pesonen, Raikkonen, Keskivaara, \& Keltikangas-Jarvinen, 2003; Shiner, Masten, \& Tellegen, 2002). Extant literature on temperament in young children links this construct to important outcomes later in life, such as emerging symptoms of internalizing and externalizing psychopathology

(e.g., Kochanska \& Knaack, 2003; Kotelnikova, Mackrell, Jordan, \& Hayden, 2015; Nigg, 2006; Nigg, Goldsmith, \& Sachek, 2004), obesity (e.g., Wu, Dixon, Dalton, Tudiver, \& Liu, 2011), social competence (Spinrad et al., 2007), and career stability (e.g., Blatný, Millová, Jelínek, \& Osecká, 2015).

Nevertheless, important questions regarding the nature and structure of early temperament remain unanswered. In particular, although the predictive value of temperament for important outcomes has been demonstrated, there is a lack of consensus concerning the structure of child temperament. Several structural models of child temperament have been developed (e.g., Abe \& Izard, 1999; Abe, 2005; Buss \& Plomin, 1984; Caspi \& Shiner, 2006; Halverson, Havil, Deal, Baker, Victor, Povopoulos, et al., 2003; Lamb, Chung, Wessels, Broberg, \& Hwang, 2002; Rothbart, Ahadi, Hershey, \& Fisher, 2001; Simonds \& Rothbart, 2004; Thomas \& Chess, 1977; Zentner \& Bates, 2008); however, these models are predominantly grounded in theory rather than empirical findings, and differ widely on the subject of the nature and number of primary dimensions required to account for children's individual differences (De Pauw, Mervielde \& 
Leeuwan, 2009; De Pauw \& Mervielde, 2010).

Regarding the specifics of these models, Thomas and Chess (1977) proposed a model of child temperament comprised of nine bipolar dimensions, including activity, approach/withdrawal, adaptability, mood, threshold of responsiveness/sensitivity, intensity of reaction, distractibility, rhythmicity/regularity, and attention span/task persistence. More recently, Rothbart and colleagues (2001) described a three-factor model of temperament in early childhood that has guided much contemporary work. This model includes Negative Affectivity (NA; consisting of fear, anger, sadness, discomfort, and low soothability), Extraversion/Surgency (including facets of activity, impulsivity, sociability, assertiveness, and high intensity pleasure), and Effortful Control (EC; including facets of attentional focusing, inhibitory control, low intensity pleasure, and perceptual sensitivity). Simonds and Rothbart (2004) reported evidence supporting the emergence of a fourth higher-order factor later in childhood, Sociability/Affiliation, comprised of affiliation and fantasy/openness scales. Finally, a growing body of research (e.g., Barbaranelli, Caprara, Rabasca, \& Pastorelli, 2003; John, Caspi, Robins, Moffit, \& Stouthamer-Loeber, 1994; Tackett, Slobodskaya, Mar, Deal, Halverson, Baker et al., 2012) supports the validity of the five-factor taxonomy of adult personality (i.e., the five-factor model or FFM consisting of Extraversion, Neuroticism, Conscientiousness, Agreeableness, and Openness to Experience; McCrae \& Costa, 1997) in characterizing child temperament.

With the exception of the FFM, existing structural models applied to child temperament were developed using a purely theory-driven, top-down approach, such that the higher-order structure was determined by expert consensus. While the higher-order structure of some of these measures has been replicated via factor analyses at the scale level (e.g., Ahadi, Rothbart, \& Ye, 
1993; Kochanska, De Vet, Goldman, Murray, \& Putnam, 1994; Richard, Davis, \& Bums, 2008), lower- and higher-order tests of these models are rare, likely due to the large sample sizes needed for such work. The few extant factor-analytic studies of Thomas and Chess' nine-factor model conducted at the item level did not replicate the nine bipolar dimensions (Martin, Wisenbaker, and Huttunen, 1994; Presley \& Martin, 1994), nor have such methods provided support for Rothbart's three- or four-factor models (Kotelnikova, Olino, Klein, Kryski, \& Hayden, 2016; Kotelnikova, Olino, Klein, Mackrell, \& Hayden, 2016). Thus, more work aimed at identifying the primary dimensions of child temperament is needed.

The development of the FFM differs from the aforementioned models in that it was developed using an atheoretical, lexical approach, and has been extensively validated in adults and adolescents across diverse cultures ( McCrae \& Terracciano, 2005; McCrae et al., 2010). However, some researchers have been unable to recover the full FFM in younger children (e.g., Abe \& Izard, 1999; De Pauw et al., 2009; Lamb et al., 2002; Mervielde, Buyust, \& De Fruyt, 1995), and the evidence for the validity of this model is stronger for older children and adolescents relative to younger children. For example, evidence suggests that additional higherorder factors (e.g., activity) beyond the Big Five are needed to capture meaningful variance in child temperament (e.g., Abe \& Izard, 1999; De Pauw et al., 2009; Lamb et al., 2002; Shiner \& DeYoung, 2013). Further, Openness to Experience has not been consistently recovered in studies of child temperament/personality using parent reports (e.g., DePauw et al., 2009). Thus, despite its extensive support in older samples, the FFM may not adequately capture the structure of child temperament.

From a methodological standpoint, it is noteworthy that extant work on the structure of child temperament has relied almost exclusively on parent-report measures. Parent-report 
measures of child temperament are widely used by researchers due to their affordability, efficiency, and potential to capitalize on caregivers' extensive knowledge of their child's behavior across time and multiple contexts (Pauli-Pott, Mertesacker, Bade, Haverkock, \& Beckmann, 2003; Rothbart et al., 2001; Rothbart \& Bates, 2006). However, caregiver reports have typically shown low convergent validity with other assessment approaches (Durbin et al., 2007), and are influenced by caregiver personality, mood state, availability biases (Durbin et al., 2007; Durbin \& Wilson, 2012; Hayden, Durbin, Klein, \& Olino, 2010; Rothbart \& Bates, 2006), and caregivers' symptoms of psychopathology (e.g., maternal symptoms of anxiety and depression; Durbin \& Wilson, 2012). As such, the validity of caregiver-report measures has been questioned (e.g., Kagan, 1998).

Laboratory observational measures, the primary alternative approach, are expensive and time-consuming to collect, and capture only a narrow slice of child behavior at a given time point (Gagne, Van Hulle, Aksan, Essex, \& Goldsmith, 2011; Goldsmith, Reilly, Lemery, Longley, \& Prescott, 1995). Nevertheless, these have several advantages over caregiver reports, such as reducing rater bias through the use of standardized coding procedures and stimuli (e.g., Gagne et al., 2011; Goldsmith et al., 1995). This method also facilitates observation of child behaviors that occur relatively less frequently in naturalistic settings (Durbin et al., 2007; Durbin, 2010). The predictive validity of observational measures has been examined, with work establishing that such temperament indices show meaningful associations with children's psychophysiological systems (Fox, Henderson, Marshall, Nichols, \& Ghera, 2005; Mackrell et al., 2014), moral development (Kochanska, Murray, Jacques, Koenig, \& Vandegeest, 1996), and quality of parent-child relationships (Kochanska, Aksan, \& Carlson, 2005). Observed affective traits (i.e., positive and negative emotionality) in preschoolers have been linked to maternal 
mood disorders (Durbin, Klein, Hayden, Buckley, \& Moerk, 2005; Olino, Klein, Dyson, Rose, \& Durbin, 2010), EEG asymmetries (Shankman, Tenke, Bruder, Durbin, Hayden, \& Klein, 2005), serotonin transporter gene polymorphisms (Hayden, Dougherty, Maloney, Durbin, Olino, \& Nurnberger et al., 2007), and the development of depressotypic cognitions and emerging internalizing symptoms in middle childhood (Dougherty, Klein, Durbin, Hayden, \& Olino, 2010; Hayden, Klein, Durbin, \& Olino, 2006; Kotelnikova et al., 2015; Kotelnikova, Olino, Mackrell, Jordan, \& Hayden, 2013). Overall, observational assessments of temperament have demonstrated meaningful associations with both normal and abnormal processes, indicating their utility for developmental psychopathology research (Durbin, 2010).

Regardless of the relative merits of each approach, parent-report and observational measures typically yield largely unique information on child temperament, as agreement between these two methods is low (e.g., Durbin et al., 2007; Majdandzic, \& van den Boom, 2007). Given this low convergence, it may be the case that the two methods lead to different conclusions regarding the nature of the structure of child temperament. However, research on the structure of observed temperament in children is scarce, with only two studies addressing this issue. In the first of these, Dyson, Olino, Durbin, Goldsmith, and Klein (2012) used a combination of exploratory and confirmatory factor analytic techniques to derive a five-factor model of observed child temperament in 559 preschoolers. The final model had some similarities to the three-factor model proposed by Rothbart and colleagues (2001), in that factors reflecting Positive Affect (PA)/Interest, Dysphoria, and Impulsivity/Constraint were extracted, which resemble Extraversion/Surgency, NA, and EC in Rothbart's model (Rothbart et al., 2001). However, a fourth factor, Sociability, was also derived. Sociability is subsumed within the Extraversion/Surgency factor in Rothbart's model; it is also included in the higher-order 
Extraversion factor in empirically derived models of adult personality (i.e., the FFM; McCrae \& Costa, 1997). Dyson et al. (2012) were also unable to recover a factor that consisted of fear, anger, and sadness, although such a factor is described in most models of child and adult temperament/personality (e.g., McCrae \& Costa, 1997; Rothbart et al., 2001; Tellegen, 1985; Watson \& Clark, 1993). Instead, they recovered a Dysphoria factor consisting of anger, sadness, and hostility and a Fear/Behavioral Inhibition (BI) factor consisting of fear, BI, and clinginess. Dyson et al. (2012) concluded that some traits, such as sociability and PA/interest, as well as dysphoria and fear/inhibition, do not coalesce into the relevant higher-order factors (Extraversion and Neuroticism) until later in life.

In the only other extant study examining the primary dimensions of observed temperament in children, Kotelnikova and colleagues (2013) recovered a four-factor structure consisting of PA/Sociability, Disinhibition/Anger, Fear/BI, and Sadness in 205 7-year-olds. As in the observed temperament model of Dyson et al. (2012), different facets of NA (fear, anger, and sadness) loaded onto separate factors. However, unlike Dyson et al. (2012), we failed to extract a clear factor related to EC, although our Disinhibition/Anger factor subsumed some ECrelated traits, such as low compliance and impulsivity. Unfortunately, as developmentally appropriate tasks tapping EC in middle childhood have not been developed, we were unable to include such tasks. Thus, our failure to extract a clear EC factor could have resulted from the lack of relevant tasks in our protocol. Our model bore marginal resemblance to the structure proposed by Simonds and Rothbart (2004) for middle childhood, such that the observed PA/Sociability factor resembled Simonds and Rothbart's Extraversion/Surgency and Sociability/Affiliation factors combined; however, the remaining observed factors showed little resemblance to parent-reported NA and EC. 
In summary, while the conceptual overlap between the models reviewed here tends to be modest, each includes affective components (i.e., Mood, Extraversion/Surgency, Negative Affectivity, or Neuroticism) and at least one regulatory component (e.g., Effortful Control, Conscientiousness, or Attention Span/Task Persistence). However, further methodological work aimed at clarifying structure is badly needed, as the lack of consensus on a taxonomy of child temperament undoubtedly hampers progress in the field, particularly with regard to questions regarding the development of temperament over time. A comprehensive trait structure provides an organizational framework for the primary units of temperament/personality, allowing the integration of findings across research studies that may use different instruments to assess this construct (Shiner \& DeYoung, 2013). A unified taxonomy of child and adult temperament/personality traits would aid investigations of how traits develop across the lifespan, and which traits are particularly important for different adaptive and maladaptive outcomes (Shiner \& DeYoung, 2013), both short- and long-term.

Thus, in the present study, we aimed to extend the literature on the structure of child temperament by examining the primary dimensions of observed child temperament in a large sample of preschoolers $(N=409)$. As noted, other than Dyson et al. (2012) and Kotelnikova et al. (2013), we know of no other published research using standardized observational measures to characterize the structure of child temperament. Findings from these two studies suggest both similarities and differences in the structure of temperament in young children relative to adult temperament. However, data presented in these two studies were collected from samples of different ages (3-year-olds in Dyson et al., 2012 and 7-year-olds in Kotelnikova et al. 2013), and using different observational batteries of behavioral tasks. In the present study, we focus on the structure of preschoolers' observed temperament using a highly similar battery of tasks to those 
used by Dyson et al. (2012), expecting to find similarities to the models derived by Dyson et al. (2012) and perhaps Kotelnikova et al. (2013). For example, given the consistent evidence for higher-order traits resembling Extraversion/PA, Neuroticism/NA, and Constraint/EC vs. Impulsivity in the child temperament and adult personality literatures (Caspi \& Shiner, 2006; De Pauw \& Mervielde, 2010; Markon, Krueger, \& Watson, 2005), we anticipated to find dimensions reflecting these constructs.

The concurrent predictive validity of the model was addressed by examining associations between higher-order factors and symptoms of child psychopathology. As a key goal of temperament/personality research is to predict important behavior and outcomes, models that lack the capacity to do so are limited in value. In light of the extant literature on relating child temperament to psychopathology (e.g., Dougherty et al., 2010; Eisenberg et al., 2009; Hayden, Klein, \& Durbin, 2005; Kotelnikova et al., 2015; Tackett, 2006), we examined associations between observed temperament and internalizing (i.e., depressive and anxious) and externalizing child symptoms (i.e., attentional problems and aggressive behavior). Based on previous work implicating NA as a broad risk factor for psychopathology (e.g., Clark, 2005), and consistent with the literature relating parent-reported NA and child psychopathology, we expected to find significant associations between child temperament dimensions related to NA and children's internalizing (e.g., Dougherty et al., 2010; Eisenberg et al., 2009; Kagan, 1998; Kotelnikova et al., 2015) and externalizing symptoms (e.g., Kim, Walden, Harris, Karrass, \& Catron, 2007; Morris, Silk, Steinberg, Terranova, \& Kithakye, 2010; Tackett, Martel, \& Kushner, 2012). As per the tripartite model (e.g., Clark, 2005), we expected to see an association between lower levels of any PA-related dimensions that might emerge and children's symptoms of depression. 


\section{Method}

\section{Participants}

Participants were 409 3-year-old children (201 boys; 49\%; $\left.M_{\text {age }}=3.02 ; S D_{a g e}=.16\right)$ and their primary caregivers, recruited for a study of child temperament. Families were recruited through a university's research participant pool and advertisements placed in local daycares, recreational facilities, and websites. Children with significant medical or psychological problems were excluded from participation. Primary caregivers were mostly mothers $\left(N=380 ; 93 \% ; \mathrm{M}_{\text {age }}\right.$ $=33.53 ; S D=5.07)$. Family income varied widely $(5.5 \%<\$ 20,000 ; 11 \%=\$ 20,000-\$ 40,000$; $22.7 \%=\$ 40,001-\$ 70,000 ; 31.2 \%=\$ 70,001-\$ 100,000 ; 29.5 \%>\$ 100,001)$. Children were mostly Caucasian (90\%) and were of average cognitive ability $(M=112, S D=14.06$; PPVT; Dunn \& Dunn, 2007). During the lab visit, children completed a set of behavioral tasks and their primary caregivers completed a questionnaire package that included a measure of child internalizing and externalizing symptoms.

\section{Assessment of Temperament}

Children participated in 12 standardized developmentally appropriate laboratory tasks based on the Laboratory Temperament Assessment Battery (Lab-TAB; Goldsmith et al., 1995). A female experimenter administered these tasks. Tasks were designed to elicit a wide array of temperamental characteristics and were videotaped for coding purposes. Carryover effects were minimized by ensuring that no tasks meant to elicit comparable affective reactions occurred successively; children were also given a short opportunity to play in between each task to return to a baseline state. The complete assessment spanned a total of 1.5 to 2 hours. The child's primary caregiver was present in the main experimental area with his/her child for all episodes except Stranger Approach, Box Empty, and a portion of Pop-up Snakes. When in the room, the 
caregiver was instructed to work on questionnaires at a desk in the back corner of the room while avoiding interaction with the child. A description of each task is provided below.

Risk Room. The child and experimenter entered a room containing novel and ambiguous stimuli, including a small staircase, a mattress, a balance beam, a Halloween mask, a cloth tunnel, and a large, black cardboard box. The experimenter left for five minutes after instructing the child to play with the stimuli "however you like." When the experimenter returned, she asked the child to interact with each stimulus in the room. This episode was derived from methods developed by Kagan and colleagues (e.g., Kagan, 1998; Kagan, Reznick, \& Snidman, 1986).

Tower of Patience. The child and experimenter took turns building a tower with large blocks. During each of her turns, the experimenter adhered to a schedule of increasingly lengthy delays before placing her block on the tower (Kochanska et al., 1996).

Puzzle with Parent. Based on the Teaching Tasks battery (Egeland, Weinfield, Hiester, Lawrence, Pierce, \& Chippendale, 1995), the child and parent were seated at a table in the centre of the experimental room and given a difficult block puzzle to work on together for five minutes while the experimenter left them alone. Child and parent collaborated on the puzzle, which had six different solutions, until the experimenter returned. To enhance motivation to complete the puzzle, the dyad was told to place the pictures of their completed puzzles on one corner of their table so they could show the experimenter how many they were able to solve by the end of the episode.

Stranger Approach. The child was left alone in the experimental area under the premise that the experimenter needed to get a toy for further play. While the child was alone, an unfamiliar male research assistant entered the room and spoke to the child while slowly moving closer, following a standardized script and timed intervals. After asking the child four 
standardized questions, the stranger left and the main experimenter returned. The same stranger then returned, greeted the experimenter and child, and was introduced to the child as the experimenter's friend.

Car Go. The child and experimenter played with two remote controlled cars for several minutes. The experimenter and child raced their cars, with the experimenter allowing the child to win every time.

Transparent Box. The child selected a toy, which the experimenter locked in a transparent box. The child was then left to work to open the box with a set of keys that were, unbeknownst to the child, inoperable. After a few minutes, the experimenter returned with the correct key, and helped the child access the toy.

Pop-up Snakes. The experimenter showed the child what appeared to be a can of potato chips, actually containing coiled spring snakes. The experimenter demonstrated the trick, and encouraged the child to surprise his or her parent with the snakes.

Jumping Spider. The child and experimenter were seated at a table in the centre of the room when a research assistant brought in a terrarium with a fuzzy, fake, black spider and placed it on the table. The experimenter showed the spider to the child and asked the child to touch the spider; when the child's hand was close to the spider, the experimenter manipulated the spider using an attached wire, making it appear to jump. This was repeated for a total of four trials, with the experimenter trying to convince the child to touch the spider each time. At the end of the fourth trial, the experimenter showed the child that the spider was a toy.

Snack Delay. The child was instructed to wait for the experimenter to ring a bell before eating a bite of a snack. The experimenter adhered to a schedule of varied delays before ringing the bell. 
Impossibly Perfect Green Circles. The child was repeatedly asked to draw the perfect green circle on a large piece of paper. After each drawing, the experimenter mildly criticized each circle. After two minutes of criticism, the experimenter praised the child's circles.

Popping Bubbles. The child and experimenter played with a bubble-shooting toy for several minutes, during which time, the experimenter was enthusiastic and encouraging.

Box Empty. The child was given a gift-wrapped box, under the pretense that an appealing toy was inside. After a brief interval in which the child was left alone to discover that the box was empty, the experimenter returned with several small toys for the child to keep, explaining that she had forgotten to place the toys inside.

Video coding procedures. Video-recordings of the laboratory tasks were coded by trained graduate and undergraduate raters. Coding procedures followed those described in previous studies (e.g., Durbin et al., 2005, 2007). Different coding methods were used for the affective, behavioral, $\mathrm{BI}$, and $\mathrm{EC}$ variables. Of the $12 \mathrm{Lab}-\mathrm{TAB}$ tasks, affective traits were coded in all tasks, BI was coded in three tasks (Risk Room, Stranger Approach, and Jumping Spider), and EC was coded in two tasks (Tower of Patience and Snack Delay). Raters were trained by a graduate student master-coder to an intraclass correlation (mean ICC) with the master coder of .80. Once this standard was reached, periodic reliability checks were conducted on $15-20 \%$ of all recordings (i.e., $2-3$ of every 15 tasks were coded for reliability). Coders periodically met to review recordings and prevent observer drift.

Affective traits. Each instance of facial, bodily, and vocal PA, anger, sadness, and fear in each episode was rated on a three-point scale (low, moderate, and high). The number of instances of moderate and high behaviors were weighted to account for their greater intensity (e.g., $\mathrm{N}$ of moderate intensity smiles*2; $\mathrm{N}$ of high intensity vocal sadness*3). After weighting, the total 
numbers of low, moderate, and high intensity behaviors were summed separately within each channel (facial, bodily, vocal) across the 12 episodes, standardized and then summed across the three channels to obtain composite scores for PA $(\alpha=.88)$, anger $(\alpha=.82)$, sadness $(\alpha=.77)$, and fear $(\alpha=.61)$. Mean ICCs for PA $(N=15)$, sadness $(N=14)$, anger $(N=16)$, and fear $(N=14)$ were $.71, .63, .70$, and .67 , respectively.

Other traits. A single rating on a four- or five-point scale was made for each behavioral variable per episode. These ratings were based on all of the behaviors thought to be relevant to each dimension during that episode. Interest $(\alpha=.62$; mean $\mathrm{ICC}=.69, N=17)$ was based on how engaged the child appeared in play. Anticipatory PA $(\alpha=.64$, mean ICC $=.73, N=17)$ was based on the quality and quantity of PA that occurred in anticipation of positive events (e.g., while waiting to receive the gift in Box Empty). Activity $(\alpha=.74$, mean ICC $=.79, N=18)$ was based on the quantity and quality of movement during each episode as well as the amount of vigor exhibited in the manipulation of stimuli. Sociability $(\alpha=.82$, mean $\operatorname{ICC}=.85, N=18)$ was based on the quality and quantity of the child's attempts to engage and interact with the experimenter and the parent. Compliance $(\alpha=.75$, mean ICC $=.84, N=18)$ was based on the severity of deviation from instructions given by the experimenter, the persistence of the noncompliance, and the degree to which these behaviors were judged to reflect an intentional unwillingness to comply. Impulsivity $(\alpha=.76$, mean $\mathrm{ICC}=.74, N=18)$ ratings were based on the child's tendency to act or respond without reflection or hesitation.

BI micro-coding. Three tasks were coded for BI (Risk Room, Stranger Approach, and Jumping Spider); episodes were divided into 20-to-30-second intervals or "epochs" based on a coding system developed by Goldsmith et al. (1995). Specific behaviors were coded in each individual epoch to evaluate the presence and intensity of conventional BI behavioral responses. 
During the Risk Room episode, latencies to touch each specific object, latency to the child's first fear response and to the child's first verbalization were recorded, and latencies to comply with the experimenter's requests were recorded. In each 20-30-second epoch, bodily fear, tentative play, time spent playing, references to parent (e.g., child looks to parent), proximity to parent, fearful or wary questions or comments, and amount of time talking were coded on a three-point scale. In addition, noncompliance and references to experimenter (i.e., child looks to experimenter) were coded on four- and two-point scales, respectively.

In the Stranger Approach episode, bodily and vocal fear were coded during each epoch on a three-point scale using the same principles that were used for coding the Risk Room task. In addition to these behaviors, stilling/freezing was coded as the duration of time during which a child exhibited a marked decrease in activity. Approach, avoidance, gaze aversion, and verbal/nonverbal interaction were coded on a two- to three-point scale only for epochs in which the stranger was present. The latency from the time the experimenter said she would leave the room to the child's first fear response was also recorded, as was the latency from the time the stranger entered the room to the child's first vocalization.

The Jumping Spider episode was divided into four trials for coding purposes; each trial began when the experimenter told the child to "go ahead and pet the spider". Latency to the first definite fear response was recorded. In each trial, intensity of fear expression, intensity of bodily fear, intensity of vocal distress, intensity of bodily fear, approach, withdrawal, gaze aversion, and startle were coded on a two- to three-point scale. Presence or absence of play with spider was recorded based on the child's play with the spider, when provided the opportunity after the last trial.

Item analysis was used to create a composite BI scale, after reverse-coding as needed, 
standardizing, and aggregating the standardized items to derive a single BI variable comprised of the ratings from all three tasks. Therefore, the final BI scale consisted of an average of z-scored codes $(\alpha=.79 ;$ mean ICC $=.71, N=32)$ from the Risk Room, Stranger Approach, and Jumping Spider tasks.

EC coding. EC was coded in two tasks: Tower of Patience and Snack Delay. Each task was coded for failures to wait (i.e., placing a block out of turn during the tower task, or eating the candy before the bell was rung during the snack task) and child prompts to experimenter (i.e., child requesting that experimenter place a block or ring a bell immediately; see Carlson, 2005, Kochanska et al., 1996, and Kochanska, \& Knaack, 2003, for similar procedures). The number of failures to wait and child prompts to experimenter were summed for each task and then averaged again across tasks to create an aggregate inhibitory control scale $(\alpha=.76$; mean ICC $=.97, N=15)$.

\section{Assessment of Internalizing and Externalizing Symptoms}

During the lab visit, primary caregivers (93\% mothers) completed a measure of children's symptoms of psychopathology, the Child Behavior Checklist/4-18, (CBCL, Achenbach, 1991). The CBCL is a widely used measure designed to identify social, emotional, and behavioral problems in children, and was used as a measure of child psychopathology. The CBCL yields standard scores for eight empirically derived problem areas as well as three composite scores assessing overall Internalizing, Externalizing, and Total Problems (Achenbach, 1991). The internal consistencies of syndrome scales used in this study were moderate: withdrawn/depressed symptoms $(\alpha=.57)$, attentional problems $(\alpha=.61)$, and aggressive behavior problems $(\alpha=.86)$. Average scores for withdrawn/depressed symptoms, attentional problems, and aggressive behavior were low (see Table 4) and consistent with published means reported for a community sample (e.g., Rescorla, Bochicchio, Achenbach, Ivanova, Almqvist, 
Begovac et al., 2014).

\section{Statistical Approach}

To reduce skewness and kurtosis, $\log _{10}$ transformations were applied to the sadness and EC variables. These transformed variables were used in all of the analyses. Using an exploratory factor analytic framework, we evaluated three- to five-factor solutions. This range of factors was selected based on the extant literature and current theory regarding the number of factors needed to adequately characterize child and adult temperament/personality (e.g., Dyson et al., 2012; Kotelnikova et al., 2013; McCrae \& Costa, 1997; Rothbart et al., 2001; Simonds \& Rothbart, 2004; Watson \& Clark, 1993). Twelve observed temperament variables (i.e., PA, anticipatory PA, interest, activity, compliance, sociability, impulsivity, fear, anger, sadness, BI, and low EC)

were used in exploratory structural equation modeling (ESEM) using Mplus 7 statistical software (Muthen \& Muthen, 1998-2012). ESEM combines exploratory and confirmatory factor analytic techniques by allowing all observed indicators to load on all variables as well permitting posthoc model modifications (Asparouhov \& Muthen, 2009). For parameter estimation procedures, we used the maximum likelihood (ML) estimator (Muthen \& Muthen, 1998- 2012) and the geomin oblique rotation. Goodness-of-fit of ESEM solutions was evaluated using comparative fit index (CFI) values, of which those above .90 and .95 indicated acceptable and excellent fit (Hu \& Bentler, 1999), respectively. Root-mean-square of approximation (RMSEA) values that were lower than .05 indicated a close fit, and values up to 0.08 indicated acceptable fit (Marsh, Hau, \& Wen, 2004). Models that varied in terms of the number of factors were compared using the chisquare difference test.

To further improve model fit, model estimates and modification indices (MIs) were examined and post-hoc model fitting was conducted. We considered variables meaningful to a 
factor when their loadings were $\geq .40$, although those with loadings of $\geq .30$ were also considered in identifying and interpreting factors ${ }^{10}$. Factor scores were computed as sums of observed variables based on the structural analyses, and bivariate associations between these and the CBCL scales (withdrawn/depressed symptoms, attentional problems, and aggressive behavior) were examined. The goal of these analyses was to examine the concurrent predictive validity of the derived observed temperament factor scores.

\section{Results}

\section{Correlations between Major Study Variables}

Bivariate associations between all major study variables are presented in Table 1. There were no significant associations between child age and observed temperament. Family income was positively albeit weakly associated with sociability (see Table 1). Higher PPVT scores were associated with higher PA, anticipatory PA, interest, compliance, sociability, EC, and lower anger (see Table 1). PA showed strong positive associations with interest, activity, sociability, and anticipatory PA. Similarly, associations between impulsivity, anger, and low EC and compliance were strong. Although sadness, anger, and BI were moderately positively correlated, fear was uncorrelated with other facets of NA excluding a moderate positive association with BI. Bivariate associations between observed temperament variables and child sex showed that boys had higher levels of activity, impulsivity, sociability, interest, and anger, whereas girls showed greater compliance and EC (see Table 1). These results are consistent with previously reported sex differences in observed temperament (Olino, Durbin, Klein, Hayden, \& Dyson, 2013).

\footnotetext{
${ }^{10}$ Although a cut-off of .30 is sometimes used to designate an acceptable loading in exploratory factor analytic approaches, use of a more stringent cut-off of .40 is also common (Briggs \& MacCallum, 2003; Comrey, 1973; Hogarty, Kromrey, Ferron, \& Hines, 2004).
} 


\section{ESEM Results}

As noted previously, based on theory, we examined three- to five-factor solutions using ESEM. A three-factor solution had poor fit (RMSEA =.11; CFI =.92), while a four-factor solution had a significantly better fit $\left(\mathrm{RMSEA}=.07 ; \mathrm{CFI}=.98 ; \Delta \chi^{2}(9)=129.57 ; p<.001\right)$. A fivefactor model did not converge, so the four-factor solution was selected for further development. Based on modification indices, we added three covariance paths between sadness and anger, fear and anger, and compliance and interest. The final model, presented in Table 2 and Figure 1, was a good fit to the data $(\mathrm{RMSEA}=.05 ; \mathrm{CFI}=.99)$. PA, Anticipatory PA, activity and interest loaded on the first factor, labelled PA/Activity. Anger, low EC, impulsivity, compliance, and activity loaded on the second factor, Impulsivity/Anger. The third factor (Dysphoria) was comprised of fear, sadness, and BI, and the fourth factor (Surgency) was comprised of interest, sociability, and low EC. Correlations between higher-order factors (see Table $3 \&$ Figure 1) showed that PA/Activity was positively correlated with Surgency and negatively correlated with Dysphoria. Surgency was positively correlated with Impulsivity/Anger and negatively correlated with Dysphoria.

\section{Associations between Observed Temperament Factors and Child Symptoms of Psychopathology}

Observed temperament factor scores were computed based on the ESEM results, such that observed indicators with primary loadings of $\geq .40$ were $\mathrm{z}$-score transformed and summed up to derive the factor scores. Two indicators were allowed to retain their secondary loadings to ensure that each factor had at least three observed indicators (i.e., activity cross-loaded on PA/Activity and Impulsivity/Anger; low EC cross-loaded on Impulsivity/Anger and Surgency) ${ }^{11}$.

\footnotetext{
${ }^{11}$ A replication of the model was attempted in a different sample of preschoolers $(N=559)$ from Long Island, New York, USA via a confirmatory factor analytic approach. Results showed that the four-factor model reported here
} 
Bivariate associations between observed temperament factor scores and concurrent child withdrawn/depressed symptoms, attentional problems, and aggressive behaviour, as reported by primary caregivers, are presented in Table 4. PA/Activity showed a significant negative association with withdrawn/depressed symptoms and Dysphoria showed a significant positive association with these symptoms, which is consistent with the tripartite model (e.g., Clark, 2005). Both Impulsivity/Anger and Surgency were also negatively associated with withdrawn/depressed symptoms. The Impulsivity/Anger factor was associated with attentional problems and aggressive behavior, consistent with our prediction of associations between anger and externalizing problems (e.g., Kim et al., 2007; Morris et al., 2010; Tackett et al., 2012b).

\section{Discussion}

We extended the limited literature on the nature and structure of observed temperament in preschoolers, using ESEM to examine three- to five-factor solutions. While a five-factor model did not converge, and a three-factor model had a poor fit, a four-factor solution consisting of PA/Activity, Impulsivity/Anger, Dysphoria, and Surgency was a good fit to the data. Factor scores based on the model were meaningfully related to children's symptoms, thus demonstrating concurrent predictive validity.

Given that only one other study has investigated the structure of observed temperament in preschoolers, it is important to compare our findings to those of Dyson et al. (2012). Three of the four factors of our model (i.e., PA/Activity, Impulsivity/Anger, and Surgency) resemble those found by Dyson et al. (2012; i.e., PA/Interest, Constraint vs. Impulsivity, and Sociability). Although both models also include a factor labelled Dysphoria, its composition across the two studies is somewhat different: in the Dyson et al. (2012) study, this factor consisted of sadness 
and anger-related traits, while BI and fear formed a separate factor. In the present study, Dysphoria was comprised of sadness, fear, and BI variables, while anger did not have a strong loading on this factor. Thus, sadness was the only observed variable that loaded on the Dysphoria factor in both studies. While the reasons for this difference are unclear, Dyson et al. (2012) included several variables in their model that were not coded in our sample, and the presence of these additional indicators likely contributed to differences in our findings. Similarly, two of the twelve Lab-TAB tasks differed between the two studies. The current finding that anger loaded with impulsivity, activity, low compliance, and low EC rather than with other NA facets is consistent with conceptualizations of anger as an approach-related trait (Carver \& Harmon-Jones, 2009; Deater-Deckard, Beekman, Wang, Kim, Petrill, Thompson et al., 2010; Depue \& Collins, 1999; Eysenck \& Eysenck, 1985).

Our model included both PA-like and NA-like factors, and one factor comprised of EClike traits, consistent with several prominent models of child and adult temperament (e.g., Rothbart et al., 2001; Tellegen, 1985; Watson \& Clark, 1993). In particular, Rothbart's (2001) three-factor model of child temperament in preschoolers, which consists of Extraversion/Surgency, NA, and EC, bears resemblance to our derived model of observed temperament. Rothbart's Extraversion/Surgency factor includes scales tapping PA and activity level, consistent with the composition of the PA/Activity factor in our study. Similarly, there is overlap between Rothbart's EC factor and the observed Impulsivity/Anger factor in our study. However, there are also differences in the composition of our observed Impulsivity/Anger and Dysphoria factors and their counterparts in Rothbart's model. In particular, we found that anger did not load with other NA facets, while the NA factor proposed by Rothbart and colleagues (2001) is comprised of fear, anger, and sadness facets, consistent with adult models of trait NA 
(e.g., McCrae \& Costa, 1997; Tellegen, 1985; Watson \& Clark, 1993). However, recent factor analytic studies of Rothbart's model were not able to recover a single NA factor consisting of fear, anger, and sadness (Kotelnikova et al., 2016a \&b); thus, existing evidence for a single NA factor in early and middle childhood is not strong. Additionally, previous studies have identified shared genetic influences on impulsivity and anger (e.g., the T allele of the HTR2A 102T/C polymorphism; Anguelova, Benkelfat, \& Turecki, 2003; Arango, Huang, Underwood, \& Mann, 2003; Mann, Brent, \& Arango, 2001; Zalsman, Patya, Frisch, Ofek, Schapir, Blum, et al., 2011; Zhang, Ishigaki, Tani, Chen, Shih, Miyasato et al.,1997; the functional polymorphism of 30-bp upstream repeats in the promoter of the MAOA gene, i.e., MAOA-uVNTR; Du, Faludi, Palkovits, Sotonyi, Bakish, \& Hrdina, 2002). Given such evidence, it is not surprising that they clustered together in our study.

Although it has been argued that NA facets become increasingly intercorrelated over time (Dyson et al., 2012), Kotelnikova et al. (2013) found further differentiation of NA into three separate factors, consistent with other studies showing increased differentiation of this construct across development (Camras, Oster, Campos, Campos, Ujiie, Miyake et al., 1998; Izard, Fantauzzo, Castle, Haynes, Rayias, \& Putnam, 1995). To our knowledge, no study has investigated the underlying structure of observational measures of adult temperament. Thus, it is possible that such observational studies might reveal continued or increased differentiation between facets of NA in adulthood, which would differ substantially from models based on selfand parent-reports (e.g., McCrae \& Costa, 1997; Rothbart et al., 2001; Simonds \& Rothbart, 2004; Tellegen, 1985; Watson \& Clark, 1993). Such results would be consistent with recent behavior genetic findings (Clifford, Lemery-Chalfant, \& Goldsmith, 2015) indicating that observed fear, anger, and sadness are genetically distinct from each other. 
There are also similarities and differences between our four-factor model of observed temperament and the FFM (McCrae \& Costa, 1997). In particular, our model includes a reactive Extraversion-like factor (i.e., PA/Activity) and a Neuroticism-like factor (i.e., Dysphoria). Furthermore, a factor comprised of traits relevant to lower levels of Conscientiousness (i.e., Impulsivity/Anger) was obtained. The fourth factor of our model (Surgency) is characterized by approach-related traits (i.e., interest and sociability) and low levels of EC. This factor taps surgency, assertiveness, social dominance, and low compliance, and can be interpreted as a combination of Extraversion and low Agreeableness traits from the FFM (McCrae \& Costa, 1997). The Lab-TAB, our laboratory battery of behavioral tasks, was not designed to elicit variability in Openness to Experience, one of the five factors in the FFM (McCrae \& Terracciano, 2005; McCrae et al., 2010). However, this construct has also not been consistently recovered in studies of child temperament/personality using parent reports (e.g., DePauw et al., 2009), and the internal consistency of this factor in children younger than 6 years old is questionable (Caspi \& Shiner, 2006; Lamb et al., 2002). Developing age-appropriate tasks to assess this construct in preschoolers is an important area of further research, with the goal of clarifying whether it can validly assessed using such methods, and, if so, when it emerges during development.

We addressed the concurrent predictive validity of our observed temperament factors by examining their associations with parent-reported child symptoms. Overall, our findings converge with the extant literature based on parent reports of temperament and observational methods: higher levels of Impulsivity/Anger were associated with higher levels of child attentional problems and aggressive behavior reported by mothers, consistent with a literature suggesting that anger is an important contributor to externalizing problems (e.g., Kim et al., 
2007; Morris et al., 2010; Tackett et al., 2012b). Similar results were reported by Kotelnikova et al. (2013) in a study of observed temperament in 7-year-olds. We also found a positive association between PA/Activity and attentional problems which is unsurprising given that activity loaded together with PA and anticipatory PA, consistent with a number of accounts of the role of emotion and activity in childhood (Putnam, 2012). Finally, in accordance with the tripartite model (Clark \& Watson, 1999), there was a significant negative association between observed PA/Activity and withdrawn/depressed symptoms.

The present study has numerous strengths, including the use of observational measures of child temperament, which may have important advantages over informant-reports, including the use of standardized stimuli and coding procedures that minimize rater bias. Sophisticated factor analytic approaches were used to examine the structure of observed temperament in 409 preschoolers, contributing novel information to a small yet important literature. Achieving consensus on the nature and number of primary dimensions of child temperament is important toward developing a common language between personality and temperament researchers (Shiner \& DeYoung, 2013); the present study makes a significant contribution toward this goal.

However, it is also important to acknowledge several limitations of the present study. Although the observational method of assessing child temperament we used has numerous advantages, we cannot claim that we fully sampled all behaviors potentially relevant to child temperament. Specifically, the Lab-TAB (Goldsmith et al., 1995) was designed under the assumption that children's individual differences in emotion and self-regulation are core aspects of temperament. However, there could be other traits of importance that are either inadequately covered or elicited by the Lab-TAB, or were not included into our coding system. Like all factoranalytic methods, our structural analyses and conclusions about the nature of child temperament 
are constrained by the behaviors we included in our analyses. Secondly, internal consistency statistics and ICCs for the observed variables were low in some cases, albeit consistent with previous studies (e.g., Clifford et al., 2015; Durbin et al., 2007). Finally, testing the Lab-TAB and resulting models of observed child temperament in more ethnically diverse samples is an important area of future development.

The present study extends the small literature on the nature and structure of observed temperament in young children. We derived a four-factor model of observed temperament in preschoolers that was very similar to that described in the only other structural work on temperament in children this age (Dyson et al., 2012). Similarly, our model overlaps with extant models of child and adult temperament/personality (e.g., McCrae \& Costa, 1997; Rothbart et al., 2001; Simonds \& Rothbart, 2004; Tellegen, 1985; Watson \& Clark, 1993). Finally, the observed temperament factors showed meaningful associations with concurrent internalizing and externalizing child symptoms, providing further support for using observational methods in studies of child temperament. 


\section{References}

Abe, J. A. A. (2005). The predictive validity of the five-factor model of personality with preschool age children: A nine year follow-up study. Journal of Research in Personality, 39, 423-442. doi: 10.1016/j.jrp.2004.05.002

Abe, J. A. A., \& Izard, C. E. (1999). A longitudinal study of emotion expression and personality relations in early development. Journal of Personality and Social Psychology, 77, 566577. doi: $10.1037 / 0022-3514.77 .3 .566$

Achenbach, T.M. (1991). Manual for the Child Behavior Checklist/4-18 and 1991 profile. Burlington: University of Vermont, Department of Psychiatry.

Ahadi, S. A., Rothbart, M. K., \& Ye, R. (1993). Children's temperament in the US and China: Similarities and differences. European Journal of Personality, 7, 359-377. doi:10.1002/per.2410070506

Anguelova, M., Benkelfat, C., \& Turecki, G. (2003). A systematic review of association studies investigating genes coding for serotonin receptors and the serotonin transporter: I. affective disorders. Molecular Psychiatry, 8, 574-591. doi:10.1038/sj.mp.4001328

Arango, V., Huang, Y., Underwood, M. D., \& Mann, J. J. (2003). Genetics of the serotonergic system in suicidal behavior. Journal of Psychiatric Research, 37, 375-386. doi:10.1016/S0022-3956(03)00048-7

Asparouhov, T., \& Muthén, B. (2009). Exploratory structural equation modeling. Structural Equation Modeling, 16, 397-438. doi:10.1080/10705510903008204

Barbaranelli, C., Caprara, G. V., Rabasca, A., \& Pastorelli, C. (2003). A questionnaire for measuring the big five in late childhood. Personality and Individual Differences, 34, 645664. doi:10.1016/S0191-8869(02)00051-X 
Blatný, M., Millová, K., Jelínek, M., \& Osecká, T. (2015). Personality predictors of successful development: Toddler temperament and adolescent personality traits predict well-being and career stability in middle adulthood. PLOS ONE, 10.

Briggs, N. E., \& MacCallum, R. C. (2003). Recovery of weak common factors by maximum likelihood and ordinary least squares estimation. Multivariate Behavioral Research, 38, 25-56. doi:10.1207/S15327906MBR3801_2

Buss, A. H. \& Plomin, R. (1984). Temperament: Early developing personality traits. Hillside, NJ: Erlbaum.

Camras, L. A., Oster, H., Campos, J., Campos, R., Ujiie, T., Miyake, K., Wang, Lei, \& Meng, Z. (1998). Production of emotional facial expressions in European, American, Japanese, and Chinese infants. Developmental Psychology, 34, 616-628. doi:10.1037/00121649.34.4.616

Carlson, S. M. (2005). Developmentally sensitive measures of executive function in preschool children. Developmental Neuropsychology, 28, 595-616. doi:10.1207/ s15326942dn2802_3

Carver, C.S. \& Harmon-Jones, E. (2009). Anger is an approach-related affect: Evidence and implications. Psychological Bulletin, 135, 183-204. doi:10.1037/a0013965

Caspi, A. (2000). The child is the father of the man: Personality continuities from childhood to adulthood. Journal of Personality and Social Psychology, 78, 158-172.

Caspi, A., \& Shiner, R. L. (2006). Personality development. Hoboken, NJ, US: John Wiley \& Sons Inc, Hoboken, NJ.

Clark, L. A. (2005). Temperament as a unifying basis for personality and psychopathology. Journal of Abnormal Psychology, 114, 505-521. doi:10.1037/0021- 
843X.114.4.505

Clark, L. A., \& Watson, D. (1999). In Pervin L. A., John O. P. (Eds.), Temperament: A new paradigm for trait psychology. New York, NY, US: Guilford Press, New York, NY.

Clifford, S., Lemery-Chalfant, K., \& Goldsmith, H. H. (2015). The unique and shared genetic and environmental contributions to fear, anger, and sadness in childhood. Child Development, doi:10.1111/cdev.12394

Comrey, A.L. (1973). A first course in factor analysis. New York: Academic Press.

Deater-Deckard, K., Beekman, C., Wang, Z., Kim, J., Petrill, S., Thompson, L., \& DeThorne, L. (2010). Approach/positive anticipation, frustration/anger, and overt aggression in childhood. Journal of personality, 78, 991-1010.

De Pauw, S. S. W., \& Mervielde, I. (2010). Temperament, personality and developmental psychopathology: A review based on the conceptual dimensions underlying childhood traits. Child Psychiatry and Human Development, 41, 313-329. doi:10.1007/s10578-009$0171-8$

De Pauw, S. S. W., Mervielde, I., \& Van Leeuwen, K. G. (2009). How are traits related to problem behavior in preschoolers? similarities and contrasts between temperament and personality. Journal of Abnormal Child Psychology,37, 309-325. doi: 10.1007/s10802008-9290-0

Depue, R. A., \& Collins, P. F. (1999). Neurobiology of the structure of personality: Dopamine, facilitation of incentive motivation, and extraversion. Behavioral and Brain Sciences, 22, 491-569. doi: 10.1017/S0140525X99002046

Dougherty, L. R., Klein, D. N., Durbin, C. E., Hayden, E. P., \& Olino, T. M. (2010). Temperamental positive and negative emotionality and children's depressive symptoms: 
A longitudinal prospective study from age three to age ten. Journal of Social and Clinical Psychology, 29, 462-488. doi:10.1521/jscp.2010.29.4.462

Du, L., Faludi, G., Palkovits, M., Sotonyi, P., Bakish, D., \& Hrdina, P. D. (2002). High activityrelated allele of MAO-A gene associated with depressed suicide in males. NeuroReport: For Rapid Communication of Neuroscience Research, 13, 1195-1198.

doi:10.1097/00001756-200207020-00025

Dunn, L. M., \& Dunn, L. M. (2007). Peabody Picture Vocabulary Test (4 $^{\text {th }}$ ed.). Circle Pines, MN: American Guidance Service.

Durbin, C. E. (2010). Modeling temperamental risk for depression using developmentally sensitive laboratory paradigms. Child Development Perspectives, 4, 168-173. doi:10.1111/j.1750-8606.2010.00137.x

Durbin, C. E., Hayden, E. P., Klein, D. N., \& Olino, T. M. (2007). Stability of laboratoryassessed temperamental emotionality traits from ages 3 to 7 . Emotion, 7, 388-399. doi:10.1037/1528-3542.7.2.388

Durbin, C. E., Klein, D. N., Hayden, E. P., Buckley, M. E., \& Moerk, K. C. (2005). Temperamental emotionality in preschoolers and parental mood disorders. Journal of Abnormal Psychology, 114, 28-37. doi:10.1037/0021-843X.114.1.28

Durbin, C. E., \& Wilson, S. (2012). Convergent validity of and bias in maternal reports of child emotion. Psychological Assessment, 24, 647-660. doi:10.1037/a0026607

Dyson, M.W., Olino, T.M., Durbin, C.E., Goldsmith, H.H., \& Klein, D. N. (2012). The structure of temperament in preschoolers: A two-stage factor analytic approach. Emotion, 12, 4457.

Egeland B, Weinfield N, Hiester M, Lawrence C, Pierce S, \& Chippendale K. (1995). Teaching 
tasks administration and scoring manual. University of Minnesota.

Eisenberg, N., Guthrie, I. K., Murphy, B. C., Shepard, S. A., Cumberland, A., \& Carlo, G. (1999). Consistency and development of prosocial dispositions: A longitudinal study. Child Development, 70, 1360-1372.

Eisenberg, N., Valiente, C., Spinrad, T. L., Cumberland, A., Liew, J., Reiser, M., . . Losoya, S. H. (2009). Longitudinal relations of children's effortful control, impulsivity, and negative emotionality to their externalizing, internalizing, and co-occurring behavior problems. Developmental Psychology, 45, 988-1008. doi: 10.1037/a0016213

Eysenck, H. J., \& Eysenck, S. B. G. (1985). Personality and individual differences. New York: Plenum.

Fox, N. A., Henderson, H. A., Marshall, P. J., Nichols, K. E., \& Ghera, M. M. (2005). Behavioral inhibition: Linking biology and behavior within a developmental framework. Annual Review of Psychology, 56, 235-262. doi:10.1146/annurev.psych. 55.090902 .141532

Gagne, J. R., Van Hulle, C. A., Aksan, N., Essex, M. J., \& Goldsmith, H. H. (2011). Deriving childhood temperament measures from emotion-eliciting behavioral episodes: Scale construction and initial validation. Psychological Assessment, 23, 337-353. doi:10.1037/a0021746

Goldsmith, H. H., Reilly, J., Lemery, K. S., Longley, S., \& Prescott, A. (1995). Laboratory Temperament Assessment Battery: Preschool version. Unpublished manuscript.

Halverson, C. F., Havill, V. L., Deal, J., Baker, S. R., Victor, J. B., Pavlopoulous, V., ... Wen, L. (2003). Personality structure as derived from parental ratings of free descriptions of 
children: The inventory of child individual differences. Journal of Personality, 71, 9951026. doi: $10.1111 / 1467-6494.7106005$

Hayden, E. P., Dougherty, L. R., Maloney, B., Durbin, C. E., Olino, T. M., Nurnberger, J. I., Jr., . .. Klein, D. N. (2007). Temperamental fearfulness in childhood and the serotonin transporter promoter region polymorphism: A multimethod association study. Psychiatric Genetics, 17, 135-142. doi:10.1097/YPG.0b013e3280147847

Hayden, E. P., Durbin, C. E., Klein, D. N., \& Olino, T. M. (2010). Maternal personality influences the relationship between maternal reports and laboratory measures of child temperament. Journal of Personality Assessment, 92, 586-593. doi:10.1080/ 00223891.2010 .513308

Hayden, E. P., Klein, D. N., \& Durbin, C. E. (2005). Parent reports and laboratory assessments of child temperament: A comparison of their associations with risk for depression and externalizing disorders. Journal of Psychopathology and Behavioral Assessment, 27, 89100. doi:10.1007/s10862-005-5383-Z

Hayden, E. P., Klein, D. N., Durbin, C. E., \& Olino, T. M. (2006). Positive emotionality at age 3 predicts cognitive styles in 7-year-old children. Development and Psychopathology, 18, 409-423. doi: 10.1017/S0954579406060226

Hogarty, K. Y., Kromrey, J. D., Ferron, J. M., \& Hines, C. V. (2004). Selection of variables in exploratory factor analysis: An empirical comparison of a stepwise and traditional approach. Psychometrika, 69, 593-611. doi:10.1007/BF02289857

Hu, L., \& Bentler, P. M. (1999). Cutoff criteria for fit indexes in covariance structure analysis: Conventional criteria versus new alternatives. Structural Equation Modeling, 6, 1-55. doi:10.1080/10705519909540118 
Izard, C. E., Fantauzzo, C. A., Castle, J. M., Haynes, O. M., Rayias, M. F., \& Putnam, P. H. (1995). The ontogeny and significance of infants' facial expressions in the first 9 months of life. Developmental Psychology, 31, 997-1013. doi:10.1037/0012-1649.31.6.997

John, O. P., Caspi, A., Robins, R. W., Moffitt, T. E., \& Stouthamer-Loeber, M. (1994). The "little five": Exploring the nomological network of the five-factor model of personality in adolescent boys. Child Development, 65, 160-178.

Kagan, J. (1998). In Eisenberg N. (Ed.), Biology and the child. Hoboken, NJ, US: John Wiley \& Sons Inc, Hoboken, NJ.

Kagan, J., Reznick, J. S., \& Snidman, N. (1986). Temperamental inhibition in early childhood. The study of temperament: Changes, continuities and challenges, 53-67.

Kim, G., Walden, T., Harris, V., Karrass, J., \& Catron, T. (2007). Positive emotion, negative emotion, and emotion control in the externalizing problems of school-aged children. Child psychiatry and human development, 37, 221-239, doi: 10.1007/s10578006-0031-8

Kochanska, G., Aksan, N., \& Carlson, J. J. (2005). Temperament, relationships, and young children's receptive cooperation with their parents. Developmental Psychology, 41, 648.

Kochanska, G., DeVet, K., Goldman, M., Murray, K., \& Putnam, S. P. (1994). Maternal reports of conscience development and temperament in young children. Child Development, 65, $852-868$

Kochanska, G., \& Knaack, A. (2003). Effortful control as a personality characteristic of young children: Antecedents, correlates, and consequences. Journal of Personality, 71, 10871112. doi:10.1111/1467-6494.7106008

Kochanska, G., Murray, K., \& Coy, K. C. (1997). Inhibitory control as a contributor to 
conscience in childhood: From toddler to early school age. Child Development, 6, 263 277.

Kochanska, G., Murray, K., Jacques, T. Y., Koenig, A. L., \& Vandegeest, K. (1996). Inhibitory control in young children and its role in emerging internalization. Child Development, 67, 490-507.

Kotelnikova, Y., Mackrell, S. V. M., Jordan, P. L., \& Hayden, E. P. (2015). Longitudinal associations between reactive and regulatory temperament traits and depressive symptoms in middle childhood. Journal of Clinical Child and Adolescent Psychology, 44, 775-786. doi:10.1080/15374416.2014.893517

Kotelnikova, Y., Olino, T.M., Klein, D.N., Kryski, K.R., \& Hayden, E.P. (2016a). Higherand lower-order factor analyses of the Children's Behavior Questionnaire in early and middle childhood. Psychological Assessment, 28. 92-108. doi: 10.1037/pas0000153

Kotelnikova, Y., Olino, T.M., Klein, D.N., Mackrell, S.V.M., \& Hayden, E.P. (2016b). Higher- and lower-order factor analyses of the Temperament in Middle Childhood Questionnaire (TMCQ). Assessment. doi: 10.1177/1073191116639376

Kotelnikova, Y., Olino, T. M., Mackrell, S. V. M., Jordan, P. L., \& Hayden, E. P. (2013). Structure of observed temperament in middle childhood. Journal of Research in Personality, 47, 524-532. doi:10.1016/j.jrp.2013.04.013

Lamb, M. E., Chung, S. S., Wessels, H., Broberg, A. G., \& Hwang, C. P. (2002). Emergence and construct validation of the big five factors in early childhood: A longitudinal analysis of their ontogeny in Sweden. Child Development, 73, 1517-1524.

Laursen, B., Pulkkinen, L., \& Adams, R. (2002). The antecedents and correlates of agreeableness in adulthood. Developmental Psychology, 38, 591-603. 
Mackrell, S. V. M., Sheikh, H. I., Kotelnikova, Y., Kryski, K. R., Jordan, P. L., Singh, S. M., \& Hayden, E. P. (2014). Child temperament and parental depression predict cortisol reactivity to stress in middle childhood. Journal of Abnormal Psychology,123, 106-116. doi:10.1037/a0035612

Majdandžić, M., \& Van Den Boom, D.C . (2007). Multimethod longitudinal assessment of temperament in early childhood. Journal of Personality, 75, 121-167. doi:

10.1111/j.1467-6494.2006.00435.x

Mann, J. J., Brent, D. A., \& Arango, V. (2001). The neurobiology and genetics of suicide and attempted suicide: A focus on the serotonergic system.Neuropsychopharmacology, 24, 467-477. doi:10.1016/S0893-133X(00)00228-1

Markon, K. E., Krueger, R. F., \& Watson, D. (2005). Delineating the structure of normal and abnormal personality: An integrative hierarchical approach. Journal of Personality and Social Psychology, 88, 139-157. doi:10.1037/0022-3514.88.1.139

Marsh, H. W., Hau, K. T., \& Wen, Z. (2004). In search of golden rules: Comment on hypothesis testing approaches to setting cutoff values for fit indexes and dangers in overgeneralizing Hu \& Bentler's (1999) findings. Structural Equation Modeling, 11, 320341.

Martin, R. P., Wisenbaker, J., \& Huttunen, M. (1994). Review of factor analytic studies of temperament measures based on the Thomas-Chess structural model: Implications for the big five. The developing structure of temperament and personality from infancy to adulthood. (pp. 157-172) Lawrence Erlbaum Associates, Inc, Hillsdale, NJ.

McCrae, R. R., \& Costa, P. T. (1997). Personality trait structure as a human universal. American Psychologist, 52, 509-516. doi:10.1037/0003-066X.52.5.509 
McCrae, R. R., \& Terracciano, A. (2005). Personality profiles of cultures: Aggregate personality traits. Journal of Personality and Social Psychology, 89, 407-425. doi:10.1037/00223514.89.3.407

McCrae, R. R., Terracciano, A., De Fruyt, F., De Bolle, M., Gelfand, M. J., \& Costa, P. T., Jr. (2010). The validity and structure of culture-level personality scores: Data from ratings of young adolescents. Journal of Personality, 78, 815-838. doi:10.1111/j.14676494.2010.00634.x

Mervielde, I., Buyst, V., \& De Fruyt, F. (1995). The validity of the big-five as a model for teachers" ratings of individual differences among children aged 4-12 years. Personality and Individual Differences, 18, 525-534.

Morris, A. S., Silk, J.S., Steinberg, L., Terranova, A.M., \& Kithakye, M. (2010). Concurrent and longitudinal links between children's externalizing behavior in school and observed anger regulation in the mother-child dyad. Journal of Psychopathology and Behavioral Assessment, 32, 48-56, doi: 10.1007/s10862-009-9166-9

Muthén, L.K. and Muthén, B.O. (1998-2012). Mplus User's Guide. Seventh Edition. Los Angeles, CA: Muthén \& Muthén.

Nigg, J. T. (2006). Temperament and developmental psychopathology. Journal of Child Psychology and Psychiatry, 47, 395-422. doi:10.1111/j.1469-7610.2006.01612.x

Nigg, J. T., Goldsmith, H. H., \& Sachek, J. (2004). Temperament and attention deficit hyperactivity disorder: The development of a multiple pathway model. Journal of Clinical Child and Adolescent Psychology, 33, 42-53. doi: 10.1207/ S15374424JCCP3301_5

Olino, T. M., Durbin, C. E., Klein, D. N., Hayden, E. P., \& Dyson, M. W. (2013). Gender 
differences in young children's temperament traits: Comparisons across observational and parent-report methods. Journal of Personality, 81, 119-129.

Olino, T. M., Klein, D. N., Dyson, M. W., Rose, S. A., \& Durbin, C. E. (2010). Temperamental emotionality in preschool-aged children and depressive disorders in parents: Associations in a large community sample. Journal of Abnormal Psychology, 119, 468-478. doi:10.1037/a0020112

Pauli-Pott, U., Mertesacker, B., Bade, U., Haverkock, A., \& Beckmann, D. (2003). Parental perceptions and infant temperament development. Infant Behavior \& Development, 26, 27-48. doi:10.1016/S0163-6383(02)00167-4

Pesonen, A.-K., Raikkonen, K., Keskivaara, P., \& Keltikangas-Jarvinen, L. (2003). Difficult temperament in childhood and adulthood: Continuity from maternal perceptions to selfratings over 17 years. Personality and Individual Differences, 34, 19-31.

Presley, R., \& Martin, R. P. (1994). Toward a structure of preschool temperament: Factor structure of the temperament assessment battery for children. Journal of Personality, 62, $415-448$.

Putnam, S. P. (2012). Positive emotionality. New York, NY, US: Guilford Press, New York, NY. Rescorla, L. A., Bochicchio, L., Achenbach, T. M., Ivanova, M. Y., Almqvist, F., Begovac, I., . . . Verhulst, F. C. (2014). Parent-teacher agreement on children's problems in 21 societies. Journal of Clinical Child and Adolescent Psychology, 43, 627-642. doi:10.1080/15374416.2014.900719

Richard, H., Davis, D. W., \& Bums, B. M. (2008). An evaluation of the children's behavior questionnaire for use with children from low-income families. Journal of Early Childhood and Infant Psychology, 4, 111-123. 
Rothbart, M. K. (1981). Measurement of temperament in infancy. Child Development, 52, $569-578$.

Rothbart, M. K., Ahadi, S. A., Hershey, K. L., \& Fisher, P. (2001). Investigations of temperament at three to seven years: The children's behavior questionnaire. Child Development, 72, 1394-1408. doi:10.1111/1467-8624.00355

Rothbart, M.K. \& Bates, J.E. (2006). In Handbook of child psychology: Vol. 3 Social, emotional, and personality development (6th ed.), by Rothbart, Mary K., Bates, John E., 99-166. Hoboken, NJ, US: John Wiley \& Sons Inc.

Shankman, S. A., Tenke, C. E., Bruder, G. E., Durbin, C. E., Hayden, E. P., \& Klein, D. N. (2005). Low positive emotionality in young children: Association with EEG asymmetry. Development and Psychopathology, 17, 85-98. doi:10.1017/ S0954579405050054

Shiner, R. L., \& DeYoung, C. G. (2013). The structure of temperament and personality traits: A developmental perspective. The oxford handbook of developmental psychology, vol. 2: Self and other. (pp. 113-141) Oxford University Press, New York, NY.

Shiner, R. L., Masten, A. S., \& Tellegen, A. (2002). A developmental perspective on personality in emerging adulthood: Childhood antecedents and concurrent adaptation. Journal of Personality and Social Psychology, 83, 1165-1177.

Simonds, J. \& Rothbart, M. K. (2004). The Temperament in Middle Childhood Questionnaire (TMCQ): A computerized self-report measure of temperament for ages 7 10. Poster session presented at the Occasional Temperament Conference, Athens, GA. Spinrad, T. L., Eisenberg, N., Gaertner, B., Popp, T., Smith, C. L., Kupfer, A., .. Hofer, C. (2007). Relations of maternal socialization and toddlers effortful control to childrens 
adjustment and social competence. Developmental Psychology, 43, 1170-1186. doi:10.1037/0012-1649.43.5.1170

Tackett, J. L. (2006). Evaluating models of the personality-psychopathology relationship in children and adolescents. Clinical psychology review, 26, 584-599.

Tackett, J. L., Martel, M. M., \& Kushner, S. C. (2012a). Temperament, externalizing disorders, and attention-deficit/hyperactivity disorder. New York, NY, US: Guilford Press, New York, NY.

Tackett, J. L., Slobodskaya, H. R., Mar, R. A., Deal, J., Halverson, C. F., Baker, S. R., . . . Besevegis, E. (2012b). The hierarchical structure of childhood personality in five countries: Continuity from early childhood to early adolescence. Journal of Personality, 80, 847-879. doi:10.1111/j.1467-6494.2011.00748.x

Tellegen, A. (1985). Structure of mood and personality and their relevance to assessing anxiety, with an emphasis on self-report. In A. H. Tuma \& J. D. Maser (Eds.), Anxiety and the anxiety disorders (pp. 681-706). Hillsdale, NJ: Erlbaum.

Thomas, A., \& Chess, S. (1977). Temperament and development. Brunner/Mazel, Oxford.

Watson, D., \& Clark, L. A. (1993). In Wegner D. M., Pennebaker J. W. (Eds.), Behavioral disinhibition versus constraint: A dispositional perspective. Englewood Cliffs, NJ, US: Prentice-Hall, Inc, Englewood Cliffs, NJ.

Wu, T., Dixon, W. E., Jr., Dalton, William T., I.,II, Tudiver, F., \& Liu, X. (2011). Joint effects of child temperament and maternal sensitivity on the development of childhood obesity. Maternal and Child Health Journal, 15, 469-477. doi: 10.1007/s10995-0100601-z

Zalsman, G., Patya, M., Frisch, A., Ofek, H., Schapir, L., Blum, I., . . Tyano, S. (2011). Association of polymorphisms of the serotonergic pathways with clinical traits of 
impulsive-aggression and suicidality in adolescents: A multi-center study. The World Journal of Biological Psychiatry, 12, 33-41. doi:10.3109/15622975.2010.518628

Zhang, H., Ishigaki, T., Tani, K., Chen, K., Shih, J. C., Miyasato, K., . . Ohara, K. (1997). Serotonin 2A receptor gene polymorphism in mood disorders. Biological Psychiatry, 41, 768-773.

Zentner, M., \& Bates, J. E. (2008). Child temperament: An integrative review of concepts, research programs, and measures. European Journal of Developmental Science, 2, 7-37. 
Table 1.

Bivariate Associations Between Major Study Variables

\begin{tabular}{|c|c|c|c|c|c|c|c|c|c|c|c|c|c|c|c|}
\hline & PA & AntPA & Int & Activ & Compl & Sociab & Imp & Fear & Ang & $\mathrm{Sad}$ & $\mathrm{BI}$ & LowEC & Sex & PPVT & Income \\
\hline PA & -- & $.67 * *$ & $.57 * *$ & $.55 * *$ & -.01 & $.46 * *$ & $.32 * *$ & .04 & -.03 & -.04 & $-.12 *$ & $.18 * *$ & .00 & $.12 *$ & .10 \\
\hline Ant PA & & -- & $.57 * *$ & $.44 * *$ & .04 & $.50 * *$ & $.27 * *$ & -.03 & .01 & -.09 & $-.16 *$ & $.18^{* *}$ & -.09 & $.15^{* *}$ & .04 \\
\hline Int & & & -- & $.52 * *$ & $.11 *$ & $.67 * *$ & $.35^{* *}$ & -.10 & .05 & $-.13 * *$ & $-.34 * *$ & $.29 * *$ & $-.19 * *$ & $.18 * *$ & .06 \\
\hline Activ & & & & -- & $-.44 * *$ & $.52 * *$ & $.78 * *$ & -.08 & $.33 * *$ & .01 & $-.30 * *$ & $.39 * *$ & $-.26 * *$ & .01 & .01 \\
\hline Compl & & & & & -- & -.09 & $-.64 * *$ & -.01 & $-.47 * *$ & $-.31 * *$ & .01 & $-.39 * *$ & $.19 * *$ & $.29 * *$ & .07 \\
\hline Sociab & & & & & & -- & $.42 * *$ & -.03 & $.14 * *$ & .09 & $-.24 * *$ & $.41 * *$ & $-.16^{* *}$ & $.18 * *$ & $.12 *$ \\
\hline Imp & & & & & & & -- & -.07 & $.53 * *$ & $.15^{* *}$ & $-.28 * *$ & $.50 * *$ & $-.34 * *$ & -.09 & -.02 \\
\hline Fear & & & & & & & & -- & .01 & .04 & $.32 * *$ & .04 & .09 & -.01 & -.02 \\
\hline Ang & & & & & & & & & -- & $.35 * *$ & .01 & $.27 * *$ & $-.19 * *$ & $-.13 * *$ & .02 \\
\hline Sad & & & & & & & & & & -- & $.21 * *$ & $.13 * *$ & .01 & -.04 & .08 \\
\hline BI & & & & & & & & & & & -- & -.09 & $.14 * *$ & -.04 & .05 \\
\hline LowEC & & & & & & & & & & & & -- & $-.23 * *$ & $-.15 * *$ & -.01 \\
\hline Sex & & & & & & & & & & & & & -- & .07 & -.02 \\
\hline PPVT & & & & & & & & & & & & & & -- & $.11 *$ \\
\hline$M$ & 0 & 1.32 & 1.90 & 1.67 & 2.01 & 1.70 & .79 & .40 & .46 & -.18 & .01 & .45 & -- & 112 & -- \\
\hline$S D$ & .74 & .35 & .32 & .35 & .37 & .44 & .33 & .23 & .33 & .26 & 13.64 & .27 & -- & 14.06 & -- \\
\hline
\end{tabular}

Note. ${ }^{* *} p \leq .01, * p \leq .05, \mathrm{PA}=$ positive affect, AntPA $=$ anticipatory positive affect, Int $=$ interest, Activ $=$ activity, Compl $=$ compliance, Imp = impulsivity, Ang = anger, Sad = sadness, BI = behavioral inhibition, LowEC = low levels of effortful control, Income = family income; child sex was coded as $1=$ boy $2=$ girl; income was coded as $1<\$ 20,000 ; 2=\$ 20,000-\$ 40,000 ; 3=$ $\$ 40,001-\$ 70,000 ; 4=\$ 70,001-\$ 100,000 ; 5>\$ 100,001$. 
Table 2.

Structure of Temperament in Preschoolers: ESEM Results

\begin{tabular}{|c|c|c|c|c|c|c|c|c|}
\hline \multirow[b]{2}{*}{ Variable Name } & \multicolumn{2}{|c|}{ F1=PA/Activity } & \multicolumn{2}{|c|}{ F2 = Impulsivity/Anger } & \multicolumn{2}{|c|}{ F3 = Dysphoria } & \multicolumn{2}{|c|}{ F4 = Surgency } \\
\hline & $\begin{array}{l}\text { Standard } \\
\text { Estimate }\end{array}$ & $p$ value & $\begin{array}{l}\text { Standard } \\
\text { Estimate }\end{array}$ & $p$ value & $\begin{array}{l}\text { Standard } \\
\text { Estimate }\end{array}$ & $p$ value & $\begin{array}{l}\text { Standardi } \\
\text { Estimate }\end{array}$ & $p$ value \\
\hline PA & $\underline{0.99}$ & 0.00 & 0.02 & 0.24 & 0.18 & 0.03 & -0.02 & 0.21 \\
\hline Anticipatory PA & $\overline{0.61}$ & 0.00 & -0.03 & 0.38 & 0.00 & 0.91 & 0.22 & 0.00 \\
\hline Interest & $\overline{\mathbf{0 . 3 2}}$ & 0.00 & -0.01 & 0.82 & -0.21 & 0.00 & 0.53 & 0.00 \\
\hline Activity & $\overline{0.46}$ & 0.00 & $\underline{0.63}$ & 0.00 & -0.09 & 0.06 & 0.07 & 0.12 \\
\hline Compliance & $\overline{0.00}$ & 0.96 & -0.79 & 0.00 & -0.21 & 0.03 & 0.14 & 0.11 \\
\hline Sociability & 0.05 & 0.34 & $\overline{0.02}$ & 0.67 & 0.02 & 0.68 & $\underline{0.88}$ & 0.00 \\
\hline Impulsivity & 0.22 & 0.00 & $\underline{0.91}$ & 0.00 & -0.05 & 0.42 & $\overline{0.00}$ & 0.98 \\
\hline Fear & 0.10 & 0.15 & $\overline{-0.07}$ & 0.50 & $\underline{0.47}$ & 0.00 & 0.03 & 0.60 \\
\hline Anger & -0.09 & 0.13 & $\underline{0.61}$ & 0.00 & $\overline{0.12}$ & 0.14 & 0.00 & 0.95 \\
\hline Sadness & -0.09 & 0.15 & $\overline{0.19}$ & 0.26 & $\underline{0.53}$ & 0.00 & 0.16 & 0.26 \\
\hline BI & 0.02 & 0.24 & -0.17 & 0.26 & 0.58 & 0.00 & -0.11 & 0.38 \\
\hline LowEC & -0.02 & 0.67 & $\underline{0.40}$ & 0.00 & $\overline{0.10}$ & 0.09 & $\underline{0.33}$ & 0.00 \\
\hline
\end{tabular}

Note. $\mathrm{PA}=$ positive affect; Anticipatory $\mathrm{PA}=$ anticipatory positive affect; $\mathrm{BI}=$ behavioral inhibition; LowEC $=$ low levels of effortful control; standardized loadings $\geq .30$ are highlighted and underlined. 
Table 3.

Bivariate Associations Between ESEM Factors

\begin{tabular}{lcccc}
\hline & $\mathrm{F} 1=\mathrm{PA} /$ Activity & $\mathrm{F} 2=$ Impulsivity/Anger & $\mathrm{F} 3=$ Dysphoria & $\mathrm{F} 4=$ Surgency \\
$\mathrm{F} 1=$ PA/Activity & -- & .11 & $-.30^{* *}$ & $.52^{* *}$ \\
$\mathrm{~F} 2=$ Impulsivity/Anger & & - & -.02 & $.34^{* *}$ \\
$\mathrm{~F} 3=$ Dysphoria & & & -- & $-.18^{*}$ \\
$\mathrm{~F} 4=$ Surgency & & & -- \\
\hline
\end{tabular}

Note. ${ }^{* *} p \leq .01,{ }^{*} p \leq .05 ; \mathrm{PA}=$ positive affect. 


\section{Table 4.}

Bivariate Associations Between Temperament Factors and Concurrent Parent-Reported Symptoms of Psychopathology

\begin{tabular}{l|l|l|l}
\hline \multirow{2}{*}{ Temperament Factor } & \multicolumn{3}{|c}{ CBCL Scales } \\
\cline { 2 - 4 } & Withdrawn/Depressed & Attentional Problems & Aggressive Behavior \\
\hline PA/Activity & $-.21^{* *}$ & $.10 \dagger$ & .01 \\
Impulsivity/Anger & $-.20^{* *}$ & $.24^{* *}$ & $.17^{* *}$ \\
Surgency & $-.25^{* *}$ & $.10 \dagger$ & -.05 \\
Dysphoria & $.15^{* *}$ & -.06 & .01 \\
\hline$M$ & 1.23 & 2.20 & 5.75 \\
$S D$ & 1.32 & 1.96 & 4.63 \\
\hline
\end{tabular}

Note. ${ }^{* *} p \leq .01, * p \leq .05, \dagger .05<\mathrm{p}<.10$; PA = positive affect; temperament factors were calculated as sums of z-score transformed variables from the ESEM model (Table 2). 
.521

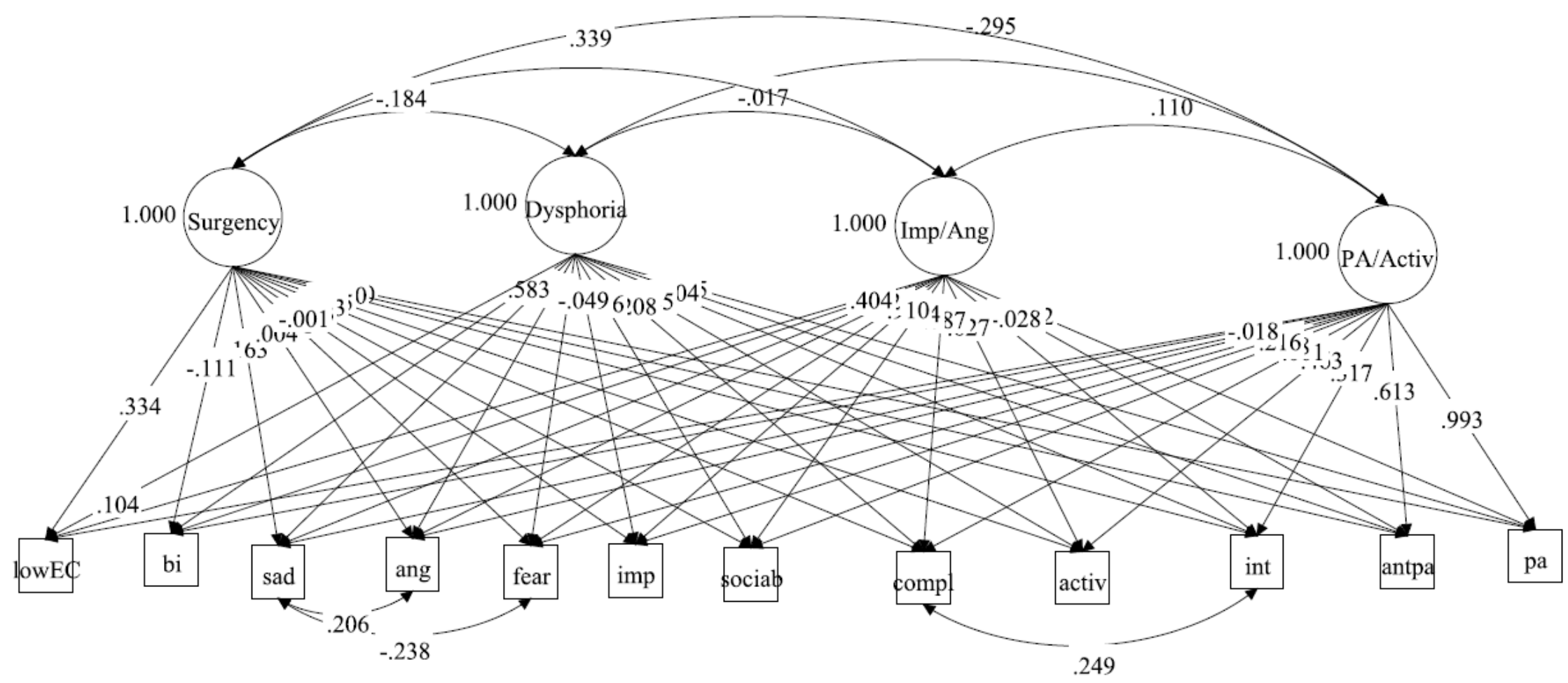

Figure 1. Structure of Temperament in Preschoolers; factors: Imp/Ang = Impulsivity/Anger, PA/Activ = Positive Affect/Activity; observed indicators: lowEC $=$ low levels of effortful control, BI $=$ behavioral inhibition, sad $=$ sadness, ang $=$ anger, imp $=$ impulsivity, sociab $=$ sociability, $\mathrm{compl}=$ compliance, activ $=$ activity, int $=$ interest, antpa $=$ anticipatory positive affect, and pa $=$ positive affect. 


\section{Supplementary Material}

\section{Description of NY Sample}

The NY sample of 559 preschoolers (302 boys, $\left.54 \% ; M_{\text {age }}=3.52 ; S D_{\text {age }}=.26\right)$ and their primary caregivers $\left(96 \%\right.$ mothers; $\left.M_{a g e}=35.98 ; S D_{a g e}=4.35\right)$ was recruited through commercial mailing lists. Children with significant medical conditions or developmental disabilities were excluded. Children were mostly Caucasian (87\%) and of average cognitive ability $(M=103, S D$ = 14; PPVT; Dunn \& Dunn, 2007). The NY sample consisted of mainly middle class families $(1 \%<\$ 20,000 ; 4 \%=\$ 20,000-\$ 40,000 ; 21 \%=\$ 40,001-\$ 70,000 ; 35 \%=\$ 70,001-\$ 100,000 ;$ $39 \%>\$ 100,001)$.

\section{Assessment of Temperament in NY Sample}

Lab-TAB (Goldsmith et al., 1995) episodes and procedures were identical to those used in the London, ON sample with one exception: the two tasks described below were administered only in the NY sample instead of the Puzzle with Parent and Jumping Spider tasks in the London, ON Lab-TAB.

Exploring New Objects. The child was left alone to explore a set of novel and ambiguous stimuli, such as pretend mice in a cage, sticky water-filled gel balls, a mechanical bird, a mechanical spider, and a pretend skull covered under a blanket. After five minutes, the experimenter returned and asked the child to play with each object.

Arc of Toys. The child was allowed to play freely by him/herself in a room with toys for a few minutes, following which the experimenter returned and asked the child to clean up the toys.

\section{Video Coding Procedures in NY Sample}

Video coding procedures used in the NY sample were similar to those described for the 
sample from London, ON. However, there were some differences in the composition of the BI variable. In the NY sample, the BI variable consisted of an average of standardized BI ratings from the Risk Room, Stranger Approach, and Exploring New Objects episodes (in the sample from London, ON the Jumping Spider task was administered instead of Exploring New Objects task). Internal consistency statistics and mean ICCs for the NY sample ranging between $.50-.87$ and $.40-.98$ respectively were previously described by Dyson et al. (2012), and will not be described here.

\section{Confirmatory Factor Analysis in NY Sample}

The ESEM four-factor solution presented in Table 2 of the main manuscript was crossvalidated in the NY sample using a confirmatory factor analysis (CFA). Despite some concerns that the use of CFA can lead to misspecifications or distortions of the structural relations among different items and factors in temperament/personality data (Church \& Burke, 1994; Hopwood \& Donnellan, 2010; Marsh et al., 2009), we felt that this approach was appropriate for replication analyses given our specific hypotheses regarding the expected structure of observed child temperament. Misspecification concerns were addressed by retaining most of the cross-loadings present in the original model and examination of model estimates and modification indices (MIs) during post-hoc model fitting to identify additional adjustments to improve model fit. Conventional CFA goodness-of-fit criteria were used, with values above .90 and .95 for the CFI used as indices of acceptable and excellent fit (Hu \& Bentler, 1999). Similarly, RMSEA values that were lower than .05 were treated as indicating a close fit, with values up to 0.08 indicating acceptable fit (Marsh, Hau, \& Wen, 2004) of the CFA models. The variances of the latent factors were fixed to 1.0 in order to obtain significance values for all of the paths.

To construct a CFA model for replication in the NY sample, observed indicators with 
loadings $\geq .40$ (see Table 2 ) were considered; one cross-loading was eliminated by selecting a factor with the highest (primary) loading (i.e., interest loaded on Surgency only). PA, anticipatory PA, and activity loaded on the PA/Activity factor; impulsivity, anger, low EC, compliance, and activity loaded on the Impulsivity/Anger factor. Although the ESEM solution showed that activity had a primary loading on the Impulsivity/Anger factor, it was retained in the PA/Activity factor to increase its stability. Fear, sadness, and BI loaded on the Dysphoria factor and interest, sociability, and low EC loaded on the Surgency factor. Low EC was retained in the Surgency factor to increase its stability.

The model fit of this initial four-factor solution was poor (RMSEA =.17; CFI =.76); thus, model estimates and modification indices (MIs) were examined and post-hoc model fitting was conducted. A covariance path between NA and PA/Activity factors was not significant and was subsequently constrained to zero. After examining MIs, Low EC was removed from the Surgency factor and replaced with a related construct of compliance (see final model in Figure A). Similarly based on MIs, sociability and BI were made to load on a factor with PA variables, which was re-labelled as PA/Sociability, and activity was set to load on a factor re-labelled as Agreeableness/Engagement (see Figure A). Additionally, anger was allowed to cross-load on Dysphoria, which was subsequently re-labelled as NA, and Impulsivity/Anger factors. Finally, several covariance paths were added between residuals of observed indicators based on examination of MIs (see Figure A). The final CFA model in the replication sample is presented in Figure A below; it has an acceptable fit $(\mathrm{RMSEA}=.07$; CFI $=.97)$. While Impulsivity/Anger and NA factors remained mostly unmodified, the other two factors exchanged sociability and activity indicators, suggesting that the model derived in sample from London, ON could only be replicated with significant revisions. 


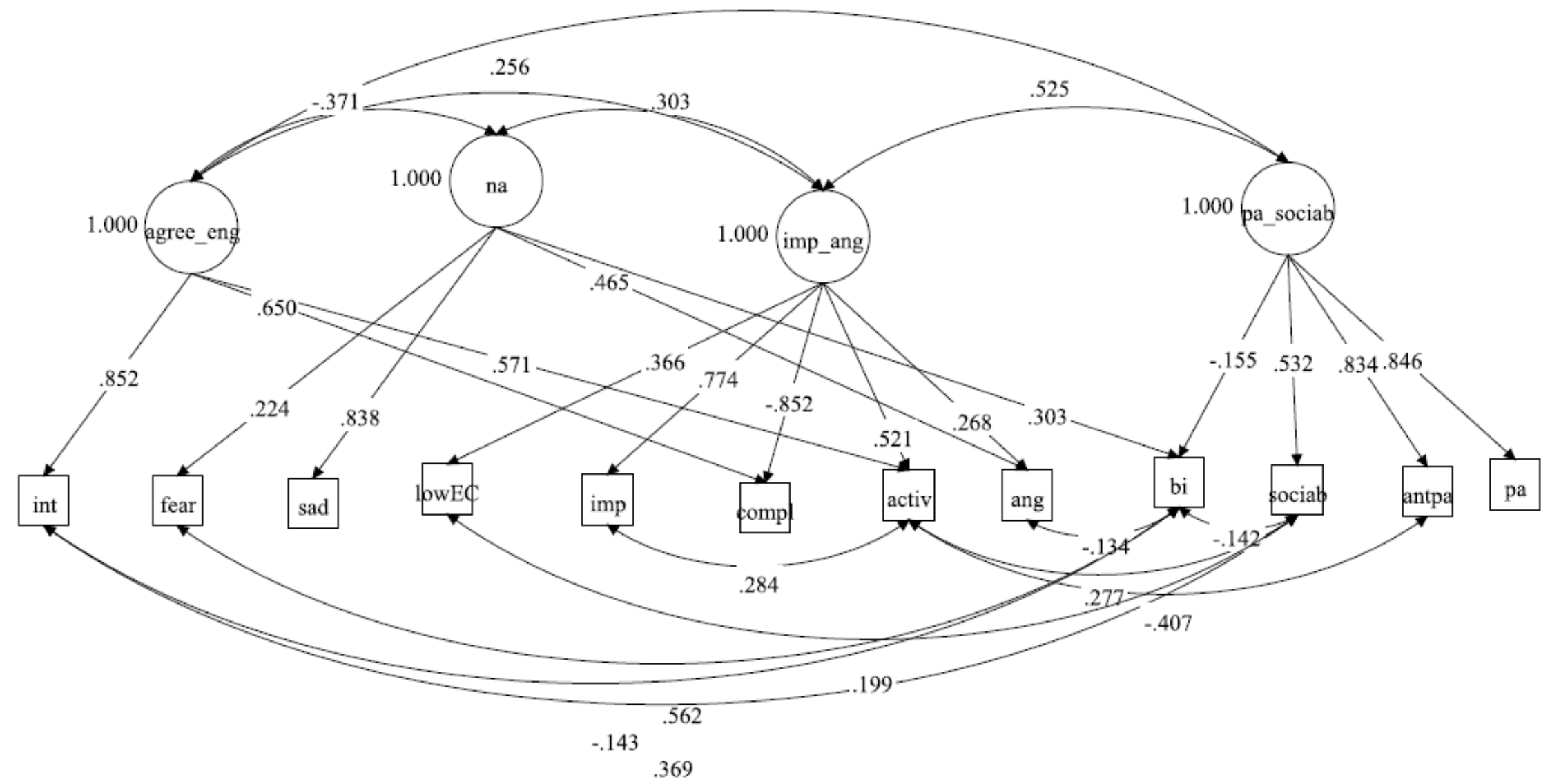

Figure A. CFA in NY Sample; factors: agree_eng = Agreeableness/Engagement, na = Negative Affect, imp_ang = Impulsivity/Anger, pa_sociab = Positive Affect/Sociability; observed indicators: lowEC = low levels of effortful control, BI = behavioral inhibition, sad = sadness, ang = anger, imp = impulsivity, sociab = sociability, compl = compliance, activ=activity, int $=$ interest, antpa $=$ anticipatory positive affect, and $\mathrm{pa}=$ positive affect. 


\section{General Discussion}

Historically, the term personality has been used to describe a broader and more finegrained range of individual differences than temperament; nevertheless, both capture relatively stable patterns of behavior across situations (Pytlik Zillig, Hemenover, \& Dienstbier, 2002; Wilt \& Revelle, 2009) that emerge from the interplay between genetic factors and environmental influences (Clark \& Watson, 2008; Shiner \& DeYoung, 2013). A unified taxonomy of the primary dimensions captured by models of temperament and personality is essential for studying covariation among traits, their developmental trajectories across the lifespan, and associations with developmental outcomes (Shiner \& DeYoung, 2013). While there is a substantial literature focused on the structure of adult personality, relevant work on child temperament/personality has lagged behind. The three studies that comprise this dissertation attempt to address this gap by using different measurement approaches to address the nature of the structure of temperament in early and middle childhood.

In the first two studies, we examined the higher- and lower-order structure of motherreported child temperament based on two widely used questionnaire measures, the Children's Behavior Questionnaire (CBQ; Rothbart, Ahadi, Hershey, \& Fisher, 2001) and the Temperament in Middle Childhood Questionnaire (TMCQ; Simonds \& Rothbart, 2004). Although previous work has investigated the higher-order structure of the CBQ using factor analyses of the lowerorder scales (e.g., Rothbart et al., 2001), no previous work has examined the structure of the $\mathrm{CBQ}$ at the item level. More specifically, the CBQ was developed via a purely top-down, theorydriven approach, with no empirical validation of the lower-order scales. Similarly, the parentreport version of the TMCQ has not been examined at the higher- or lower-order levels, although meagre structural work has been done on the child self-report version of the TMCQ (Simonds \& 
Rothart, 2004). The first two studies extended our knowledge of the structure of these measures via both lower- and higher-order factor analyses. In the third study, we extended the small literature on the structure of observed child temperament. Results from the three studies with respect to the higher-order structure of child temperament are summarized in Table 1 (lowerorder findings derived from analyses of the two questionnaire measures are addressed later).

\begin{tabular}{|c|c|c|c|}
\hline $\begin{array}{c}\text { CBQ } 3 \text { y.o. } \\
\text { Maternal report } \\
\end{array}$ & $\begin{array}{c}\text { CBQ 5/6 y.o. } \\
\text { Maternal report } \\
\end{array}$ & $\begin{array}{c}\text { TMCQ } 9 \text { y.o. } \\
\text { Maternal report } \\
\end{array}$ & $\begin{array}{c}\text { Observed } 3 \text { y.o. } \\
\text { Lab-TAB } \\
\text { (Goldsmith et al., 1995) } \\
\end{array}$ \\
\hline F1: Sensation Seeking & F1: Sensation Seeking & & \\
\hline $\begin{array}{l}\text { F2: Low EC/Disinhibition } \\
+ \text { Anger/Sadness }\end{array}$ & F2: Disinhibition/Anger & $\begin{array}{l}\text { F1: Impulsivity } \\
+ \text { Anger/Sadness }\end{array}$ & F1: Impulsivity/ Anger \\
\hline $\begin{array}{l}\text { F3: Low NA + } \\
\text { Soothability }\end{array}$ & F3: Soothability & F2: NA & $\begin{array}{l}\text { F2: Dysphoria/ Fear \& } \\
\text { Sadness }\end{array}$ \\
\hline $\begin{array}{l}\text { F4: Smiling/Laughter + } \\
\text { Approach/Positive } \\
\text { Anticipation }\end{array}$ & $\begin{array}{l}\text { F4: Smiling/Laughter }+ \\
\text { Approach/Positive } \\
\text { Anticipation }\end{array}$ & $\begin{array}{l}\text { F3: Social } \\
\text { Dominance/ } \\
\text { Perceptive }\end{array}$ & $\begin{array}{l}\text { F3: PA/Activity; } \\
\text { F4: Surgency }\end{array}$ \\
\hline
\end{tabular}

Table 1. Higher-order structure of child temperament based on child age and method of assessment from Kotelnikova, Kryski, Olino, Klein, \& Hayden (2016), Kotelnikova, Olino, Klein, Kryski, \& Hayden (2016), and Kotelnikova, Olino, Klein, Mackrell, \& Hayden (2016).

Despite differences in methodology and age, an overview of the table suggests some consensus regarding the higher-order structure of child temperament, as well as highlighting dimensions of child temperament that bear close resemblance to those found in prominent models of adult temperament/personality. First, we found an Extraversion/Surgency-like, Neuroticism/Negative Affect (NA)-like, and Disinhibition/Low Effortful Control (EC)-like factor in all three studies and across the two methods, consistent with prominent extant three-to five-factor models of child and adult temperament/personality (e.g., McCrae \& Costa, 1997; Rothbart et al., 2001; Tellegen, 1985; Watson \& Clark, 1993). The Extraversion/Surgency-like factors of the CBQ at ages 3 and 5/6 and the observed temperament model contained facets tapping positive affect (PA). Since the Smiling/Laughter scale was omitted from the TMCQ by 
its developers (Simonds, 2006; Simonds \& Rothbart, 2004), its Extraversion/Surgency-like higher-order factor (i.e., Social Dominance/Perceptive) could therefore not contain an affective component. All four higher-order structures presented in Table 1 contain a factor tapping impulsivity/anger and low EC, which approximates several regulatory factors found in extant models of child and adult temperament/personality (e.g., McCrae \& Costa, 1997; Rothbart et al., 2001; Shiner \& DeYoung, 2013; Tellegen, 1985; Watson \& Clark, 1993). Finally, all the higherorder models in Table 1 contain an NA-like factor; however, its composition varied across methods. More specifically, it consisted of high soothability and low fear in the higher-order structure of the CBQ at both ages, fear, sadness, and shyness in the TCMQ, and fear, behavioral inhibition, and sadness in the observed temperament model.

Despite these commonalities, our analyses also yielded some unexpected results. While activity level is thought to be another higher-order construct essential to capturing the nature of child temperament (Shiner \& DeYoung, 2013), we did not find such a factor in any of our models. Instead, activity level loaded on the Disinhibition/Anger factor in the first study (Kotelnikova et al., 2016b), and cross-loaded on the Impulsivity and Social Dominance/Perceptive factors and the Impulsivity/Anger and PA/Activity factors in the second and third studies, respectively (Kotelnikova et al., 2016a; 2016c). While contrary to theory, these results are consistent with previous findings that activity is associated with both impulsivity/dysregulation and positive engagement in children (e.g., De Pauw, Mervielde, \& Van Leeuwen 2009; Goldberg, 2001; John, Naumann, \& Soto, 2008; Lamb, Chuang, Wessels, Broberg, \& Hwang, 2002), indicating that this trait may emerge as a part of a higher-order Disinhibition or Extraversion/PA factor.

Another somewhat unexpected finding was that anger clustered with EC traits across all 
three studies, as opposed to loading with other NA facets (i.e., fear and sadness). Although consistent with related work on children's emotional development (De Pauw et al., 2009; Digman \& Shmelyov, 1996), this finding is perhaps surprising as most prominent models of adult temperament/personality posit a single NA/Neuroticism factor that includes fear, anger, and sadness (e.g., McCrae \& Costa, 1997; Tellegen, 1985; Watson \& Clark, 1993). This finding may be at least partially attributed to the fact that numerous items that were supposed to tap these constructs of the CBQ and the TMCQ functioned poorly at the lower-order level, and were therefore excluded from higher-order analyses (Kotelnikova et al., 2016b; 2016c). However, our current analyses of laboratory data did not recover an NA factor consisting of fear, anger, and sadness in preschoolers, and neither did Dyson, Olino, Durbin, Goldsmith, and Klein (2012) in their study of observed temperament in preschoolers. Dyson et al. (2012) posited that, as children get older, anger, sadness, and fear would become increasingly intercorrelated, coalescing into adult Neuroticism; however, at which point in development this process might occur remains unclear. Shiner and DeYoung (2013) have also discussed an alternative possibility of increased differentiation of child temperament traits over time, and some existing studies provide evidence for such a developmental trajectory. For example, in a study of observed temperament in 7-yearolds, Kotelnikova, Olino, Mackrell, Jordan, and Hayden (2013) found differentiation of NA into three separate factors, consistent with a developmental literature showing increased differentiation of this construct in childhood (Camras, Oster, Campos, Campos, Ujiie, Miyake et al., 1998; Izard, Fantauzzo, Castle, Haynes, Rayias, \& Putnam, 1995). The results of a recent behavior genetic study (Clifford, Lemery-Chalfant, \& Goldsmith, 2015) also indicate that observed fear, anger, and sadness are genetically distinct from each other. To our knowledge, no study has investigated the underlying structure of observational measures of adult temperament; 
thus, it is possible that observational studies might reveal continued differentiation among facets of NA in adulthood, such that evidence for strong covariance between sadness, anger, and fear is only apparent when self-report methods are used.

Despite the similarities we found across models, the three studies also indicate that the structure of child temperament differs depending on whether parent-report or observational measures are used (although we did not test this specifically). This is unsurprising in light of work showing low agreement between parent-reported and observed temperament (e.g., Durbin, Hayden, Klein, \& Olino, 2007; Durbin \& Wilson, 2012; Hayden, Durbin, Klein, \& Olino, 2010). Overall, parent reports and observational methods appear to provide unique information about child temperament, making it important to understand when the cost and time-commitment associated with observational methods are justified. In order to answer this question, further work comparing the convergent and discriminant validity of parent-report measures to observational methods is needed. Examining the criterion and predictive validity of these methods is equally important (e.g., Hayden, Klein, \& Durbin, 2005; Rohlf \& Krahé, 2015). However, further work improving the content of the CBQ and TMCQ is likely necessary before such comparisons can be made, given that our results suggest that core aspects of temperament thought crucial to developmental outcomes are not well-tapped by these measures. For example, many items from the CBQ and TMCQ intended to capture aspects of NA (i.e., Anger/Frustration, Sadness, Fear, Discomfort, and Soothability/Falling Reactivity) and EC (i.e., Activation Control, Inhibitory Control, Low Intensity Pleasure, and Perceptual Sensitivity) were excluded due to poor functioning. Given the key role these constructs are thought to play in shaping adaptive and maladaptive development (e.g., Caspi, 2000; Kochanska \& Knaack, 2003; Kotelnikova, Mackrell, Jordan, \& Hayden, 2015; Nigg, 2006), improved items tapping such content will need 
to be added to these measures. In particular, NA and EC items that consistently functioned poorly across ages and questionnaires should likely be removed from the CBQ and TMCQ. New versions of these measures comprised of new and extant NA and EC items that adequately loaded on relevant scales could next be validated in large samples of children using item- and factor-level exploratory factor analyses. Structural analyses could be followed up by item response theory (IRT) analyses for a more detailed examination of item functioning and further refining of the measure, with the added goal of reducing these lengthy measures.

In light of their conceptual and content overlap (i.e., a third of the TMCQ items were taken verbatim from the CBQ), we anticipated some degree of consistency of the higher-order structures between the two measures. However, this was not the case: only one of the four factors derived for the higher-order structure of the CBQ in 3- and 5/6-year-olds (Disinhibition/Anger) showed conceptual overlap with a TMCQ factor (Impulsivity/Anger). It is possible that these structural differences resulted from differences in the original content of the TMCQ and CBQ (e.g., the Smiling/Laughter scale was not included in the TMCQ; Affiliation and Fantasy/Openness scales were added to the TMCQ). Similarly, developmental changes in child temperament structure over time also could have contributed to the differences. Shiner and DeYoung (2013) proposed that increasing differentiation of traits is found as children age. As a result, the relatively narrow range of temperament traits in younger children transforms into a more complex structure of traits during middle childhood (Shiner \& DeYoung, 2013). To tap this developmental trajectory, Simonds and Rothbart (2004) proposed an additional fourth factor, Sociability/Affiliation, resembling Agreeableness from the Five Factor Model of adult personality (FFM; McCrae \& Costa, 1997). However, the Sociability/Affiliation factor was not recovered as a higher-order construct in our analyses (see Table 1), suggesting that different 
content may need to be added to tap emerging Agreeableness in middle childhood.

We also addressed the extent of similarity between the higher-order structures developed using our "bottom-up" approach to the CBQ and the TMCQ to those proposed by Rothbart and colleagues based on "top-down” models (i.e., Rothbart et al., 2001; Simonds \& Rohbart, 2004). Any similarities were marginal at best. Rothbart et al. (2001) found a Surgency factor based on higher-order EFA analyses of the CBQ scales. Such a factor did not emerge in our analyses, although two dimensions tapping related behaviors did, with one comprised of Smiling/Laughter and Approach/Positive Anticipation items, and the other capturing behaviors related to sensationseeking (Kotelnikova et al., 2016b). Also in contrast to Rothbart's three-factor model of temperament in preschoolers, we did not recover distinct NA and EC factors at either age 3 or 5/6 (see Table 1). Similar findings were obtained in our higher-order EFA of the TMCQ; in contrast to Simonds and Rothbart (2004), the third factor of our TMCQ model (Social Dominance/Perceptive) was a mixture of constructs representing lower-order scales tapping Extraversion/Surgency, Fantasy/Openness, and Perceptual Sensitivity (Kotelnikova et al., 2016c). Overall, our results indicate that bottom-up analyses did not replicate the higher- or the lower-order structures of the CBQ and the TMCQ derived via a purely top-down theory-based approach (i.e., Rothbart et al., 2001; Simonds \& Rothbart, 2004).

The structure of observed temperament in preschoolers derived in the third study also showed mixed resemblance to the structure hypothesized for temperament in preschoolers based on parent-report measures (i.e., Rothbart et al., 2001). Some similarities are seen in the PA/Extraversion-like and EC-like factors present in both our derived structure of observed temperament and that proposed by Rothbart and colleagues (2001) for this age group (see Table 1). In particular, Rothbart's Extraversion/Surgency factor includes scales tapping PA and activity 
level (Rothbart et al., 2001), consistent with the composition of the PA/Activity factor in our observed temperament structure. Similarly, there is overlap between Rothbart's EC factor (Rothbart et al., 2001) and observed Impulsivity/Anger factor in our third study (i.e., both include a lower-order facet tapping inhibitory control). However, there are also significant differences in the composition of the observed Impulsivity/Anger and Dysphoria factors derived in our third study (Kotelnikova et al., 2016a) and their counterparts in Rothbart and colleagues' model (i.e., EC and NA).

Indeed, the four-factor model derived from our observation measures shows stronger similarity to the only other previous study of observed temperament in preschoolers by Dyson and colleagues (2012). In particular, three of the four factors of our model (i.e., PA/Activity, Impulsivity/Anger, and Surgency) were highly similar to those reported by Dyson et al. (2012), albeit in a five-factor model (i.e., PA/Interest, Constraint vs. Impulsivity, and Sociability). Similarly, neither of us were able to recover a single NA factor consisting of observed fear, anger, and sadness in preschoolers.

\section{Strengths and Weaknesses of the Three Studies and Future Directions}

All three studies that comprise this thesis have important strengths. Older models of child temperament have relied almost exclusively on relatively small samples, and have failed to conduct rigorous tests of structure, relying solely on top-down, theory-driven approaches. Our first two studies (range of $N s=654$ - 944) used exceptionally large samples for this literature, which allowed us to conduct factor analyses at the item level, examining the lower-order structure of two widely used measures of temperament in early and middle childhood. To our knowledge, these are the first such studies of their kind.

Our third study makes a contribution toward understanding the nature of child 
temperament as assessed by observational methods. Although caregiver reports of child temperament are affordable, easy to use, efficient, and rely on parents' extensive knowledge of their children's behavior across multiple contexts (Pauli-Pott, Mertesacker, Bade, Haverkock, \& Beckmann, 2003), concerns have been raised regarding their validity (e.g., Kagan, 1998), and recent work suggests that such methods are contaminated by parent characteristics, including caregiver personality, mood state, availability biases, and symptoms of psychopathology (Durbin et al., 2007; Durbin \& Wilson, 2012; Hayden et al., 2010; Rothbart \& Bates, 2006). Laboratory measures of child temperament have several advantages, such as reducing rater bias through standardized coding procedures and the use of standardized stimuli (e.g., Gagne, Van Hulle, Aksan, Essex, \& Goldsmith, 2011). We used a combination of exploratory and confirmatory factor analytic techniques to expand the extant literature on observed temperament in preschoolers, which consists of a single study (i.e., Dyson et al., 2012). Our work therefore provides important new information on the structure of observational measures of temperament.

Our three studies cover an important developmental period about which relatively little is known with respect to structural aspects of temperament. Our hope is that our work will facilitate future research developing a unified taxonomy of child temperament/personality, which is essential toward gaining a better understanding of the pattern of covariation between traits, their developmental trajectories, and how temperament is related to mental health and other key outcomes. While there is an established literature linking childhood temperament to symptoms of psychopathology, this work has been hampered by the lack of consensus on which traits are most important to capturing children's individual differences. Developing an overarching structure of temperament/personality traits in children is essential from the perspective of integrating findings from extant research studies via common language (Shiner \& DeYoung, 2013). 
However, conceptualizing such a unified taxonomy of child temperament/personality is complicated by the process of emergence of new traits as children get older (Shiner \& DeYoung, 2013). As a more comprehensive and rigorous multimethod literature on the structure of child temperament develops, future work will benefit from a consensus on which broad traits account for important variance in temperament. Understanding the constituents of broadband traits will further facilitate work on which level of trait variance is most useful for predicting important outcomes; in other words, while both higher- lower-order traits may have predictive utility (Caspi, 2000; Clark, 2005; Laursen, Pulkkinen, \& Adams, 2002; Pesonen, Raikkonen, Keskivaara, \& Keltikangas-Jarvinen, 2003; Shiner, Masten, \& Tellegen, 2002), work on this question will prove most useful when traits are drawn from valid structures.

It is also important to acknowledge the weaknesses of the three studies, as well as highlighting directions for future work in this field. In the first two studies, we used informant reports of child temperament provided by primary caregivers who were mostly (over 90\%) mothers. It will be important for future work to examine the nature of children's temperament structure based on father-report, a source of information that has generally been underutilized in past work. Similarly, investigating the differential predictive validity of father-reported child temperament relative to mother-reported temperament is also an important direction. Since younger children cannot readily self-report on traits, we were unable to examine the structure of the CBQ using such methods and did not collect the self-report version of the TMCQ; thus, we cannot draw conclusions regarding the structure of child self-reported temperament, an important avenue for future work. The approach used in the last study differed somewhat from the first in that we did not have a sufficiently large sample size to conduct item-level analyses of observed data, in light of the fact that 540 behaviors were coded to capture child temperament across the 
Lab-TAB. Thus, our analyses of the observed data were less comprehensive than our work on parent-report.

Another limitation concerns the limited diversity of our samples; participants were predominantly Caucasian (at least $90 \%$ in each sample) and were from largely middle- and upper-class families. It will be important to extend this research to children from a broader array of backgrounds and cultures. One of the advantages of the FFM (McCrae \& Costa, 1997) is that this model of adult personality has been extensively replicated in different cultures (McCrae \& Terracciano, 2005; McCrae, Terracciano, De Fruyt, De Bolle, Gelfand, \& Costa, 2010); future work on the structure of child temperament should ultimately strive toward similar goals.

Although our studies examined children from a range of ages in early and middle childhood, we did not specifically conduct tests of the structure of temperament across time. Several studies have provided evidence for stability of child temperament over time using parent reports (e.g., Bould, Joinson, Sterne, \& Araya, 2013; Casalin, Luyten, Vliegen, \& Meurs, 2012; Guerin \& Gottfried, 1994) and observational methods (e.g., Durbin et al., 2007; Dyson, Olino, Durbin, Goldsmith, Bufferd, Miller et al., 2015). However, there has not yet been a study that examined the structural stability of child temperament over time using measurement invariance testing in the context of exploratory structural equation modeling. In particular, it is important to examine the developmental trajectory of the structure of the higher-order NA factor: do observed fear, anger, and sadness coalesce into a single factor over time or do they remain differentiated? Examining the structure of observed temperament over time is complicated by the need for developmentally sensitive behavioral tasks. In particular, tasks tapping EC in older children have not been developed. Similarly, it will be important to design new behavioral tasks that tap constructs identified as important parts of adult personality (e.g., Openness to Experience). 
Within the context of longitudinal studies, using peer-report as an informant measure of adolescent temperament may also prove useful. For example, Ready, Clark, Watson, and Westerhouse (2000) showed that peer reports provide important information on maladaptive personality traits characterized by low social desirability (e.g., entitlement) over and above selfreports in a large sample of adolescents.

\section{Conclusion}

The first two studies showed that two widely-used parent-report measures of child temperament contain a substantial number of items that do not contribute meaningfully to the assessment of child temperament. Further, higher-order analyses of these parent-reports structures did not resemble the theoretical models posited by the authors of the measures (i.e., Rothbart et al., 2001; Simonds, 2006; Simonds \& Rothbart, 2004), nor other extant models of child and adult temperament/personality (e.g., McCrae \& Costa, 1997; Shiner \& DeYoung, 2013; Tellegen, 1985). Further, key traits held to be crucial individual difference factors in children were not evident in higher-order models based on parent-report. Overall, significant revision of these measures seems necessary. The last manuscript contributes to the small literature on the structure of observed temperament in preschoolers. Considering this study alongside the only other published work on this topic (i.e., Dyson et al., 2012) indicates that PA, Dysphoria, and Impulsivity/Anger may constitute important higher-order temperament factors in early childhood, although this literature is so small that firm conclusions cannot be made at this point. Future work developing empirically based measures of parent-reported temperament is needed, as is additional work on the nature of observed temperament. Ultimately, both parentreport and observed measures of child temperament must be examined longitudinally to develop a more accurate and coherent understanding of the nature of temperament across the lifespan, as 
well as toward understanding the predictive validity of each method for important developmental outcomes. 


\section{References}

Bould, H., Joinson, C., Sterne, J., \& Araya, R. (2013). The emotionality activity sociability temperament survey: Factor analysis and temporal stability in a longitudinal cohort. Personality and Individual Differences, 54, 628-633. doi:10.1016/ j.paid.2012.11.010

Camras, L. A., Oster, H., Campos, J., Campos, R., Ujiie, T., Miyake, K., Wang, Lei, \& Meng, Z. (1998). Production of emotional facial expressions in European, American, Japanese, and Chinese infants. Developmental Psychology, 34, 616-628. doi:10.1037/00121649.34.4.616

Casalin, S., Luyten, P., Vliegen, N., \& Meurs, P. (2012). The structure and stability of temperament from infancy to toddlerhood: A one-year prospective study. Infant Behavior \& Development, 35, 94-108. doi:10.1016/j.infbeh.2011.08.004

Caspi, A. (2000). The child is the father of the man: Personality continuities from childhood to adulthood. Journal of Personality and Social Psychology, 78, 158-172.

Clark, L. A. (2005). Temperament as a unifying basis for personality and psychopathology. Journal of Abnormal Psychology, 114, 505-521. doi:10.1037/0021843X.114.4.505

Clark, L. A., \& Watson, D. (2008). Temperament: An organizing paradigm for trait psychology Guilford Press, New York, NY.

Clifford, S., Lemery-Chalfant, K., \& Goldsmith, H. H. (2015). The unique and shared genetic and environmental contributions to fear, anger, and sadness in childhood. Child Development, doi:10.1111/cdev.12394

De Pauw, S. S. W., Mervielde, I., \& Van Leeuwen, K. G. (2009). How are traits related to 
problem behavior in preschoolers? similarities and contrasts between temperament and personality. Journal of Abnormal Child Psychology,37, 309-325. doi: 10.1007/s10802008-9290-0

Digman, J. M., \& Shmelyov, A. G. (1996). The structure of temperament and personality in Russian children. Journal of Personality and Social Psychology,71, 341-351. doi:10.1037/0022-3514.71.2.341

Durbin, C. E., Hayden, E. P., Klein, D. N., \& Olino, T. M. (2007). Stability of laboratoryassessed temperamental emotionality traits from ages 3 to 7 . Emotion, 7, 388-399. doi:10.1037/1528-3542.7.2.388

Durbin, C. E., \& Wilson, S. (2012). Convergent validity of and bias in maternal reports of child emotion. Psychological Assessment, 24, 647-660. doi:10.1037/a0026607

Dyson, M. W., Olino, T. M., Durbin, C. E., Goldsmith, H. H., Bufferd, S. J., Miller, A. R., \& Klein, D. N. (2015). The structural and rank-order stability of temperament in young children based on a laboratory-observational measure. Psychological Assessment, 27, 1388-1401. doi:10.1037/pas0000104

Dyson, M.W., Olino, T.M., Durbin, C.E., Goldsmith, H.H., \& Klein, D. N. (2012). The structure of temperament in preschoolers: A two-stage factor analytic approach. Emotion, 12, 4457.

Gagne, J. R., Van Hulle, C. A., Aksan, N., Essex, M. J., \& Goldsmith, H. H. (2011). Deriving childhood temperament measures from emotion-eliciting behavioral episodes: Scale construction and initial validation. Psychological Assessment, 23, 337-353. doi:10.1037/a0021746

Goldberg, L. R. (2001). Analyses of Digman's child-personality data: Derivation of Big Five 
factor scores from each of six samples. Journal of Personality, 69, 709-743.

Goldsmith, H. H., Reilly, J., Lemery, K. S., Longley, S., \& Prescott, A. (1995).

Laboratory Temperament Assessment Battery: Preschool version. Unpublished manuscript.

Guerin, D. W., \& Gottfried, A. W. (1994). Developmental stability and change in parent reports of temperament: A ten-year longitudinal investigation from infancy through preadolescence. Merrill-Palmer Quarterly, 40, 334-355.

Hayden, E. P., Durbin, C. E., Klein, D. N., \& Olino, T. M. (2010). Maternal personality influences the relationship between maternal reports and laboratory measures of child temperament. Journal of Personality Assessment, 92, 586-593. doi:10.1080/ 00223891.2010 .513308

Hayden, E. P., Klein, D. N., \& Durbin, C. E. (2005). Parent reports and laboratory assessments of child temperament: A comparison of their associations with risk for depression and externalizing disorders. Journal of Psychopathology and Behavioral Assessment, 27, 89100. doi:10.1007/s10862-005-5383-Z

Izard, C. E., Fantauzzo, C. A., Castle, J. M., Haynes, O. M., Rayias, M. F., \& Putnam, P. H. (1995). The ontogeny and significance of infants' facial expressions in the first 9 months of life. Developmental Psychology, 31, 997-1013. doi:10.1037/0012-1649.31.6.997

John, O. P., Naumann, L. P., \& Soto, C. J. (2008). Paradigm shift to the integrative Big Five trait taxonomy: History: measurement, and conceptual issue. In O. P. John, R. W. Robins, \& L. A. Pervin (Eds). Handbook of personality: Theory and research (pp. 114-158). New York: Guilford Press.

Kagan, J. (1998). In Eisenberg N. (Ed.), Biology and the child. Hoboken, NJ, US: John 
Wiley \& Sons Inc, Hoboken, NJ.

Kochanska, G., \& Knaack, A. (2003). Effortful control as a personality characteristic of young children: Antecedents, correlates, and consequences. Journal of Personality, 71, 10871112. doi:10.1111/1467-6494.7106008

Kotelnikova, Y., Kryski, K.R., Klein, D.N., \& Hayden, E.P. (2016a; in preparation). Structure of observed temperament in preschoolers and associations with internalizing and externalizing symptoms.

Kotelnikova, Y., Mackrell, S. V. M., Jordan, P. L., \& Hayden, E. P. (2015). Longitudinal associations between reactive and regulatory temperament traits and depressive symptoms in middle childhood. Journal of Clinical Child and Adolescent Psychology, 44, 775-786. doi:10.1080/15374416.2014.893517

Kotelnikova, Y., Olino, T.M., Klein, D.N., Kryski, K.R., \& Hayden, E.P. (2016b). Higherand lower-order factor analyses of the Children's Behavior Questionnaire in early and middle childhood. Psychological Assessment, 28. 92-108. doi: 10.1037/pas0000153

Kotelnikova, Y., Olino, T.M., Klein, D.N., Mackrell, S.V.M., \& Hayden, E.P. (2016c). Higher- and lower-order factor analyses of the Temperament in Middle Childhood Questionnaire (TMCQ). Assessment. doi: 10.1177/1073191116639376

Kotelnikova, Y., Olino, T. M., Mackrell, S. V. M., Jordan, P. L., \& Hayden, E. P. (2013). Structure of observed temperament in middle childhood. Journal of Research in Personality, 47, 524-532. doi:10.1016/j.jrp.2013.04.013

Lamb, M. E., Chung, S. S., Wessels, H., Broberg, A. G., \& Hwang, C. P. (2002). Emergence and construct validation of the big five factors in early childhood: A longitudinal analysis of their ontogeny in Sweden. Child Development, 73, 1517-1524. 
Laursen, B., Pulkkinen, L., \& Adams, R. (2002). The antecedents and correlates of agreeableness in adulthood. Developmental Psychology, 38, 591-603.

McCrae, R. R., \& Costa, P. T. (1997). Personality trait structure as a human universal. American Psychologist, 52, 509-516. doi:10.1037/0003-066X.52.5.509

McCrae, R. R., \& Terracciano, A. (2005). Personality profiles of cultures: Aggregate personality traits. Journal of Personality and Social Psychology, 89, 407-425. doi:10.1037/00223514.89.3.407

McCrae, R. R., Terracciano, A., De Fruyt, F., De Bolle, M., Gelfand, M. J., \& Costa, P. T., Jr. (2010). The validity and structure of culture-level personality scores: Data from ratings of young adolescents. Journal of Personality, 78, 815-838. doi:10.1111/j.14676494.2010.00634.x

Nigg, J. T. (2006). Temperament and developmental psychopathology. Journal of Child Psychology and Psychiatry, 47, 395-422. doi:10.1111/j.1469-7610.2006.01612.x

Pauli-Pott, U., Mertesacker, B., Bade, U., Haverkock, A., \& Beckmann, D. (2003). Parental perceptions and infant temperament development. Infant Behavior \& Development, 26, 27-48. doi:10.1016/S0163-6383(02)00167-4

Pesonen, A.-K., Raikkonen, K., Keskivaara, P., \& Keltikangas-Jarvinen, L. (2003). Difficult temperament in childhood and adulthood: Continuity from maternal perceptions to selfratings over 17 years. Personality and Individual Differences, 34, 19-31.

Pytlik Zillig, L. M., Hemenover, S. H., \& Dienstbier, R. A. (2002). What do we assess when we assess a big 5 trait? A content analysis of the affective, behavioral and cognitive processes represented in the big 5 personality inventories. Personality and Social Psychology Bulletin, 28, 847-858. doi:10.1177/0146167202289013 
Rohlf, H. L., \& Krahé, B. (2015). Assessing anger regulation in middle childhood: Development and validation of a behavioral observation measure. Frontiers in Psychology, 6.

Rothbart, M. K., Ahadi, S. A., Hershey, K. L., \& Fisher, P. (2001). Investigations of temperament at three to seven years: The children's behavior questionnaire. Child Development, 72, 1394-1408. doi:10.1111/1467-8624.00355

Rothbart, M.K. \& Bates, J.E. (2006). In Handbook of child psychology: Vol. 3 Social, emotional, and personality development (6th ed.), by Rothbart, Mary K., Bates, John E., 99-166. Hoboken, NJ, US: John Wiley \& Sons Inc.

Ready, R. E., Clark, L. A., Watson, D., \& Westerhouse, K. (2000). Self- and peer-related personality: Agreement, trait ratability, and the "self-based heuristic". Journal of Research in Personality, 34, 208-224.

Shiner, R. L., \& DeYoung, C. G. (2013). The structure of temperament and personality traits: A developmental perspective. The oxford handbook of developmental psychology, vol. 2: Self and other. (pp. 113-141) Oxford University Press, New York, NY.

Shiner, R. L., Masten, A. S., \& Tellegen, A. (2002). A developmental perspective on personality in emerging adulthood: Childhood antecedents and concurrent adaptation. Journal of Personality and Social Psychology, 83, 1165-1177.

Simonds, J. (2006). The role of reward sensitivity and response: Execution in childhood extraversion. Unpublished doctoral dissertation, University of Oregon.

Simonds, J. \& Rothbart, M. K. (2004). The Temperament in Middle Childhood Questionnaire (TMCQ): A computerized self-report measure of temperament for ages 710. Poster session presented at the Occasional Temperament Conference, Athens, GA.

Tellegen, A. (1985). Structure of mood and personality and their relevance to assessing anxiety, 
with an emphasis on self-report. In A. H. Tuma \& J. D. Maser (Eds.), Anxiety and the anxiety disorders (pp. 681-706). Hillsdale, NJ: Erlbaum.

Watson, D., \& Clark, L. A. (1993). In Wegner D. M., Pennebaker J. W. (Eds.), Behavioral disinhibition versus constraint: A dispositional perspective. Englewood Cliffs, NJ, US: Prentice-Hall, Inc, Englewood Cliffs, NJ.

Wilt, J., \& Revelle, W. (2009). Extraversion. Handbook of individual differences in social behavior. (pp. 27-45) Guilford Press, New York, NY. 


\title{
Appendix A: Ethics Approval for the Data Used in "Higher- and Lower-Order Factor Analyses of the Children's Behavior Questionnaire in Early and Middle Childhood" and “The Structure of Observed Temperament in Preschoolers" ON Sample
}

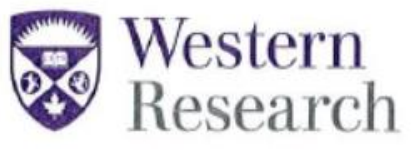

\author{
Western University Health Science Research Ethics Board \\ NMREB Annual Continuing Ethics Approval Notice
}

Research Ethics

Date: May 31, 2015

Principal Investigator: Prof. Elizabeth Hayden

Department \& Institution: Social SciencelPsychology,Western University

NMREB File Number: 5246

Study Title: Gene-Environment Interplay and the Development of Child Temperament - 15121S

Sponsor: Canadian Institutes of Health Research

NMREB Renewal Due Date \& NMREB Expiry Date:

Renewal Due -2016/05/31

Expiry Date -2016/06/11

The Western University Non-Medical Research Ethics Board (NMREB) has reviewed the Continuing Ethics Review (CER) form and is re-issuing approval for the above noted study.

The Western University NMREB operates in compliance with the Tri-Council Policy Statement Ethical Conduct for Research Involving Humans (TCPS2), Part 4 of the Natural Health Product Regulations, the Ontario Freedom of Information and Protection of Privacy Act (FIPPA, 1990), the Ontario Personal Health Information Protection Act (PHIPA, 2004), and the applicable laws and regulations of Ontario.

Members of the NMREB who are named as Investigators in research studies do not participate in discussions related to, nor vote on such studies when they are presented to the REB.

The NMREB is registered with the U.S. Department of Health \& Human Services under the IRB registration number IRB 00000941. 


\section{Appendix B: Ethics Approval for the Data Used in "Higher- and Lower-Order Factor}

\section{Analyses of the Temperament in Middle Childhood Questionnaire" ON sample}

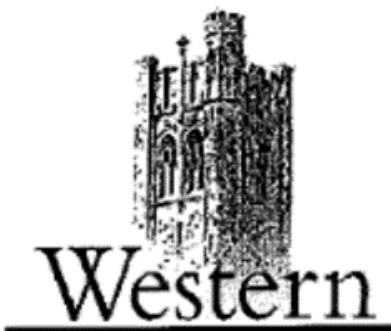

Principal Investigator: Dr. E.P. Hayden

Review Number: 12279S

Review Date: November 05, 2009

Protocol Title: Child Temperament and Individual Differences in Information Processing

Department and Institution: Psychology, University of Western Ontario

Sponsor:

Ethics Approval Date: November 11, 2009

Expiry Date: June 30, 2010

Documents Reviewed and Approved: Revised study methodology. Letter of Information and Consent.

This is to notify you that The University of Western Ontario Research Ethics Board for Non-Medical Research Involving Human Subjects (NMREB) which is organized and operates according to the Tri-Council Policy Statement: Ethical Conduct of Research Involving Humans and the applicable laws and regulations of Ontario has granted approval to the above referenced revision(s) or amendment(s) on the appreval date noted above.

This approval shall remain valid until the expiry date noted above assuming timely and acceptable responses to the NMREB's periodic requests for surveillance and monitoring information. If you require an updated approval notice prior to that time you must request it using the UWO Updated Approval Request Form.

During the course of the research, no deviations from, or changes to, the study or consent form may be initiated without prior written approval from the NMREB except when necessary to eliminate immediate hazards to the subject or when the change(s) involve only logistical or administrative aspects of the study (e.g. change of monitor, telephone number). Expedited revicw of minor change(s) in ongoing studies will be considered. Subjects must receive a copy of the signed information/consent documentation.

Investigators must promptly also report to the NMREB:

a) changes increasing the risk to the participant(s) and/or affecting significantly the conduct of the study;

b) all adverse and unexpected experiences or events that are both serious and unexpected;

c) new information that may adversely affoct the safety of the subjects or the conduct of the study.

If these changes/adverse events require a change to the information/consent documentation, and/or recruitment advertisement, the newly revised information/consent documentation, and/or advertisement, must be submitted to this office for approval.

Members of the NMREB who are named as investigators in research studies, or declare a conflict of interest, do not participate in discussion related to, nor vote on, such studies when they are presented to the NMREB. 
Appendix C: Ethics Approval for the Data Used in "Higher- and Lower-Order Factor

Analyses of the Children's Behavior Questionnaire in Early and Middle Childhood", "Higher- and Lower-Order Factor Analyses of the Temperament in Middle Childhood

Questionnaire", and "The Structure of Observed Temperament in Preschoolers" NY

Sample

$\star \|$ Stony Brook Research

Stony Brook University Institutional Review Board (IRB)

DATE:

TO:

FROM:

SUBMISSION TYPE:

STUDY TITLE:

CORIHS\#:

ACTION:

SUBMISSION APPROVAL DATE:

PROJECT EXPIRATION DATE:

REVIEW TYPE:

EXPEDITED REVIEW CATEGORY:
May 19, 2015

Daniel Klein, PhD

Stony Brook University IRB (CORIHS A)

Continuing Review/Progress Report

[88933-34] Observations of Active and Inactive Children (Temperamental Low PE in Preschoolers and Depression Risk)

2007-2917-R8

APPROVED

May 19, 2015

May 18, 2016

Expedited Review

Thank you for your submission of Continuing Review/Progress Report materials for this research study. Stony Brook University IRB (CORIHS A) (FWA \#00000125) has APPROVED your submission.

All research must be conducted in accordance with this approved submission. Any modifications to the study as approved must be reviewed and approved by CORIHS prior to initiation.

If this activity has components that require approval from additional compliance committees (e.g., IACUC, IRB, IBC, SCRO, COI) it is your responsibility to not commence with the study until these approvals have been secured as well.

Please note:

- Consent forms signed by subjects in this study must be kept by the investigator for 6 (six) years from study termination, or indefinitely (if so indicated in the consent form).

- Inclusion of minors in this study is acceptable in accordance with 45 CFR 46404

- Parental permission and minor assent is obtained in accordance with 45 CFR 46.408. Permission from one parent is acceptable. Minor assent is also obtained in accordance with SBU Assent Policy, Category 1.

- Approval includes amendment adding 2 saliva samples per Amendment Narrative submitted. Please update protocol with this amendment and submit with next submission package. Please note that amendments should be submitted with amendment cover sheet, available in IRBNet.

You are reminded that you must apply for, undergo review, and be granted continued approval for this study before May 18, 2016 in order to be able to conduct your study in an uninterrupted manner. If you do not receive approval before this date, you must cease and desist all research involving human subjects, their tissue and their data until such time as approval is granted. 


\section{YULIYA KOTELNIKOVA}

\section{CURRICULUM VITAE}

\section{Personal Information}

Address:

Email:

Telephone:

\section{$\underline{\text { Education }}$}

Sept.2016 - Aug.2017 Psychology Resident

Child and Adolescent Clinical Psychology Track

IWK Health Centre

Halifax, NS, Canada

Sept. 2012 - Present

Ph.D. Candidate, Clinical Psychology

Western University

London, ON, Canada

Sept. 2010 - July, $2012 \quad$ Master of Science, Clinical Psychology

Western University

London, ON, Canada

Sept. 2005 - June 2009 Honours Bachelor of Science (Psychology Research Specialist)

(High Distinction), University of Toronto

Toronto, ON, Canada

Aug. 2000 - Dec.2003 International Business Diploma

(High Honours), Centennial College

Toronto, ON, Canada

\section{$\underline{\text { Research Scholarships \& Fellowships }}$}

2011-2015

2012-2016

2011-2012
Children's Health Research Institute (CHRI) Graduate Fellowship (sought and awarded each year)

Social Sciences and Humanities Research Council (SSRHC)

Doctoral Fellowship

Ontario Graduate Scholarship 
2010-2011

2010-2011

2010-present

$\underline{\text { Academic Awards }}$

2013

2009

2009

2009

2006, 2009

2002, 2003

\section{$\underline{\text { Travel Awards }}$}

Joseph-Armand Bombardier Master's Research Scholarship awarded by the Social Sciences and Humanities Research Council

Ontario Graduate Scholarship

Western Graduate Research Scholarship awarded by Western University

Marilyn (Pack) McClelland Award Awarded to a full-time graduate student in Developmental of Clinical Psychology (Western University) based on academic achievement, research productivity, and quality of research publications

Certificate of Academic Excellence Awarded by the Canadian Psychological Association to an honours thesis student on the recommendation of the University of Toronto, Department of Psychology

The Erik Jackman Scholarship in Psychology Awarded to a Trinity college (University of Toronto) student with the highest CGPA in psychology research specialist program

The Trinity College Scholar (University of Toronto) Awarded based on academic merit

University of Toronto Dean's List Awarded to top $10 \%$ of University of Toronto students with CGPA of 3.5/4 or higher

Centennial College: International Students' Excellence Award Awarded to an international student with the highest CGPA

Western University Graduate Studies Travel Bursary

Behavior Genetics Association (BGA) Travel Bursary

2009

Quantitative Training for Underrepresented Groups (QTUG)

Stipend 


\section{$\underline{\text { Publications }}$}

Kotelnikova, Y., LeMoult, J., Mackrell, S.V.M., Sheikh, H.I., Singh, S. M., Joormann, J., Gotlib, H.I, \& Hayden, E.P. (in press). The serotonin transporter promoter variant, stress, and attentional biases in middle childhood. Personality and Individual Differences. doi: 10.1016/j.paid.2016.06.004

Mackrell, S. V.M., Kotelnikova, Y., Jordan, P., \& Hayden, E.P. (in press). The role of pubertal development in emerging depression risk in middle childhood. Personality and Individual Differences. doi:10.1016/j.paid.2016.03.035

Kotelnikova, Y., Olino, T.M., Klein, D.N., Mackrell, S.V.M., \& Hayden, E.P. (2016). Higher- and lower-order factor analyses of the Temperament in Middle Childhood Questionnaire (TMCQ). Assessment. doi: 10.1177/1073191116639376

Kotelnikova, Y., Olino, T.M., Klein, D.N., Kryski, K.R., \& Hayden, E.P. (2016). Higherand lower-order factor analyses of the Children's Behavior Questionnaire in early and middle childhood. Psychological Assessment, 28. 92-108. doi: 10.1037/pas0000153

Kotelnikova Y., Vernon, P.A., Clark, L.A., \& Hayden, E.P. (2015). Development and validation of the Schedule for Nonadaptive and Adaptive Personality - Brief SelfDescription Rating Form (SNAP - BSRF). Assessment, 22, 3-16. doi: 1073191114534959

Kotelnikova, Y., Mackrell, S. V. M., Jordan, P. L., \& Hayden, E. P. (2015). Longitudinal associations between reactive and regulatory temperament traits and depressive symptoms in middle childhood. Journal of Clinical Child and Adolescent Psychology, 44, 775-786. doi:10.1080/15374416.2014.893517

Mackrell, S.V.M., Sheikh, H. I., Kotelnikova, Y., Kryski, K.R., Jordan, P.L., Singh, S.M., \& Hayden, E.P. (2014). Child temperament and parent depression predict cortisol reactivity to stress in middle childhood. Journal of Abnormal Psychology. 123, 106-116. doi:10.1037/a0035612

Kotelnikova, Y., Olino, T.M., Mackrell, S. V.M., Jordan, P. L., \& Hayden, E.P. (2013). Structure of observed temperament in middle childhood. Journal of Research in Personality, 47, 524-532, doi: 10.1016/j.jrp.2013.04.013

Kotelnikova, Y. (2011). Review of personality science: Three approaches and their applications to the causes and treatment of depression. Personality and Individual Differences, 51(7), 877-878. doi:10.1016/j.paid.2011.07.016

Kotelnikova, Y. \& Tackett, J.L. (2009). Personality and cultural correlates of childhood psychopathology. Journal of Undergraduate Life Sciences, 3, 26-30. 


\section{$\underline{\text { Manuscripts in Submission }}$}

Sheikh, H.I., Kryski, K.R., Kotelnikova, Y., Hayden, E.P., \& Singh, S.M. (in submission). Catechol-O-Methyltransferase gene (val158met) polymorphisms and anxiety symptoms in early childhood: The role of hypothalamus-pituitary-adrenal axis reactivity and stress. Genes, Brain, \& Behavior.

\section{$\underline{\text { Manuscripts in Preparation }}$}

Kotelnikova Y., Vernon, P.A., Clark, L.A., \& Hayden, E.P. (in preparation). Development and validation of the Schedule for Nonadaptive and Adaptive Personality - Brief OtherDescription Rating Form (SNAP - BORF).

Kotelnikova, Y., Kryski, K.R., Olino, T.M., Klein, D.N., \& Hayden, E.P. (in preparation). Structure of observed temperament in preschoolers and associations with internalizing and externalizing symptoms.

Kotelnikova, Y. \& Hayden, E.P. (in preparation). Child temperament. In Encyclopedia of Personality and Individual Differences. New York, NY: Springer.

\section{Non-peer-reviewed Publications:}

Kotelnikova, Y. \& Ragsdale, K. (2010). Interview with Dr. Thomas Oltmanns, 2009 SRP president, Society for Research in Psychopathology Annual Newsletter.

\section{$\underline{\text { Presentations }}$}

Kotelnikova, Y., Sheikh, H.I., Vandermeer, M.R.J., Kryski, K.R., Singh, S.M., Olino, T.M., \& Hayden, E.P. (2016). Predictors of Latent Cortisol Trajectory Classes in the Context of Stress. Poster presented at the Association for Psychological Science $28^{\text {th }}$ Annual Convention, Chicago, IL, USA.

Vandermeer, M.R.J., Kotelnikova, Y., Kryski, K.R., \& Hayden, E.P. (2016). Relationship Quality Moderates Mother-Father Personality Agreement. Poster presented at the Association for Psychological Science $28^{\text {th }}$ Annual Convention, Chicago, IL, USA.

Kotelnikova, Y., Kryski, K.R., \& Hayden, E.P. (2015). Structure of Observed Temperament in Preschoolers and Associations with Internalizing and Externalizing Symptoms. Poster presented at the Society for Research in Psychopathology Annual Meeting, New Orleans, Louisiana, USA.

Kotelnikova, Y., Olino, T.M., Klein, D.N., \& Hayden, E.P. (2015). Nature and Structure of Temperament in Early and Middle Childhood: A Multi-Method Approach. Talk presented at the International Society for the Study of Individual Differences Conference, London, ON, Canada.

Kotelnikova, Y., Mackrell, S.V.M., Sheikh, H.I., Singh, S.M., \& Hayden, E.P. (2014). The 
Serotonin Transporter Gene and Stress Interact to Predict Children's Attentional Biases for Positive Stimuli. Poster presented at the Society for Research in Psychopathology Annual Meeting, Evanston, Illinois, USA.

Mackrell, S.V.M., Kotelnikova, Y., Jordan, P., \& Hayden, E.P. (2014). Pubertal Status Moderates Associations between Depression Risks and Children's Depressive Symptoms. Poster presented at the Society for Research in Psychopathology Annual Meeting, Evanston, Illinois, USA.

Kotelnikova, Y., Vernon, P.A., Clark, L.A., \& Hayden, E.P. (2013, September). Initial Validation of the Schedule for Nonadaptive and Adaptive Personality Brief Self-Rating Form (SNAP-BSRF). Poster presented at the Society for Research in Psychopathology Annual Meeting, Oakland, California, USA.

Kotelnikova, Y., Olino, T.M., Kryski, K.R., Smith, H.J., Hayden, E.P., \& Klein, D.N. (2013, April). Revising the Children's Behavior Questionnaire (CBQ): Item-response and factor analyses. Poster presented at the Society for Research in Child Development Biennial Meeting, Seattle, WA, USA.

Mackrell, S.V.M., Sheikh, H. I., Kotelnikova, Y., Jordan, P.L., Singh, S.M., \& Hayden, E.P. (2013, April). Child temperament and parental history of internalizing disorders: Associations with HPA axis reactivity in middle childhood. Poster presented the Society for Research in Child Development Biennial Meeting, Seattle, WA, USA.

Kotelnikova, Y., Mackrell, S.V.M., Jordan, P.L., \& Hayden, E.P. (2012, October). Effortful control and cognitive vulnerability to depression in middle childhood: A multi-method, longitudinal study. Poster presented at the Society for Research in Psychopathology Annual Meeting, Ann Arbor, Michigan, USA.

Kotelnikova, Y., Mackrell, S.V.M., Veselka, L., Aitken Schermer, J., Hayden, E.P., \& Vernon, P.A. (2011, June). Non-adaptive personality traits and the Dark Triad: A behavior genetic investigation into the relationship between the two domains. Poster presented at the Behavior Genetics Association's 41st Annual Meeting, Newport, RI, USA.

Mackrell, S.V.M., Kotelnikova, Y, Veselka, L., Aitken Schermer, J., \& Vernon, P.A. (2011, June). A behavioral genetic study of sub-clinical personality disorders and trait emotional intelligence. Poster presented at the Behavior Genetics Association's 41st Annual Meeting, Newport, RI, USA.

Kotelnikova, Y., Veselka, L., Aitken Schermer, J., Hayden, E.P., \& Vernon, P.A. (2011, June). Non-adaptive personality traits and the Dark Triad: An investigation into the relationship between the two domains. Poster presented at the Canadian Psychological Association 72nd Annual Convention, Toronto, ON, Canada.

Kotelnikova, Y., Mackrell, S. V.M., Jordan, P., \& Hayden, E.P. (2011, May). Temperament 
traits as predictors of change in internalizing problems in middle childhood. Poster presented at the Association for Psychological Science $23^{\text {rd }}$ Annual Convention, Washington DC, USA.

Kotelnikova, Y. \& Tackett, J.L. (2010, October). Values as predictors of psychopathology in a multicultural Canadian sample. Poster presented at the Society for Research in Psychopathology Annual Meeting, Seattle, WA, USA.

Kotelnikova, Y. \& Tackett, J.L. (2009, August). Personality and the 10 universal values: An integrated hierarchical approach. Poster presented at the Quantitative Training for Underrepresented Groups, Toronto, ON, Canada.

Kotelnikova, Y. \& Tackett, J.L. (2009, July). Integrating personality and values for a better understanding of cross-cultural differences. Poster presented at the Annual Conference for the Association of Research in Personality, Evanston, IL, USA.

Tackett, J.L. \& Kotelnikova, Y. (2009, July). Assessing personality in middle childhood: Evidence from a multi-method, multi-informant, multi-trait study. Talk presented at the Annual Conference for the Association of Research in Personality, Evanston, IL, USA.

Kotelnikova, Y. (2009, May). Estimation of food intake in restrained and unrestrained eaters. Talk presented at the 39th Annual Ontario Psychology Undergraduate Thesis Conference, Hamilton, ON, Canada.

Kotelnikova, Y. (2009, April). Estimation of food intake in restrained andunrestrained eaters. Poster presented at the University of Toronto Undergraduate Thesis Poster Day, Toronto, ON, Canada.

Kotelnikova Y. \& Tackett, J.L. (2008, October). Personality correlates of cross-cultural differences in childhood psychopathology. Poster presented at the Mechanisms of Change in Developmental Psychopathology Symposium, Toronto, ON, Canada.

Kotelnikova Y. \& Tackett, J.L. (2008, July). Personality correlates of cross-cultural differences in childhood psychopathology. Poster presented at the 14th European Conference on Personality, Tartu, Estonia.

Kotelnikova Y. (2008, May). Personality correlates of cross-cultural differences in childhood psychopathology. Talk presented at the 38th Annual Ontario Psychology Undergraduate Thesis Conference, St. Catharines, ON, Canada.

\section{Memberships in Professional Societies}

2010 - present Society for a Science of Clinical Psychology (Student Member)

2010 - present Society for Research in Psychopathology (Associate Member)

2014 - present Canadian Psychological Association (Student Member) 


\section{Professional Service}

\section{Ad-hoc Reviewer:}

Psychological Assessment

Personality and Individual Differences

Journal of Abnormal Psychology

Journal of Abnormal Child Psychology

Behavior Therapy

Child Development

Canadian Psychology

Canadian Journal of Behavioural Science

\section{Community Talks:}

Kotelnikova, Y. \& Jones, E. (2013, February). Suicide Risk Awareness. Talk presented at the grade 9 Assembly, H.B. Beal Secondary School, London, ON, Canada.

Kotelnikova, Y. (2012, February). Temperament and depression. Talk presented as a part of the 2013 Finding Your Way Library Series, London, ON, Canada.

Kotelnikova, Y. (2012, November \& 2013, March). Introduction to distress tolerance. Talk presented as a part of the Laura Evans Lecture Series at the Student Development Center, Western University, London, ON, Canada.

\section{Committee Memberships:}

$2013-2015$

$2013-2014$

$2012-2013$

\section{Teaching Experience}

Sept.2015- May, 2015

Oct. 2014
Clinical Student Advisory Committee; Western University

Accreditation and Professional Issues Committee; Western University

Advocacy through Action: Bringing Psychology to Our Community (Pamphlet Committee); Western University
Psychology Independent Study Supervision

Student: Ashley Amicarelli

Topic: Observational Measures of Effortful Control

Western University, London, ON

Guest Lecture: Substance Use Disorders

Abnormal Child Psychology (PSY2320)

Course Instructor: Dr. E.P. Hayden

Western University, London, ON 
Sept.2014 - Dec.2014

Sept.2013- May, 2014

Mar. 2013

2012-2013

Feb. 2012

2011

2010

\section{Quantitative Training}

Graduate Teaching Assistant

Abnormal Child Psychology (PSY2320)

Course Instructor: Dr. E.P. Hayden

Western University, London, ON

Psychology Honors Thesis Supervision

Student: Talia Kish

Topic: Cognitive and temperamental vulnerability to depression in middle childhood

Western University, London, ON

Guest Lecture: Anxiety Disorders

Abnormal Child Psychology (PSY2320)

Course Instructor: Dr. E.P. Hayden

Western University, London, ON

Graduate Teaching Assistant

Abnormal Child Psychology (PSY 2320)

Course Instructor: Dr. E. P. Hayden

Western University, London, ON

Guest Lecture: Personalities of Famous People

Special Topics in Clinical Psychology: Personality and

Developmental Psychopathology (PSY 4390)

Course Instructor: Dr. E. P. Hayden

Western University, London, ON

Graduate Teaching Assistant

The Psychology of Prosocial and Antisocial Behavior (PSY 3720)

Course Instructor: Dr. D. Hazlewood

Western University, London, ON

Graduate Teaching Assistant

Introduction to Social Psychology (PSY 2720, online course)

Course Instructor: C. Wilbur (Ph.D. candidate)

Western University, London, ON

Graduate Teaching Assistant

Introduction to Psychology (PSY 1000)

Course Instructor: Dr. L. Fazakas-DeHoog

Western University, London, ON 
Graduate course taken at Western University, London, ON

2012

2012

2011

2009

\section{Clinical Training}

Sept. 2015 - May 2016

Mar.2015 - Aug. 2015

Mar. 2014-Aug. 2015

Sept.2014 - May 2015
International Workshop on Statistical Genetics and Methodology of Twin and Family Studies: The Introductory Course Boulder, Colorado, USA

Structural Equation Modeling Graduate course taken at Western University, London, ON

Introduction to Structural Equation Modeling Workshop offered by a guest instructor Dr. T. M. Olino at Western University, London, ON

Quantitative Training for Underrepresented Groups (Toronto, ON)

Intervention/Assessment Practicum

Vanier Children's Services, London, ON

Child and Family Community Services

Supervisor: Dr. Jeff Carter

Population: children, adolescents, and families

Presenting issues: family conflict, aggressive and defiant behavior, mood and anxiety disorders

Intervention Practicum

Children's Hospital: London Health Sciences Centre, London, ON

Outpatient Mood and Anxiety Service

Supervisor: Dr. Julie Eichstedt

Population: children and adolescents

Presenting issues: OCD, mood and anxiety disorders

Assessment/Intervention Practicum

Private Practice, London, ON

Supervisor: Dr. Darlene Elliott-Faust

Population: children \& adolescents

Type of assessments: psychoeducational, diagnostic clinical

Intervention/Assessment Practicum

Child and Parent Resource Institute, London, ON

Inpatient Tertiary Care Service

Supervisor: Dr. Jeff St.Pierre

Population: children $\&$ adolescents in tertiary care

Type of assessments: cognitive, diagnostic clinical, selective mutism

Presenting issues: gender identity, mood and anxiety disorders, psychosis, OCD, emerging personality disorders, 
May 2014-Sept.2014

Sept.2013 - present

May 2013 - Sept.2013

Sept.2012 - May 2013

Jan. 2012 - Jun. 2012

Jan. 2012 - Apr.2012
Assessment Practicum

Victoria Hospital: Neuropsychology, London, ON

Inpatient and Outpatient Neuropsychology Service

Supervisor: Dr. Andrea Lazosky

Population: adults \& adolescents

Type of assessments: neuropsychological

Intervention/Assessment Practicum

Thames Valley District School Board, London, ON

Supervisor: Dr. Barb Richardson

Population: adolescents

Presenting issues: internalizing and externalizing disorders, interpersonal issues

Type of assessments: psychoeducational, diagnostic clinical, risk of violence

Intervention Practicum

Regional Mental Health Care, St. Joseph's Hospital, London, ON Outpatient Mood and Anxiety Tertiary Care Service

Supervisor: Dr. Farida Spencer

Population: adults

Presenting issues: mood and anxiety disorders; personality disorders

Intervention Practicum

Student Development Center, Western University, London, ON

Supervisor: Dr. Kathryn Dance

Population: adolescents, young adults

Presenting issues: mood and anxiety disorders, stress coping, interpersonal issues, personality disorders

Child Assessment Practicum

Centre for Children and Families in the Justice System of the London Family Court Clinic, London, ON

Supervisor: Dr. Karen Bax

Population: children and adolescents

Type of assessments: trauma informed, cognitive, diagnostic clinical

Adult Assessment Practicum

University Hospital, London, ON

Outpatient Neuropsychology Service

Supervisor: Dr. Michael Harnadek

Population: older adults

Type of assessments: neuropsychological 